\title{
Poster Session
}

(C) Indian Society of Gastroenterology 2010

\section{Esophagus}

\section{E1}

\section{Dysphagia in elderly male: $A$ case report}

A Chezhian, D Sasi Anand, S Arul Prakash, S Chitra, $G$ Ram Kumar, $R$ Bala Murali, $K$ Muthu Kumaran, T Pugazhendhi, S Jeevan Kumar.

Department of Digestive Health and Diseases, Government Kilpauk Medical College, Chennai 600 010, India

A 62-year-old male presented to our department with intermittent non progressive dysphagia for the past 2 years. The patient reported an increasing frequency and duration of the symptoms which were independent from food intake, medication. There was no history of recurrent heart burn, nasal regurgitation, nocturnal cough or stale food vomiting. On examination, his vital signs were stable. Cardiac evaluation was normal. Barium swallow showed multiple diverticulae in lower end of esophagus. Upper GI scopy confirmed the finding. Esophageal manometry was performed which revealed a characteristic pattern of nutcracker esophagus (esophageal motility disorder). Diagnosis is epiphrenic diverticulae of lower end of esophagus secondary to motility disorder. Discussion Esophageal diverticula may be either of traction type, being composed of all layers of the esophageal wall including muscle coat, or pulsion type where the diverticular sac is composed of mucosa alone. The former are often associated with hilar lymphadenopathy secondary to tuberculosis, while pulsion diverticula are produced by high intraluminal pressure often as a result of some esophageal motility disorder. Esophageal diverticula may involve the proximal esophagus (Zenker's) or distal esophagus (epiphrenic). They occur in the terminal $10-15 \mathrm{~cm}$ of the esophagus, usually in middle-aged and elderly male. Distal esophageal pulsion diverticula are often secondary to motor abnormalities of the esophagus or, more rarely, distal mechan- ical obstruction. In symptomatic patients $25 \%$ have an associated achalasia, and only $10 \%$ have diffuse esophageal spasm; $3 \%$ had a hypertensive lower esophageal sphincter and $20 \%$ had non-specific motor abnormalities of the esophagus.we report this case for its rarity and its diagnostic imaging features.

\section{E2}

Role of radiofrequency ablation (HALO360 ablation system) in Barrett's esophagus

Nitesh Pratap, D Nageshwar Reddy, D Santosh, Mohan Ramchandani, Rupa Banerjee, Sandeep Lakhtakia, Rajesh Gupta, Manu Tandan, P N Rao, G V Rao.

Asian Institute of Gastroenterology, Hyderabad 500 082, India

Background: The presence of Barrett's esophagus (BE) increases the risk of esophageal cancer. Total regression of $\mathrm{BE}$ is uncommon with medication or laparoscopic fundoplication. Endoscopic techniques to obliterate BE have varied results. We present the data of three patients with biopsy-proven BE who underwent balloon-based catheter radiofrequency ablation (RFA) with the HALO360 ablation system.

Methods: The patients underwent upper GI endoscopy and narrow band imaging before ablation to document presence of $\mathrm{BE}$ and again at 8 weeks after the RFA to assess the presence of any residual BE. An esophageal manometry was done to rule out motility disorder.

Results: Mean patient age was 52.33 years; all were men. They had presented with heartburn and reflux symptoms. The average length of the Barrett segment treated was $4.0 \pm 2 \mathrm{~cm}$. Two patients had visible mucosal abnormality with high grade dysplasia for which they underwent endoscopic mucosal resection 8 weeks prior to RFA. One patient had low-grade dysplasia. None of patients had esophageal carcinoma. There was no procedural morbidity and no postprocedure dysphagia or stricture at follow-up. BE was completely replaced with squamous epithelium in all the subjects. 
Conclusions: RFA for BE is safe and achieves $100 \%$ replacement of intestinal metaplasia in patients.

\section{E3}

Pneumatic dilation for achalasia cardia: Reduction in lower esophageal sphincter pressure in assessing response and factors associated with recurrence during long-term follow up

Uday C Ghoshal, Murali Rangan, Asha Misra. Department of Gastroenterology, Sanjay Gandhi Postgraduate Institute of Medical Sciences, Raebareli Road, Lucknow 226 104, India

Background: Data on utility of post-pneumatic dilation (PD) lower esophageal sphincter (LES) pressure measurement in evaluating short and long-term efficacy of dilation, which tears the non-relaxing LES in achalasia, are scanty.

Methods: Post-PD LES pressure was measured in $72 / 98$ patients with achalasia. The best cut-off pressure classifying responders and non-responders was determined by ROC curve. Factors associated with non-response and recurrence was evaluated. Results: Of 98 patients $(41.1+13.3-y, 58$ male), 75 improved, 3 had perforation requiring surgery, and $20 \mathrm{did}$ not respond to first PD session. Of 18/20 patients undergoing second PD, 11 improved and 6 non-responders needed myotomy. 37/58 (71\%) male and $17 / 40(42.5 \%)$ female had first PD with $35-\mathrm{mm}$ balloon $(p=0.03)$. Age and gender of patients did not influence outcome. LES pressure was lower in responder or those having recurrence than non-responder [17- $\mathrm{mmHg}(6.4-75)$, 11-mmHg (4.6-31) vs. 25-mmHg (13-55)]. On ROC curve, $22.5-\mathrm{mmHg}$ was the best cut-off value differentiating responders and non-responders (AUC 0.73). Of the 86 patients responding to $\mathrm{PD}, 20$ had recurrence. Patients who responded to one session of PD or had LES pressure below $10-\mathrm{mmHg}$ after procedure recurred less often, though there was no relationship with age and gender.

Conclusion: Post-PD LES pressure measurement is useful to assess treatment response. Patient responding to first session and those with post-PD LES pressure $<10-\mathrm{mmHg}$ tended to recur less. Age and gender did not influence outcome, which might be related to preferential use of $35-\mathrm{mm}$ balloon, particularly for male patients, during the first session.

\section{E4}

\section{Intralesional mitomycin $\mathrm{C}$ injection in the treatment of corrosive esophageal strictures}

Amol Samarth, C Vashishtha, Prabha Sawant, Jignesh Patel, A Agasti, M Nasax.

Department of Gastroenterology, L T M M C and L T M G H, Sion, Mumbai 400 022, India
Introduction: Mitomycin $\mathrm{C}$ is an antiproliferative agent that inhibits fibbroblast proliferation. Successful use of locally applied mitomycin $\mathrm{C}$ has been reported after esophageal stricture dilatation in children, however it was applied using a rigid endoscope. Another method described uses retrograde esophageal stricture dilatation for mitomycin $\mathrm{C}$ application. We propose a simplified method of intralesional application of mitomycin $\mathrm{C}$ in the treatment of corrosive esophageal strictures. Methods: Consecutive consenting patients with corrosive esophageal strictures who were on "on demand" protocol for esophageal dilatations from July 2009 to March 2010 were included in the study. Each underwent esophageal stricture dilatation using American endoscopic dilators. After this $0.25 \mathrm{~mL}$ each of diluted mitomycin $\mathrm{C}(0.4 \mathrm{mg} / \mathrm{mL})$ was injected at the site of stricture in four quadrants using sclerotherapy needle. Patients were observed post-procedure for $3 \mathrm{~h}$ for any adverse events. Patients were followed up for minimum of 3 months. Interval between dilatation sessions before and after mitomycin $\mathrm{C}$ was compared on follow-up. Results: Nine patients were included in the study. Mean age was $28.44 \pm 4.71$ years. There were 6 males and 3 female patients (M:F 2:1). Before mitomycin $\mathrm{C}$ application patients underwent dilations every 3-6 weeks. After single application of mitomycin $\mathrm{C}$ this duration increased to 6-11 weeks. Two patients required further two applications of mitomycin C. $6(66.6 \%)$ patients experienced post procedure chest pain. No other local or systemic side-effects were seen. Intralesional Mitomycin $\mathrm{C}$ injection resulted in significant reduction in the frequency of "on demand" esophageal dilatations $(p<0.05)$. Conclusion: Intralesional Mitomycin $\mathrm{C}$ offers a novel method in management of corrosive esophageal stricture.

\section{E5}

\section{Variant achalasia-Case report}

K Kalyan, S Rajanikanth, Shanthi Vijayaraghavan, Shanmuganathan.

Sri Ramachandra Medical College

A 58 year old male patient presented with insidious onset non progressive difficulty in swallowing for both solids and liquids for 4 months. There was no history of chest pain, heart burn, vomiting, or loss of appetite. History of weight loss is present,lost $4 \mathrm{~kg}$ in 4 months. There was no significant past history. Physical examination showed no significant abnormality. Routine blood investigations were within normal limits. Chest X Ray showed no significant abnormality. Barium Swallow showed grossly dilated esophagus with pointed bird's beak appearance in the distal esophagus. CT Chest showed a dilated esophagus. OGD showed a grossly dilated esophagus with lot of food and fluid residue. Esophageal manometry showed simultaneous onset high 
amplitude non peristaltic contractions with a completely relaxed LES. The classical findings of achalasia, incomplete LES relaxation and low amplitude aperistaltic contractions are not present in this case. However variations in the manometric features occur between patients. Rarely, complete LES relaxation and the pattern of motility seen in Diffuse Esophageal Spasm may be present as are seen in this patient. Pneumatic dilation was done and his symptoms have improved.

\section{E6}

\section{Gastroesophageal reflux symptoms during pregnancy: a longitudinal study}

R S Ramya, N Jayanthi, P C Alexander, S Vijaya, V Jayanthi. Department of Gastroenterology, Stanley Medical College, Chennai, Vinayaga Medical College, Salem and Sivarajan Maternity Hospital, Kanyakumari, Tamilnadu, India

Background: Prevalence of gastroesophageal reflux (GER) symptoms increases during pregnancy. There are very few longitudinal studies on their incidence in each trimester.

Aim: To determine the natural history of GER in pregnancy and to ascertain its risk factors.

Methods: Consecutive pregnant women attending the antenatal clinic in the I trimester were included. GER details in the three trimesters, weight record, and diet details were noted. Controls were pregnant women without reflux symptoms.

Results: Among the 60 pregnant women, GER was present in $12 \%, 5 \%$ and $3 \%$ in the I, II and III trimester respectively. $6 \%$ had symptoms in all the three trimesters. Heartburn and regurgitation was equally present in each of the three trimesters. Weight gain during pregnancy was not associated with greater risk for GER. Spicy food increased the risk of GER in the II trimester (OR 3.5) when taken three or more times a week $(p=0.017)$. Green leafy vegetables had a protective effect on heartburn (RR 15 (95\% CI 3.52 to 63.89) when taken daily and the trend remained for those taking even once, twice or thrice a week (trend chi square $p=0.0017$ ). Conclusion: The incidence of GER decreases across the three trimesters. Spicy food increases while green leafy vegetables appear to be protective against GER across the three trimesters.

\section{E7}

\section{Effectiveness of rigiflex dilatation in achalasia}

Sujay Ray, Vijay Kumar, Kshaunish Das, J Dasgupta. Division of Gastroenterology, SDLD, Institute of Post Graduate Medical Education and Research, Kolkata

Introduction: Rigiflex balloon dilatation of esophagogastric junction, aimed at reducing resistance to flow, is an effective treatment for achalasia.
Method: During January 2008 to November 2009, 25 patients with primary achalasia underwent pneumatic balloon dilation using Rigiflex balloon dilator. All patients initially underwent graded dilation $(30 \mathrm{~mm}$ first dilation followed by $35 \mathrm{~mm}$ second dilation after 4 weeks) using a Rigiflex balloon. Patients were followed up once in 3 months if dilation was successful. Data on balloon size, number of sessions of dilation, clinical response and major complications (such as perforation) and minor complications (chest pain and gastroesophageal reflux) were retrieved from medical records or obtained by telephonic interview. Symptoms score (Eckardt score) was calculated. Balloon dilation was considered successful if total symptom score was $0-1$ at the last followup visit or telephonic contact.

Result: In the 25 patients analyzed (age: mean 40 years, range 14-68; 17 male), the mean duration of symptoms was 20 (range 4-72) months. Dysphagia to solids and liquids was the presenting symptom in $25(100 \%)$ patients, regurgitation in $16(64 \%)$, retrosternal pain in $9(36 \%)$, and weight loss in $8(32 \%)$ patients. Balloon dilation was successful in 21 (84\%) patients. In 4 (16\%) patients, dilation failed to relieve symptoms. Mean number of sessions of dilation in responders and non-responders was 1 and 2, respectively.

Conclusion: Rigiflex dialatation is a safe and effective method of management of achalasia.

\section{E8}

\section{Gastroesophageal flap valve and gastroesophageal reflux disease}

$\boldsymbol{R}$ T Narendhran, Nistala Srinivas, Piyush Somani, Niraj $R$ Sawalakhe, Amit R Gharat, Q Q Contractor, P M Rathi. T N Medical College, B Y L Nair Hospital, Mumbai 400 008, India

Aim: The endoscopic grade of the gastroesophageal flap valve (GEFV) is suggested to be a good predictor of reflux status. The aim of this study was to investigate the association of the GEFV and gastroesophageal reflux.

Methods: A total of 45 patients (31 men and 14 women) with symptoms of gastroesophageal reflux disease who underwent endoscopy, esophageal manometry and $24 \mathrm{hr}$ ambulatory ph monitoring were enrolled. GEFV was graded I through IV using Hill's classification; then, GEFV was classified into two groups: a normal GEFV group (grade I) and an abnormal GEFV group (grades II, III and IV). Findings of endoscopy, esophageal manometry, and ambulatory $\mathrm{pH}$ monitoring were compared between the groups.

Results: Eleven patients had Grade A esophagitis and one had Grade B esophagitis and 33 did not have erosive esophagitis. 23 patients had Grade 1 GEFV, 13 had Grade 2 GEFV and 9 had Grade 3 GEFV. Abnormal GEFV was significantly 
associated with an increased prevalence of Gastroesophageal reflux disease as documented by $24 \mathrm{hr}$ ambulatory $\mathrm{ph}$ monitoring. Abnormal GEFV had a sensitivity of $61.29 \%$ and specificity of $71.43 \%$ in diagnosing Gastroesophageal reflux disease with a positive predictive value of $82.61 \%$.

Conclusions: Endoscopic grading of the GEFV is easy and provides useful information about the status of gastroesophageal reflux.

\section{E9}

\section{Important role of Oct-4, an embryonic stem cell marker,} in esophageal squamous cell carcinoma development

Kim Vaiphei,* Saroj Kant Sinha, Rakesh Kochhar, Kartar Singh.

Departments of Histopathology* and Gastroenteorlogy, Postgraduate Institute of Medical Education and Research, Chandigarh 160 012, India

Introduction: Concept of cancer stem cell lies in understanding the potential of its genetic expression in tumorigenesis, tumor behavior, recurrence or resistance to therapies. Oct-4, a transcription factor and a molecular marker for germ cell tumor, little is known about its expression in somatic cancers and its efficacy as a potential biomarker for esophageal squamous cell carcinoma (ESCC).

Aim: to investigate the potential expression of Oct-4 in ESCC tissue and its relationship to various clinicopathological parameters.

Methods: Esophageal biopsies from tumor and adjoining non-cancerous mucosa in patients diagnosed to have esophageal carcinoma were taken. Tissues were characterized by subjecting to frozen section. Oct- 4 expression was examined by immunohistochemistry (IHC) and RT-PCR. Esophageal biopsies from 17 patients with portal hypertension. Correlation analyses were conducted to assess the relationship between Oct-4 expression and clinicopathological parameters.

Results: There were 61 patients of ESCC, adjoining noncancerous mucosa was available in 29, 6 with dysplasia and 23 with basal cell hyperplasia. Control had normal esophageal mucosa and did not express Oct-4. Oct-4 expression levels were higher in ESCC and dysplasia compared to matching adjacent mucosa with basal cell hyperplasia. Additionally, tumor cells showed heterogenous expression for Oct-4, and correlated with tumor differentiation status, but not with other clinical or tumor parameters.

Conclusions: Oct-4 may be a potential biomarker for the initiation, progression, and differentiation of human ESCC. Our study demonstrated, for the first time, the expression of Oct-4 in ESCC and a further clue to the involvement of embryonic genes in carcinogenesis.

\section{E10}

Is empiric therapy with fluconazole appropriate for esophageal candidiasis

K G Sajith, A K Dutta, A Chacko, D S Rani, P Padmaja, $M$ Mathews.

Department Of GI Sciences, Christian Medical College and Hospital, Vellore 632 004. Tamil Nadu, India

Background and Aims: Patients diagnosed to have esophageal candidiasis (EC) are empirically treated with fluconazole. The widespread use of fluconazole may have resulted in increased resistance of Candida to this drug. We therefore aimed to assess the prevalence of fluconazole resistance to Candida. Methods: Consecutive patients undergoing endoscopy who were suspected to have EC (adherent white mucosal plaques in esophagus) were recruited from January 2010 to June 2010 after obtaining consent. The biopsy/brush samples collected from plaques were sent for $\mathrm{KOH}$ smear, histopathology and culture. The diagnosis of EC was confirmed if pseudohyphae were seen on $\mathrm{KOH}$ smear or invasive candidiasis detected on histopathology. Species identification was performed by chrome agar and formation of germ tubes. The minimum inhibitory concentration of fluconazole for Candida was done by broth dilution method and a value of $>64 \mathrm{ug} / \mathrm{ml}$ was indicative of resistance. Results: Esophageal candidiasis was detected in 39 of the 41 patients studied. (Mean age $-51.4+12.4$ years; males $64 \%$ ). Single or multiple predisposing conditions were present in 36 patients (diabetes-12, immunosuppresants8, chronic renal failure-7, chronic liver disease-5, malignancy -5 , HIV -2 , multiple factors-6).Candida albicans was identified in thirty-eight (97.4\%) patients and Candida glabrata in one (2.6\%).Fluconazole resistance was identified in 23 of $38(60.8 \%)$ cases of C.albicans and the remaining one $(2.6 \%)$ patient who had C. glabrata.

Conclusions: Two-third of patients with EC had fluconazole resistance suggesting that fluconazole is not appropriate empiric therapy for esophageal candidiasis.

\section{E11}

Impedance monitoring for diagnosis of gastroesophageal reflux disease: A preliminary report

Renu Singh, Asha Misra, Uday C Ghoshal.

Department of Gastroenterology, Sanjay Gandhi Postgraduate Institute of Medical Sciences, Raebareli Road, Lucknow 226 104, India

Background: Impedance monitoring is potentially superior to diagnose gastroesophageal reflux disease (GERD) compared to $24-\mathrm{h} \mathrm{pH}$-metry but the data from India is scanty. 
Methods: 24 patients with GERD underwent clinical evaluation (Carlsson-Dent score), esophagogastroduodenoscopy and 24-h impedance and pH-metry (ZepHr, Sandhill Scientific, USA) and were treated with omeprazole $20 \mathrm{mg} /$ day. Comparative utility of $24-\mathrm{h} \mathrm{pH}-$ metry and impedance monitoring was evaluated.

Results: Symptoms in 24 patients (median 37.5-y, 19 to 73-y; $58 \%$ male) included: heartburn $(19,79 \%)$, regurgitation (21, $81 \%)$ and chest pain (17, 71\%). 22 (92\%) had Carlson Dent score $>6$. Endoscopy negative reflux disease, Los Angeles [LA]-A, LA-B, Barrett's esophagus were present in 11 (46\%), $6(25 \%), 6(25 \%), 1(4 \%)$ and $8(33 \%)$ had hiatus hernia. 24-h pH-metry diagnosed reflux in $14(58 \%)$ patients all of whom had abnormal impedance monitoring also. Impedance monitoring diagnosed 6 additional patients with GERD all of whom had normal $\mathrm{pH}$-metry. All patients diagnosed as GERD by either method responded to treatment. Impedance monitoring was superior to $24-\mathrm{h} \mathrm{pH}$ monitoring in diagnosis of GERD ( $83 \%$ vs. $58 \%, p=0.03$, McNemar test).

Conclusion: Impedance monitoring is superior to $24-\mathrm{h} \mathrm{pH}$ monitoring in diagnosis of GERD.

\section{E12}

\section{Outcome of endoscopic dilatation in patients with esophageal stricture at a tertiary care centre}

Ajit Kumar, Akash Chaudhary, Manoj Kumar, Padmavathy,C, AC Upadhyaya.

Department of Gastroenterology, Nizam's Institute of Medical Sciences, Hyderabad, 500 082, India

Background: Endoscopic stricture dilatation using wireguided polyvinyl bougies (Savary-Gilliard dilators) is commonly used to dilate the esophageal strictures.

Aim: To evaluate the safety and efficacy of Savary-Gilliard dilatation in patients with esophageal stricture.

Methods: Consecutive patients who underwent endoscopic dilatation using Savary Gillard dilator between June 2008 to June 2010 at Nizam's Institute of Medical Sciences, Hyderabad were included. Success was defined as achieving the luminal diameter of $15 \mathrm{~mm}$.

Results: Total number of patients who underwent dilatation was 110 . Eleven patients were excluded from the study as they had insufficient data. Ninety-nine patients were included in final analysis. There were $65(65.65 \%)$ females and 34 (34.34\%) males. Different etiologies were post radiotherapy $36(36.36 \%)$, corrosive $24(24.24 \%)$, esophageal web 16 (16.16\%), peptic stricture $12(12.12 \%)$, anastamotic stricture $8(8.08 \%)$, postcricoid carcinoma $2(2.02 \%)$, Schatzki ring 1 (1.01\%). Successful dilatation was achieved in first session in $71(71.71 \%)$ and on follow up sessions (mean no. of sessions $1.9)$ in $28(28.28 \%)$ patients. Median size of first dilator used was $9 \mathrm{~mm}$ and median maximum dilatation achieved in first session was $15 \mathrm{~mm}$. Subgroup analysis based on etiology: All esophageal web successfully dilated in first session. Mean number of sessions required for successful dilatation were 2.28 in post radiotherapy stricture, 3.08 in corrosive stricture, 1.42 in peptic stricture, 2.0 in anastamotic stricture. Minor complications like mild pain (95/95), minor bleeding (74/95) and major complications like small perforation ( 2 cases) could be managed conservatively.

Conclusion: Endoscopic dilatation is a safe and effective method for palliation of dysphagia in patients with esophageal stricture.

\section{E13}

Structural and functional changes in lungs and effect of pneumatic dilation or laparoscopic cardiomyotomy on reversal of pulmonary changes in achalasia cardia

Rajinder Parshad, ${ }^{1}$ Govind Makharia, ${ }^{2} S$ K Sharma, ${ }^{3}$ Ashu Seith, ${ }^{4}$ Anoop Saraya. ${ }^{2}$

Departments of Surgery, ${ }^{1}$ Gastroenterology, ${ }^{2}$ Department of Medicine, ${ }^{3}$ and Radio diagnosis. ${ }^{4}$ All India Institute of Medical Sciences, New Delhi 110 029, India

Background: Patients of achalasia have respiratory symptoms due to chronic micro aspiration. We recently have shown structural changes in lungs in half of patients with achalasia. We studied the effect (reversal) of either pneumatic dilatation (PD) or laparoscopic Heller's cardiomyotomy (lap HCM), on above parameters.

Methods: Thirty patients with achalasia were included in this study. Esophageal and pulmonary symptoms were recorded. Pulmonary function tests were done at baseline and at the end of 6 months. High resolution computerized tomography of chest was performed prior to treatment and repeated 6 months after treatment if found abnormal at initial evaluation.

Results: The mean age of the patients was 30.97 years and mean duration of symptoms was 22.5 months. Fifteen patients $(50 \%)$ had respiratory symptoms; nocturnal cough being the commonest in 13 (43.3\%). Thirteen patients $(43.3 \%)$ had parenchymal lung changes on HRCT. Eight patients (28.5\%) had functional abnormalities in lungs in the form of restrictive airway disease. Nineteen patients opted for Lap HCM while 11 patients underwent pneumatic dilatation. Six months after treatment, the respiratory symptoms resolved in all except 2 patients $(13.3 \%)$. Four patients $(66.6 \%)$ with active lung changes on HRCT have shown resolution at 6 months. There was improvement in functional parameters on PFT evaluation with normalization of PFT in one patient.

Conclusion: Pulmonary symptoms as well as structural and functional abnormalities are common in patients with achalasia. Treatment in the form of PD or Lap HCM improves 
pulmonary symptoms and structural/ functional changes in lungs.

\section{E14}

Evaluation of gastro-oesophageal reflux disease (GERD) in patients of chronic kidney disease

$R$ Dewan, $\boldsymbol{R}$ Goel, $R$ Nehru, $N$ P Singh, S Anuradha, $S$ Garg, N Nischal.

Maulana Azad Medical College, New Delhi 110 002, India

Background and Aim: Uremia, in chronic kidney disease (CKD) can lead to Autonomic Neuropathy (AN). AN is known to cause delayed gastric emptying, impaired esophageal motility and increase in transient lower esophageal sphincter relaxation. Increased gastric acid production in uremia is known. The present study investigated the patients of CKD for autonomic dysfunction, GERD, esophageal dysmotility and relationship between them.

Methods: 53 patients of CKD were selected after excluding those on drugs or having diseases known to affect GI motility and LES tone. All were evaluated for reflux symptoms and underwent UGI endoscopy, $24 \mathrm{hr}$ pH-metry, esophageal manometry and a battery of bed side autonomic function tests. Severity of CKD was staged according to K/DOQI classification. Results: Symptomatic GERD was seen in $44(83.01 \%)$ patients. Significant reflux on $\mathrm{pH}-$ metry was seen in 41 patients $(77.35 \%)$. Patients with advanced CKD stage had significant reflux $(p<0.05)$. Endoscopic esophagitis was documented in 34 cases $(64.15 \%)$. Esophageal motility was abnormal in 25 cases $(47.16 \%)$. Autonomic dysfunction was present in 28 patients $(52.83 \%)$ ). . A higher percentage of cases with esophageal dysmotility had autonomic neuropathy as well $(p<0.05)$. A significant correlation was observed between autonomic neuropathy and GERD $(p=0.000)$. Patients with GERD had significant esophageal dysmotility $(p=0.000)$. Conclusion: GERD is common in CKD patients and it correlates with the severity of the kidney disease. In the presence of autonomic dysfunction, treatment for GERD in these patients may also require treatment for esophageal dysmotility.

\section{Stomach-non H. pylori}

\section{ST1}

\section{Evaluation of upper gastrointestinal endoscopy in dyspepsia in relation to age and alarm symptoms}

Thayumanavan, Nithya, Jegannathan, Kannan, Ramani, Hari. Department of Gastroenterology, Madurai Medical College, Madurai 600020
Aim: To study the value of upper gastrointestinal endoscopy in patients with new onset previously uninvestigated dyspepsia in relation to age and presence of alarm symptoms.

Methods: Upper gastrointestinal endoscopy was performed with a flexible fibreoptic endoscope in 224 patients with new onset dyspepsia after enquiring into alarm symptoms and appropriately ruling out structural disease with ultrasonogram of the abdomen. The mucosa of the stomach and pylorus was analyzed for any lesions. Correlation between age, alarm symptoms and mucosal lesions was done.

Results: In patients 50 years, 14 out of 23 patients $(60.9 \%)$ who had alarm symptoms had serious lesions on endoscopy including gastric malignancy. Fifty five out of 63 patients $(87.3 \%)$ without alarm symptoms had no endoscopic findings.

\section{ST2}

\section{Squamous cell carcinoma of stomach: Case report}

D Sasi Anand, A Chezhian, S Arul Prakash, R Bala Murali, G Ram Kumar, S Chitra, K Muthu Kumaran, T Pugazhendhi, $S$ Jeevan Kumar.

Department of Digestive Health and Diseases, Government Kilpauk Medical College, Chennai 600 010, India

A 60-year-old female presented with complains of pain abdomen, non-bilious vomiting for two weeks. She was treated for well differentiated squamous cell carcinoma esophagus lower third 7 months ago. She underwent tanshiatal esophagectomy, gastric pull through and esophago-gastric anastomosis. Patient remained asymptomatic for 7 months. Ultrasound abdomen and CT scan thorax and abdomen did not reveal any metastasis at initial presentation. Endoscopic examination showed an excavating ulcer in the gastric conduit. Histopathology showed malignant squamous epithelial cells, keratin pearl formation, surrounded by gastric cells suggestive of well differentiated squamous cell carcinoma. She was given chemotherapy (5-FU), and patient died after 2 months of treatment.

Discussion: The most frequent site of visceral metastases of SCC esophagus is the liver, followed by the lung and adrenals. Intramural metastasis is defined as a metastatic tumor in the oesophageal or gastric wall arising from a primary lesion in the oesophagus. Metastasis is induced mainly via extension by way of the submucous lymphatics Treatment modality includes surgical resection, radiation therapy, chemotherapy, or combination of these modalities. Tumor limited to stomach and $<2 \mathrm{~cm}$ in diameter, surgical resection should be performed, for tumor $>2 \mathrm{~cm}$, surgery is palliative in most patients. We report this case of squamous cell carcinoma of esophagus with intramural metastasis to stomach. 


\section{ST3}

Primary gastric tuberculosis causing gastrocolic fistula in an immunocompetent individual: A case report

B Ravi Shankar, T L V D Prasad Babu, G R Srinivas Rao, Ambareesh Barat, $K B$ Kailash, Vamsi Krishna, Jithendar Reddy.

Departments of Gastroenterology and Gastrointestinal Surgery, Yashoda Hospital, Secunderabad

Background: Gastrocolic fistula is an uncommon complication of benign and malignant gastric disease. We present an unusual case of gastrocolic fistula due to primary gastric tuberculosis.

Case Presentation: A 50 year old male agricultural worker presented with complaints of low grade fever for 6 months, and watery diarrhoea, faeculent vomiting and weight loss of about 6-7 kg since 1 month. He was malnourished with a BMI of 17.1. Hemoglobin was $11.5 \mathrm{gm} / \mathrm{dl}$ and serum albumin was $3.5 \mathrm{gm} / \mathrm{dl}$. Upper gastrointestinal endoscopy and colonoscopy were suggestive of gastrocolic fistula, however, the biopsies taken were not conclusive. The patient underwent a barium enema and CECT abdomen which delineated the fistula. CECT also showed a lymphnodal mass near the celiac axis. He was operated upon, and histopathology of tissues obtained during operation was suggestive of tuberculosis.

Conclusion: Tuberculosis needs to be considered in the Indian setting, as a possible cause of gastrocolic fistula.

\section{ST4}

\section{Response to endoscopic dilatation in corrosive versus peptic gastric outlet obstruction}

$M T$ Noor, $R$ Kochhar, $P$ Dixit, $K S$ Poorna, U Dutta, $B$ Nagi, K Singh.

Department of Gastroenterology, Postgraduate Institute of Medical Education and Research, Chandigarh 160 012, India

Background: Corrosive injury and peptic ulcer disease are the leading causes of benign gastric outlet obstruction (BGOO). We compared the clinical profile and endoscopic management of these patients.

Methods: Clinical and endoscopic data of patients with BGOO treated by us from 2002-2010 were reviewed. Endoscopic management was done using TTS, CRE balloons with the target diameter of $15 \mathrm{~mm}$ every 2 weeks. Local steroid therapy or needle knife incision was used to augment the effect of dilatation. Comparison of results between corrosive and peptic BGOO was done. All patients were followed up for a minimum of 6 months.
Results: A total of 57 patients of corrosive and 47 patients of peptic etiology were studied. Peptic group had higher age $(51.8 \pm 16.1$ vs. $34.5 \pm 12.3$ years, $p<0.001)$, longer duration of symptoms [median $6(1-120)$ vs 2 (0-24) months, $p<0.001]$. Forty of 47 patients with peptic BGOO were H. pylori positive and underwent successful eradication. Number of sessions to achieve target diameter was more in the corrosive group as compared to peptic group $(6.1 \pm 2.9$ vs $2.7 \pm 1.8, p<0.001)$. Requirement of local steroid was more common in the corrosive group as compared to peptic group [7(12.3\%) vs $0(0 \%), p=0.013]$. Surgery was required only in 4 patients in the corrosive group. Symptom relief was similar in the two groups. There was only one major complication in the form of perforation in corrosive group.

Conclusion: Patients with BGOO due to corrosive injury require more dilatation sessions and longer duration of treatment than peptic GOO.

\section{ST5}

\section{A study on post gastrojejunostomy cases}

P Subramanian, S Jeevan Kumar, T Pugazhendhi, $R$ Balamurali, S Chitra, G Ramkumar, K Muthukumaran, $S$ Babukumar.

Department of Digestive Health and Diseases, Government Peripheral Hospital, Anna Nagar, Chennai 600102, India

Aim: To observe the clinical and endoscopy findings in Post gastrojejunostomy cases.

Methods: Data were collected from UGI endoscopic registers and DDHD notebooks of Post gastrojejunostomy patients who attended our department between August 2008 and July 2010.

Results: (i) Number of patients studied: 94 (ii) cause for surgery: corrosive injury stomach:9 (9.57\%) peptic ulcer complication: 67 (71.27\%) malignancy: 18 (19.15\%) (iii) predominant symptoms: pain abdomen: 42 (44.68\%) vomiting:18 (19.15\%) dysphagia: $13(13.83 \%)$ regurgitation:13 (13.83\%) bloating:16 (17.02\%) anorexia:21 (22.34\%) weight loss:23 (24.47\%) early satiety:8 (8.51\%) malena: 9 (9.57\%) diarrhea:13 (13.83\%) ball rolling movements:6 (6.38\%) jaundice:5 (5.32\%) (iv) clinical signs: anemia:56 (59.57\%) angular stomatitis:7 (7.44\%) oral thrush:4 (4.25\%) koilonychia:5 (5.32\%) icterus:5 (5.32\%) cachexia:9 (9.57\%) (v) endoscopy findings: both loops entered:71 (75.53\%) only one loop entered: $23(24.47 \%)$ peristomal inflammation / erosion / ulcer / edema: 18 (19.15\%) gastrojejunocolic fistula: $1(1.06 \%)$ peristomal carcinoma in previously benign cases:3 (3.19\%) postcricoid web:7 (7.44\%) esophagitis:10 (10.64\%) bile in stomach / bile gastritis:36 (38.30\%) gastric bezoar:5 (5.32\%) gastric polyp:4 (4.25\%). 
Conclusion: (1) pain abdomen followed by weight loss, anorexia and vomiting are the common symptoms while anemia is the most common sign observed in post gastrojejunostomy cases. (2)bile in stomach is the commonest endoscopic finding followed by peristomal lesions. (3) carcinoma developed in $3.19 \%$ of previously benign gastrojejunostomy cases.

\section{ST6}

\section{Gastric polyps-An audit}

B Prakash Shankar, P Jagadesan, G Ramkumar, K Muthukumaran, T Pugazhendhi, S Jeevan Kumar.

Department of Digestive Health and Diseases, KMC, Chennai, India

Aim: To study the incidence, age distribution, symptomatology, site of distribution and histology of gastric polyps. Methods: Study period 6 months (December 2009 to June 2010) Study population 1056 patients who underwent oesophagogastroduodenoscopy for various reasons. Age distribution 25 years to 80 years. Study involved assessment of various characteristics of gastric polyps. Polyps less than $0.5 \mathrm{~cm}$ were termed as diminutive polyps and biopsy was not done. Patients were advised regular follow up. Polyps with size $>1 \mathrm{~cm}$ were subjected to biopsy and histopathological examination.

Results: Overall incidence 2.84\% Total number of patients with gastric Polyps 3015 polyps were diminutive and 15 polyps were large $(>1 \mathrm{~cm}) 6$ polypectomies done. Age: average $50 \pm 0.63$ years. sex ratio: male: female - 1: 1.2 larger polyps common in males $2: 1$ presenting symptoms: gastric outlet obstruction: 2 dyspepsia:10 heart burn:2 ugi bleed:3 site of polyps: fundus: 5 antrum:5 body: 2 lesser curvature: 2 pylorus:3 biopsy report: hyperplastic polyp: 12 adenomatous polyp: 4 carcinoid: 1 .

Conclusion: Gastric polyps are not uncommon (incidence rate 3\%). Hyperplastic polyps are common in our scenario. Predominant site of polyps is fundus and antrum. Hyperplastic polyps are common in antrum. Presenting symptoms vary from simple dyspepsia to gastric outlet obstruction. Polypectomy is a safe procedure with no adverse effects.

\section{ST7}

\section{Use of $99 \mathrm{mTc}$-pertechnate scintigraphy in assessment of gastric mucosal viability after acute corrosive injury}

Amit Bhoil, C N B Harisankar, Anish Bhattacharya, $B$ R Mittal, U Dutla, K Singh, R Kochhar.

Departments of Nuclear Medicine and Gastroentrology, Postgraduate Institute of Medical Education and Research, Chandigarh 160 012, India
Aims: To non-invasively assess the gastric mucosal viability after corrosive injury using 99m-Technetium scintigraphy. (99mTc-S).

Methods: Consecutive patients with corrosive ingestion undergoing endoscopic evaluation were subjected to $99 \mathrm{mTc}-\mathrm{S}$ after injection of $5 \mathrm{mCi}$ of pertechnetate (Tc$99 \mathrm{~m})$. Hybrid SPECT/CT of the abdomen was performed with stomach divided into proximal and distal segments. $99 \mathrm{mTc}-\mathrm{S}$ uptake patterns were divided into good uptake and impaired/ no uptake. Findings of $99 \mathrm{mTc}-\mathrm{S}$ were compared with endoscopic grading into those with injury $<$ Gr II b and Gr IIb.

Results: 14 patients (10 male), age 17-60 yrs were included into the study. 1 died before scintigraphy could be preformed. On endoscopy, 9 regions had gastric mucosal injury of grade $<$ IIb ( 7 in proximal stomach, 2 in distal stomach). $99 \mathrm{mTc}$ uptake was good at all the corresponding sites. 15 regions had injury of grade IIb (5 proximal stomach, 10 distal stomach). Of them, 14 had no $99 \mathrm{mTc}$ uptake at the corresponding sites. 1 patient having discordant finding had $99 \mathrm{mTc}$ uptake done after 1 month of the day of ingestion of corrosive substance. 1 patient had no $99 \mathrm{mTc}$ uptake along the lesser curvature and endoscopy showed Gr IIIa injury of the corresponding region.

Conclusion: Significant positive correlation was noted between endoscopic findings and $99 \mathrm{mTc}-\mathrm{S}$ findings. $99 \mathrm{mTc}-\mathrm{S}$ can be used to differentiate between mucosal injury of grade $<$ IIb and IIb non-invasively. $99 \mathrm{mTc}-\mathrm{S}$ can be valuable in patients not fit for endoscopy.

\section{ST8}

Symptom response with long term and short term antisecretory drugs (H2RA and PPI) in dyspepsia

\section{R S Ramya, P C Alexander, K Janani, V Jayanthi.}

Department of Gastroenterology, Stanley Medical College Hospital, Chennai 600 003, India

Background: Dyspepsia symptoms often requires long term anti secretory drugs for relief of symptoms.

Aim: To determine relief of dyspepsia symptoms in patients on long and short term H2RA and PPI at the end of 1 and 6 months.

Methods: Patients with ulcer, reflux or dysmotility type dyspepsia (normal endoscopy, $\mathrm{H}$ pylori negative) and on H2RA or PPI for more than a month were included. The baseline symptom profile of the patients was noted. The symptoms were reassessed at end of one month and 6 months, while on treatment.

Results: 59 patients (Gp I) were on long term anti secretory drugs (mean: 3 years). There was no significant differences 
in symptom responses in ulcer type, dysmotility and reflux type dyspepsia at end of one and 6 months with either H2RA (in 31 patients) or PPI (in 28 patients). $88 \%$ had recurrence of symptoms within a week on discontinuation of the drugs. In Gp II, the mean duration amongst the 49 nave patients each on H2RA (Gp IIa) or PPI (Gp II b) was 5 months. At the end of one month, $40 \%$ of ulcer type, $31 \%$ for reflux type and $24 \%$ for dysmotility type had relief of symptoms which did not increase significantly at 6 month ( $45 \%$ vs. $29 \%$ vs. $23 \%$ ). The trend was similar in those on PPI at one and 6 months.

Conclusion: The initial response with H2RA / PPI is significant with short term treatment, but is not sustained in those on long term treatment.

\section{Stomach-H. pylori}

\section{STH1}

\section{Prevalence of Helicobacter pylori in relation to socioeconomic status}

Thayumanavan, Nithya, Jegannathan, Kannan, Ramani, Hari.

Department of Gastroenterology, Madurai Medical College, Madurai 600 020, India

Background: H. Pylori infects people worldwide and is generally associated with unhygeinic conditions and poor socioeconomic status. We studied the prevalence of H. Pylori in dyspeptic subjects according to their socioeconomic status. Aim: To study the prevalence of Helicobacter pylori in relation to socioeconomic status.

Methods: A total of 224 patients with new onset dyspepsia were included in the study. They were classified according to the socioeconomic status using the Modified Kuppuswamy classification which utilizes three parameters namely education, occupation and monthly income. Socioeconomic status score was assigned to each patient. Upper gastrointestinal endoscopy was performed in all patients and antral mucosal sample was obtained for ultra rapid urease reaction for detection of Helicobacter pylori.

Results: Out of 224 patients, 21 (9.3\%) were from the lower socioeconomic class, 85 (37.9\%) from the upper lower class, 91 (40.6\%) from lower middle class and 27 (12\%) from the upper middle class. The lower and upper lower classes were combined together as lower socioeconomic status (47.4\%) and the lower middle and upper middle classes were combined together as middle socioeconomic status $(52.6 \%)$. In the middle socioeconomic status, 54 out of 118 (45.8\%) tested positive when compared with the lower socioeconomic status in which 78 out of 108 (73.6\%) were positive.

\section{STH2}

\section{Prevalence of $\boldsymbol{H}$. pylori in dyspepsia and smokers}

L Thayumanavan, Nithya Sridharan, M Kannan, R Ramani, A S A Jagannathan.

Department of Gastroenterology, Madurai Medical College, Madurai 600 020, India

Background: Functional dyspepsia is the commonest cause for medical consultation whorld wide. H. Pylori also infects more than $50 \%$ of the population. We studied the prevalence of H. Pylori in our dyspeptic patients and compared them with published literature. Also smoking has a profound in fluence on symptomatology. We also studied the difference in H.pylori prevalence in smokers and non smokers.

Aim: To see the prevalence of H.pylori in dyspeptic patients. To compare our findings with published literature. To study the impact of smoking on H. pylori.

Methods: Patients attending our hospital with dyspeptic symptoms from Aug 2008 to May 2009 were included. A detailed history, examination, CBC, blood biochemistry, ultrasound, endoscopy were done in all patients. History of PPI, antibiotic use within 2 weeks was specifically taken as exclusion criteria.Details of smoking habits were taken. $H$. pylori was detected using the RUT.

Results: 173/224 were classified as functional dyspepsia (77.2\%).There were 135 males and 89 ladies in the age group 18 to 72 (mean $44.6+14.6$ yrs). Peptic ulcers were seen in $7.5 \%$, CA stomach in $4 \%$, erosive gastritis in $5.8 \%$, miscellaneous 3.5\%. 102/173 (59\%) were positive for H. pylori. Amongst the various age groups showing H.Pylori positivity, $25 / 36$ were $>60$ years $(70 \%)$, and61.7\% in 30 to 39 years. There were 29 smokers. H.pylori was detected in 24 (82.8\%). Conclusions: H.pylori was seen in $59 \%$ of our subjects with NUD. This compared well with published literature. $H$. pylori was significantly higher in the smokers.

\section{STH3}

\section{Changing efficacy of triple therapy for eradication} of $\boldsymbol{H}$. pylori

$G N$ Mishra, A K Jain, G Nath, V K Dixit, S K Shukla, P Behera.

Department of Gastroenterology, and Microbiology, Institute of Medical Sciences, Banaras Hindu University, Varanasi 221 005, UP, India

Background: Antibiotics and PPI based triple therapy is of choice for eradication of $H$. pylori, a bacterium causing chronic gastritis, peptic ulcer and predisposing to cancers. However antibiotic resistance is becoming frequent. 
Aim: To find H. pylori eradication rate using clarithromycin (500 mg bid), amoxicillin (750 mg bid) and pantoprazole (40 $\mathrm{mg}$ bid) and compare it with eradication rate 10 years earlier.

Method: 22 patients (antral gastritis 11, DU 9 and GU 2) who were $H$. pylori positive completed 2 weeks drug therapy and reported for assessment of eradication at 6 weeks.

Results: Eradication was documented by culture and RUT negativity in 5/22 (23\%) patients only. All these patients improved symptomatically and endoscopically. Thirteen of the remaining 17 improved endoscopically and clinically RUT was negative in $15 / 17$. Culture was positive in all 17 and was confirmed to be $H$. pylori by PCR. This eradication rate was much lower than that obtained a decade earlier $(89 \%)$ in a similar population. However, ulcer healing was comparable ( $78 \%$ vs. $96 \%$; $p=\mathrm{NS}$ ) Conclusion: Triple therapy was unable to eradicate $H$ pylori infection in three fourth of patients.

\section{STH4}

\section{Prevalence of cagA and vacA genotypes of $\boldsymbol{H}$. pylori as a putative virulence marker in dyspeptic patients}

P Dixit, A K Sharma, K K Prasad, S K Sinha, C K Nain, $K$ Singh.

Department of Superspeciality of Gastroenterology, Postgraduate Institute of Medical Education and Research, Chandigarh 160 012, India

Background: The cytotoxin associated gene A (cagA) and the vacuolating cytotoxin gene A (vacA) of $H$. pylori has been associated with phenotypic characteristics of virulence contributing to mucosal damage.

Objective: To know the prevalence of virulent genes cagA \& vacA in patients of functional dyspepsia, duodenal ulcer and GERD attending Nehru Hospital, PGIMER, Chandigarh.

Methods: A total of 40 patients of functional dyspepsia (FD), duodenal ulcer (DU) and GERD were included in the study. All patients underwent UGEI. Four antral biopsies were collected for RUT, genomic DNA isolation and histology.Isolated genomic DNA from biopsies were subjected to PCR for cagA,vacAs1 and vacAs2 genes.

Results: The mean age of pts. (M 21, F 19) was $42.9+$ 13.7 (range 22-65 yrs.). RUT was positive in $8 / 26$ patients $(30.7 \%)$ of DU. Among them cagA gene was detected in 10 pts. (38.4\%).Among 26 patients of DU vacAs1 was detected in 7 patients $(27 \%)$ and vacAs2 in 3 pts. (11.5\%). Out of 6 patients of functional dyspepsia 1 patient was RUT positive. In 2 patients of FD cagA gene was detected while vacAs1 and vacAs2 were detected in 2 patients 2 samples of GERD were +ve for RUT. CagA gene was detected in 2 patients of GERD. In one patient vacAs1 was detected while vacAs2 was detected in another patient.

Conclusion: The prevalence of cagA and vacAs1 genes of H.pylori was more in patients of duodenal ulcer. Patients infected of H.pylori genotype cagA, vacAs1 may be associated with increased risk of acquiring peptic ulcer disease in our population.

\section{STH5}

\section{Prevalence of Helicobacter pylori in patients with peptic} ulcer disease: A clinic based study

Sanjeev K Thakur, Sanjeev Jha, Utpal Anand, Vijay Prakash. Bihar Institute of Gastroenterology, Patna, Bihar

Aim: To evaluate the prevalence of $H$. pylori in our patients with peptic ulcer disease (PUD).

Methods: A prospective study of consecutive patients with PUD. The diagnosis was based on history and endoscopy. Biopsy from antrum was done using endoscopic biopsy forceps (cusp diameter $5 \mathrm{~mm}$; Boston Scientific). Specimen was immediately placed on RUT kit (Allied marketing Corp. Calcutta). The results were read after $30 \mathrm{~min}$ as per manufacturer's instruction. Those who tested positive for $H$. pylori were classified as group A and those negative as group B. The two groups were compared for their baseline characteristics. Patients with coagulopathy, active bleeding, pregnancy or those on aspirin or anticoagulants were excluded.

Results: During the study duration (June 09 to June 10) 108 patients (79 men; age $45.1+16.4 \mathrm{yrs}$ ) of PUD fulfilled the inclusion crieteria. group A comprised of $28 / 108$ patients (25.9\%) who tested positive for H.pylori (20 men; age 45.07+16.15: range 20-70 yrs). Group B comprised of $80 /$ $108(74.07 \%)$ patients who tested negative (59 men: age $45.7+15.42$ yrs. In both the groups DUs were twice as common as gastric ulcer in both the groups $(10 / 28$ (37.03\%: group A) vs. 27 / 80 (33.7\%: group B). There was no significant difference in the two groups according to age, sex or, type of lesion $(p=n s)$.

Conclusions: H.pylori prevalence in patients with peptic ulcer disease is much lower than reported in our clinic based experience.

\section{STH6}

Helicobacter pylori associated refractory iron-deficiency anemia

M R Ajmal, P P Abdul Shahid, Shamshad Ahmad. Departments of Medicine and Pathology, J N Medical College, Aligarh Muslim University, Aligarh 202 002, UP, India 
Background: H. pylori infection has been reported as a cause in refractory iron deficiency anemia (RIDA) and eradition of $H$. pylori improves RIDA.

Aim: We undertake this study to know the quantum of $H$. pylor $i$ associated IDA and effect of $H$. pylori eradication in Aligarh region for the first time.

Method: We selected 60 cases of RIDA, having no obvious cause of anemia, $H$. pylori positive by RUT and histological examination. They were divided in three groups Group 1 $(n=20)$ received only anti H.pylori therapy for two weeks Group $2(n=20)$ received oral iron therapy for three months and anti-H.pylori in second month Group $3(n=20)$ received oral iron therapy for three months and anti- H.pylori in second month.

Results: In Gr. 1, Gr. 2 and Gr.3, mean baseline hemoglobin was $6.78 \pm 1.32 \mathrm{gm} \%, 6.99 \pm 1.84 \mathrm{gm} \%$ and $6.61 \pm 1.32 \mathrm{gm} \%$ respectively, mean baseline S. iron level was $35.80 \pm 10.38$ $\mathrm{mg} / \mathrm{dl}, 36.10 \pm 10.60 \mathrm{mg} / \mathrm{dl}$ and $38.65 \pm 7.44 \mathrm{mg} / \mathrm{dl}$ respectively, mean base line S. ferrotin was $11.45 \pm 1.70 \mathrm{mg} / \mathrm{ml}, 12.70 \pm$ $2.17 \mathrm{mg} / \mathrm{ml}$ and $12.05 \pm 1.60 \mathrm{mg} / \mathrm{ml}$ respectively. At the end of 3 months in Gr 1, Gr.2 and Gr.3 mean rise of hemoglobin was $4.36 \mathrm{gm} \% ; 5.56 \mathrm{gm} \%$ and $1.85 \mathrm{gm} \%$ respectively, mean rise in s. iron level was $18.35 \mathrm{mg} / \mathrm{dl}, 37.75 \mathrm{mg} / \mathrm{dl}$ and $9.9 \mathrm{mg} / \mathrm{dl}$ respectively.

Conclusion: H. pylori is significant cause of RIDA and anti H.pylori treatment significantly improved RIDA.

\section{STH7}

\section{Is PCR-RFLP technique reliable for $\mathrm{mEH}$ genotype in gastric cancer?}

Virendra Jaiswal, ${ }^{1}$ Ujjala Ghoshal, ${ }^{1 *}$ Sushil Kumar, ${ }^{2}$ Shweta Tripathi, ${ }^{2}$ Balraj Mittal, ${ }^{3}$ Uday C Ghoshal. ${ }^{2}$

${ }^{1}$ Departments of Microbiology, ${ }^{2}$ Gastroenterology and ${ }^{3}$ Genetics, Sanjay Gandhi Postgraduate Institute of Medical Sciences, Lucknow 226 014, India

Background: We aimed to study the role of microsomal epoxide hydrolase (mEH) exon 3 His113Tyr, an important gene encoding for Phase-II xenobiotic enzyme, in patients with gastric cancer (GC) as compared to peptic ulcer (PU) and healthy controls (HC) by PCR-RFLP and compare the results of PCR-RFLP with sequencing.

Methods: 85 patients with GC, $55 \mathrm{PU}$ and $160 \mathrm{HC}$ were genotyped by PCR- RFLP and compare the results of PCRRFLP of $64 \mathrm{GC}, 47 \mathrm{PU}$ and $88 \mathrm{HC}$ by direct sequencing. $H$. pylori diagnosis was confirmed using any two of three tests, rapid urease test (RUT), histopathology and anti-H. Pylori $\mathrm{IgG}$ antibody by ELISA.

Results: Patients with GC 54.4 \pm 11.8 years, 62 [72.9\%] men, PU 51.5 $\pm 14.4,41$ [74.5\%] and HC $(52.9 \pm 11.4,118$ [73.8\%]) were comparable with respect to age and gender.
Reliability of PCR-RFLP technique by direct sequencing had shown wild type $\mathrm{T} / \mathrm{T}(\mathrm{Kappa}=0.990)$ and heterozygous $(\mathrm{C} / \mathrm{T})$ had high concordance $(0.807)$ while homozygous $(C / C)$ had substantial discordance $(0.640)$ with $p$ value $<0.05$. None of the genotype were associated with GC as compared to $\mathrm{HC}$ either by PCR-RFLP or sequencing His113His $(P=0.221$, $\mathrm{OR}=0.583,95 \% \mathrm{CI}=0.245-1.385)$ or His $113 \mathrm{Tyr}(P=0.090$, $\mathrm{OR}=0.489,95 \% \mathrm{CI}=0.207-1.122)$ and $\mathrm{His} 113 \mathrm{His}$ $(P=0.643, \mathrm{OR}=1.301,95 \% \mathrm{CI}=0.408-3.958)$ or His $113 \mathrm{Tyr}$ $(P=0.784, \mathrm{OR}=1.171,95 \% \mathrm{CI}=0.378-3.624)$ respectively. Similar results were found in $\mathrm{GC}$ versus PU and PU versus HC. H. pylori had shown significant association with PU rather than GC $(p<0.001)$.

Conclusions: Significant discrepancy was observed in mEH3 His113Tyr genotype; hence direct sequencing should be preferred over PCR-RFLP.

\section{STH8}

Incidence of $\boldsymbol{H}$. pylori infection with associated intestinal metaplasia and gastric atrophy in dyspeptic patients with endoscopic evidence of antral gastritis. A prospective study

A K Agasti, S Smita, ${ }^{@}$ Milind Patil, ${ }^{@}$ P Jignesh, M Nasa, Prabha Sawant.

Departments of Gastroenterology and @ Pathology, Lokmanya Tilak Muncipal Medical College and General Hospital, Sion, Mumbai 400 022, India

Aim: To study the Incidence of H. Pylori infection, intestinal metaplasia and gastric atrophy in dyspeptic patients with endoscopic evidence of antral gastritis.

Methods: A total number of 200 patients, with dyspepsia were followed up after 4 weeks of PPI therapy. Patient who were still symptomatic underwent OGD scopy. Patients with antral gastritis on OGD scopy, biopsy was taken from antrum and body for RUT (Rapid urease test) and histopathology. Those with history of NSAID use, duodenal ulcer, reduced gastric folds, pangastritis and esophagitis on OGD were excluded.

Result: One hundred and forty-six patients were non responders to PPI, 90 patients had endoscopic evidence of antral gastritis. 50 (55.55\%) patients were positive for H. Pylori on histopathology and $45(50 \%)$ were RUT positive. Intestinal metaplasia found in 15 and gastric atrophy in 35 patients reflecting a incidence of 30 and $70 \%$ respectively. All patients were treated with 3 drug regimen for $H$. pylori for14 days.

Conclusion: Incidence of H.pylori is around $55 \%$ in dyspeptic patients who had only antral gastritis on OGD scopy with $30 \%$ of them having intestinal metaplasia and $70 \%$ having gastric atrophy in our study. The diagnostic accuracy of RUT is comparable with histopathology for detecting H. pylori. 


\section{STH9}

\section{COX 2 expression and its association with $H$. pylori} in carcinoma stomach

Puneet, Shashi Prakash, V K Dixit, Mohan Kumar, Gopeshwar Narayan, Gopal Nath, A K Khanna.

Departments of General Surgery, Gastroenterology, Pathology, Molecular and Human Genetics, and Microbiology, Banaras Hindu University, Varanasi

Introduction: Gastric cancer constitutes $9 \%$ of all cancers and second most common cause of cancer deaths worldwide. Besides other factors $H$. pylori documented to be important carcinogen which increases risk of gastric cancer. Upregulation of COX- 2 expression has been correlated with development of gastric cancer.

Objective: Study planned to look for COX-2 expression in gastric carcinoma, its association with clinicopathological characteristics and H. pylori.

Methods: 40 gastric cancer patients (24 males, 16 females; mean age $51.92 \pm 13.06$ years) and 13 controls (10 males, 3 females; mean age $53.53 \pm 12.56$ years) taken in study. Biopsies taken following either gastric resection or UGI endoscopy and subjected for histopathological examination, COX-2 expression, H. pylori culture and rapid urease test. COX-2 expression studied with PCR using DNA polymerase and specific primers. H. pylori detection done by rapid urease test and culture. Histopathological examination done for confirmation of diagnosis.

Results: In our study, 65\% samples had upregulation and $35 \%$ showed no significant change in COX-2 expression pattern. No significant correlation found between COX-2 expression with tumour stage, lymph node involvement and tumour histopathology. H.pylori found positive in 15 (37.5\%) patients of carcinoma stomach and 9 (69.2\%) normal patients. Mean of expression of COX-2 gene was higher in H.pylori positive cases.

Conclusion: COX-2 expression was upregulated in gastric cancer cases and expression level is significantly higher in H. pylori positive cases showing possible aetiological role of H.pylori over COX-2 expression in gastric cancer.

\section{STH10}

Association between Helicobacter pylori and alcohol use in urban population study from a tertiary care center

\footnotetext{
K Vishnu Vardhan, ${ }^{* *}$ C Akash, ${ }^{* *}$ S Rajanikanth,${ }^{*}$ V Shanthi, ${ }^{*}$ $S$ Shanmuganathan.

** Department of Gastroenterology, Nizam's Institute of Medical Sciences, Hyderabad 500 082, * Department of Medical Gastroenterology, Sri Ramachandra Medical College, Chennai, India
}

Introduction: Helicobacter pylori is a well recognized cause of peptic ulcer disease, chronic gastritis and gastric cancer. Many factors affect the acquisition and elimination of $H$. pylori in adults. One of the factors which is of interest is alcohol intake. Alcoholic beverages are known to have multiple direct and indirect effects on the gastric mucosa, gastric emptying, gastric acid secretion and have strong direct antibacterial activity. Most of the studies done so far showed an inverse relation of alcohol intake with $H$. pylori.

Aim: To study the relationship between alcohol consumption and Helicobacter pylori infection.

Methods: Consecutive patients referred to endoscopy for dyspeptic symptoms were enrolled in to the study. Patients consuming $>70 \mathrm{gm}$ of alcohol per week $(n=50)$ were compared with nonalcoholics $(n=50)$. Patients with history of treatment for H. pylori, recent antibiotic or PPI intake, gastric malignancy and ulcer surgery were excluded. Endoscopic biopsy was taken from antrum and corpus and rapid urease test was done.

Results: RUT was positive in $46 \%$ (23) patients in alcohol group and 74\% (37) patients in control group with a $p$ value of.04. The mean duration of alcohol consumption is $11.74 \mathrm{yrs}$ in patients who are positive for $H$. pylori as compared to 9 yrs in patents who are negative with no statistically significant deference.

Conclusion: The prevalence of $H$. pylori is low among people consuming alcohol regardless of the type of alcoholic beverage and the duration of consumption.

\section{STH11}

\section{Evaluation of utility of pepsinogen (PG)-I, PG-II and gastrin-17 serum levels in diagnosis of cancer-associated gastric mucosal lesions like intestinal metaplasia in patients with $\mathrm{H}$. pylori infection in India}

\section{Uday C Ghoshal, Sushil Kumar, Narendra Krishnani," Dipti Chourasia, Shweta Tripathi. \\ Departments of Gastroenterology and Pathology, ${ }^{*}$ Sanjay Gandhi Postgraduate Institute of Medical Sciences, Raebar- eli Road, Lucknow 226 014, India}

Background: Intestinal metaplasia (IM), a precursor of gastric cancer (GC), though can be detected on histology, ratio of serum pepsinogen (PG)-I/PG-II is potentially useful; hence, role of it and of gastrin-17 (G-17) to detect IM in patients with $H$. pylori infection was evaluated.

Methods: 98 patients with GC and 62 with dyspepsia were evaluated by endoscopy, histology for IM (H\&E, PAS, Alcian blue stains) and H. pylori (H\&E, Giemsa stain), rapid urease test and $\mathrm{IgG}$ antibody. Positive result in any 
two tests was diagnostic of $H$. pylori infection. Serum levels of PG-I, PG-II and G-17 were estimated using commercial ELISA (Biohit, Finland).

Results: Patients with GC were comparable to dyspepsia in age and gender. $29(30 \%)$ patients with GC and $33(54 \%)$ with dyspepsia had H. pylori $(p=0.004)$ and $35(36 \%)$ with GC and $9(14 \%)$ with dyspepsia had IM $(p=0.004)$. Patients with IM $(n=44)$ had lower PG-I/PG-II ratio than those without $(n=116$; median $4.4,0.37-23.6$ vs. $6.3,0.19-38.6, p=$ $0.005)$. The cut-off PG-I/PG-II ratio of 6.0 had sensitivity $64 \%$ and specificity of $52 \%$ to detect IM on ROC curve (AUC 0.64). 26/44 (60\%) patients with IM and 52/98 (53\%) with GC had P ratio less than 6. G-17 was comparable among patients with and without IM ( $12.1 \mathrm{pg} / \mathrm{L}, 0.5-40.6$ vs. $11.3 \mathrm{pg} / \mathrm{L}, 0.65-37.1, p=\mathrm{ns})$.

Conclusion: Though PG-I/PG-II ratio is lower in patients with IM, only $60 \%$ patients with IM had low PG-I/PG-II ratio suggesting that this test may not be useful in noninvasive diagnosis of IM in Indian population.

\section{STH12}

\section{Determination of Epstein-Barr virus load and its association with $\mathrm{H}$. pylori infection in gastric cancer and peptic ulcer disease}

\section{S K Shukla, ${ }^{1} K N$ Prasad, ${ }^{1}$ A Saxena, ${ }^{1} U$ C Ghoshal, ${ }^{2}$ $N$ Krishnani, ${ }^{3} N$ Husain. ${ }^{4}$ \\ ${ }^{1}$ Departments of Microbiology, ${ }^{2}$ Gastroenterology, ${ }^{3}$ Pathology, Sanjay Gandhi Postgraduate Institute of Medical Sciences, Lucknow 226 014, India, ${ }^{4}$ Department of Pathology, Chha- trapati Shahu Ji Maharaj Medical University, Lucknow, India}

Helicobacter pylori and Epstein-Barr virus (EBV) infections are common world-wide. Though $H$. pylori infection is a major factor in gastroduodenal diseases, its role in association with EBV infection is unknown. We prospectively studied the association of $H$. pylori and EBV DNA load in patients with gastroduodenal diseases. Biopsy sample were collected from 200 adult patients [non-ulcer dyspepsia (NUD) 100, PUD 50, GC 50] undergoing upper gastrointestinal endoscopy. H. pylori infection was diagnosed by rapid urease test, culture, PCR and real-time PCR. EBV DNA was detected by non-polymorphic Epstein-Barr nuclear antigen-1 (EBNA-1) gene based Real-time PCR. In patients with GC and PUD, EBV DNA was detected more often than NUD (GC versus NUD $=90 \%$ versus $37 \%, p$ $<.001$ and PUD verses NUD 754.00 verses $86.79 p<.001$ ). The concentration of EBV DNA was significantly higher in H. pylori infected patients $(p=.015)$. The median concentrations of EBV DNA were higher in diffuse type of carcinoma than intestinal type but the difference was not significant. In conclusion, the trend of EBV DNA load was higher in $H$. pylori positive individuals suggesting the probable role of $H$. pylori in modulating the conversion of EBV to its lytic phase.

\section{STH13}

Prevalance of $\boldsymbol{H}$. pylori infection in patients undergoing upper g.i.endoscopy and relationship of $\boldsymbol{H}$. pylori infection with common upper GI disorders

M H Raza, Shadma Wahab, Faroze Ahmad Khan.

Department of Surgery J N Medical College, Aligarh Muslim University, Aligarh 202 002, U P

Aim: to study the prevalance of H.pylori infection in patients undergoing upper GI endoscopy. Relationship of H.pylori with upper GI disorders.

Methods: One hundred and forty-three patients underwent upper GI endoscopy and were tested for H.pylori by various tests.

Results: Out of 143 patients 108 tested positive for $H$. pylori. The overall prevalance was $75.5 \%$.

Conclusion: As compared to those having normal endoscopic study, the H.pylori were significantly positive in various disease states such as duodenitis $(100 \%)$, gastritis $(90.3 \%)$, peptic ulcer disease $(91.6 \%)$.

\section{STH14}

Analysis of alpha 1 antitrypsin activity (AAT) and ceruloplasmin $(\mathrm{CP})$ levels in peptic ulcer: A comparative study

B Mahabubunnisa, Aleem A Khan, M Aejaz Habeeb, C M Habibullah.

Centre for Liver Research and Diagnostics, Kanchanbagh, Hyderabad 500 058, AP, India

Introduction: Peptic ulcer is a multifactorial disorder with different genetic and pathological manifestations. Genetic factors such as familial aggregation, twin studies $\mathrm{ABO}$ blood groups, $\mathrm{ABH}$ non secretor status and hyperpepsinogenemia have been reported.

Methods: Blood samples were collected from the endoscopically confirmed 56 cases of peptic ulcer and healthy controls for the analysis of AAT and CP. The levels of AAT and $\mathrm{CP}$ in serum were done by the standard kit methods.

Results: Increased levels of AAT and CP were observed in patients with increase in H.pylori infection as compared to controls.

Conclusions: Most of the patients were reported to be infected with chronic $H$. pylori which lead to increase in urease, resulting in release of free radicals. Because of the tissue damage AAT and CP levels were increased. This study 
reveals that the increase in levels of both AAT and CP is to prevent the tissue damage by scavenging the free radicals.

\section{STH15}

Initial experience with modified quadruple treatment regimen containing doxycline, levofloxacin, nitazoxanide for Helicobacter pylori eradication

Vijay Sharma, ${ }^{1, *}$ Richa Sharma, ${ }^{1}$ Puneet Rizwani, ${ }^{2}$ Vitthal Das Maheshwari ${ }^{2}$.

${ }^{1}$ Gastroenterology, ${ }^{2}$ Medicine, Mahatma Gandhi Medical College and Hospital, Jaipur, India

Introduction: Eradication rates of Helicobacter pylori have declined in recent years. Newer, more effective options are warranted.

Aims and Method: To explore effectiveness of new, low-cost quadruple modified therapy for $H$. pylori eradication. Prospective study conducted from 2009-2010. Patients with endoscopically documented peptic ulcer and erosive/ nodular antral gastritis and rapid urease test confirmed H.pylori positivity. Subjects were treated with modified AHP regimen consisting rabeprazole $20 \mathrm{mg}$ bds, doxycycline $100 \mathrm{mg} 1 \mathrm{od}$, Levofloxacin $500 \mathrm{mg} 1$ od for 10 days and nitazoxanide $500 \mathrm{mg} 1$ bds for first five days.H. pylori eradication confirmed by repeat endoscopy and antral biopsy after 6 weeks.

Results: One hundred and seventeen naive patients and 27 previous treatment failures enrolled in the study $(65 \mathrm{~F}, 72 \mathrm{M}$, age: $16-80$ years). There were 5 drop out. Helicobacter pylori eradication was achieved in $82 \%$ (112), intention-to-treat (ITT): $82.4 \%$ (95\% CI: 71-87\%) and per-protocol (PP): $84.8 \%$ (95\% CI: 75-91\%). In treatment naive patients ITT and PP eradication rates were $86.8 \%$ (95\% CI: 71-91\%), and $88.7 \%$ (95\% CI: $75-94 \%$ ) respectively. As a second line eradication achieved in ITT $77.6 \%$. Mild side effects reported by 16 patients $(11.7 \%)$ with 3 drop outs.

Conclusion: Newer low cost quadruple regimen achieved high eradication rates as first line as well as second line regimen. This combination can have added effect on concomitant parasitic and chlamydia infection, which are common in India.

\section{Small Intestine}

\section{SI1}

\section{Histopathology of non ulcer dyspepsia-a cross sectional study}

Thayumanavan, Nithya, Jegannathan, Kannan, Ramani, Hari. Department of Gastroenterology, Madurai Medical College, Madurai 600020
Background: The histopathology of the gastric mucosa in Non ulcer dyspepsia has rarely been studied and very few reports are available in India. Reports from Nigeria and Saudi arabia have shown changes in the gastric mucosa in NUD patients. We studied the pathology of the gastric mucosa in NUD patients.

Aim: To study the histopathology of the gastric mucosa in non ulcer dyspepsia.

Methods: Structural lesions were ruled out by clinical details, ultrasonogram and upper gastrointestinal endoscopy in 173 patients with non ulcer dyspepsia. Mucosal samples were obtained from the gastric fundus, body and antrum. Specimens were analyzed for histopathological changes after staining with routine hematoxylin and eosin.

Results: Out of 173 patients with NUD, 100 patients $(66 \%)$ had mucosal lesions of which 61 patients (61\%) had pan gastritis. Isolated antral gastritis was seen in 19 patients and gastritis of the body was seen in seven patients. Thirteen patients had isolated gastritis of the fundus. Four patients with antral gastritis had intestinal metaplasia, out of which two patients were in the age group 24 months) had presence of lymphoid aggregates in mucosa.

Conclusions: Non ulcer dyspepsia was associated with significant mucosal lesions including lymphoid follicles and intestinal metaplasia despite normal endoscopy; hence routine biopsy of the mucosa is needed. This may have implications for treatment

\section{SI2}

\section{Lactose intolerance in celiac disease patients from north India}

\section{S V Rana, S Sharma, S K Sinha, K Singh.}

Department of Super Specialty of Gastroenterology, Postgraduate Institute of Medical Education and Research, Chandigarh 160 012, India

Background: Celiac disease and lactose intolerance are both relatively frequent diseases. Lactose intolerance may be due to genetic predisposition or due to injury to small intestine. Untreated celiac disease produces chronic inflammation of the small intestine mucosa, injuring the cells that ordinarily produce lactase. Celiac disease is common in North India. Diarrhea and flatulence is common symptom in these patients. Lactose intolerance may be one of the reasons of diarrhea and flatulence.

Aim: This study was planned to find out the incidence of lactose intolerance in celiac disease patients and controls.

Methods: 84 adult confirmed celiac patients and 100 age and sex matched controls were enrolled. Celiac disease was confirmed by anti-tissue transglutaminase (ttgA) measured by ELISA kit. Non-invasive Lactose hydrogen breath test 
was done in all subjects after over night fast. Hydrogen concentration was measured by using SC Microlyzer from Quintron, USA.

Results: Out of 82 adult celiac patients, 40 were females and 44 were males while out of 100 controls 46 were females and 54 were males. Mean \pm S.D of mean age in male celiac patients was $35.7 \pm 17.5$ years and of females is 36.35 \pm 10.9 years. While of controls were $36.7 \pm 16.8$ years in males and $36.3 \pm 10.3$ years in females. 58 out of $84(69 \%)$ celiac patients were lactose intolerant, while in controls 43 out of $100(43 \%)$ were lactose intolerant.

Conclusion: This study suggests that lactose intolerance was significantly higher in adult celiac patients as compared to controls.

\section{SI3}

\section{Patients with irritable bowel syndrome exhale more hydrogen than healthy subject in fasting state}

\section{Sunil Kumar, Asha Misra, Uday C Ghoshal.}

Department of Gastroenterology, Sanjay Gandhi Postgraduate Institute of Medical Sciences, Raebareli Road, Lucknow 226 014, India

Background: Irritable bowel syndrome (IBS) is often associated with abdominal bloating. Bloating may result from increased amount or distribution of gas in the gut or exaggerated perception of distension. To evaluate whether patients with IBS produce more hydrogen even in fasting state, we conducted a study with the following aims, (a) to estimate fasting breath hydrogen levels among patients with IBS as compared with healthy controls (HC), (b) to study relationship between symptoms of IBS and stool frequency and fasting breath hydrogen levels.

Methods: Eighty-one patients with IBS (Rome III criteria) and $123 \mathrm{HC}$ were included. Hydrogen breath test was performed using a gas analyzer after an overnight (12-h) fast. Both patients with IBS and HC had similar preparation before breath hydrogen estimation.

Results: Of 93 patients with symptoms of functional gastrointestinal disorders, 81 (87.1\%) met Rome III criteria and $12(12.9 \%)$ were negative, hence excluded. Patients with IBS were comparable in age $(35+11.8 \mathrm{y}$ vs. $37.5+$ $13.1 \mathrm{y}, p=\mathrm{ns}$ ) and gender [male 61/81 (75.3\%) vs. 77/123 $(62.6 \%), p=0.67]$ with HC. Average fasting breath hydrogen was higher in patients with IBS as compared to HC $(10.1+6.5$ vs. $5.5+6.2 \mathrm{ppm}, p<0.0001)$. Number of stools per week correlated with average fasting breath hydrogen excretion in patients with IBS $(r=0.26, p=0.02)$.

Conclusion: Inspite of similar preparation for the test, fasting breath hydrogen was higher in patients with IBS as compared to HC. Number of stools per week correlated with fasting breath hydrogen levels among them.

\section{SI4}

Small intestinal bacterial overgrowth (SIBO) in irritable bowel syndrome (IBS): frequency and predictors

Sanjeev Sachdeva, Ajit Kumar Rawat, Ravi Sankar Reddy, A S Puri.

Department of Gastroenterology, G B Pant Hospital, New Delhi 110 002, India

Background and Objective: Small intestinal bacterial overgrowth (SIBO) has been implicated in pathogenesis of irritable bowel syndrome (IBS), but evidence is conflicting. We aimed to study the frequency and predictors of SIBO in patients with IBS.

Methods: We studied 59 consecutive patients of IBS and 37 healthy controls (HC). Evaluation for SIBO was done by glucose breath test (GBT) using $100 \mathrm{gm}$ of glucose. Persistent rise in breath hydrogen or methane $>12 \mathrm{ppm}$ above basal (at least two readings) was considered diagnostic of SIBO.

Results: Of 59 patients, 27 were diarrhea predominant (DIBS), 11 were constipation predominant (C-IBS) and 21 were mixed type (M-IBS). Median age of patients [34 (18-47) y] was comparable to controls [35 (20-48) y] $(p=0.21)$. Patients were similar to $\mathrm{HC}$ in gender distribution [male 41/59 (69.5\%) vs. $25 / 37(67.6 \%), p=0.84]$. SIBO was more frequent in patients than healthy controls $[14 / 59(23.7 \%)$ vs. $1 / 37(2.7 \%)$, $p=0.006]$. Patients with D-IBS more often had SIBO as compared to non-diarrhea predominant IBS [10/27 (37\%) vs. $4 / 32(12.5 \%), p=0.029]$. C-IBS had lowest frequency of SIBO $[1 / 11(9 \%)]$ among all IBS subgroups. Patients with history of bloating more often had SIBO as compared to those without this symptom $[11 / 23(47.8 \%)$ vs. $3 / 36(8.3 \%), p=$ 0.001]. Among IBS patients, females more often had SIBO as compared to males [8/18 (44.4\%) vs. $6 / 41(14.6 \%), p=0.014]$. Conclusions: SIBO was more frequent in patients with IBS as compared to healthy controls. D-IBS subtype, female gender and history of bloating were predictors of SIBO in patients with IBS.

\section{SI5}

Diagnostic yield of capsule endoscopy in small intestinal disease: Analysis of 48 cases

B Ravi Shankar, G R Srinivas Rao, T L V D Prasad Babu, Vamsidhar Reddy, Chandrasekhar Reddy, Jithendar Reddy, Vamsi Krishna, Ambareesh Barat.

Departments of Gastroenterology and Gastrointestinal Surgery, Yashoda Hospital, Secunderabad, AP

Aim: To evaluate the diagnostic yield of capsule endoscopy for patients of suspected small intestinal disease. 
Method: We did a retrospective analysis of 48 consecutive cases who presented to our hospital over a 5 year period, from 2005 to 2010, with suspected small intestinal disease and underwent capsule endoscopy for the same. Capsule endoscopy results were analyzed as a proportion of the lesion detection rate for each specified indication.

Results: A total of 48 patients were included. Of these, 31 were males and 17 were females. The mean age was 48.62 years. The indications for inclusion in the study included obscure gastrointestinal bleed (28), abdominal pain (10), anemia (6) and chronic diarrhea (4). Capsule endoscopy provided a definitive diagnosis in 21 of 28 patients with obscure gastrointestinal bleed (detection rate of $75 \%$ ). For the 10 patients who presented with abdominal pain, lesions were detected in $2(20 \%) .4$ out of the 6 patients with anemia had positive findings $(66 \%)$. 1 of 4 patients with chronic diarrhea had positive findings (25\%). Conclusions: Capsule endoscopy had good diagnostic yield in for obscure gastrointestinal bleed and anemia. However, it was not so helpful in other cases.

\section{SI6}

\section{A case of refractory peptic ulcer}

P Jagadesan, B Prakash, B Shankar, G Ramkumar, K Muthukumaran, T Pugazhendhi, S Jeevan Kumar.

Department of Digestive Health and Diseases, Government Peripheral Hospital, Chennai

Introduction: Gastrinoma is one among the rare causes of refractory peptic ulcer. Sixty-eight-year old male presented with c/o recurrent upper abdominal pain for 5 years duration. Pain increased with food intake burning in nature and relieved by vomiting. No h./o abdominal distention, jaundice, loss of weight and appetite. Patient was a smoker and non alcoholic. No previous $\mathrm{h} / \mathrm{o}$ abdominal surgery. Previous records showed endoscopically proven multiple duodenal ulcers with partial GOO. Treated with anti- $H$. Pylori regimen and PPIs for adequate duration. Current admission revealed normal baseline investigations. OGD done this time showed multiple active gastric and duodenal ulcers which extending upto Duodeno-Jejunal flexure. Fasting serum gastrin level estimated after 2 wks of PPI cessation showed value of $1350 \mathrm{pg} / \mathrm{ml}$ suggestive of gastrinoma. CECT abdomen showed prominent duodenal folds. Surgery was planned for the patient. As endoscopic ultrasound and somatostatin receptor scintigraphy for localization of tumor could not be done due to financial restraints, patient was put on high dose PPIs. Repeat endoscopy after 2 months showed complete healing of ulcers with symptomatic improvement. Patient was advised lifelong high dose PPIs and he is on regular follow-up.
Gastrinoma should be suspected in every case of refractory peptic ulcer. PPIs should be withheld for 2 weeks before estimation of serum gastrin levels.

\section{SI7}

An interesting case of subacute intestinal obstruction

$R$ Senthil Kumar, $T$ Arun, $R$ Balamurali, $S$ Chitra, $T$ Pugazhendhi, S Jeevan Kumar.

Department of Digestive Health and Diseases, Government Peripheral Hospital, Chennai

Introduction: Malignancy of the small intestine is rare. 53 years old male presented with complaints of upper abdominal pain of 6 months duration. Pain increased with food intake and associated with post cibal bloat. Vomiting for 20 days which contained undigested food particles. Vomitus bile stained but not blood stained. Passed stools once in 3 days. No blood in stools or malena. Significant loss of appetite and weight present. On examination patient was anemic, anicteric, no generalized lymphadenopathy. Examination of abdomen revealed no visible peristalsis. Mild tenderness in epigastric region. No organomegally and bowel sounds were present. Blood picture revealed microcytic hypochromic anemia. LFT and RFT were normal. OGD scopy revealed unhealthy mucosa with circumferential narrowing of D2 beyond ampulla with stasis esophagitis. Biopsy taken from the unhealthy mucosa showed non specific inflammation. Barium meal series revealed a dilated duodenal bulb and narrowing at the junction of D2 and D3. Barium passed beyond and rest of the bowel was normal. CECT Abdomen revealed a dilated duodenal bulb and D2 with a suspicious mass at the junction of D2 and D3. Laparotomy revealed a growth at junction of D2 and D3 with infiltration of mesocolon. Biopsy from the growth was taken and palliative duodeno jejunostomy was done. HPE report revealed well differentiated adenocarcinoma. Patient was started on palliative chemotherapy and is now follow up for 2 months.

\section{SI8}

K-RAS gene mutations in colorectal cancer: $A$ therapeutic and prognostic marker

S Siva Prasad, ${ }^{1}$ S Anuradha, ${ }^{1}$ G Venkat Rao, ${ }^{2}$ R Pradeep, ${ }^{2}$ M Sasikala, ${ }^{1}$ D Nageshwar Reddy. ${ }^{2}$

${ }^{1}$ Asian Health Care Foundation, ${ }^{2}$ Asian Institute of Gastroenterology, Somajiguda, Hyderabad 500 082, India

Introduction: The median survival of colorectal cancer patients has been increased from 12 to 20 months due to active new chemotherapeutic agents, novel targeted drugs and antibody therapy. Patients with K-RAS gene mutations 
do not show response to antibody treatment as mutations in KRAS gene cause for constitutively active signaling function leading to cell proliferation and cancer. Hence present study was aimed to analyze K-RAS gene mutations in colorectal cancer patients to understand frequency of mutations, disease prognosis and to take therapeutic decision.

Methods: The study included 56 cases clinically diagnosed for colorectal cancer and 50 normal samples. Codon 12, 13 of K-RAS gene were analyzed by nested PCR-RFLP and confirmed by direct sequencing, where as codon 61 was genotyped by direct sequencing method.

Result: Out of 56 cases 13 (23.21\%) cases showed K-RAS gene mutations. Among mutants 11 cases were heterozygous (GGT/TGT) and one was homozygous (GGT $>$ GTT) for codon 12 , where as only one case showed heterozygous for both the codons (GGT/GAT for codon 12, GGC/GTC for codon 13). The codon 61 was wild type in all the patients. There was significant difference between patient and control samples regarding codon 12. $(p=0.02, \mathrm{OR}=$ 1.2). Codon 12 variants showed severe disease prognosis compared to cases with wild genotype.

Conclusion: K-RAS is a significant molecular marker for colorectal cancer to understand disease prognosis and to enhance suitable treatment decision as patients with K-RAS gene mutations do not respond for antibody treatment which causes substantial financial burden.

\section{SI9}

\section{Silent information regulatory protein expression and Telomerase activity in gastrointestinal malignancies}

K Sandhya Rani, ${ }^{1}$ M Sasikala, ${ }^{1}$ G Radhika, ${ }^{1}$ S Siva Prasad, ${ }^{1}$ $M$ Guru Prasad, ${ }^{1}$ R M Mukherjee, Varadaraj Gokak, ${ }^{2}$ Sandeep Lakhtakia, ${ }^{2}$ Manu Tandon, ${ }^{2}$ G V Rao, ${ }^{2}$ D Nageshwar Reddy. ${ }^{2}$

${ }^{1}$ Asian Health Care Foundation, ${ }^{2}$ Asian Institute of Gastroenterology, Somajiguda, Hyderabad 500 082, India

Background: Sirtuins (Silent information regulatory protein) are NAD dependent histone deacetylases which play an important role in chromatin silencing, genomic stability, ageing and calorie restriction. Telomerase is demonstrated to be over expressed in many of the human cancers. Though the role of Sirtuin has been shown to be dual in cell survival and cell death, its significans in relation telomerase activity in Gastrointestinal malignancies has not been understood. Hence the present study aims to evaluate the level of sirtuin in relation to telomerase activity in the genesis of gastrointestinal cancers.

Methods: Patients undergoing surgeries for gastro intestinal malignancies $(n=29)$ were recruited. Paired fresh frozen tissue biopsies from colorectal (10), gastric (8), Pancreas (8), esophageal (3) carcinomas were obtained. The expres- sion of Sirtuin levels was assessed by westernblot and confirmed by immuno histochemistry. Telomerase activity was evaluated using TRAP assay.

Results: Sirtuin 1 was found to be significantly $(P<0.05)$ reduced by $\approx 39 \%$ in all the GI malignancies as compared to the paired normal tissue. Telomerase activity by TRAP showed $70 \%$ increase as estimated by number of telomeric repeats in the tumor tissues. IHC confirmed decreased Sirtuin 1 levels and increased telomerase expressions.

Conclusions: Sirtuins may have a tumor suppressor role in GI malignancies as indicated in this study.

\section{SI10}

Utility of suspected blood indicator (SBI) in localizing the site of obscure GI bleed by capsule endoscopy (CE)

Manohar Reddy, Gupta Rajesh, Nitesh Pratap, Manu Tandan, Sandeep Lakhtakia, G V Rao, D Nageshwar Reddy.

Asian Institute of Gastroenterology, Somajiguda, Hyderabad 500082.

Background: Wireless capsule endoscopy (CE) is an innovative technology used in the evaluation of obscure gastrointestinal bleeding (OGIB). As the capsule travels through the digestive system, collects in excess of 50,000 images, which must be reviewed by a gastroenterologist requiring as much as $90 \mathrm{~min}$ to review a single study. The "Suspected Blood Indicator" (SBI) is a software feature proposed to aid in the detection of lesions and speed reading times. The study elevated the sensitivity and the specificity of the SBI in patients undergoing CE for OGIB. Methods: A prospective analysis of 38 consecutive capsule studies was carried out on cases performed between 2007 and 2008. The PillCam ${ }^{\odot}$ SB capsule endoscope (Given Imaging, Yoqneam, Israel) was used in this study. The data was analysed using rapid 4 version. Demographic data, medications, comorbid conditions, gastrointestinal imaging and endoscopic studies were reviewed. The principal outcome variables were actual lesions identified by a gastroenterologist and presumed lesions identified by the SBI. Different types of abnormalities were recognised active hemorrhage, AVMs, red lesions (p1, p2, p0)

Results: A total of 38 recordings were evaluated, 4 were excluded. Sensitivity, specificity and positive and negative predicitive values of SBI were $94 \%, 9 \%, 34 \%$ and $75 \%$ respectively for $\mathrm{p} 1 / \mathrm{p} 2$ lesions in overt type, $64 \%, 9 \%, 42 \%$, $25 \%$ in occult type.

Conclusions: The performance SBI in detecting bleeding areas and red lesions in terms of sensitivity was good, specificity remained poor. SBI should be considered as a complimentary and rapid screening tool for identifying actively bleeding lesions. 


\section{SI11}

\section{Effect of zoledronic acid in patients with newly diagnosed adult celiac disease: a prospective, randomized study}

Mukul Kumar, Sanjay Bhadada, Rakesh Kochhar, Padala Ravi Kumar, Niranjan Khandelwal, Kapil Jain, K Chander, Nain, Usha Dutta, Anil Bhansali, Kartar Singh. Departments of Gastroenterology, Endocrinology and Radiodiagnosis, Postgraduate Institute of Medical Education and Research, Chandigarh 160 012, India

Objective: To study the effect of zoledronic acid on bone mineral density (BMD) in newly diagnosed adult patients with celiac disease $(\mathrm{CD})$.

Methods: In this randomized, prospective controlled trial 50 newly diagnosed adult $\mathrm{CD}$ patients were recruited between April 2008 and December, 2008. BMD was measured by Dual energy X-ray absorptiometry (DEXA) at the lumbar spine and bone mineral content (BMC) was estimated. Patients with low BMD were randomized into two groups; group A received calcium and Vitamin D along with gluten free diet, while patients in group B in addition, received zoledronic acid.Patients were followed for one year.

Results: On the 34 patients studied, BMD was low in 28 $(82 \%)$ patients. After 1 year, the T-score improved significantly in group A $(n=15)$ from $-3.3 \pm 1.2$ to $-2.1 \pm$ $1.4 p=0.028)$ and in group $\mathrm{B}(n=13)$ from $-2 \pm 1.3$ to $-1.1 \pm$ $1.8, p=0.001$. However between the two groups, the difference was not statistically significant $(p=0.087)$. The $\mathrm{BMC}$ improved from $0.66 \pm 0.16$ to $0.87 \pm 0.15 ; p=0.024$ in group A and from $0.80 \pm 0.23$ to $0.86 \pm 0.21 ; p=0.015$ in group B. However between the two arms, the difference was not statistically significant $(p=0.087)$.

Conclusions: Low BMD was seen in $82 \%$ of newly diagnosed adult $\mathrm{CD}$ patients. Zoledronic acid had little additive effect on bone BMD in newly diagnosed adult celiac patients compared to a gluten free diet with calcium and vitamin D supplementation.

\section{SI12}

\section{Clinical presentation of celiac disease among adults and children is different}

Kapin Jain, R Kochhar, B R Thapa, P Rawal, S Bhadada, K K Prasad, U Dutta, P Malhotra, C K Nain, A Bhansali, $S$ Varma, K Singh.

Departments of Gastroenterology, Endocrinology and Internal Medicine, Postgraduate Institute of Medical Education and Research, Chandigarh 160 012, India
Objectives: To compare the clinical presentation and profile of newly diagnosed pediatric and adult celiac patients.

Methods: All patients diagnosed between year 1997 and 2007 in the Pediatric GE and Adult GE clinic were retrospectively analyzed. Details of physical examination and routine laboratory investigations like hemogram, renal and liver function tests were retrieved. Diagnosis of celiac disease was based on the modified ESPGHAN criteria.

Results: A total of 434 children and 298 adults were included in the study. The mean age of diagnosis was 6.53 years $(1-11)$ in children and 29.313.3 years $(16-73)$ in adults. The mean duration of symptoms before diagnosis was 3.52.5 years in children and 4.894.6 years in adults. Diarrhea as the presenting symptom was seen in $74 \%$ of children and $58.7 \%$ of adults. Symptoms of anemia were observed in of children and of adults but $\mathrm{Hb}$ below $12 \mathrm{gm} \%$ was seen in $84 \%$ of children and $94 \%$ of adults. Growth failure /short stature was seen in $11 \%$ of children and $17.4 \%$ of adults and type 1 diabetes was seen in $0.4 \%$ of children and $7 \%$ of adults. Esophageal webs (2\%), dermatitis herpetiformis $(0.2 \%)$ and infertility/ amenorrhea (3\%) were seen in only in adults. Endoscopically grooving/ scalloping were seen in $78 \%$ of children and $70 \%$ of adults. Histologically children had more severe disease. (Marsh IIIa 13\%, Marsh IIIb 87\%) than adults, Marsh II 11\%, Marsh IIIa 29\%, Marsh IIIb 59\%).

Conclusions: Clinical profile of CD differs in children and adults.

\section{SI13}

\section{Interesting cases of subacute intestinal obstruction}

B Vinoth, Kani Shaikh Mohammed, Biggs Saravanan, M Malarvizhi, K Premkumar, K Caroline Selvi, P Ganesh, P Padmanabhan, Mohammed Ali.

Department of Medical Gastroenterology, Madras Medical College, Chennai 600003

Introduction: We are presenting three cases who presented with features of sub acute intestinal obstruction (SAIO), namely abdominal distension, abdominal pain and vomting with confirmatory findings in abdominal X-Ray. CT abdomen was taken in all patients.

Case 1: 35 year old female presented with features of SAIO. CT showed partial obstruction at mid jejunum due to bowel thickening with few lymph node enlargement. laparotomy was done and a resection and anastomosis was carried out.HPE showed granuloma with casseation. ATT was started and the patient is doing well. 
Case 2: 25 year female came with features of SAIO. Evaluation showed jejunoileal obstruction.surgery in the form of resection and anastomosis was done.HPE was suggestive of crohn's disease. She was started on PENTASA and is doing well.

Case 3: 44 year male a known case of VP shunt presented with features of SAIO. Investigations showed jejunal obstruction with adhesions at the site of VP shunt tube. Laparotomy was done and it showed adhesions at the site of the VP shunt tube (Gummy tube syndrome). Adhesions was releaved and the patient was managed with antibiotics. Conclusion: Awareness and proper investigation and prompt surgical intervention aids in releaving the obstruction and providing correct medical therapy.

\section{SI14}

\section{Bleeding caused by rapidly appearing and disappearing aneurysms}

$M$ Paliwal, $R$ Puri, $K$ Madan, $S R$ Mishra, $S$ Bhagat, $N$ Saraf, $S S$ Baijal, $R$ Jain, $R$ Sud.

Medanta Institute of Digestive and Hepato-biliary Sciences, Gurgoan, Haryana, India

Background: Abdominal aneurysms are on several occasions reported in vasculitis and can cause gastrointestinal bleed, however it is rarely documented to rapidly appear and disappear, we report one such case

Methods: A 13 year girl gave history of melena for 1 day, 2 weeks prior to presentation. She had a past history suggestive of Reynolds's phenomenon. General physical and systemic evaluation was normal, except for pallor. Gastroduodenoscopy was normal, colonoscopy revealed few rectal ulcers, biopsy was taken. She underwent CT angiography which revealed aneurysm in the proximal jeujunal arterial branch of superior mesenteric artery (SMA). She was taken up for conventional angioembolization the next day, however the aneurysm had disappeared and multiple micro aneurysms were noted in distal jejunal branches. Histopathology revealed fibrinoid necrosis in the medium sized arteries suggestive of polyarteritis. She was evaluated and met four out of 10 WHO criteria of polyateritis nodosa. Subsequently started on methyprednisolone. CTangiography prior to discharge revealed a new aneurysm in the jejunal branch of superior mesenteric artery. 2 weeks later she presented with massive hematochezia, after resuscitation she underwent conventional angiography, revealing multiple arterial microaneurysm in distal jeujenal, ileal branches of SMA and sigmoid branches of inferior mesenteric artery, not actively bleeding. She underwent laprotomy revealing multiple jejunal submucosal adenomata (5 $\mathrm{mm}$ size), which were ligated. The patient has been started on cyclophosphamide pulse therapy and presently is symptom free for last 3 months of follow up.

Conclusion: A rare cause of gastrointestinal bleeding caused by rapidly appearing and disappearing aneurysms

\section{SI15}

Spectrum of malabsorption syndrome in northern Indian adults and factors differentiating celiac disease and idiopathic malabsorption in the tropics (tropical sprue)

Uday C Ghoshal, Mansi Mehrotra, Sunil Kumar, Ujjala Ghoshal, ${ }^{*}$ Narendra Krishnani, ${ }^{* *}$ Asha Misra, Rakesh Aggarwal, Gourdas Choudhuri.

${ }^{1}$ Departments of Gastroenterology, ${ }^{*}$ Microbiology and ${ }^{* *}$ Pathology, Sanjay Gandhi Postgraduate Institute of Medical Sciences, Raebareli Road, Lucknow 226 104, India

Background: Etiology of malabsorption syndrome (MAS) differs in tropical and temperate countries over time; clinical and laboratory parameters may help differentiation between various causes.

Method: Causes of MAS and factors differentiating tropical sprue (TS) from celiac disease (CD) were determined in 275 patients.

Results: Using standard criteria, causes in 275 patients (age 37.5+13.2-y, 170, 61.5\% male) were, TS 101 (37\%), CD 53 (19\%), small intestinal bacterial overgrowth $28(10 \%)$, AIDS $15(5.4 \%)$, giardiasis $13(5 \%)$, hypogammaglobulinemia $12(4 \%)$, intestinal tuberculosis $7(2.5 \%)$, strongyloidiasis $6(2 \%)$, immunoproliferative small intestinal disease $5(2 \%)$, Crohn's disease 6 $(2 \%)$, amyloidosis $4(1.5 \%)$, intestinal lymphangiectasia 3 (1\%) and unknown $22(8 \%)$. On univariate analysis, patients with $\mathrm{CD}$ were younger than TS $(30.6+12-\mathrm{y}$ vs. $39.3+12.6-\mathrm{y}, \quad p<0.001$ ), (age <35-y: 37/53, 70\% vs. $40 / 101,40 \%, p=0.0003)$, often Punjabi $(8 / 53,15.1 \%$ vs. $3 / 101,3 \%, p=0.009)$, had lower body weight $(41.3+$ $11.8-\mathrm{kg}$ vs. $49.9+11.2-\mathrm{kg}, p<0.001)$, longer diarrhea duration (median 36-mo, inter-quartile range 17.8-120 vs. 24-mo, $8-48, p=0.01)$, less frequent stool (6/day, $5-8$ vs. $8,5-10, p=0.03)$, lower hemoglobin (9.4+ $3.2-\mathrm{g} / \mathrm{dL}$ vs. $10.4+2.7-\mathrm{g} / \mathrm{dL}, p=0.04)$, higher platelet count $(2,58,000 / \mathrm{mm} 3$, range $1,35,500-3,23,500$ vs. $1,60,000,1,26,000-2,58,000, p=0.038$ ), hepatomegaly $(9 / 53,17 \%$ vs. $4 / 101,4 \%, p=0.01)$, subtotal or partial villous atrophy $(36 / 50,72 \%$ vs. $28 / 87,32 \%, p<0.001)$. Younger age $(<35-\mathrm{y})$, longer diarrhea duration, Punjabi race, higher platelet count and villous atrophy were significant on multivariate analysis. 
Conclusions: TS and CD are common causes of MAS among Indian adults. Punjabi race, younger age $(<35-\mathrm{y})$, longer diarrhea duration, higher platelet count and villous atrophy were associated with CD.

\section{SI16}

\section{Spirus enteroscopy: Indian experience Kothari Medical Centre, Kolkata}

\section{Karmabir Chakravartty, Ujjal Kumar Bhakat.}

Department of Gastroenterology, Kothari Medical Centre, Kolkata, India

Since capsule-endoscopy came into vogue, more and more physicians became interested in the management of small bowel diseases. However, with an average length of $6.7 \mathrm{~m}$., a free mesentery resisting standard push-to-advance technique, small bowel remains most poorly suited to standard endoscopy. Double-balloonenteroscopy (DBE), is considered gold standard for investigation and therapy of small intestinal diseases. However, it is a time consuming procedure (oral route 20-95 min, Average $78 \mathrm{~min}$ ) 1. Akerman et al first introduced spiral-enteroscopy (DSB) in 20072 as rapid method of deep small-bowel intubation.

To evaluate ease, safety and efficacy of DSB we performed spiral-enteroscopy on 162 patients between 30.4.2009 to 17.7.2010 (Male-93, female-69).

Indications: Pain abdomen-53.7\%, diarrhea-19.75\%, obscure GI bleeding-17.28\%, suspected intestinal obstruction-3.7\%, Fe-deficiency anemia-2.48\%, nauseavomiting-2.48\%, and hepatico-jejunostomy stricture dilatation-0.61\%. We diagnosed Crohn's disease$37.56 \%$, Koch's- $6.24 \%$, coeliac-3.6\%, angiodysplasia$5.8 \%$, tumors (GIST, polyp, lymphoma.) $-4.4 \%$, strictures-3.1\%, diverticulum-2.4\%, non-specific jejunitis-2.2\%, worm infestations-1.8\%, NSAID ulcers$0.8 \%$, anastomotic ulcers- $0.6 \%$, diaphragm disease- $0.6 \%$, intestinal obstruction confirmed-o.6\% and obstruction overruled-3.1\%, normal study-22.9\%. Enteroscopy could not be completed in $4.3 \%$ patients who had prior abdominal surgeries and possibly adhesions. $65 \%$ patients had post procedure sore throat resolving spontaneously in $48 \mathrm{hrs}$. Two patients had intestinal perforation which could be diagnosed during procedure and were successfully treated. Spiral advancement was difficult in patients with post-operative adhesions. Average time to reach the target was $25.2 \mathrm{~min}$ and the total procedure time was $28 \mathrm{~min}$.

We conclude that per-oral spirus -enteroscopy is safe and efficient technique using less than $35 \%$ of time taken by antegrade DBE.

\section{Large Intestine}

\section{LI1}

\section{Study of extraintestinal manifestations of inflammatory bowel disease}

Thayumanavan, Jeffey George, Jegannathan, Kannan, Ramani.

Department of Gastroenterology, Madurai Medical College, Madurai, Tamil Nadu 600 020, India

Background: IBD comprises conditions characterized by a tendency for chronic or relapsing immune activation and inflammation within the gastrointestinal tract. IBD manifest mainly with intestinal manifestations as well as with extraintestinal manifestations.

Objectives: 1.To study the clinical presentation of IBD in our hospital. 2. To study the extra intestinal manifestations of IBD. 3. To compare our study with published literature.

Methods: Thirty nine IBD patients attending our OPD, who satisfied the clinical, colonoscopic and histologic criteria for IBD were included. Detailed history, clinical examination,CBC blood biochemistry,USG,,colonoscopy were done. Biopsies of the colon was done. Extent of the disease was determined by colonoscopy and was classified as distal proctitis, proctosigmoiditis, left-sided colitis and pancolitis. Severity was assessed using Truelove and Witts criteria. Cases were examined for extraintestinal manifestations.

Results: Our study has 37 cases of UC and 2 cases of CD. $\mathrm{M}: \mathrm{F}=1: 2.5$. Prevalence of disease in our study is $31-$ 40 years. $81 \%$ are severe, $16 \%$ intermediate and $2.6 \%$ classified as mild.Total colitis is in $51.3 \%$ of cases, left sided colitis in $23.1 \%$, distal proctitis in $12.8 \%$, distal proctosigmoiditis in 7.7\%. 25/39 (64\%) patients had anyone of the extraintestinal features. Fatty liver $23.1 \%$, Gall stones 10.3\%,Pauciarthralgia $25.6 \%$,Polyarthralgia 7.69\%,Low backache $25.6 \%$,Erythema nodosum 2.6\%Pyoderma gangrenosum 2.6\%,Angular stomatitis $15.38 \%$, Apthous ulcer $5.12 \%$ formed the extraintestinal manifestations.

Conclusion: UC is more prevalent than Crohn's in our study. $\mathrm{M}: \mathrm{F}=1: 2.5$. Total colitis is the predominant colonoscopy finding in our study. The majority of UC cases are having severe disease (81.1\%). Extra intestinal manifestations of IBD in our study are $64 \%$. (25patients). The most common extraintestinal manifestations are Pauciarthralgia (25.6\%) and Fatty liver $(23.1 \%)$. No cases of primary sclerosing cholangitis and uveitis are found in our study. The extraintestinal manifestations in our study are comparable to published literature worldwide. 


\section{LI2}

\section{Comparison of fecal flora between non-vegetarian and vegetarian women in Southern India}

Jayakanthan Kabeerdoss, $R$ Shobana Devi, Balakrishnan S Ramakrishna.

Christian Medical College, Vellore 632 004, Tamil Nadu, India

Aim: To examine differences in the gut flora between nonvegetarian and vegetarian women in Southern India.

Methods: We recruited 32 vegetarian and 24 non vegetarian women. Participants were excluded if they had consumed antibiotics within the last 1 month. Each participant provided a sample of freshly passed stool.DNA was extracted using stool DNA mini-kit (Qiagen) and stored at -200 C. Real time PCR was used to quantitate bacteria flora targeting $16 \mathrm{~S}$ rRNA genes. Primers were directed at amplifying $16 \mathrm{~S}$ rDNA from the following groups or genera: Clostridium coccoidesEubacterium rectale group, Clostridium leptum group, Bifidobacterium genus, Bacteroides-Prevotella-Porphyromonas group, Lactobacillus group, Faecalibacterium prausnitzii and total Bacteria. Real-time PCR was done in a Chromo4 real-time PCR detection system by using SYBR Green master mix. Comparisons between groups were done using Mann-Whitney tests and two-tailed $\mathrm{P}$ values less than $0 \bullet 05$ were taken as significant.

Results: Between vegetarian (V) and non-vegetarian (NV) women, there was no significance of age, height, weight, social economic status and BMI. Energy intake (p-0.015), calcium ( $\mathrm{p}-0.034)$ and crude fiber $(\mathrm{p}-0.015)$ were higher in NV compared to $\mathrm{V}$ group. Fecal levels of Clostridium coccoidesEubacterium rectale group lower in V group (Median 0.043) compared to NV group (median 0.103 and Interquartile range $0.05-0.22)$ ( $p$ 0.0017). There was no difference noted on other bacterial groups between the two groups.

Conclusion: This study documents an interesting association between non-vegetarian food habit and increased level of Clostridial cluster XIVa in young women in India.

\section{LI3}

\section{Pro-inflammatory and anti-inflammatory cytokines level in IBS-diarrheal patients}

\section{S V Rana, S Sharma, S K Sinha, D K Bhasin, K Singh.} Department of Super Specialty of Gastroenterology, Postgraduate Institute of Medical Education and Research, Chandigarh 160 012, India

Background: Irritable bowel syndrome (IBS) is one of the most common, poorly understood functional gastro- intestinal disorder. It is characterized by chronic abdominal pain or discomfort and altered bowel habits. Previously it was thought that IBS may be due to the result of psychological problems and stress but recent focus has been shifted towards infection.

Aim: Therefore, the present study was planned to major the levels of pro-inflammatory cytokines (TNF- $\alpha$, IL- 8 and IL6 ) and anti-inflammatory cytokine (IL-10).

Methods: levels of pro-inflammatory and anti-inflammatory cytokines were measured in 50 adults diarrheal type of IBS patients and 50 age and sex matched healthy controls, of 20-65 yr age. Levels of cytokines were measured by using ELISA method.

Results: Mean \pm SD of age in IBS patients was $35.4 \pm$ 15.5 years, while in controls $38.5 \pm 14.2$ years. Levels of pro-inflammatory cytokines i.e TNF- $\alpha(86.04 \mathrm{pg} / \mathrm{ml})$, IL$8(896.56 \mathrm{pg} / \mathrm{ml})$ and IL-6 $(45.50 \mathrm{pg} / \mathrm{ml})$ were significantly higher as compared to controls TNF- $\alpha$ (4.81 pg /ml), IL$8(393.1 \mathrm{pg} / \mathrm{ml})$, IL-6 $(8.35 \mathrm{pg} / \mathrm{ml})$ respectively. The levels of IL-10, anti-inflammatory cytokine $(7.5 \mathrm{pg} / \mathrm{ml})$ was significantly lower as compared to controls $(9.65 \mathrm{pg} / \mathrm{ml})$.

Conclusion: This study indicates that pro-inflammatory cytokines were increased while anti-inflammatory cytokines were decrease in diarrheal type of IBS.

\section{LI4}

\section{Genotyping of $E$ coli strains isolated from patients} of ulcerative colitis

Chamandeep, Sunny Garg, P Behera, SK Shukla, G Nath, $V K$ Dixit, A K Jain.

Departments of Gastroenterology and Microbiology, Institute of Medical Sciences, Banaras Hindu University, Varanasi 221 005, U P, India

This study was done to know the frequency of intestinal pathogens in well established cases of ulcerative colitis on maintenance therapy, presenting with relapse. We further extended our studies to molecular analysis of E Coli strains isolated from the rectal mucosal tissues in ulcerative colitis patients and compared to those isolated from non-specific diarrhea cases used as controls. Stool samples were taken from 44 cases and 20 controls. It was seen that E Coli was the most common (45\%) followed by Proteus, Pseudomonas, Enterococcus, Shigella, Entemoeba, Candida and Ascaris (one each). Later rectal mucosal biopsy was obtained from all the 64 patients (44 cases and 20 controls) and subjected to ERIC-PCR analysis. 1-9 bands per isolated per reaction produced and band sized ranged from $200 \mathrm{bp}$ to $3-5 \mathrm{kbp}$. ERIC-PCR analysis had a discriminatory index (DI) of 0.845 . Dendrogram was constructed that showed 3 major clusters at 
dissimilarity coefficient of 3 . The largest cluster comprised of 42 strains (cluster 1), out of which 39 were from UC patients. Another cluster comprised of 18 strains (cluster 2), out of which 17 were from nonspecific diarrhea patients and cluster 3 comprised of 4 strains, all from UC patients. So, E Coli isolated from UC patients formed a different cluster from non-specific diarrhea patients on dendrogram.

\section{LI5}

\section{Utility of Mycobacterial DNA detection using polymerase chain reaction in diagnosis of abdominal tuberculosis}

Mansi Mehrotra, Ujjala Ghoshal, ${ }^{*}$ Uday C Ghoshal. Departments of Gastroenterology and *Microbiology, Sanjay Gandhi Postgraduate Institute of Medical Sciences, Raebareli Road, Lucknow 226 014, India

Background: Abdominal tuberculosis is difficult to diagnose as acid fast bacillus (AFB) is not detected in all patients and culture is time-consuming. We studied utility of nested polymerase chain reaction (PCR) in its diagnosis.

Method: Patients with suspected abdominal tuberculosis underwent cytopathological and radiological investigations and tests to show AFB on smear, culture and PCR on colonoscopic biopsy and ascitic fluid. Patients with AFB on either smear or culture or those with suggestive endoscopic and radiological features and response to anti-tubercular treatment were diagnosed abdominal tuberculosis.

Result: Of 53 patients (age 38.2 $\pm 14.4-\mathrm{y} ; 27,51 \%$ female) screened, the diagnosis of tuberculosis was confirmed in 31 (27 intestinal and 4 peritoneal), 4 had other diseases (adenocarcinoma cecum in two, AIDS and Crohn disease one each) and 18 remained undiagnosed. Of 31 patients with tuberculosis, 9 (29\%) had AFB on microscopy, 7 $(22.5 \%)$ on culture, 3 on smear and PCR, 3 on culture and PCR, 1 on all three tests and $8(26 \%)$ had mycobacterial DNA on PCR though smear and culture were negative. $8 / 27$ (29.6\%) had granuloma on histology. All patients responded to treatment for tuberculosis. Mycobacterial DNA was detected in 2 patients with adenocarcinoma cecum and in none in unclassified group. PCR detected Mycobacterial DNA in 15/31 with tuberculosis and 2/22 without tuberculosis $(p=0.002)$. Sensitivity, specificity, positive predictive and negative predictive values of PCR in diagnosis of abdominal tuberculosis were $48 \%, 48 \%$, $88 \%$ and $55 \%$, respectively.

Conclusion: Detection of Mycobacterial DNA on PCR is useful in diagnosis of abdominal tuberculosis.

\section{LI6}

Water infusion versus air insufflation colonoscopy in unsedated patients using indegineously developed water pump-impact on patient discomfort

Amol Samarth, C Vashishtha, Prabha Sawant, Jignesh Patel, A Agasti, M Nasa.

Department of Gastroenterology, L T M M C and L T M G H, Sion, Mumbai 400 022, India

Introduction: We studied impact on patient discomfort of exclusive water infusion versus air insufflation colonoscopy in unsedated patients using an indigeneously developed water pump.

Method: Consecutive 88 patients posted for colonoscopy (September 2009 to November 2009) were randomized to undergo blinded water infusion (group-A -44 patients) or air insufflation (group-B- 44 patients) colonoscopy. Pain was recorded using visual-analog-scale on level 0 to 5 with 0 being no pain, 1 being mild and 5 being maximum tolerable pain. Air insufflation was used during withdrawl. A modified submersible water pump instilled water through biopsy channel.

Observations: Mean age was comparable in both groups ( $34 \pm 20.14$ years group-A, $32 \pm 18.15$ group-B). M:F ratio in group-A was 1.31: 1 and $1.58: 1$ in group-B. Cecal intubation was achieved in $93.18 \%$ patients (41 of 44 ) in group-A as compared to $88.63 \%$ patients (39 of 44 ) in group-B $(p=0.07684)$. Maximal patient discomfort at any time during the insertion (2 / 5 VAS) was significantly lower in group-A $(29.54 \%)$ as compared to group-B $(65.9 \%)(p<0.01)$. Pain at first bend of sigmoid colon and splenic flexure was significantly low in group-A $(p<0.05)$. Time to cecal intubation was comparable; $19.26 \pm 6.92 \mathrm{~min}$ in group-A versus $18.66 \pm 4.80 \mathrm{~min}$ in group-B (p-NS).

Results: Water infusion colonoscopy was associated with highly significantly lower all procedure maximal pain as well as pain at first bend of sigmoid colon and splenic flexure. Time to cecal intubation and withdrawal times were comparable in both groups.

\section{LI7}

Clinical evalution of efficacy and safty of curcumin enema as an induction therapy for ulcerative colitis - A prospective study

A K Agasti, P Jignesh, M Nasa, $R$ Goyal, P Patki, (a) Prabha Sawant.

Department of Gastroenterology, Lokmanya Tilak Muncipal Medical College and General Hospital, Sion, Mumbai 400 022.@Head, Clinical Trials and Medical Service, Himalaya Company, Bangalore, India 
Aim: To study the efficacy and safty of curcumin enema as an induction therapy in patient with proctitis and proctosigmoiditis.

Methods: 30 patients in a span of 3 months having distal UC were included in study. All patients were in remisson and later developed exacerbation of symptoms. All had undergone proctosigmoidscopy and UCDAI (Ulcerative Colitis Disease Activity Index) was calculated. 20 patients whose UCDAI $>7$ and $<10$ were included in study. All patients were allowed to continue their current medication and advised curcumin enema $(20 \mathrm{ml})$ once daily. All were followed at 4 and 8 weeks interval and UCDAI was calculated at each follow up. Clinical response was defined by a decrease UCDAI 3 points from patients baseline score and remisson, UCDAI 2 or less as per standard definition.

Results: At study initiation out of 20 patients 10 had 7, 5 had 9 and 8 UCDAI respectively. At 4 weeks $12(60 \%)$ patients achieved clinical response and at 8 weeks all $(100 \%)$ patients achieved clinical response. At 4 weeks none had clinical remission but $4(20 \%)$ developed clinical remission at 8 weeks. No patients developed any side effects to curcumin enema during therapy and compliance was good.

Conclusion: Curcumin enema definitely achieves an excellent clinical response rate, it is safe and can be used as an adjunct to treatment in patients with acute exacerbation of distal ulcerative colitis,But our clinical remission rate was less, patients may require a higher dose for achieving a clinical remission.

\section{LI8}

\section{Profile of ulcerative colitis in a tertiary centre in northeastern part of India}

Pratap Behera, V K Dixit, S K Shukla, A K Jain. Department of Gastroenterology, Institute of Medical Sciences, Banaras Hindu University, Varanasi 221005 , UP, India

Background: Ulcerative colitis has worldwide occurrence. Earlier considered to be common in Europe and North America, now it has been seen frequently in India too.

Aim: To study clinical profile, natural history and course of ulcerative colitis patients in a tertiary centre in north eastern part of India.

Methods: Naive patients of Ulcerative colitis and previously diagnosed cases presenting with relapse during January 2009 to June 2010 were included in study. The course of disease and response to treatment was monitored prospectively by regular follow up.

Results: Out of 142 registered patients 128 could be followed up. Among which 37 (28.9\%) were female and
$91(71.1 \%)$ were male. Mean age of presentation was $39 \mathrm{yrs}$ for females and $37 \mathrm{yrs}$ for males. 81 (63.28\%) were between 18-40 years. Age distribution was from 3 yrs to 70 yrs. 41 (32.03\%) had E1, $54(42.18 \%)$ had E2 and 33 $(25.78 \%)$ had E3 disease as per montreal classification. 78 (61\%) presented as severe disease, $32(25 \%)$ as moderate and $18(14 \%)$ with mild disease.5 (3.9\%) were steroid resistant, 2 of which responded to cyclosporine and 3 (2.34\%)patients underwent surgery. 30 patients $(23.43 \%)$ were steroid dependant. None had family history of UC in our study. $9(7.03 \%)$ patients had disease for more than 10 years. None of them had severe displasia or malignant change in surveillance. Extra intestinal manifestations like arthritis and arthralgia were observed in $5(3.9 \%)$ patients. Toxic megacolon was not seen.

Conclusion: Like west majority present as severe disease. Complications, extraintestinal manifestations and malignant change are relatively less common.

\section{LI9}

\section{Anti nuclear antibodies in steroid dependent ulcerative colitis}

Pratap Behera, V K Dixit, S K Shukla, A K Jain.

Department of Gastroenterology, Institute of Medical Sciences, Banaras Hindu University, Varanasi 221 005, UP, India

Background: Steroid dependence is not uncommon in ulcerative colitis (UC). Few studies have explored antinuclear antibody prevalence in UC and its association with steroid dependence in western population.

Aim: Study of steroid dependence and to determine prevalence of ANA and its association with steroid dependence in ulcerative colitis at a tertiary centre in north eastern part of India.

Methods: In prospective design consecutive patients with clinical, endoscopic and histopathologic diagnosis of UC were recruited from January 2009 to June 2010. Steroid dependence was defined as per ECCO guidelines. Patients were subjected to ANA, ANTIds DNA tests by Elisa method. Those positive for ANTI ds DNA were excluded from study.

Results: Out of 128 patients followed up, 30 (23.43\%) were steroid dependent.21 (70\%) were male and $9(30 \%)$ were female. Mean age for male was 31 years and female was 35 years in steroid dependant patients. All were presented with severe disease. $50 \%$ presented with E2 disease, $40 \%$ with E3 disease and $10 \%$ with E1 disease according to Montreal classification. Total 59 patients could be subjected to ANA and antids DNA studies. 3 patients were excluded from study because of concomi- 
tant ANTIdsDNA positivity along with ANA positivity. Out of 56 patients 19 were steroid dependent and 37 were steroid nondependent. ANA positivity was seen in 1 $(5.26 \%)$ steroid dependant and $3(8.10 \%)$ steroid non dependant patients.

Conclusion: The proportion of steroid dependence corresponds to western population but prevalence of ANA positivity and its association with steroid dependence is low in Indian population.

\section{LI10}

\section{Crohns disease manifesting as colovesical fistula}

B Ravi Shankar, C Mallikarjun, Suri Babu, G R Srinivas Rao, Vishnu Reddy, Bhargav, Jithender Reddy, Vamsi Krishna, Mohan Rao, Ambareesh Barat.

Departments of Gastroenterology and Urology, Yashoda Hospital, Secunderabad, AP, India

Presentation: A 44 year old woman presented with increased frequency of micturition, pneumaturia, faecaluria and low grade fever of 3 weeks. She had diarrhea of 10 years duration.

Investigations: The investigations revealed anemia, urinary tract infection (E. coli), colonic thickening on CT and rectal pacification during cystogram. Cystoscopy showed an opening in the trigonal area. Colonoscopy revealed nodularity, narrowing and ulceration of sigmoid colon with rectal sparing. Sigmoid colon biopsy showed dense inflammation. Colonic tissue for AFB and TB PCR was negative. Blood for gamma interferon was negative and serum ASCA IgA and IgG was positive. In view of long standing colonic symptoms, typical colonoscopic findings, presence of colovesical fistula, presence of ASCA and absence of evidence of tuberculosis - Crohn's disease was diagnosed. She was treated with 3 doses of infliximab $5 \mathrm{mg} / \mathrm{kg}$ and had a good response with cessation of symptoms. The rarity in this case is the site of fistula in Crohn's disease and it's successful management with infliximab.

\section{LI11}

\section{An unusual case of hematochezia}

T Arun, $R$ Senthil Kumar, $R$ Balamurali, $S$ Chitra, T Pugazhendhi, S Jeevan Kumar.

Department of Digestive Health and Diseases, Government Peripheral Hospital, Chennai 600 010, India

Introduction: Tuberculosis is common in India. However rectal involvement is very rare.
Case Report: A 14-year-old female patient came with abdominal pain and distension for one month associated with low grade fever and bleeding per rectum for 20 days. She had decreased appetite and loss of weight. No history of contact with TB. Secondary amenorrhea was present for the past 4 months. Systemic examination was normal except for hepatomegaly and ascites. Per rectal examination revealed nodular mass felt in the tip of the finger and finger stained with blood. Complete blood counts, Renal and Liver function tests normal. Viral markers negative. USG abdomen: Hepatomegaly with normal echoes. Ascites present, spleen normal, portal vein measured $10 \mathrm{~mm}$. Ascitic fluid analysis showed low SAAG, high protein. Ascitic fluid Adenosine deaminase level was $120 \mathrm{IU} / \mathrm{l}$. Mantoux was strongly positive [15 mm]. CXR showed right upper zone infiltrates. Sputum AFB was positive. UGI scopy was Normal. Colonoscopy revealed a circumferential, ulcero nodular lesion at 5 from anal verge which extended to $15 \mathrm{cms}$. Biopsy showed fragments of rectal mucosa with ulceration. Lamina propria showed collection of epitheloid cells, few giant cells,sheets of acute and chronic inflammatory cells with fibroblasts, suggestive of tuberculosis. Patient was started on category I anti tuberculous regimen and responded well to treatment.

Discussion: Rectal tuberculosis with sputum positive pulmonary tuberculosis and tuberculous ascites is very rarely reported in literature. Rectal tuberculosis should be differentiated from crohns disease and malignancy.

\section{LI12}

\section{Association of genetic variants of CD14 gene and its expression in South Indian ulcerative colitis patients}

G Sivaram, Santosh K Tiwari, Avinash Bardia, G Manoj, $R$ Saikant, Md. Aejaz Habeeb, Aleem A Khan, C M Habibullah.

Centre for Liver Research and Diagnostics, Deccan College of Medical Sciences, Kanchanbagh, Hyderabad 500 058, India

Background: Ulcerative colitis (UC) is characterized by chronic inflammation of colon and rectum attributable to an exaggerated immune response. The etiology remains highly elusive with several factors involved in the pathogenesis of IBD. CD14 also known as lipopolysaccharide (LPS) receptor is a single-copy gene encoding 2 protein forms. The CD14 C-T polymorphism at promoter region was detected and found to regulate the density of CD14 expression on monocytes, activation of monocyte to secrete inflammatory cytokines by lipopolysaccharide and raised levels of sCD14 in serum and to be associated with various diseased conditions. Therefore the present study was 
designed to investigate the association between genetic polymorphism in the $\mathrm{CD} 14$ gene in correlation with $\mathrm{CD} 14$ expression in the pathogenesis of UC.

Methods: CD14 genotyping was performed using refractory mutation system-polymerase chain reaction (ARMS-PCR) in 189 unrelated patients (age range: $18-80$ years; $86-\mathrm{UC}$, 103- healthy controls) and CD14 expression levels were measured using flowcytometer using fluorescent antibodies tagged to MNC isolates from freshly collected whole blood. Results: CD14 genotype and allele frequencies were calculated in UC and healthy controls. The frequency of genotype mutant T-allele was significantly higher in patients with UC than the controls $(0.325(n=67)$ vs. $0.454(n=80))$ p 0.009 . The subjects with $\mathrm{C} / \mathrm{T}$ (heterozygous) and T/T (homozygous) carriers at the 159 codon had high CD14 expression levels compared to healthy controls. Conclusion: Our results suggest that polymorphisms in the CD14-159C/T variants with over expression of CD14 play an important role in the pathogenesis of UC.

\section{LI13}

\section{Genetic polymorphisms in base-excision repair pathway} genes and risk of ulcerative colitis

Avinash Bardia, Santosh K Tiwari, G Sivaram, R Saikant, $G$ Manoj, Md. Aejaz Habeeb, Aleem A Khan, C M Habibullah.

Centre for Liver Research and Diagnostics, Deccan College of Medical Sciences, Kanchanbagh, Hyderabad 500 058, Andhra Pradesh, India

Background: Ulcerative colitis (UC) is a disease of the 20th century and is associated with the rise of modern, westernized industrial society. Although the cause remain incompletely understood, a multitude of etiological hypotheses have been put forward which ranges from infective etiology to environmental and genetic influences. Base excision repair (BER) is a very important mechanism for repairing DNA damage. Apurinic/apyrimidinic endonuclease 1 (APE1) and X-ray cross-complementing group 1 (XRCC1) are DNA repair proteins that play important roles in the base excision repair pathway. Polymorphisms in these genes result in altered DNA repair capacity and thus may impact UC. Therefore the present study was designed to examine the polymorphisms associated with DNA repair genes, namely: XRCC1 Arg399Gln, APE1 Glu148Asp and investigate their role as susceptibility markers for ulcerative colitis.

Methods: $3 \mathrm{~mL}$ venous blood sample from 235 unrelated patients (age range: 18-80 years; 107- UC, 128- healthy controls) were collected from patients undergoing colonoscopy. Genomic DNA was isolated and genotyped for
XRCC1 Arg399Gln and APE1 Glu148Asp polymorphic sites by Refractory mutation system-polymerase chain reaction (ARMS-PCR).

Results: The frequency of genotype G/A (heterozygous), A/ A (homozygous mutant) at codon 399 of XRCC1 gene and $\mathrm{T} / \mathrm{G}$ (heterozygous), G/G (homozygous mutant) at codon 148 of APE1 gene were significantly higher in patients with UC than the controls $(68.22 \%$ and $10.28 \%)$ and $(64.48 \%$ and $13.08 \%$ ).

Conclusion: The result suggests that variants of XRCC 1 and APE1 genes increase the risk of UC and also suggests that polymorphisms in these genes play an important role in the pathogenesis of UC.

\section{LI14}

\section{Effect of methanogenic flora on lactose intolerance in ulcerative colitis patients}

S V Rana, R P Ola, J Kaur, V P Singh, S K Sinha, $D K$ Bhasin, $K$ Singh.

Department of Gastroenterology, Postgraduate Institute of Medical Education and Research, Chandigarh 160 012, India

Introduction: Ulcerative colitis (UC) is a chronic inflammatory condition that is related to the gastrointestinal tract with an unknown aetiology. The role of lactose malabsorption in ulcerative colitis is controversial. Higher percentage of lactose intolerance has been reported in UC patients measuring only hydrogen concentration, which may be higher if methane is measured along with hydrogen.

Aim: Therefore, this study was planned to find out the incidence of lactose intolerance by measuring methane and hydrogen in UC patients.

Methods: This study was conducted prospectively and included 30 patients with UC and 30 age and gender matched apparently healthy controls. Each subject underwent a breath test using $25 \mathrm{~g}$ lactose after an overnight fast. An SC Microlyser from Quintron, USA, was used to measure methane and hydrogen at baseline and at every $30 \mathrm{~min}$ for $4 \mathrm{~h}$. Lactose intolerance was defined as rise $>20 \mathrm{ppm}$ over baseline value of hydrogen/methane or both in two consecutive readings within $4 \mathrm{~h}$.

Results: UC and control groups included $69.9 \%$ and $66.6 \%$ men, respectively. Mean age in two groups was $45.5 \pm$ 20.6 years (range $25-68$ years) and $46.6 \pm 22.5$ years (range 24-68 years), respectively. Incidence of lactose intolerance in UC patients was $63.3 \%(19 / 30)$ while in controls $40 \%$ $(12 / 30)$. The difference was significant $(P<0.05)$. Methanogenic flora was present in $26.7 \%(8 / 30)$ controls and only in $3.3 \%(1 / 30)$ patients of UC. 
Conclusion: Lactose intolerance was significantly higher in ulcerative colitis patients while methanogenic flora significantly less as compared to apparently healthy controls.

\section{LI15}

\section{Rectal ameboma masquerading as anorectal growth}

S Mazumder, S Khanna, D Shukla, $R$ Kapil, $R N$ Jha, A K Seth.

Dr B L Kapur Memorial Hospital, New Delhi

Background: Ameboma is a localized proliferative fibrotic thickening of the intestinal wall caused by Entamoeba histolytica. Most amebomas are reported in the right colon and present as a right iliac fossa mass. We report a rectal ameboma. Case Report: 85 years female presented with bleeding per rectum mixed with well formed stool 4 to 5 times a day for 2 days. There was no of history fever, pain abdomen or tenesmus. On examination she was pale with pulse 88 per minute and BP 140/80 mm of Hg. Systemic examination was normal. On per-rectal examination a large, firm mass was felt starting from the anal verge and there was fresh blood on the finger. Colonoscpy revealed a large polypoid lesion with nodularity and ulceration involving more than $70 \%$ of circumference of distal $5 \mathrm{~cm}$ of rectum. Also noted were multiple 1 to $3 \mathrm{~cm}$ ulcers with flat edge and yellowish slough at the base in ascending colon and caecum. The ileo-cecal valve and terminal ileum were normal. Biopsy from rectum and ascending colon showed ulceration with marked polymorphonuclear infiltration and plenty of amoebic trophozoites. She received tablet Metronidazole $400 \mathrm{mg}$ thrice daily for 10 days. Repeat procto-sigmoidoscopy done two weeks later showed complete regression of the rectal amoeboma. Conclusion: Amoeboma should be considered in the differential diagnosis of colonic growths in the tropics, irrespective of the site. Associated ulcers in right colon point to the diagnosis.

\section{LI16}

Association of genetic polymorphism in the Mannan binding lectin-2 and sera MBL levels in patients with inflammatory bowel disease

G Sivaram G, Santosh K Tiwari, G Manoj, Avinash Bardia, R Saikant, Md. Aejaz Habeeb, Aleem A Khan, C M Habibullah. Centre for Liver Research and Diagnostics, Deccan College of Medical Sciences, Kanchanbagh, Hyderabad 500 058, Andhra Pradesh, India

Background: Ulcerative colitis and Crohn's disease are the two major forms of inflammatory bowel diseases. Series of reports have hypothesized interplay of genetic and envi- ronmental factors in the pathogenesis of IBD. Polymorphism in the Mannan-binding lectin (MBL-2) gene is known to affect the structural assembly and function thereby predisposing subjects to various diseases. Therefore the present study was designed to evaluate the association between polymorphism in the MBL-2 gene with MBL levels and function in IBD patients.

Methods: $3 \mathrm{~mL}$ venous blood samples from 155 unrelated patients (age range: 18-80 years; 52- UC, 11- CD, 92- healthy controls) were collected from patients undergoing colonoscopy. Genomic DNA was isolated and genotyped for different polymorphic sites by Refractory mutation system-polymerase chain reaction (ARMS-PCR), PCR-RFLP and serum MBL concentration levels were measured using ELISA.

Results: MBL-2 genotype and allele frequencies were calculated in IBD and healthy controls. The frequency of genotype G/A (heterozygous), A/A (homozygous) at codon 54 was significantly higher in patients with IBD than the controls $(52.37 \%$ and $32.6 \%)$. The IBD patients with $54 \mathrm{G} /$ A, 54A/A carriers at the 54 codon had low serum concentrations of MBL compared to healthy controls.

Conclusion: Our results suggest that polymorphisms in the MBL-2 play an important role in the pathogenesis of inflammatory bowel disease. The result also suggests that codon 54 variants of MBL-2 gene increase the risk of IBD.

\section{LI17}

\section{A study of stool parasites, stool toxin ELISA for Clostridium difficile and colonic histopathology for cytomegalovirus in patients of ulcerative colitis and its correlation with disease activity}

H Iyer Venkatakrishnan, A B Pulimood, A Sitara Swarna Rao, B S Ramakrishna.

Department of GI Sciences, Christian Medical College, Vellore 632 004, Tamil Nadu, India

Background: Ulcerative colitis (UC) is a chronic, remittingrelapsing type of disease. In a tropical country like India, the incidence of infective colitis is very high. Treatment of these infections may prevent unnecessary immunomodulator use in subset of UC patients.

Aim: To study stool parasites, stool toxin for Clostridium difficile ELISA and colonic tissue for cytomegalovirus in patients in UC and correlate it with disease activity.

Methods: We prospectively enrolled cases of UC and after history and clinical examination, evaluated each patient for presence of stool parasites (with routine and special stains), stool toxin for Clostridium difficile and obtained segmental biopsies at colonoscopy to look for cytopathic changes of cytomegalovirus infection. Truelove-Witts criteria was used to assess disease severity. 
Results: In our ongoing study till date, we enrolled 38 patients (females $=18$, males $=20$ ). Mean age was 40.44 (range 22-71). Among the 38, 13 had mild disease, 4 had moderate disease and 21 had severe disease. 2 patients had parasites in stool (one had strongyloides larvae, one had giardia cysts) and 2 had evidence of CMV on histology. None had Clostridium difficile toxin positivity. All the patients who had parasites or CMV, had severe disease. A Fisher exact chi-square test revealed that there was no statistically significant difference in parasites /CMV in severe or non-severe disease ( $p$ value $=0.54)$.

Conclusions: Given the high prevalence in the background population, we did not find a high frequency of parasites in our patients. In those whom we found parasites or histological evidence of CMV, the disease was severe.

\section{LI18}

\section{Utility of endoscopy in evaluating abnormal findings} of the gastrointestinal tract on CT scan

\section{K Bala Murali Krishna, B Prabhakar, B Ramesh Kumar.} Department of Gastroenterology, Osmania Medical College, Hyderabad, India

Objective: To determine the clinical significance of incidental GI tract abnormalities identified on CT.

Methods: A retrospective study identified patients undergoing endoscopic evaluation for luminal GI tract abnormalities detected on CT between September 2009 and May 2010.

Results: 46 patients underwent endoscopy specifically for GI tract abnormalities noted on CT. CT findings included thickened appearance of the bowel wall, mass lesions and stenoses. Esophagogastroduodenoscopy (19/46 patients) gender (M/F) 11/8 abnormal findings a) distal esophagus 6/9 (66.6\%) (reflux esophagitis-3,varices-1 polyp-1, hiatal hernia-1) b) stomach 5/8 (62.5\%) (gastritis 2, ulcer 2, carcinoma-1) c) duodenum 1/2 (50\%) (adenoma-1) colonoscopy (27/46 patients) gender (M/F) 18/9 abnormal findings a) terminal ileum $1 / 2(50 \%)$ (Ileitis-1) b) right colon 4/9 (44.4\%) (malignancy-3, colitis-1) c) proximal left colon $2 / 6$ (33.3\%) (adenoma-2) d) rectosigmoid 7/10 (70\%) (malignancy-4, colitis-2, adenoma-1) CT scan changes were noted less commonly in the upper GI tract $(41.3 \%)$ than in the lower GI tract $(58.7 \%)$. The positive predictive value of abnormality on CT for upper GI tract is $63.1 \%$ whereas that for lower GIT is $51.8 \%$.

Conclusion: In our study, the highest correlation between CT findings and endoscopic abnormalities occurred in recto sigmoid followed by distal esophagus. The least correlation seen in proximal left colon. Endoscopy remains the primary modality to investigate radiologic abnormalities of the GI tract.

\section{GI Motility}

\section{GIM1}

\section{World Gasroenterology Organization Questionaire vs. Rome III Criteria}

J Kayalvizhi, Kani Shaikh Mohammed, B Vinoth, Biggs Saravanan, M Malarvizhi, K Premkumar, K Caroline Selvi, P Ganesh, P Padmanabhan, Mohammed Ali. Department of General Medicine, Madras Medical College, Chennai 600 003, India

Background: We evaluated the validity of the recently proposed questionnaire by the World Gastroenterology Organization in diagnosing Irritable Bowel Syndrome, addressing it's usefulness in delineating IBS from IBD, Colonic cancer when compared with the existing Rome III criteria.

Methods: A total of 52 (18 yrs to $63 \mathrm{yrs}$ ) patients attending Medical / GE departments who fitted into the ROME III criteria participated in the study. Patient's scores on the questionnaire were taken. And they underwent clinical examination, routine biochemical tests and colonoscopy. Results: We compared the sensitivity, specificity, predictive values of the questionnaire against those of the Rome III and found it to be much higher for the questionnaire especially the specificity (questionnaire $=93 \%$, Rome III $=14 \%$ ).

Conclusions: Our comprehensive study revealed the definite usefulness of the questionnaire in identifying patients with IBS. Because of the high specificity, the need for invasive procedures becomes unwanted without the disadvantage of missing serious pathology. Thereby it is recommendable in patients with bowel disturbance of all age group.

\section{GIM2}

\section{Psychiatric and somatic co-morbidities in Indian patients with irritable bowel syndrome at a tertiary care center}

Prashant Singh, Abhishek Agnihotri, Asef Shirazi, Rajeew P Tiwari, R Sagar, Govind K Makharia.

Department of Gastroenterology and Human Nutrition, and Psychiatry, All India Institute of Medical Sciences, New Delhi 110 029, India

Introduction: Fifty to $90 \%$ of patients with IBS have associated psychiatric illnesses in West. Somatic comorbidities are present in up to $70 \%$ of IBS patients.

Methods: In this prospective study at a tertiary care center, diagnosis along with sub-classification of IBS and associated other functional gastrointestinal disorders was made on 
the basis of Rome-III criteria. Psychiatric evaluation was done using Patient Heath Questionnaire.

Results: Of 102 patients with IBS (IBS-C 32, IBS-D 40, IBS$M$ 30), the associated other functional gastrointestinal disorders in them were: postprandial distress syndrome 45 (44.1\%), unspecified excessive belching 60 (58.2\%), cyclic vomiting syndrome $15(14.7 \%)$, and proctalgia fugax in 12 (11.8\%). 88 patients $(86.3 \%)$ had at least one somatic co morbidity. Eighty patients (78.4\%) had at least one or more psychiatric disorder. Somatoform disorder was present in 50 (49\%), major depression syndrome 43 (42.2\%), other depression syndromes 19 (18.6\%), panic syndrome 39 (38.2\%), other anxiety syndrome 27 (26.5\%), binge eating disorder $2(1.9 \%)$ and alcohol abuse in patients $2(1.9 \%)$. Only 7 patients $(6.9 \%)$ were receiving psychiatric treatment. The associated somatic co-morbidities in them were low back pain in $41(40.2 \%)$, fibromyalgia in $33(32.3 \%)$, tension headache in 34 (33.3\%), migraine 22 (21.6\%), cluster headache 4 (3.9\%), temporomandibular joint disorder in $4(3.9 \%)$, sexual dysfunction in $33(32.3 \%)$, premenstrual syndrome in 11 (37.5\% of premenopausal females), dysmenorrhea in 13 (54.2\% of premenopausal females) and chronic fatigue syndrome in $46(45.1 \%)$. Conclusion: Majority of patients with IBS presenting to a tertiary care center have associated psychiatric, and somatic co-morbidities and only a very few of them have received specific psychiatric evaluation and treatment.

\section{GIM3}

\section{Correlation between severity of irritable bowel syndrome and quality of life}

\section{Abhishek Agnihotri, Prashant Singh, V Srinivas, Rajesh Sagar, Govind K Makharia. \\ Departments of Gastroenterology and Human Nutrition, Biostatistics and Psychiatry, All India Institute of Medical Sciences, New Delhi 110 029, India}

Introduction: A correlation between degree of impairment of health related quality of life and symptom severity in IBS has been suggested, but no such data is available from India.

Methods: In this prospective study at a tertiary care center, diagnosis along with sub-classification of IBS was made on the basis of Rome-III criteria. Severity of IBS was graded into mild (score 75-175), moderate (score 175-300) and severe (score 300 to 500) using Irritable Bowel Severity Scoring System (IBSSS). Quality of life was assessed using WHO QOL- BREF which assesses the quality of life in four domains- physical health, psychological, social relationships and environment.

Results: Of 102 IBS patients (32 IBS-C, 40 IBS-D and 30 IBSM); 18 had mild, 57 moderate and 27 had severe symptoms according to IBSSS. Of four domains, physical and psychological domains were most affected. The scores in the physical domain $(58.5 \pm 17.7$ vs. $28.6 \pm 20.8 ; \mathrm{p}<0.01)$ and psychological domain $(62.6 \pm 16.4$ vs. $37.4 \pm 23.3 ; p<0.001)$ were significantly lower in patients with severe disease compared with mild disease severity group. The association of other two domains of quality of life with severity of disease was statistically not significant. There was no statistically significant difference in the quality of life with subtype of IBS or sex of the patient. Conclusion: Physical and psychological domains of quality of life have a direct association with severity of IBS. This further stresses the need for considering these aspects while treating them.

\section{GIM4}

Prevalence of irritable bowel syndrome: A community based study from North India

Govind K Makharia, Anil K Verma, Ritvik Amarchand, Prashant Singh, Abhishek Agnihotri, Anil Goswami, K Anand.

Department of Gastroenterology and Human Nutrition and Centre for Community Medicine, All India Institute of Medical Sciences, New Delhi 110 029, India

Introduction: The prevalence of irritable bowel syndrome (IBS) among Asian communities is rising. The prevalence of IBS varies from $4 \%$ to $20 \%$ in different Asian nations. Prevalence of IBS in native North Indian community is not known.

Methods: We estimated the prevalence of IBS in a rural community in Ballabgarh block in the North Indian state of Haryana from November 2008 to December 2009. A structured questionnaire based on Rome III module was used to collect symptoms related to IBS from all participants in a door to door survey. The diagnosis of IBS as well as further classification based on predominance of symptoms as constipation predominant (IBS-C), diarrhea predominant (IBS-D), mixed symptoms (IBS-M) and unspecified (IBS-U) was done using Rome-III criteria. The estimated sample size for estimation of prevalence was 2515 .

Results: There were 4767 participants (mean age 34.6+ 10.8, males 50\%). Overall, 555 [11.6\% (95\% CI $10.7-$ 12.5)] had constipation, 542 [11.4\% (95\% CI 10.5-12.3)] had diarrhea and 823 [17.3\% (95\% CI 16.2-18.4)] had abdomen pain. 191 fulfilled the criteria for a diagnosis of IBS. Therefore, the overall prevalence of IBS was $4 \%(95 \%$ CI 3.5-4.6). The prevalence of IBS-C was $0.3 \%(95 \% \mathrm{CI}$ $0.16-0.49$ ), IBS-D $1.5 \%$ (95\% CI $1.18-1.90)$, IBS-M $1.7 \%$ (95\% CI 1.35-2.11) and IBS-U 0.5\% (95\% CI 0.32-0.75). Conclusions: The prevalence of IBS in a North Indian community is $4 \%$. 


\section{GIM5}

\section{Role of anorectal manometry prior to colostomy closure}

Akash Shukla, Megha Meshram, Shobna Bhatia. Department of Gastroenterology, Seth G S Medical College and K E M Hospital, Mumbai 400 012, India

Background: Anorectal manometry (ARM) is often done to assess anorectal function prior to colostomy closure. There is a paucity of Indian data about its usefulness. We assessed continence in patients who had ARM prior to colostomy closure. Methods: Sixty four consecutive patients (34 men, age 47.0 [16.6] years) who had undergone ARM (TRACE 1.2, Australia) prior to colostomy closure since January 2009 were included. We considered baseline sphincter pressure $>40 \mathrm{mmHg}$, squeeze pressure $>100 \mathrm{mmHg}$ and squeeze duration of at least $25 \mathrm{~s}$ as adequate, and suitable for colostomy closure. All patients were contacted on phone and their present status recorded.

Results: Thirty one patients had adequate pressures at baseline and were advised colostomy closure; 15 of 19 who underwent colostomy closure were continent, and 4 had urgency, but no incontinence. Thirty three patients had low baseline pressures, and were advised physiotherapy, and 30 did exercises regularly. Repeat manometry showed improvement 7 of 9 patients in whom it was done. Seven of 33 patients with inadequate pressures at baseline underwent colostomy closure; 6 of them were asymptomatic, and one had incontinence. Three of these had pouchitis.

Conclusion: Baseline sphincter pressure $>40 \mathrm{mmHg}$, squeeze pressure $>100 \mathrm{mmHg}$ and squeeze duration of at least $25 \mathrm{~s}$ on ARM appear to be adequate and suitable to prevent incontinence after colostomy closure. Whether lower values will be sufficient needs to be studied.

\section{Liver - Portal hypertension}

\section{LP1}

Experience with endoscopic cyanoacrylate injection of gastric varices to control acute bleed and as secondary prophylaxis at a tertiary care centre

K Kalyan, S Rajanikanth, Shanthivijayaraghavan, Shanmuganathan, Bavanarayana, Somasekar Rao, Rupesh, Seetharam, Naveen.

Sri Rama Chandra Medical College, Porur, Chennai 600 116, India

Bleeding from gastric varices $(\mathrm{GV})$ is more severe and has a higher mortality rate than esophageal variceal bleeding.
Patients with portal hypertension who presented with upper gastrointestinal bleed were assessed after initial stabilization and those who had GOV2 or IGV1 received cyanoacrylate injection in addition to pharmacological therapy. Endoscopic injection was done with a therapeutic endoscope, 21 guage sclerotherapy needle and N-Butyl 2-Cyanoacrylate without any dilution. The maximum amount of glue injected per site was $1 \mathrm{ml}$.Glue was injected at multiple sites until there was solidification of GV. A total of 10 patients presented to our hospital between August 2008 and June 2010 with acute GV bleed. Nine patients had GOV2 and 1 patient had IGV1.Upper GI endoscopy confirmed bleeding from GV. Initial control of bleed was successful in all patients, $(100 \%) .8(80 \%)$ patients had no re bleeding, $2(20 \%)$ had re bleeding. One patient died due to massive ex sanguination. No patient had complications like bacteremia, cerebral or pulmonary emboli. 3 patients are on regular follow up with no further episodes of bleed. Eradication of varices was documented endoscopically for 3 patients. Number of sessions required for variceal eradication were 2 for 2 patients and 3 for 1 patient. In conclusion, the results of our study demonstrate that endoscopic injection of cyanoacrylate is effective not only for controlling acute GV bleed but also for preventing further episodes of bleed.

\section{LP2}

\section{Spontaneous bacterial peritonitis in patients with chronic liver disease with ascites}

Manisha Dwivedi, ${ }^{1}$ SP Misra, ${ }^{1}$ Vatsala Misra, ${ }^{2}$ Sushil Narang, ${ }^{1}$ Sanjay Pant, ${ }^{1}$ D P Rai. ${ }^{3}$

Departments of ${ }^{1}$ Gastroenterology, ${ }^{2}$ Pathology and ${ }^{3}$ Medicine, M L N Medical College, Allahabad 221 001, UP, India

Aims: To determine the prevalence, microbial spectrum and outcome in patients with spontaneous bacterial peritonitis (SBP) and its variants.

Methods: A total of 120 patients with CLD with ascites were included in this study. Patients were subjected to abdominal paracentesis for routine examination and ascitic fluid culture by direct inoculation of blood culture bottles at the bedside. The patients were divided into SBP, culture negative neutrocytic ascites (CNNA) and bacterascites groups in the light of results of ascitic fluid examination. Results: The study included 89 males and 31 females having CLD with ascites. The mean age was $44.40 \pm 16.32$ years. Thirty one of $120(26 \%)$ patients had SBP and its variants. SBP was diagnosed in $13(11 \%)$ patients where as CNNA and monomicrobial bacterascites were detected in $17(14 \%)$ cases and one $(0.83 \%)$ case respectively. E.coli was the most frequently cultured organism, isolated in $12(85 \%)$ cases with SBP. One case of Klebsiella species (7\%) and one of Staphylococcus aureus (7\%) were reported. Eleven (85\%) patients with SBP belonged to 
Child-Pugh Class C and 2 (15\%) belonged to Class B. In the SBP group, five patients $(n=13,38 \%)$ expired and $8(n=13,62 \%)$ improved during hospital stay where as in the non SBP group11 $(n=107,10 \%)$ patients expired during hospital stay and 96 ( $n=107,90 \%$ ) patients improved and were discharged.

Conclusion: SBP is a common complication of CLD with ascites and is associated with significant in-hospital mortality. E. coli is the most frequent cultured organism.

\section{LP3}

\section{Ischemic complications of Terlipressin in liver disease:} a case series

\section{Manish Jain, Sanjay Kumar, Sandesh Sharma, Arvind Namdev, Neeti Jain, Neetu Kumar. \\ Global Liver and Gastroenterology Centre, MP, India}

Terlipressin is a synthetic analogue of vasopressin, which has been used in the treatment of acute variceal hemorrhage. In contrast to vasopressin, terlipressin can be administered as intermittent injections instead of continuous intravenous infusion. Thus, it has a less adverse reaction than vasopressin. Aim: To report two cases of skin necrosis in cirrhotic patients treated with the vasoconstrictor agent terlipressin (Glypressin). Methods: We identified two patients who developed skin necrosis and determined any factors, which put them at an increased risk of doing so.

Results: One patient was obese and developed abdominal wall skin necrosis. The second patient had lower limb chronic venous insufficiency and developed necrosis of both lower limb skin.

Conclusions: With increasing clinical use of terlipressin and an increasing incidence of obesity and non-alcoholic fatty liver disease-related cirrhosis, the incidence of these serious complications is likely to rise. Earlier recognition and treatment may lead to improved outcome.

\section{LP4}

\section{Role of propranolol in non-cirrhotic portal hypertension}

B Sivasubramaniam, VArul Selvan, Arun Kumar, V Jayanthi. Department of Gastroenterology, Stanley Medical College Hospital, Chennai 600 003, India

Background: Propranolol reduces portal hypertension by causing splanchnic vasoconstriction. It is recommended for secondary prophylaxis in bleeders with grade III and IV oesophageal varices. The role of propranolol in extrahepatic portal vein obstruction (EHPVO), and non cirrhotic portal fibrosis (NCPF) in preventing esophageal variceal bleed is not clear. Aim of the study: To determine the role of propranolol in variceal bleed in patients with EHPVO and NCPF.
Methods: Patients with EHPVO (Gp I), NCPF (Gp II) and Cirrhosis (Gp III) were included for the study. The estimated sample size is 50 in each group. Age, gender, details of first and subsequent bleed, grades of varices and details of intervention were retrieved from the records. Patients on propranolol were included for the study. Those with fundal varices and Child $\mathrm{C}$ cirrhosis were excluded. Results: The mean age among the 3 groups was $27 \mathrm{yrs}, 43 \mathrm{yrs}$ and 50 yrs respectively. Female predominance was observed in Gp II. The three groups were matched for demographic data and grades of varices. Following endotherapy for varices and secondary prophylaxis with propranolol, $60 \%$ in Gp I, $47.8 \%$ in Gp II and (31.7\%) in Gp III had subsequent bleed during mean follow up period of 5.52, 2.325 and 2.375 yrs respectively. Conclusion: Subsequent bleed was common in EHPVO despite adequate intervention and secondary prophylaxis with propranolol, compared to patients with NCPF and liver cirrhosis. Propranolol does not appear to reduce variceal bleed risk in EHPVO. Periodic screening and intervention for recurrence of esophageal varices in EHPVO seems mandatory.

\section{LP5}

A Case report-ileocolic varices - a result of AV fistulae

Ajith Roni, Sathish Kumar, Pavan Kumar Reddy, Lalit Nihal. Department of Medical Gastroenterology and Hepatology, Narayana Medical College, Nellore, India

A 65-year-old male presented with malena for 3 days with out postural symptoms. He had no history of liver disease or NSAID intake. He had hematochezia 2 years ago. He had no comorbidities or surgery in the past. Clinical examination revealed mild pallor with normal general and systemic examination. His hemoglobin was $10 \mathrm{gm} \%$. Gastroscopy was normal. Colonoscopy showed varices from rectum to terminal ileum sparing transverse colon. Routine blood investigations were normal. Ultrasound abdomen and Doppler of abdominal veins were normal. CECT abdomen showed ectatic, tortuous, abnormally enhancing tuft of vessels in jejunum, ascending colon and terminal ileum suggestive of arteriovenous (AV) fistulae. Vascular lesions of gastrointestinal (GI) tract are divided into $\mathrm{AV}$ fistulae and AV malformations. AV fistulae are arteriovenous and arterioportal. They may be a major source of GI bleeding. Arterioportal fistulae are more common than AV fistulae. They cause extrahepatic portal hypertension (PHTN) causing varices. Diagnosis is by CECT abdomen or angiography. The definitive treatment is radiological embolisation of fistulae or surgery. We report this case as it is rare and it is important to distinguish the varices resulting from PHTN of liver disease. This is the first case report of this kind from India. There are several case reports of Isolated colonic and ileocolic varices but most of them are in cirrhotics or due to traumatic AV fistulae. 


\section{LP6}

Serum tumor necrosis factor alpha level in HEV related acute viral hepatitis and fulminant

Salam Gyaneshwori Devi, ${ }^{1}$ Ashok Kumar, ${ }^{1}$ Premashis Kar. ${ }^{2}$ Department of ${ }^{1}$ Obstetric \& Gynaecology and ${ }^{2}$ Department of Medicine, Maulana Azad Medical College \& Lok Nayak Hospital, New Delhi 110 002, India

The host response in HEV related liver disease of pregnant women is unclear. This prospective study was carried out to evaluate the role of tumor necrosis factor alpha (TNF-a) in HEV related acute viral hepatitis (AVH) and fulminant hepatic failure (FHF) in pregnant women. The study included HEV related pregnancy cases: $24 \mathrm{AVH}$ and $10 \mathrm{FHF}$; Non-HEV related pregnancy cases: $48 \mathrm{AVH}$ and $3 \mathrm{FHF}$; HEV related non pregnant cases: $16 \mathrm{AVH}$ and $4 \mathrm{FHF}$; Non-HEV related non pregnant cases: $44 \mathrm{AVH}$ and $5 \mathrm{FHF}$; 60 healthy pregnant women and 50 healthy non pregnant women. Serum TNF-a level was assayed by commercially available ELISA kits according to manufacture's protocol. Pregnant cases had significantly higher HEV viral load in both AVH and FHF cases than non pregnant cases $(p=0.0001$ and 0.044 respectively). Increase level of TNF-a was observed in $\mathrm{AVH}$ and FHF of HEV infected pregnant women than the Non-HEV pregnant women $(p=0.001$ and 0.106 respectively). TNF-a level was significantly higher in AVH and FHF of HEV infected pregnant women compared with AVH and FHF of HEV infected non pregnant women $(p=0.006$ and 0.001 respectively) and healthy pregnant women $(p=0.0004$ and 0.0001 respectively). Increase TNF-a level was observed in HEV infected women having preterm delivery and low birth weight newborn compared with Non-HEV and healthy pregnant women. In conclusion increase serum concentration of TNF-a observed in HEV infected FHF and AVH pregnant cases shows that pregnancy with HEV infection increases TNF-a secretion. TNF-a might be an important factor in the different outcomes of HEV infection during pregnancy.

\section{LP7}

High HVPG and low platelet predict post endoscopic variceal ligation ulcer bleed in patients with cirrhosis

Vibhu Vibhas Mittal, Ashish Kumar, Nitin Gupta, Ram Soni, Vishal Garg, Barjesh Chander Sharma, Shiv Kumar Sarin.

Department of Gastroenterology, G B Pant Hospital and Department of Hepatology, Institute of Liver and Biliary Sciences, New Delhi, India

Background: Post endoscopic variceal ligation (EVL) ulcer bleed is a significant problem with limited treatment options and high mortality. Factors leading to development of EVL ulcers have been studied but those predicting bleed have not been investigated.

Aim: To study the predictive factors of post EVL ulcer bleed Methods: Consecutive cirrhotics undergoing EVL were included in the study. Baseline demographic, clinical, endoscopic and laboratory data were collected. Baseline hepatic vein pressure gradient (HVPG), whenever possible was measured. Patients were followed up with weekly endoscopy till bleed or resolution of ulcer.

Results: 60 cirrhotics underwent EVL during the study period, for a total of 114 procedures. Mean age was $44.24 \pm 12.5 \mathrm{yr}$; $78.2 \%$ were males. Etiology of liver disease was alcohol $(36.7 \%)$, viral $(28.3 \%)$ or others $(35.0 \%)$. EVL was done in the setting of acute bleed in $30.0 \%$ and for prophylaxis of variceal bleed in $70.0 \%$. Four patients had HCC. HVPG was measured in 32 patients (mean $21.34 \pm 4.4 \mathrm{~mm} \mathrm{Hg}$ ). Esophageal ulcers developed in all patients. Eight (7.01\%) patients had an ulcer related UGI bleed, within a median duration of 6 days. On univariate analysis, large size of esophageal varices, low platelet count (p-.031), high pulse rate before EVL (p-.045), high total leukocyte count (p-.035), high HVPG (p-.035) and HCC status (p-.035) were significantly associated with occurrence of bleed. In multivariate analysis after excluding HVPG, presence of HCC and low platelet count were associated with occurrence of bleed.

Conclusion: Post EVL ulcers are universal, but they are associated with bleeding in patients with HCC, high HVPG and low platelet count.

\section{LP8}

Propofol sedation during endoscopy in patients with cirrhosis and utility of psychometric and critical flicker frequency in assessment of recovery from sedation

Praveen Sharma, Shweta Singh, ${ }^{*}$ Manoj Kumar, Hitender Garg, Ashish Kumar, Barjesh Chander Sharma, Shiv Kumar Sarin.

Department of Hepatology and Anesthesia, *Institute of Liver and Biliary Sciences Vasant Kunj, Delhi, India

Introduction: Patients with cirrhosis could be at increased risk of complications. We assessed the utility of critical flicker frequency (CFF) in recovery of patient with cirrhosis after endoscopy with propofol.

Methods: Consecutive patients with cirrhosis who underwent endoscopy under propofol were enrolled. Patients underwent number connection test-A and B (NCT-A, B); digit symbol test (DST), line tracing test (LTT) and serial dotting test (SDT) before and $2 \mathrm{hrs}$ after endoscopy. CFF was done at baseline before endoscopy and then at every $30 \mathrm{~min}$ for the next $2 \mathrm{hrs}$. 
Results: Of 108 patients there was no deterioration in psychometry tests before and after endoscopy [NCT-A $(65.2 \pm 44.4$ vs. $62.4 \pm 43.6 \mathrm{sec}, p=0.01)$, NCT-B $(110.4 \pm$ 34.7 vs. $109.6 \pm 44.6 \mathrm{sec}, p=0.45)$, DST $(26.2 \pm 10.0$ vs. $26.7 \pm 9.9, p=0.25)$, LTT $(116.6 \pm 55.2$ vs. $115.4 \pm 51.3 \mathrm{sec}$, $p=0.47)$, SDT $(88.6 \pm 47.5$ vs. $84.3 \pm 44.1 \mathrm{sec}, p=0.02)]$. Patients with minimal hepatic encephalopathy (MHE, $n=$ 64) did not show any deterioration in psychometric tests at $2 \mathrm{hr}$ [NCT-A $(87.7 \pm 45.4$ vs. $84.3 \pm 44.9 \mathrm{sec}, p=0.06)$ NCTB (134.8 \pm 65.4 vs. $132.7 \pm 58.8 \mathrm{sec}, p=0.46)$, SDT $(107.1 \pm$ 53.0 vs. $102.7 \pm 48.5 \mathrm{sec}, p=0.03)$, DST (21.4 \pm 8.9 vs. $22.2 \pm 8.8, p=0.09)$ and LTT $(131.5 \pm 62.2$ vs. $129.6 \pm$ $57.2 \mathrm{sec}, p=0.46)]$. There was significant difference between CFF at baseline and at $30 \mathrm{~min}$ and $1 \mathrm{~h}$ but no difference thereafter in whole group, in MHE patients and in controls. Thirty (28\%) patients had CFF $<38 \mathrm{~Hz}$ and CFF at $2 \mathrm{hr}$ did not significantly differ from baseline CFF $(35.9 \pm 1.5$ vs. $36.1 \pm 2.0 \mathrm{~Hz} p=0.19)$ though it was significantly low at $30 \mathrm{~min}(34.6 \pm 2.5, p=0.001)$ and at $1 \mathrm{hr}(35.0 \pm 1.7, p=0.005) .10(9 \%)$ patient had transient hypoxemia and $18(17 \%)$ had hypotension during procedure but endoscopy completed in all.

Conclusions: Propofol is safe in cirrhotics patients and $\mathrm{CFF}$ is a useful tool to assess recovery from sedation.

\section{LP9}

A study to assess efficacy and safety of $n$ "butyl -2- in patients with bleeding gastric varices

P Anita, K Panduranga Rao, K Venugopal Reddy, $G$ Mohan Reddy, K Sathyanarayana, S T Rajanesh Reddy. Department of Gastroenterology, Osmania Medical College, Hyderabad, AP, India

Objectives: To assess efficacy and safety of cyanoacrylate injection in patients with bleeding gastric varices.

Methods: 20 patients [16-male, 4-female; age group 3550 years] with gastric variceal bleed, who underwent endoscopic cyanoacrylate injection therapy, over a period of one year were included in this study. Hemostasis, Recurrent bleeding rate, related complications were studied. Patients were followed every quarterly for a period of 1 year.

Results: Out of the 20 patients studied, Hemostasis was achieved with initial endoscopic therapy in 17 patients. Initial success rate was $85 \%$. Recurrent bleed developed in 3 of these 17 patients, which was managed with repeat injection. Overall rate of rebleed was 20\%. 3 patients died [uncontrolled bleed]. Overall mortality rate was $15 \%$. Minor complications occurred in 8 of treated patients [5 - retrosternal pain, 3 - ulcers at injection site].
Conclusions: 1. Cyanoacrylate is efficacious for treating bleeding gastric varices. 2 . Recurrent bleed can successfully be treated with repeat cyanoacrylate injection. 3. Cyanoacrylate injection is relatively safe with only minor complications.

\section{LP10}

Addition of probiotics to propranolol improves response for primary prophylaxis of variceal bleeding in patients with cirrhosis and large esophageal varices

Nitin Gupta, Shiv K Sarin, Barjesh C Sharma, Vishal Garg, Ashish Kumar.

Department of Gastroenterology, G B Pant Hospital, New Delhi

Background and Aims: Probiotics favourably alter gut flora and decrease endotoxemia in cirrhosis. This double blind RCT evaluated the effects of probiotic administration on the hepatic venous pressure gradient (HVPG) and its safety in patients with cirrhosis and portal hypertension.

Methods: 87 patients with cirrhosis and portal hypertension (HVPG $\geq 12 \mathrm{mmHg}$ ) with large esophageal varices $(90 \%$ high risk, red color signs), who were non bleeders, were randomized to receive either: Propranolol + Placebo (Group I, $n=30$ ), Propranolol + Norfloxacin (400 mg BD; Gr. II, $n=27$ ) or Propranolol + vsl\#3 (900 bspores/day; Gr. III, $n=$ 30). Propranolol was titrated to achieve heart rate of $55 \mathrm{bpm}$ or maximum of $320 \mathrm{mg} /$ day. We studied splanchnic hemodynamics before and after 2 months of treatment. Primary end point was change in HVPG at 2 months compared with baseline. Secondary end point was the number of adverse events.

Results: Mean age was 43 y, 76\% males. Median MELD score was 8 and CTP score was 8 . Median grade of esophageal varices was 3 . The 3 groups were comparable to each other. Addition of vsl\#3 to propranolol improved primary end-point, the response rate ( $\geq 20 \%$ fall in HVPG) more compared to group $1(58 \%$ vs. $31 \%, p=0.046)$; while it was slightly increased in group 2 compared to group 1 (50\% vs. $31 \%, p=0.08)$. The fall in HVPG levels was higher in group 3 compared to group 1 (3.7 vs. $2.1 \mathrm{~mm} \mathrm{Hg}$, $p=0.06$ ). Norfloxacin decreased HVPG more compared to propranolol alone but not to the extent as by vsl\#3 (3.3 mmHg vs. $2.1 \mathrm{mmHg}, p=0.13$ ). Number of patients with adverse events were similar in the 3 groups. Two patients in group 3 were withdrawn because of side effects of propranolol. One patient in each group had UGI bleed.

Conclusions: Addition of Vsl\#3 to propranolol synergistically increased the degree of reduction of HVPG in patients with cirrhosis with large varices. Vsl\#3 was well tolerated. Its beneficial effects should be confirmed in further clinical trials with larger sample size and a higher dose. 


\section{Liver - Viral Hepatitis}

\section{LV1}

\section{An audit of hepatitis $C$ cases seen over 8 years in a referral Railway hospital in Eastern India}

\section{Gautam Ray, Santanu Mondal.}

Gastroenterology Unit, Department of Medicine, B R Singh Hospital, Eastern Railway, Kolkata

Methods: 84 consecutive AntiHCV+ [OPD and indoor] cases diagnosed from June 2002 to June 2010 were evaluated for clinical presentation and treatment outcome. Results: Mean age [S.D, Range] was 53.48 [12.56, 8-78] years, male age $53.07[15.45,18-68]$ years, female age 53.69, [11.03, 8-78] years, M:F:: 55:27. Median [range] HCV RNA level in 46 cases done was 80,000 [500-107] copies $/ \mathrm{ml}$ and genotype $1=7[15 \%], 2=1[2 \%], 3=36[79 \%]$, $4=1[2 \%], 6=1[2 \%] .35[42 \%]$ presented as decompensated disease, 6[7\%] had HCC. Concomittant HBV infection was present in $3[3.5 \%]$, diabetes mellitus in $6[7 \%]$, alcohol use in 4[4.5\%]. All spouses were AntiHCV negative. Meaningful follow up was possible in 44 [51\%] cases, mean [S.D, range] $3.3[1.2,1 / 2-8]$ years. 5 [6\%] died. 37 qualified for treatment with curative intent, 34 were given Peginterferon + ribavirin, 29 had completed therapy of which $24[83 \%]$ had SVR. 3 received interferon + ribavirin, 2[67\%] had SVR.

Conclusion: Majority of treatable $\mathrm{HCV}$ cases are of genotype 3 in east India with good treatment outcome but a significant proportion present with decompensated disease precluding curative drug therapy.

\section{LV2}

\section{Intensive nutritional intervention improves hypoproteinemia in decompensated liver cirrhosis}

PreetiSrivastava, Prerna Singh, Divya Singh, Gourdas Choudhuri. Department of Gastroenterology, Sanjay Gandhi Postgraduate Institute of Medical Sciences, Lucknow 226 014, India

Background and Aim: Hypoalbuminemia is frequent and often severe in patients with Liver Cirrhosis (LC). We evaluated whether intensive nutritional intervention could improve albumin synthesis and blood levels in patients with advanced LC.

Method: Four hundred and ninety-five patients (male 381, mean age $45 \pm 10.9$, Child A: 142, Child B: 133, Child C: 109) of LC recruited for the study were stratified according to Child-Pugh class and randomized to receive one of 3 nutritional inputs: (a) usual nutritional counselling with the help of a leaflet, (b) intensive nutritional counselling by a trained nutritionist and dietary intervention with homemade feeds (c) Intensive nutritional counseling as above in with additional supplementation with protein formula feeds to account for atleast $25 \%$ of total daily calories. Prealbumin and albumin levels were measured at baseline and at 3 months. Paired $t$ test was used for analysis.

Result: Type c diet showed maximum increase, over baseline, in prealbumin (Child A:10\%, Child B:17\%, Child C:16.6\%) and albumin levels (A: 3\%, B:7\%, C:4\%). The corresponding values for $\mathrm{b}$ diet for pre-albumin (A:9.5\%, $\mathrm{B}: 13 \%, \mathrm{C}: 8 \%$ ) and albumin (A: $0 \%, \mathrm{~B}: 3.2 \%, \mathrm{C}: 0 \%$ ) were less. The a diet fared worst with minimal increase in prealbumin (A:5\%,B:4\%,C:3.4\%) and albumin (A:0\%, B:3.3\%, C:0\%) levels.

Conclusion: Our study showed that intensive nutritional intervention by a trained nutritionist and feeding of high protein diet supplemented with formula-feeds produced the maximum increase in serum levels of pre-albumin and albumin. The benefit was most evident in patients with decompensated (Child B \&C) liver cirrhosis.

\section{LV3}

\section{Role of glutathione S-transferase M1 and T1 gene polymorphism, hepatitis $B$ and $C$ virus infection and aflatoxin B1-related hepatocellular carcinoma in India}

Mohammad Asim, ${ }^{1}$ Manash P Sarma,${ }^{1}$ L Thayumanavan, ${ }^{1}$ P Kar. ${ }^{l}$

${ }^{1}$ Department of Medicine, Maulana Azad Medical College, University of Delhi, New Delhi 110 002, Department of Gastroenterology, Madurai Medical College, Madurai 600 020, Tamil Nadu, India

Objective: Our aim was to evaluate whether the association of GSTM1/T1 gene polymorphisms modifies the risk of Hepatocellular carcinoma (HCC) and its correlation with Aflatoxin B1 and hepatitis B and C infections.

Methods: It was a case-control study, included $254 \mathrm{HCC}$ cases compared with 265 hospital-based age and sex matched cases of chronic liver disease without HCC as controls from Indian population. The GSTM1 and GSTT1 genotypes were detected using conventional multiplex PCR method. The level of aflatoxin B1 in the food samples collected at the recruitment of patients was examined by competitive enzyme-linked immunosorbant assay.

Results: We observed a positive correlation between age, Aflatoxin $\mathrm{B} 1, \mathrm{HBV} / \mathrm{HCV}$ infection, smoking habit $(>20$ packs/year), alcohol consumption ( $>100 \mathrm{~g} /$ day) and risk of liver cancer. We found significantly increased risk associ- 
ated with GSTM1 null genotype $(\mathrm{OR}=3.49)$ as well as GSTT1 null genotype $(\mathrm{OR}=3.12)$, respectively. The biological gradients between Aflatoxin B1 level and HCC risk were observed among HBV patients who had null genotypes of GST Ml and/or T1 but not among those who had non-null genotypes. However, an increased risk of HCC was observed among heavy drinkers with GSTM1 $(\mathrm{OR}=2.01)$. Further, cigarette smoking showed a nonsignificant association with GSTT1 $(\mathrm{OR}=1.49)$.

Conclusion: Our results suggest that the variants in low penetrance gene such as GSTM1 and GSTT1 are associated with an increased liver cancer risk. Further, an influence of GSTM1/T1 null genotypes may contribute in the etiology of HCC in patients with higher levels of Aflatoxin B1, extensive cigarette and alcohol consumption, respectively.

\section{LV4}

To determine the clinical and virological profile of incidentally detected asymptomatic hepatitis B positive individuals

Meghraj Ingle, Abhijit Deshmukh, Hardik Shah, Devendra Desai, Philip Abraham, Anand Joshi.

P D Hinduja National Hospital and Medical Research Centre, Mahim, Mumbai 400 016, India

Introduction: India has around 45 million people affected with hepatitis B. Incidentally detected asymptomatic hepatitis B positive subjects (IDAHS) forms a large group within these patients. The guidelines on management of IDAHS are changing.

Aims: To prospectively study the clinical and virological profile of IDAHS at our centre over one year.

Methods: All IDAHS were evaluated prospectively by history, clinical examination and investigations (HBV viral load, $\mathrm{HBeAg}$, Anti-HBe, alanine transaminase (ALT), USG and liver biopsy (abnormal ALT or high HBV DNA Load). Patients were followed up for one year with ALT every 3 months.

Results: Of 85 IDAHS (65 males; mean age 40.2 years), 6 (7\%) were HBeAg and 78 (91.76\%) Anti-HBe positive. Twenty-two (26\%) had abnormal baseline ALT and twentyone $(24.7 \%)$ developed abnormal ALT during one year follow up. High baseline HBV DNA ( $>20000 \mathrm{IU} / \mathrm{ml})$ was found in $14(16.4 \%)$. Abnormal ALT was more frequent than high HBV DNA $(p=0.003)$ Biopsy was indicated in $21(25.6 \%)$ patients of which 11 consented and $10(11.7 \%)$ had HAI $>3$. Abnormal histology positively correlated with high baseline HBV DNA $(p=0.0001)$ but not with HBeAg positivity $(p=0.6)$, abnormal ALT $(p=0.7)$, age $>40(p=$ $0.22)$. Twelve $(14.1 \%)$ were started on treatment (2 without biopsy).
Conclusions: One half of IDAHS had abnormal ALT or developed during follow up. High HBV viral load was found in $16 \%$ patients. Liver biopsy was indicated in about one forth of patients. Ninety percent of patients undergoing biopsy had significant inflammation needing treatment. Abnormal histology correlated with HBV viral load only.

\section{LV5}

Study of DNA repair genes polymorphism and its association with liver disease susceptibility in Northeast India

Moumita Bose. ${ }^{1}$ Bharati Baruah, ${ }^{1}$ Anjan Saikia, ${ }^{2}$ Rajkumari Deblakshmi, ${ }^{1}$ Purabi Deka Bose, ${ }^{1}$ Subhash Medhi, ${ }^{1}$ Sujoy Bose, ${ }^{1}$ Manab Deka. ${ }^{1}$

${ }^{1}$ Deparment of Biotechnology, Gauhati University, Guwahati, Assam, ${ }^{2}$ Central Hospital, N F Railway, Maligaon, Guwahati, Assam

Genetic alteration in BER pathway genes, and oxidative stress induced by hepatitis infection may predispose patients toward liver disease susceptibility; with no data from ethnically distinct Northeast Indian (NEI) population. Aim: Study association of genetic variation of BER genes hOGG1ser326cys, XRCC1codon399; and oxidative stress by virtue of 8-OH-dG status in liver disease susceptibility. Method: Liver disease patients $(n=170)$ of viral, alcoholic and cryptogenic aetiology from Central Railway Hospital, Guwahati; and 170 age-sex matched community controls were enrolled for the study. hOGG1ser326cys and XRCC1codon399 gene polymorphism was studied by PCR-RFLP method. Oxidative stress was measured in plasma using 8-OH-dG ELISA kit.

Results: Higher percentage of mutant allele was present in patients compared to controls for hOGG1ser326cys $(75.29: 57.06, p<0.001)$ and XRCC1codon 399 (65.29:55.29, $p=0.06)$ gene. The risk of liver disease was increased by the presence of mutant alleles of hOGG1 $\{\mathrm{OR}=2.294(1.445-3.641)\}$ and XRCC1 $\{\mathrm{OR}=1.521$ (0.982-2.355)\}. Higher concentration of $8-\mathrm{OH}-\mathrm{dG}$ was found in plasma of both patients $(474.34 \pm 207.54 \mathrm{pg} / \mathrm{ml})$ and controls $(327.63 \pm 194.87 \mathrm{pg} / \mathrm{ml})$ compared to the recommended levels of 4-21 pg/ml. 8-OH-dG concentration was highest in HAV cases and was significant for alcoholic patients $(p<0.03)$.

Conclusion: Higher prevalence of mutated genotype of BER pathway genes may play a crucial role in liver disease susceptibility. Also, presence of high percentage of $h O G G 1$ mutation and higher 8-OxoG are direct indication of DNA damage and oxidative stress related liver damage in NEI population. 
LV6

Acute hepatitis C; case series; hit early and hard?

Agarwala Mukesh, Dhus Ubal, Rao Subba.

Department of Gastroenterology, Apollo Hospitals, Chennai, India

Background: Acute hepatitis $\mathrm{C}$ is rare. No standard treatment regimen defined for acute $\mathrm{HCV}$ infection. Early treatment with Interferon monotherapy prevent chronicity. Here we present six patients with acute HCV infection.

Methods: We included six patients with acute $\mathrm{HCV}$ infection. All patient had CKD on regular hemodialysis, who were detected to have recent $\mathrm{HCV}$ infection (either Anti HCV positive or HCV NAT positive), we did HCV RNA quantitative by RT PCR in each case. Patients with HCV RNA $>100000$ copies/ml were treated with interferon (Three patients with PEG interferon and one with standard interferon) and all were followed up with repeat HCV RNA after an interval of 4-6 weeks.

Result: Out of six patients, male female ratio was 4:1, age ranging from $30-50$ years. Five patients had HCV RNA viral load $>100000$ copies $/ \mathrm{ml}$ and one patient had HCV RNA.

\section{LV7}

The occurrence and pattern of HBsAg positivity in family members of $\mathrm{HbsAg}$ positive patients and burden of liver disease in these family members

R Kavitha, KSunilKumar, TMRamachandran, Varghese Thomas. Department of Gastroenterology and Internal Medicine, Medical College Calicut, Kerala

Aim: To evaluate the occurence of HBV infection in family members of HBV positive patients, and evaluate the burden of liver disease in the family members.

Methods: All HBsAg positive patients attending ourdepartment, for last $1 \frac{1}{2}$ yrs were included in study. The index case was evaluated withHBeAg, anti $\mathrm{HBe} \mathrm{Ab}, \mathrm{HBV}$ DNA, LFT and USG abdomen. All first degree relatives were screened and those who tested positive were evaluated for disease activity.

Results: Total of 268 index cases were imcluded in study. $\mathrm{M}: \mathrm{F}=1.4: 1$.Mean age was $32.17 \mathrm{yrs}(6-75 \mathrm{yrs}) .86$ of 268 patients $(32.09 \%)$ didn't turn up after initial visit. Among the remaining 182 patients, 36 (19.78\%) were $\mathrm{HBeAg}$ positive. LFT abnormalities were seen in 98 $(53.85 \%)$. Out of $182,38(20.8 \%)$ had established cirrhosis. 5.9\% had acute hepatitis. Among the family members screened, $41.7 \%$ had positive family members (76/182). Among the 76 cases with family members affected, 19 (25\%) was HBeAg positive. Brothers (50\%) and sisters $(35.5 \%)$ were most commonly affected. Mothers of the index cases affected were $19.74 \%$ and fathers $27.63 \%$. Among the affected family members, $42.9 \%$ had LFT abnormality and $7 \%$ had underlying cirrhosis.

Conclusion: The occurrence of hepatitis B positivity in family members of HBV positive patients was $41.7 \%$. Brothers and sisters were more affected and this could not be corroborated as only $19.74 \%$ of mothers were positive for HBV infection. Hence the infection among siblings and parents may be due to horizontal transmission.

\section{LV8}

\section{Nitric oxide synthase and hepatitis $E$ infection}

Rajib K Hazam, Purabi D Bose, Subhash Medhi, Manash P Sarma, Premashis Kar.

Department of Medicine, Maulana Azad Medical College, New Delhi 110 002, India

Background and Aim: Nitric oxide (NO) is one of the most versatile mediators in the control of viral infections, being the earliest host's antiviral response as well as in the pathogenesis of many human infections, makes it reasonable to consider this molecule as a potential player in Hepatitis E virus (HEV) infection. Correlation between iNOS, eNOS polymorphism and expression and disease severity in hepatitis B related hepatitis has been reported earlier. The study was aimed to determine the role of iNOS and eNOS gene polymorphism and expression in HEV related liver disease.

Methods: 197 AVH, 37 FHF age and sex matched 256 healthy controls were included. PCR amplification for iNOS and eNOS genes was performed using specific primers. RFLP analysis was carried out by restriction digestion using enzymes TSP509I for iNOS and Ban II for eNOS to detect the polymorphism. Expression study was done using commercially available ELISA kits and by rtPCR.

Results: The frequency of iNOS (CT + TT) and eNOS $(\mathrm{GT}+\mathrm{TT})$ genotypes was non significantly higher in FHF compared to $\mathrm{AVH}$ and healthy controls. iNOS and eNOS expression in FHF[ $56.5 \pm 6.2 \mathrm{IU} / \mathrm{ml}, 61.1 \pm 1 \mathrm{IU} / \mathrm{ml}$ ] was significantly increased as compared to AVH[717.2 \pm $8 \mathrm{IU} / \mathrm{ml}, 23.3 \pm 4.2 \mathrm{IU} / \mathrm{ml}]$ and controls $[7.7 \pm 3.2 \mathrm{IU} / \mathrm{ml}, 16 \pm$ $3.6 \mathrm{IU} / \mathrm{ml}$ both by ELISA $(p<0.005)$ and semi quantitative rt-PCR.

Conclusion: iNOS and eNOS may be a potential determinant of susceptibility to fulminant hepatitis. 


\section{LV9}

\section{Association of anemia with liver disease patients: A new Delhi experience}

Jayanta Borkakoti, Manash Pratim Sarma, Mohammad Asim, Rajib Kishore Hazam, Premashis Kar.

Department of Medicine, Maulana Azad Medical College, New Delhi 110 002, India

Introduction: Liver diseases are frequently associated with haematological abnormalities. The risk of acquiring both hepatitis B virus (HBV) and hepatitis C virus (HCV) infections in patients with hematological-oncological disorders has been documented.

Aim: To assess the prevalence of HBV and HCV infection in anaemic patients by immunological and molecular assays, risk factors associated and clinical status.

Methods: In a retrospective study 40 patients were found with complaints of anaemia and infection with some hepatotropic viruses. The age, sex, LFT profiles of such patients were observed.

Results: 30 out of 40 patients were female $(75 \%)$ with a male: female ratio of $1: 3$ and mean age 25.26 . HbsAg was positive in $5(12.5 \%)$ while anti $\mathrm{HCV}$ was positive in 9 cases $(22.5 \%)$. The mean Hb was 6.02, SGOT-244, SGPT-251. Mild fever was documented in $29(72.5 \%)$, anorexia in $27(67.5 \%)$, Nausea in $23(57.5 \%)$, Vomiting in $22(55 \%)$, abdominal discomfort in $22(55 \%)$, malena in $13(32.5 \%)$, blood transfusion in $10(25 \%)$,ascites in $6(15 \%)$ and pedal edema was in 8 cases $(20 \%)$.No case was documented with drug abuse, professional blood donors, multiple sexual partners or alcohol intake. 3 cases were aplastic anemia, 2 megaloblastic anemia, 1 dimorphic anemia and 9 with severe anemia.

Conclusion: HbsAg was positive in $12.5 \%$ while anti $\mathrm{HCV}$ was positive in $22.5 \%$ cases. Silent HCV infection was documented in many researches which need to be studied. The pathogenesis of the anemia in each case is different and needs correct therapy.

\section{LV10}

HBeAg negative status in classical acute hepatitis B virus infection in southern India-A prospective study

P N Rao, ${ }^{1} P$ Balkumar Reddy, ${ }^{2} R$ M Mukherjee, ${ }^{2}$ M Sasikala, ${ }^{2}$ Rajesh Gupta, ${ }^{1}$ D Nageswar Reddy. ${ }^{1}$

${ }^{1}$ Asian Institute of Gastroenterology, ${ }^{2}$ Asian Health Care Foundation, Somajiguda, Hyderabad 500 082,India

Introduction: Distinction between a true episode of acute hepatitis $\mathrm{B}$ (AHB) and an acute flare of chronic hepatitis $\mathrm{B}$ (CHB-AF) is often difficult in endemic settings of hepatitis B virus (HBV) infection. While HBeAg seroconversion occurs early in AHB but delayed in CHB, detection of anti-HBc IgM is the hallmark of acute HBV infection. Since $\mathrm{HBeAg}$ status is not usually monitored routinely in classical AHB patients, this study is aimed at finding the prevalence of $\mathrm{HBeAg}$ negative state in $\mathrm{AHB}$ patients at registration and subsequent follow-up for six months.

Methods: 34 patients having clinical features of AHB infection were prospectively enrolled and followed up for six months. Serum ALT, HBsAg, HBeAg, anti-HBe and anti-HBc IgM status were evaluated by standard biochemical and ELISA techniques.HBV-DNA quantification was performed by real-time PCR (Roche Diagnostics,USA)assay. Results: All patients were positive for anti HBcIgM. 77\% were $\mathrm{HBeAg}$ negative and simultaneously positive for anti HBe. Serum HBV DNA and ALT levels were elevated, mainly in elder subjects, at 1-2 week and started declining at 4 th week of $\mathrm{HBeAg}$ seroconversion. After six months follow-up studies,majority (59\%)of these patients cleared HbsAg with non detectable level of HBV DNA and normalized ALT values.

Conclusion: HBeAg negative status in classical AHB is not uncommon due to early seroconversion. Presence of Anti HBcIgM and clearence of serum HBV DNA at follow-up in these patients,indicate AHB than CHB-AF.The chance of occurrence of CHB-AF in older patients with delayed $\mathrm{HBeAg}$ seroconversion is discussed.

\section{LV11}

Quantitation of serum HBsAg in chronic hepatitis B virus infection by sandwich ELISA

P Balkumar Reddy, ${ }^{I} R$ M Mukherjee, ${ }^{1}$ Jyothi Arava, ${ }^{1}$ P N Rao, ${ }^{2}$ M Sasikala, ${ }^{1}$ Rajesh Gupta. ${ }^{2}$

${ }^{1}$ Asian Health Care Foundation, ${ }^{2}$ Asian Institute of Gastroenterology, Somajiguda, Hyderabad 500 082, India

Introduction: Qualitative detection of hepatitis B virus surface antigen (HBsAg) is routinely used as first serological marker in hepatitis B Virus (HBV) infection. The persistence of HBsAg beyond 6 months defines chronic hepatitis $\mathrm{B}(\mathrm{CHB})$ infection. In spite of the emerging importance of chemiluminescence based quantitative measurement of HBsAg for therapeutic monitoring of the patients, we tried to measure HBsAg concentration by sandwich ELISA in treatment nave subjects of $\mathrm{CHB}$ infection.

Methods: Sixty two subjects were evaluated for serum $\mathrm{HBeAg}$,anti HBe and ALT status by standard ELISA and biochemical procedures.Serum HBV DNA level was determined by real time TaqMan PCR assay (Roche 
Diagnostics,USA).Serum HBsAg level was measured by a third generation sandwich ELISA kit (Alpha Diagnostics International, USA).

Results: The median age of the subjects was 40.5 years (IQR $=18 ; 90 \%$ male) of which $92 \%$ were $\mathrm{HBeAg}$ negative and anti-HBe positive.Median ALT value was $35.5 \mathrm{IU} / \mathrm{ml}$ (IQR =37).Median viral load ( $\log$ copies $/ \mathrm{ml})$ and $\mathrm{HBsAg}$ $(\mathrm{IU} / \mathrm{ml})$ were $4.57(\mathrm{IQR}=2.84)$ and $38002.3(\mathrm{IQR}=23736.8)$ respectively.When grouped on the basis of viral load (), the median HBsAg concentration seen is 35439.3 (IQR= 43093.3) and 44712.7 (IQR=25082.4) while median ALT values were $29(\mathrm{IQR}=21.2)$ and $47(\mathrm{IQR}=73.2)$ in lower (2000 IU/ml)viral load groups respectively.

Conclusion: Serum HBsAg concentration showed no correlation with serum HBV DNA level in our study subjects which is at par with similar studies performed by chemiluminescence assay.Based on the reported correlation between serum HBsAg level and intrahepatic ccc DNA, higher amount of HBsAg in subjects having lower viral load is indicative of the presence of higher amount of intrahepatic HBV DNA in these subjects warranting further study.

\section{LV12}

\section{Reduction of circulating cytotoxic but not helper $\mathrm{T}$ cell} numbers in chronic hepatitis $B$ virus infection

P Balkumar Reddy, ${ }^{1} R$ M Mukherjee, ${ }^{\text {I }}$ Jyothi Arava, ${ }^{1}$ P N Rao, ${ }^{2}$ M Sasikala, ${ }^{l} R$ Gupta. ${ }^{2}$

${ }^{1}$ Asian Health Care Foundation, ${ }^{2}$ Asian Institute of Gastroenterology, Somajiguda, Hyderabad 500 082, India

Introduction: Patients with chronic hepatitis B (CHB) infection are often exhibit poor virus specific immune responses. Strong cytotoxic T lymphocyte (CTL) responses have been shown in acute hepatitis B virus (HBV) infection against the epitopes in $\mathrm{HBcAg}$ and $\mathrm{HBsAg}$. Since the persistence of $\mathrm{HBV}$ in human is related to the number and functional disorder of cell mediated immunity (CMI), the present study was undertaken to compare the peripheral immune cell profile of $\mathrm{CHB}$ patients including inactive carriers of HBV with healthy control subjects.

Methods: Fifty (29=HbsAg+Ve and 21=healthy controls) subjects were included in the study. Serum $\mathrm{HBeAg}$, antiHBe,ALT and AST were measured by ELISA and biochemical procedures.HBV DNA quantified through RealTime PCR assay. CD3+, CD19+, CD16-56+, CD4+ and CD8+ lymphocytes were enumerated using flow cytometry. Results: Among $29 \mathrm{HBsAg}+\mathrm{ve}$ subjects, $6.45 \%$ were positive for HbeAg and negative for anti Hbe. Significant decrease in Total T cell $(p=0.02)$ and Tc cell population $(p=0.018)$ observed in patients.In comparison to controls, significant decrease $(P=0.02)$ inT cell population encountered in inactive carriers $(n=10)$ than CHB patients $(n=19)$. Significant differences were found in $\operatorname{HBV}$ DNA $(P=$ $0.023)$, ALT $(P=0.01)$ and AST levels $(P=0.0112)$ but not with immune cells between inactive carrier and $\mathrm{CHB}$ patients.

Conclusion: In spite of the presence of high viral load and ALT, serum HBV DNA appeared as poor inducer of peripheral lymphocytes in $\mathrm{CHB}$ infection. The quantity of CD4+ T cells and CD4+/CD8+ ratio in peripheral blood of such patients were found to be significantly lower favoring viral persistence thus chronicity of the disease.

\section{LV13}

Risk factors for hepatocellular carcinoma in India with respect to $\mathrm{HBV}$ and $\mathrm{HCV}$ infection, aflatoxin content and alcohol consumption: A prospective study

\author{
Manash Pratim Sarma, ${ }^{1}$ Mohammad Asim, ${ }^{1}$ Subhash Medhi, ${ }^{1}$ \\ Premashis Kar. ${ }^{1}$ \\ ${ }^{1}$ Maulana Azad Medical College, New Delhi 110002
}

Aim: To assess the role of hepatitis B virus (HBV), hepatitis $\mathrm{C}$ virus (HCV), alcohol consumption and aflatoxin as risk factors for development of hepatocellular carcinoma (HCC) in India.

Methods: Three hundred and thirty-one patients with HCC and 120 control subjects without any hepatotropic viral infection were included in the study. Odds ratios (ORs) and $\mathrm{p}$ values were calculated separately for individual risk factors and in combination.

Results: The risk factors analysed for $\mathrm{HBV}$ and $\mathrm{HCV}$ markers of $\mathrm{HCC}$ with respect to control with $95 \%$ CI were 27.30 (12.81-60.12) were 17.96 (8.16-41.12) for HBsAg positivity, 17.29 (2.52-341.99) for HBsAg negativity and antibody positivity, 6.29 (1.84-25.88) for any HCV marker, 7.43 (1.71-45.28) for both anti HCV and HCV RNA positivity, $3.33(0.43-70.84)$ for anti HCV positivity and RNA negativity. In alcoholic group OR was 1.93 (0.993.86). No significance was found for anti $\mathrm{HCV}$ positive and RNA negative $(p=0.22)$ and with heavy alcohol intake $(p=.04)$. An overall risk of 1.21 was observed in the HCC patients with respect to AFB 1 exposure in food and 1.34 in urine. In aflatoxin positive cases, $\mathrm{HBV}(\mathrm{OR}=1.27)$ seems to be the only risk factor in food where as in urine HBV $(\mathrm{OR}=1.52)$, Alcohol $(\mathrm{OR}=1.95)$ and $\mathrm{NBNC}(\mathrm{OR}=3.27)$ were the possible risk factors.

Conclusion: In Indians $\mathrm{HBV}$ and $\mathrm{HCV}$ are the major risk factors for the development of HCC. HBV is significant risk factor alone whereas HCV in absence of HCV RNA is not a risk. Furthermore, HCV with heavy alcohol intake is a major risk factor. 


\section{LV14}

\section{Evaluation of Anti-HBs levels of medical students at a Medical College in coastal Orissa}

Shivaram Prasad Singh, ${ }^{\#}$ Chittaranjan Panda, ${ }^{\#}$ Haribhakti Seba Das, ${ }^{\#}$ Bijay Misra, ${ }^{\#}$ Swati Chouhan.

${ }^{\#}$ Hepatitis Research Centre, Department of Gastroenterology, S C B Medical College, Cuttack 753 007, India, *Kalinga Gastroenterology Foundation, S C B Medical College, Cuttack 753 001, India

Background and Objectives: All Health care workers and medical students should be vaccinated for Hepatitis B. Besides, after vaccination, the Anti-HBs levels must be checked to assess efficacy of the vaccine and seroprotection. The present study was conducted to evaluate the levels of Anti-HBs in medical students of a medical college in Orissa. Methods: Ninety medical students who participated in an awareness March on World Hepatitis Day were enrolled. The serum samples were evaluated for presence and titres of anti-HBs using commercially available enzyme linked immunosorbent assay (ELISA) kits.

Results: The mean age of the students was $22.39 \pm 2.0$ years; all except three were males. While all students had Anti-HBs levels above $10 \mathrm{IU} / \mathrm{L}$, only 35 of 90 [38.9\%] students had the desired level above $100 \mathrm{IU} / \mathrm{L}$. None of them had checked their Anti-HBs earlier. The common reasons for not testing for AntiHBs were lack of awareness about the necessity of such testing and ignorance regarding availability of testing facilities.

Interpretation and Conclusion: Only about one third of the medical students had desirable Anti-HBs levels of $>100 \mathrm{IU} /$ $\mathrm{L}$; the remaining two thirds might be at risk of developing HBV infection as a result of exposure. However due to the lag period between vaccination and testing, the low levels might not be a true indication of lack of seroprotection. All medical students should be tested for Anti-HBs levels after hepatitis B vaccination. Students with suboptimal Anti-HBs levels should be retested for seroprotection after a booster dose of hepatitis B vaccine.

\section{LV15}

\section{Adipocytokines and Steatosis in chronic hepatitis B and $\mathrm{C}$}

Sheetalnath B Rooge, G T Kumar, S S Hissar, A Rastogi, A Kumar, A Singh, $R$ Gondal, $S$ K Sarin, P Sakhuja.

G B Pant Hospital, Institute of Liver and Billiary Sciences, Delhi

Introduction: Adipocytokines are adipose tissue-derived secretary proteins. These may be related to the steatosis seen in chronic hepatitis and NASH. We aimed to assess the prevalence of steatosis and its relationship with biochemical, metabolic, and histologic parameters in patients with $\mathrm{CHB}, \mathrm{CHC}$ and NASH. We also determined adipocytokine levels and their correlation with above parameters.

Methods: Patients of CHB (114), CHC (63), NASH (30), and healthy controls (15) were included in the study. ELISA for Adiponectin $(n=81)$, Leptin $(n=99)$, Resistin $(n=101)$ and IL-6 $(n=101)$ was randomly performed in all the groups.

Results: Steatosis percent was greatest in NASH followed by $\mathrm{HCV}$, then $\mathrm{HBV}$ and was significantly higher with raised BMI $\left(>23 \mathrm{Kg} / \mathrm{m}^{2}\right)$ in CHB $(p=0.004)$ but not in CHC. The histologic activity and fibrosis scores were significantly higher in $\mathrm{CHC}$ than $\mathrm{CHB}$ and correlated with higher steatosis percent. CHB - patients showed increased serum Leptin $(p=0.036)$, IL-6 $(p=0.001)$ and adiponectin $(p=0.001)$ levels with high BMI $\left(>23 \mathrm{Kg} / \mathrm{m}^{2}\right)$. IL-6 levels correlated with raised steatosis $(p=0.01)$ and raised TG (0.037). CHC - 70\% of patients had genotype 3. The Adipocytokines did not correlate with BMI. Serum resistin levels showed significant correlation with raised SAP $(P=0.034)$ while raised IL-6 levels correlated with high SAP $(0.028)$ and fibrosis $(\mathrm{F}=3-6)$ $(P=0.002)$.

Conclusion: Steatosis is seen in $\mathrm{CHB}$ and $\mathrm{CHC}$ and correlates with increased histologic activity and fibrosis. In $\mathrm{CHB}$ but not in $\mathrm{CHC}$, steatosis was related to host metabolic factors. Altered adipocytokine levels influenced histologic activity and fibrosis in chronic viral hepatitis.

\section{LV16}

\section{Hepatitis A immunity in patients with cirrhosis of the liver}

R Anoop, Rony Thomas, Shibi Mathew, Pradeep Kumar, Selwyn Noronha, Premalatha, K $R$ Vinayakumar.

Department of Medical Gastroenterology, Medical College, Thiruvananthapuram

Background: Hepatitis A (HAV) superinfection is one of the causes for decompensation in patients with cirrhosis. Aim of this study is to assess the seroprevalence of anti-HAV antibodies in our population with cirrhosis.

Methods: This descriptive analytical study included consecutive cases of cirrhotsis being followed up in the department of Medical Gastroenterology, Medical college, Thiruvananthapuram. To account for the low immune response in cirrhotic patients, a control population was identified from the first degree relatives of the patient. IgG anti HAV antibodies were done in the cirrhotic patients and the control group.

Results: There were 90 cases and 40 controls. The commonest etiologies for cirrhosis were Alcohol in 50 (55.55\%), HBV in 
$15(16.66 \%)$, autoimmune in $5(5.55 \%)$, HCV in $3(3.33 \%)$, cryptogenic in $15(16.66 \%)$ and Wilsons in $2(2.22 \%)$. The CHILD status of the patients were C-45 $(50 \%)$, B-30 (33.33\%), A-15 (16.66\%). 86 were tested positive for $\operatorname{IgG}$ anti HAV in case group and 39 in control group. The seroprevalence of HAV was $95.5 \%$ in the patients and 97.5\% in controls..Fischer Exact test was applied to find the significance of the difference between the case and control and $P$ value was 1 which was non significant.

Conclusion: Most of the patients with cirrhosis of the liver in the population studied were already exposed to HAV. There was no significant difference in the immunity status of the patient group compared with the control group. Routine vaccination against $\mathrm{HAV}$ is not required in our group of patients.

\section{LV17}

Elevated level of pretherapy circulatory interferon gamma in hepatitis $C$ virus infection

Puja Gangwal, ${ }^{1} B$ Budhapriya, ${ }^{1} P$ Balkimar Reddy, ${ }^{1}$ $R M$ Mukherjee, ${ }^{1} M$ Sasikala. ${ }^{1} P N$ Rao, ${ }^{2} R$ Gupta, ${ }^{2}$ D Nageshwar Reddy. ${ }^{2}$

${ }^{1}$ Asian Health Care Foundation, ${ }^{2}$ Asian Institute of Gastroenterology, Somajiguda, Hyderabad 500 082, India

Introduction: Pegylated interferon (IFN) and Ribavirin in combination are currently recommended treatment for hepatitis C Virus (HCV) infection where approximately $30-40 \%$ patients fail to respond. Although the reason for non-response is partly explained by viral genotypes, studies on the role of host factors are scanty. The aim of the present study is to investigate the basal level of interferon (IFN) $\alpha$ and $\gamma$ in serum of $\mathrm{HCV}$ infected subjects in comparison to healthy individuals.

Methods: Thirty eight untreated HCV infected subjects who attended The Asian Institute of Gastroenterology, Hyderabad and 20 voluntary blood donors as controls were included in the study. Out of $38 \mathrm{HCV}$ positive subjects, 8 were followed up to 4 th week of IFN treatment. IFN $\alpha$ and $\gamma$ level in serum were assessed by flow cytometry and serum HCV RNA was measured by a TaqMan based real time PCR assay (Roche Diagnostics, Germany) as per manufacturer's instructions.

Results: Compared to IFN $\alpha$, IFN $\gamma$ level decreased significantly $(p<0.05)$ with increasing viral load. Significantly high basal level of IFN $\gamma(p<0.05)$ was evident in $\mathrm{HCV}$ infected who showed rapid virological response (RVR) after 4th week of IFN therapy.

Conclusions: Significantly elevated level of IFN $\gamma$ but not IFN $\alpha$ observed in untreated HCV infected subjects as well as in IFN responders indicate the role of innate immunity in $\mathrm{HCV}$ infection as well as for achievement of IFN mediated therapeutic response.

\section{LV18}

Expression of IFN a, IRF 1 and IRF 2 genes in chronic $\mathrm{HCV}$ infected subjects

B Budhapriya, ${ }^{1}$ P Balkumar Reddy, ${ }^{1}$ Puja Gangwal, ${ }^{1}$ $R$ M Mukherjee, ${ }^{l} M$ Sasikala. ${ }^{1}$

${ }^{1}$ Asian Health Care Foundation, ${ }^{2}$ Asian Institute of Gastroenterology, Somajiguda, Hyderabad 500 082, India

Objectives: The Interferon Responsive Factor (IRF) gene family mediate the IFN signal cascades where IRF1and IRF2 acts as positive and negative regulators respectively. This study is aimed to investigate the basal level of expression of IFN \pm , IRF 1, IRF 2 genes in treatment nave $\mathrm{HCV}$ infected subjects.

Methods: Peripheral blood mononuclear cells (PBMCs) from $29(\mathrm{M}: \mathrm{F}=23: 5)$ anti $\mathrm{HCV}$ positive subjects who attended the Asian Institute of Gastroenterology, Hyderabad and 17 (M:F = 17:0)voluntary blood donors (controls) were isolated, counted and numbers adjusted to $\sim 106 \mathrm{cells} / \mathrm{ml}$.RNA was extracted byTrizol,reverse transcribed and subjected to PCR assay for visualization upon agarose gel electrophoresis. Semiquantitative densitometric analyses of band intensities were used to evaluate gene expressions. Serum HCV RNA was measured by TaqMan based real time PCR assay (Roche Diagnostics, Germany) as per manufacturer's instructions.

Results: The viral load (mean \pm SD) observed in patient group was $5.63 \pm 2.10 \log$ copies $/ \mathrm{ml}$. The overall integrated density values (pixels) of IFN A, IRF1 and IRF2 were $3.51 \pm 2.89$, $6.12 \pm 4.0$ and $5.288 \pm 3.717$ in patients in comparison to $4.57 \pm$ $2.08,2.49 \pm 2.47$ and $2.21 \pm 2.83$ in controls respectively. Both IRF1 and IRF2 increased significantly.

\section{LV19}

\section{Expression of Interferon alpha receptor (IFNAR) and signal transducers and activator transcription (STAT) genes in HCV infection}

\author{
Budhapriya Bansode, ${ }^{1}$ P Balkumar Reddy, ${ }^{1}$ Puj Gangwal, ${ }^{1}$ \\ R M Mukherjee. ${ }^{I}$ \\ ${ }^{1}$ Asian Health Care Foundation, ${ }^{2}$ Asian Institute of Gastro- \\ enterology, Somajiguda, Hyderabad 500 082, India
}

Objectives: Activity of Interferon is mediated upon binding to specific cellular receptors - IFNAR to trigger the JakSTAT signalling pathway inducing antiviral state in the cell. In hepatitis $C$ virus infection, about $40 \%$ of the subjects fail to respond to IFN therapy.The present study is aimed to investigate the basal level of IFNAR1 and STAT1 gene expressions in untreated $\mathrm{HCV}$ infected subjects in respect to their viral load. 
Method: Peripheral blood mononuclear cells (PBMCs) from 35 anti HCV positive subjects and 17 voluntary blood donors who attended The Asian Institute of Gastroenterology, Hyderabad were isolated by Ficoll Hypaque, counted and numbers adjusted to $\sim 106$ cells/ml.RNA was extracted by Trizol and subjected to reverse transcription and subsequent PCR assay for visualization upon agarose gelelectrophoresis. Semiquantitative densitometric analyses of band intensities were used to evaluate gene expressions. Serum HCV RNA was determined by TaqMan based RT-PCR.

Results: IFNAR1 expression was undetectable in $51.4 \%$ of HCV infected subjects while $20 \%$ of HCV infected subjects failed to express of STAT1 gene. The overall integrated density values (pixels) of IFNAR1 and STAT1 were $3.30 \pm$ 2.10 and $7.87 \pm 5.54$ in patients in comparison to $1.31 \pm 0.37$ and $6.69 \pm 3.58$ in controls respectively. Higher expressions of IFNAR1, STAT1 genes observed with increasing concentration of serum HCV RNA.

Conclusion: HCV induced gene suppression was found to be more pronounced on IFN receptor level than the IFN signaling pathway. The Higher expression of IFNAR1 and STAT1 genes with increasing viral load might be indicative of the potential of those subjects to respond to IFN therapy.

\section{LV20}

To determine the incidence of HCV seroconversion in patients with end-stage renal disease on regular maintenance haemodialysis

Bhupender Singh, ${ }^{*}$ Jasleen Kaur, ${ }^{* *}$ Parveen Malhotra, ${ }^{* * *}$ Sethu Babu, ${ }^{*}$ Dharmesh Kapoor. ${ }^{*}$

*Department of Gastroenterology and Hepatology, Global Hospitals, Hyderabad $500004,{ }^{* *}$ Department of Anesthesia, Nizam's institute of Medical Sciences, Hyderabad 500 085, **** Department of Medicine, Post Graduate Institute of Medical Sciences, Rohtak

Background: Patients with End- stage renal disease (ESRD) on maintenance haemodialysis (MHD) are at an increased risk of acquiring $\mathrm{HCV}$ infection. Data on prevalence and incidence of de novo HCV seroconversion among Indian MHD patients are scanty.

Aim: To determine the incidence of HCV seroconversion in patients of ESRD on regular MHD, at a tertiary care hospital in South India.

Methods: Patients who underwent regular MHD at our centre between 1st July 2008 to 30th June 2009 were enrolled and followed up as per protocol.

Results: A total of 63 eligible subjects were enrolled and followed up for a period of 6 months to one year. Mean age of study cohort was 46.3 years, mean CKD duration was
29.7 months, $79.4 \%$ of subjects were males and 11 had HCV seroconversion. Incidence rate of de novo $\mathrm{HCV}$ seroconversion was $11.58 \%$ and $\mathrm{HCV}$ genotype 3 was the most common genotype (81.8\%) detected. De novo HCV seroconversion was associated with significantly higher Breakthrough ALT (ALT at the time of HCV seroconversion) in the HCV seroconverted group than in the non-seroconverted group ( $\mathrm{p}$ value $=0.017$ ).

Conclusions: Incidence rate of de novo HCV seroconversion was high (11.58\%) and HCV genotype 3 was found to be the most common genotype infecting this study population. Serial monitoring of ALT activity in MHD subjects was helpful in detecting de novo HCV infection, confirming the prior recommendations that serial ALT activity be monitored regularly in HD patients to detect de novo $\mathrm{HCV}$ seroconversion.

\section{LV21}

\section{Key determinants to predict the response rates of hepatitis $\mathbf{C}$ virus infected patients treated with peg-interferon alfa $2 \mathrm{a}$}

C Madhavi, S Rahamathullah, Y Naresh, M N Khaja, A $K$ Aleem, M H Aejaz, C M Habibullah.

Centre for Liver Research and Diagnostics, Owaisi Hospital and Research Centre, Kanchanbagh, Hyderabad 500 058, India

A significant association between variations in amino acid sequences residues of $\mathrm{HCV}$ nonstructural 5A (NS5A) gene, ISDR and PePHD regions and response to interferon treatment has been proposed. In the present study changes in PePHD and ISDR region were considered in chronic Hepatitis $\mathrm{C}$ subjects underwent for INF therapy when compared with non-responder prototype J. Based on the viral load and clinical factors, 19 out of 30 patients were found to be complete responders. The pretreatment viral RNA load found to be between 5.10 and $6.20 \log 10 \mathrm{IU} / \mathrm{ml}$, which eventually fell to $2.11 \log 10 \mathrm{IU} / \mathrm{ml}$ after 24 weeks, whereas in the case of non-responders, the average was $5.12 \log 10 \mathrm{IU} / \mathrm{ml}$. In present study at most one or two mutations at in ISDR region didn't correlate with the response pattern. However majority of responders showed a change in amino acid Serine to Alanine at position 660 of PePHD. Mutation at position 661 (Glutamic acid to Aspartic acid) was also found to be associated with response (2 out of 3 samples). Interestingly, histidine (position 667) found to be present in three samples and might influence the response to interferon. Methionine at position 662 was observed in seven non responders. Some significant changes in residues were observed in the PePHD region, which could be helpful in understanding the molecular basis of resistance to therapy, suggesting mutations in viral genome might have a concerted effect on interferon response. 


\section{LV22}

To determine the incidence of HCV seroconversion in patients with end-stage renal disease on regular maintenance hemodialysis

Bhupender Singh, ${ }^{1}$ Jasleen Kaur, ${ }^{2}$ Parveen Malhotra, ${ }^{3}$ Sethu Babu, ${ }^{1}$ K S Nayak, ${ }^{l}$ Dharmesh Kapoor. ${ }^{I}$

${ }^{1}$ Department of Gastroenterology and Hepatology, Global Hospitals, Lakd-ka-pul, Hyderabad 500 004, ${ }^{2}$ Department of Anesthesia, Nizam's Institute of Medical Sciences, Panjagutta, Hyderabad 500 085, India, ${ }^{3}$ Department of Medicine, Postgraduate Institute of Medical Sciences, Rohtak

Background: Patients with End- stage renal disease (ESRD) on maintenance hemodialysis (MHD) are at an increased risk of acquiring HCV infection. Data on prevalence and incidence of de novo HCV seroconversion among Indian MHD patients are scanty.

Aim: To determine the incidence of HCV seroconversion in patients of ESRD on regular MHD, at a tertiary care hospital in South India.

Methods: Patients who underwent regular MHD at our centre between 1st July 2008 to 30th June 2009 were enrolled and followed up as per protocol.

Results: A total of 63 eligible subjects were enrolled and followed up for a period of 6 months to one year. Mean age of study cohort was 46.3 years, mean CKD duration was 29.7 months, $79.4 \%$ of subjects were males and 11 had HCV seroconversion. Incidence rate of de novo $\mathrm{HCV}$ seroconversion was $11.58 \%$ and $\mathrm{HCV}$ genotype 3 was the most common genotype $(81.8 \%)$ detected. De novo HCV seroconversion was associated with significantly higher 'Breakthrough ALT' (ALT at the time of HCV seroconversion) in the HCV seroconverted group than in the non-seroconverted group ( $\mathrm{p}$ value $=0.017$ ). Conclusions: Incidence rate of de novo HCV seroconversion was high (11.58\%) and HCV genotype 3 was found to be the most common genotype infecting this study population. Serial monitoring of ALT activity in MHD subjects was helpful in detecting de novo HCV infection, confirming the prior recommendations that serial ALT activity be monitored regularly in HD patients to detect de novo HCV seroconversion.

\section{LV23}

\section{Clinical and laboratory features of severe dengue hepatitis: Early predictors of fatality}

Rajiv Mehta, Ujjval Jariwala, Ketan Desai, Anil Patel, Kiran Shah, Karshan Nandania, Hardik Astik.

Department of Gastroenterology, Smt R B Shah Mahavir Super-Specialty Hospital, Surat
Objective: To determine the early predictors of fatality among patients with severe dengue hepatitis based on clinical and laboratory findings.

Method: Forty-two patients who had Dengue Fever (DF, diagnosed by either positive serology or positive PCR technique) along with acute severe hepatitis (ALT $\geq 10$ times upper limit of normal) from March 2009 to July 2010 were included in the study. Various clinical and laboratory parameters of these patients on the day of admission were studied to identify early predictors of fatality.

Results: Forty-two patients (16 males, 26 females) were enrolled, out of which, twenty-six patients $(62 \%)$ died. Univariate analysis showed that age less than 40 years $(p=$ $0.02)$, Acute kidney injury $(p=0.04)$, AST $>5000 \mathrm{IU} / \mathrm{L}(p=$ $0.03), \mathrm{LDH}>10000 \mathrm{IU} / \mathrm{L}(p<0.001)$, Prothrombin Time $>20$ sec. $(p=0.04)$ were independently associated with mortality. Multivariate logistic regressive analysis showed LDH $>10000 \mathrm{IU} / \mathrm{L}$ and AST $>5000 \mathrm{IU} / \mathrm{L}$ were associated mortality. Conclusion: Severe dengue hepatitis is associated with high mortality. A very high LDH and AST were early indicators of fatality.

\section{LV24}

\section{Seroprevalence of hepatitis $B$ and $C$ among blood donors and a profile of hepatitis $B$ seropositive blood donors}

Venkata Sandeep, N Srinivas, Akbar, Aditya, Girinadh, $P$ Murali Krishna.

Department of Gastroenterology, Andhra Medical College, Visakhapatnam

Background: Hepatitis B and C are global health problems, serological surveys of blood donors helps in estimating the prevalence of HBV infection among adults in general population. Socio demographic profile of seropositive blood donors gives epidemiological determinants of susceptibly and risk factor estimation gives dynamics of disease transmission. Evaluating knowledge of Hepatitis B helps streamlining educational methods.

Aims: To determine the prevalence of serological markers of Hepatitis B and Hepatitis C virus among blood donors using Blood bank records. To know the socio-demographic profile, risk factors and knowledge of disease and vaccination among Hepatitis B positive blood donors in Visakhapatnam using interviews.

Methods: This is a descriptive type of study. Serological data of blood donors between January 2009 and June 2009, obtained from five blood banks in Visakhapatnam, a medical hub situated in north coastal Andhra Pradesh (India) catering to people of surrounding three districts with a total population of about 8 million. Screening for HBsAg and Anti HCV antibodies was done using enzyme immunoassays (ELISA) and 
rapid ELISA techniques. Through simple random sampling 55 Hepatitis B positive blood donors were interviewed for socio demographic characteristics, risk factors and knowledge of hepatitis B after informed consent.

Results: Of the 23,677 blood donors (voluntary 14,086 and replacement 9,591) $502(2.12 \%)$ donors [239 (1.70\%) voluntary and $263(2.74 \%)$ replacement donors] tested positive for Hepatitis B and $58(0.24 \%)$ donors [24 (0.17\%) voluntary and $34(0.35 \%)$ replacement donors] tested positive for Hepatitis C. Mean age of Hepatitis B seropositives was 27.4 , with $30.9 \%$ from urban and $69.1 \%$ from rural areas, all are literate, $25(45.5 \%)$ are dependant financially, 10 (18.2\%) belong to low income group. 38 seropositives heard of Hepatitis B of which 9 (16.4\%) know it to be infectious with $3(5.5 \%)$ naming 4 routes of transmission. $5(9.1 \%)$ seropositives heard of complications of Hepatitis B, 26 (47.3\%) are aware of the existence of vaccine for Hepatitis B but only 7 $(12.7 \%)$ took vaccine. $6(10.9 \%)$ seropositives gave history of multiple sexual partners, 3 (5.4\%) had previous blood transfusions, 13 (23.6\%) had previous surgeries and 12 (21.8\%) had a relative at residence with jaundice in the past.

Discussion: Seroprevalence of hepatitis B is $2.12 \%$, which is in the range of published prevalence data of $1-13 \%$ but less than published average prevalence of $4.7 \%$ in India. Seroprevalence of hepatitis $\mathrm{C}$ is $0.24 \%$. Prevalence is more among replacement than voluntary donors, more in rural than urban donors. Majority of the donors have inadequate knowledge of hepatitis B disease and vaccination. $23.6 \%$ seropositives had history of surgeries and $69.1 \%$ are from rural background with poor sterilisation techniques, $21.8 \%$ had a family member at home with jaundice, which is in accordance with published data of transmission routes as unsterilized needles and horizontal transmission in childhood.

Limitations: Recall bias during interviews.

Conclusion: The findings suggest prevalence of viral hepatitis among blood donors and risk of susceptibility among recipients, hence a need for improving sensitivity of screening techniques. There is also a need for intimating blood donors if tested positive and proper counselling is required. High prevalence in rural areas and poor.

\section{LV25}

\section{Decreased frequency of effector memory $T$ cells and concomitant increase in $\mathrm{CD}^{+}$and $\mathrm{CD8}^{+} \mathrm{T}$ cell exhaustion enhances the immune dysfunction during HCV-HIV co-infection}

Banishree Saha ${ }^{1,2}$, Manish Chandra Chaudhary ${ }^{1,2}$, Syed Hissar ${ }^{2}$, Reinhold Schmidt ${ }^{3}$, Shiv Kumar Sarin ${ }^{l}$.

${ }^{1}$ Department of Research, Institute of Liver and Biliary Sciences, New Delhi-110070; ${ }^{2}$ Department of Gastroenterology, G B Pant Hospital, New Delhi 110 002; ${ }^{3}$ Department of Immunology and Rheumatology, Hannover Medical School, Germany
Background \& aims: HIV-HCV co-infection leads to attenuation of HCV-specific immune responses causing higher HCV viral loads, rapid liver disease progression \& fibrosis. As the clearance of $\mathrm{HCV}$ is associated with vigorous multi-specific $\mathrm{CD} 4^{+}$and $\mathrm{CD} 8^{+} \mathrm{T}$ cell response, a qualitative defect in these cells due to HIV infection could further compromise immunity. The possible roles of $\mathrm{T}$ cell memory $\&$ exhaustion markers during co-infection were investigated. Methods: 53, treatment naïve patients were included; HCVHIV co-infected (GroupI, $n=17$ ), HCV mono-infected (Gr. II, $n=16$ ), HIV mono-infected (Gr.III, $n=20$ ) \& healthy controls (Gr.IV, $n=17)$. PBMCs were isolated \& immunophenotyped by flowcytometry.

Results: HCV-HIV infected patients had decreased $\mathrm{CD}^{+}$\& increased $\mathrm{CD} 8^{+}$Tcell frequencies $\left({ }^{*} P<0.05\right.$ vs.control). Increased proportions of $\mathrm{CD}^{+} \& \mathrm{CD}^{+}$naïve cells \& central memory Tcells $(\mathrm{Tcm})$ but a significant reduction in effector memory Tcell (Tem) frequency was observed. Increased levels of terminally differentiated effector memory cells (Temra)\& Tcell exhaustion marker PD-1 were also noted in co-infection cases. Further, a decline in the expression of CD27 (costimulatory receptor required for generation \& maintenance of Tcell immunity) on CD4+T cells was observed as compared to HIV mono-infected patients $(P<0.05)$.

Conclusions: Dysregulation of the immune system following reduction in the Tem cell population $\&$ increase in naïve population during co-infection may contribute to reduced antiviral response. Increase in the Temra cells with enhanced expression of inhibitory markers suggests senescence of these cells with infection. A decrease in CD27 expression \& enhanced PD-1 levels during co-infection results in skewing of $\mathrm{T}$ cells towards exhaustion. Together, these findings provide insights into why the cell-mediated immune responses are diminished during HCV-HIV co-infection.

\section{Liver}

\section{L1}

Prevalence of tuberculosis in cirrhotic patients in Western India

Rajiv Baijal, H R Praveenkumar, Mayank Jain, Deepak Gupta, D N Amarapurkar.

Department of Gastroenterology, Western Railway Hospital and Bombay Hospital, Mumbai, India

Introduction: Tuberculosis is a common disease in India with a prevalence of 5.05 per 1000. Cirrhotic patients are predisposed to tuberculosis due to immune system dysregulation.

Aim: 1) to assess the prevalence of tuberculosis in cirrhotic patients in Western India. 2) to analyze the site of 
tuberculosis, age and sex distribution and outcome in these patients.

Methods: This is a retrospective study. All patients of cirrhosis admitted at our centre in past 5 years were enlisted. All patients who developed tuberculosis after the diagnosis of cirrhosis were included. Patients who had past history of tuberculosis were only included if they had never defaulted on treatment and had been declared cured of tuberculosis earlier. The included patients were analyzed on the basis of etiology of cirrhosis, age, sex, site of tuberculosis, treatment given and outcome.

Results: Out of 677 patients enlisted, fifty patients had active tuberculosis (46 males). Thirty of these patients had alcoholic cirrhosis, 14 had post viral cirrhosis and 6 had cirrhosis due to other etiologies. The sites of tuberculosis included-pulmonary (20), pleural (14), peritoneal (9), cervical lymph nodes (30, liver (2), intestines (1) and vertebra (1) Thirty two cases were confirmed by histopathology. In the remaining cases, corroborative evidence was assessed prior to starting treatment. Thirty five patients received full dose antituberculous drugs and the rest were given modified regimen. $90 \%$ of the patients improved. Four patients died due to cirrhosis related complications (mortality rate 80 per 1000).

Conclusion: 1) the prevalence of tuberculosis is 73.8 per 1000. 2) It is more common in males and alcoholic cirrhosis patients 3) Extra pulmonary tuberculosis is more frequent 4) Mortality is higher than in general population.

\section{L2}

\section{Study of etiological factors for cirrhosis}

Rajiv Baijal, H R Praveenkumar, Mayank Jain, Deepak Gupta, $D N$ Amarapurkar.

Department of Gastroenterology, Western Railway Hospital and Bombay Hospital, Mumbai, India

Aim: To study the etiological factors responsible for cirrhosis in patients presenting to a tertiary care centre in Western India.

Methods: It is a retrospective study. All patients diagnosed with cirrhosis from 2003-2008 were analyzed. All patients had undergone full work up, including complete history, viral markers, lipid profile, blood sugar levels, serological tests and liver biopsy whenever required. The data obtained was analyzed. Results: A total of 645 patients were enlisted.535. Four hundred and twenty five were male patients and 110 were females. The main etiological factors for cirrhosis in descending order were- alcohol (253), hepatitis B (124), hepatitis C (89), NASH (106), cryptogenic (37), Wilson's disease (22), autoimmune hepatitis (8), primary biliary cirrhosis (5), hemochromatosis (1) and combined viral infection and alcohol (10). Amongst the males, the etiological factors were alcohol (241), followed by hepatitis B (101), NASH (85) and hepatitis C (52) in that order. In females, the main factors were hepatitis C (37), hepatitis B (23), NASH (21), cryptogenic (19) and alcohol (12).

Conclusion: Alcohol is the commonest cause of cirrhosis at our centre followed by viral hepatitis and non alcoholic steatohepatitis.

\section{L3}

Overlap syndrome: autoimmune hepatitis-primary biliary cirrhosis-A case report

D Sasi Anand, A Chezhian, S Anul prakash, K Muthu Kumaram, $R$ Bala Murali, $S$ Chitra, G Ram Kumar, $T$ Pugazhendhi, $S$ Jeevan Kumar.

Department of Digestive Health and Diseases, Government Kilpauk Medical College, Chennai 600 010, India

Overlap syndrome presents with characteristics of AIH and PBC or PSC. AIH-PBC overlap has been reported in $10 \%$ of cases with AIH or PBC. AIH-PBC overlap patients usually have features of autoimmune hepatitis with positive Anti mitochondrial antibody (AMA) and histologic features of bile duct injury or loss. Here we report a 44-year-old female presented with fatigue, pruritus, ascites, jaundice, pedal edema and splenomegaly. of 2 months duration with past history of jaundice 1 year ago. She denied intake of hepatotoxic drugs. Investigations: $\mathrm{Hb} 9.7 \mathrm{~g} / \mathrm{dL}$; PT 16/12 s, T.Bil $12.8 \mathrm{mg} / \mathrm{dL}$ (D. Bil 9.6), SGOT/PT 312/260 IU/L, SAP 389 IU/L, T.PRO $6.2 \mathrm{~g} / \mathrm{dL}$ (ALB 2.8); Urea $33 \mathrm{mg} / \mathrm{dL}$, creatinine $0.7 \mathrm{mg} / \mathrm{dL}$. HIV, HBsAg, IgM anti-HAV and HCV antibody were negative. USG abdomen showed cirrhosis with splenomegaly and ascites. Anti-nuclear, anti LKM anti body (1:100) and AMA (M2 antigen) by immunoflourescence (1:100) were positive. Anti- smooth muscle antibody was negative. UGI scopy revealed Grade II esophageal varices and mild PHG. Liver biopsy revealed florid bile duct lesion with interface hepatitis. AIH-PBC overlap was diagnosed using Chazouilleres et al. criteria and she was started on UDCA $15 \mathrm{mg} / \mathrm{Kg} /$ day. On follow-up, the patient showed both clinical and biochemical improvement. We report this case for its rarity and its good response to UDCA alone.

Conclusion: The diagnosis of AIH-PBC overlap is considered on clinical presentation, biochemical, immunological data and liver histology. The clinical course and treatment depend mainly on predominant component of the disease either hepatitic or cholestatic pattern and it responds to steroid or UDCA respectively. Recommendations for the treatment of AIH-PBC overlap have not yet been standardized owing to the low prevalence of this autoimmune liver disease. Liver transplantation is regarded as the treatment of choice for end stage disease. 


\section{L4}

Role of glycyrrhizin (SNMC-stronger neominophagen C) in alcoholic hepatitis including alcoholic cirrhosis

\section{P Jignesh, A Agasti, A R Samarth, C Vashishtha, N Mukesh, $R$ Goyal, Prabha Sawant. \\ Department of Gastroenterology, Lokmanya Tilak Muncipal Medical College and General Hospital, Sion, Mumbai 400 022, India}

Background: Inspite of many indepth studies into etiopathogenesis of alcoholic hepatitis and alcoholic liver disease, a universally acceptable, safe and effective therapy still remains elusive. SNMC has anti inflammatory action, anti oxidative action and cell membrane stabilisation activity.

Aim: To assess the efficacy and safety of SNMC in alcoholic hepatitis.

Methods: 15 consecutive hospitalized patients of alcoholic hepatitis (elevated S.Bil. AST to ALT ratio >2) were enrolled. Patients with viral hepatitis (acute/chronic), acute liver failure, those with S.cre.>1.5, HE and CCF were excluded. Inj. SNMC $40 \mathrm{ml} /$ day for 2 weeks after baseline hematological and biochemical investigations including LFTs, PT/INR and repeated after therapy. Pentoxifylline was given as indicated. $20 \mathrm{ml}$ of SNMC contains monoammonium glycyrrhizinate of $53 \mathrm{mg}$ (glycyrrhizin $40 \mathrm{mg}$ ) as active ingredient.

Results: Out of 15 cases studied serum bilirubin improved in $9(60 \%)$, no improvement in $6(40 \%)$. Transaminases normalized in $3(20 \%)$, improvement $10(66.7 \%)$, no improvement 2 (13.3\%). Overall subjective improvement [nausea, vomiting and anorexia] and sense of well being in $75 \%$ of cases after 2 weeks therapy despite persistence of liver disease. Side effect-No life threatening ADR.

Conclusion: This small and short study showed initial good and safe response to SNMC therapy in acute alcoholic hepatitis. It is essential to study a larger randomized group of patients with controls before any definite conclusion.

\section{L5}

Comparasion of sensitivity and specificity of DF $>32$ vs. DF $>40$ (discriminant function) in predicting mortality at 3 month in patients of Alcoholic hepatitis

A K Agasti, M G Srinivas, P Jignesh, A Samarth, C Vashistha, Prabha Sawant.

Department of Gastroenterology, Lokmanya Tilak Muncipal Medical College and General Hospital, Sion, Mumbai 400 022, India

Aim: To compare sensitivity and specificity of DF $>32$ vs. $\mathrm{DF}>40$ (discriminant function) in predicting mortality at 3 month in patients of Alcoholic hepatitis.
Methods: Total 67 patients of alcoholic hepatitis were followed up to 3 months prospectively. Patients with active alcohol consumption, at least $40 \mathrm{gm} /$ day for last 1 month and serum bilirubin $>2 \mathrm{mg} / \mathrm{dl}$ with elevated enzyme were included in the study. Any non alcoholic cause of hepatitis, death within 48 hrs of admission and history of G I bleed in past 6 weeks were excluded. All patients received pentoxiphylline and high protein diet.

Results: Out of 67 patients 3 were lost to follow up. So 64 patients data were analysed. Mean age was $42.6 \pm 8.7 \mathrm{y}$. Mean alcohol consumption was $70 \pm 40 \mathrm{~g} /$ day. Mean duration was $12 \pm 7$ y. Jaundice was present in $62(93.7 \%)$, abdominal distention in $44(64.7 \%)$, abdominal pain in 31 $(48.4 \%)$, fever in $14(21.9 \%)$ and altered sensorium in $8(12.5 \%) .24$ patients died out of $64.46 .87 \%$ patients with $\mathrm{DF}>32$ died and $6.25 \%$ with $\mathrm{DF}<32$ died. The sensitivity and specificity of DF $>32$ in predicting mortality at 3 month was $88.2 \%$ and $63.8 \%$ respectively but when DF $>40$ was considered sensitivity remains the same but specificity increased to $78.7 \%$.

Conclusion: DF $>32$ is widely used in clinical practice to treat alcoholic hepatitis patients with steroids. It has high sensitivity but low specificity. In our data, $\mathrm{DF}>40$ improved specificity (78.7 vs 63.8 ) without losing sensitivity.

\section{L6}

\section{Acute Budd-Chiari syndrome- Report of two cases}

K Kalyan, S Rajanikanth, Shanthi Vijayaraghavan, Shanmuganathan, Bavanarayana, Somasekar Rao, Rupesh, Seetharam, Naveen.

Sri Ramachandra Medical College, Porur, Chennai 600 116, India

Acute Budd-Chiari Syndrome is a rare entity. Two cases have presented to our institute between January 2010 and June 2010. First case is a 31 year old female patient, G3A2, at 31 weeks gestation with IUD, for which the dead fetus was expelled by an Obstetrician, was referred to us with severe epigastric pain. Patient had history of recurrent spontaneous abortions and was diagnosed with antiphospholipid antibody syndrome. Abdominal examination showed hepatomegaly and ascites. LFT showed T.B 2, D. B 1.4, SGOT 380, SGPT 170, Albumin 1.6, Globulin 3.1. INR was 1.7. Doppler study showed absent flow in the Hepatic Veins. She was started on LMWH and Warfarin. MRI abdomen was done 1 week after anticoagulation and did not show any thrombus in the Hepatic veins. She was discharged with warfarin. Second case was a 19 year old female patient who presented with acute hepatic failure. LFT showed T.B 4.09, D.B 2.75, SGOT 1298, SGPT 758, albumin 2. INR was 
4.08.S.Ammonia is 27.Viral markers were negative.Copper studies were negative. Doppler study showed no flow in the hepatic veins. Procoagulant work up showed low value of protein $\mathrm{S}$. factor $\mathrm{V}$ leiden, prothrombin gene mutation, protein $\mathrm{C}$, antiphospholipid antibodies, antithrombin levels were normal. In BCS liver disease makes it extremely difficult to distinguish primary deficiency in protein $\mathrm{S}$, from low plasma levels secondary to decreased hepatic synthetic function. She was started on LMWH. Liver transplantation was contemplated but the patient was lost to follow up.

\section{L7}

\section{Benign or malignant / rarer but deadlier tumor}

M Malarvizhi, Kani Shaikh Mohammed, B Vinoth, Biggs Saravanan, $K$ Premkumar, $K$ Caroline Selvi, $P$ Ganesh, P Padmanabhan, Mohammed Ali.

Department of Medical Gastroenterology, Madras Medical College, Chennai 600 003, India

Introduction: Hemangioma of liver and spleen are very rare benign tumors.Generally they are asymptomatic but large ones can cause mass effect, may obstruct extra hepatic portal vein causing portal hypertension, consumptive coagulopathy (kasabach meritt syndrome). Malignant transformation is a very rare phenomenon.

Case summary: $25 \mathrm{yr}$ female presented with abdominal pain and abdominal distention for2 yrs. Pain was severe, intermittent and relieved only with narcotics. She had myalgia and easy fatigability. Examination revealed massive splenomegaly and hepatomegaly. Investigations showed neutropenia, low platelets $(60,000)$ and prolonged PTINR (3.1). Hemoglobin was $4.1 \mathrm{~g} / \mathrm{dl}$. Hemoglobin electrophoresis showed normal adult pattern. Bone marrow biopsy showed a hyper cellular marrow with megaloblastic changes. USG abdomen showed multiple cystic lesion in liver with specks of calcification of spleen suggestive of cystic lymphangiomatosis. CECT abdomen showed multiple hypo echoic lesion in left lobe of liver and specks of calcification in spleen. Patients was managed with FFP transfusion and blood transfusion and hemanitics. In view of hypersplenism patient was subjected to open laparotomy. Spleen and left lobe of liver was resected. The right lobe of the liver was normal. Histopathology slides showed cavernous hemangioma of spleen and angiosarcoma of the right lobe of liver.

Conclusion: Hemangiomas are very rare benign tumors. Malignant transformation into angiosarcomas are very rare. Treatment option are limited due to rapid metastatic spread. Justification of surgery involves analysis of risk and benefit. The prognosis of angiosarcoma is very poor with a 5 yr survival less than $20 \%$.

\section{L8}

A study on environmental and genetic interaction towards the cancer predisposition and susceptibility in Manipur population

Rajkumari Deblakshmi, Manab Deka, Moumita Bose, Bharati Baruah, Manashree Sharma, Sujoy Bose.

Deparment of Biotechnology, Gauhati University, Guwahati, Assam

Molecular epidemiology of cancer predisposition (including those of hepatobiliary origin) in Manipur is understudied. Aim: Investigate role of Cyp2E1 genotype and environmental factors (including dietary habits, addictions, etc.) towards cancer predisposition in Manipur.

Methods: Physically healthy $(n=55)$ population were enrolled and stratified into four etiological groups viz; groupI $=$ controls $(n=10), \mathrm{II}=$ alcoholic group $(n=10), \mathrm{III}=$ tobacco user $(n=20)$, and IV $=$ alcoholic tobacco user $(n=15)$ according to their habits. Few cases $(n=10)$ cases has hepatobiliary cancer history in their family. Polymorphism in CYP2E1*5B was examined by PCR-RFLP. Nitrite concentration in the indigenous fermented and raw food stuffs were determined using Greiss's reagent. Results: Although the prevalence of mutant genotype $\mathrm{C} 1 / \mathrm{C} 2$ was higher in the control group, the genotype distribution was not found significant amongst the studied groups $(p=N S)$. No statistically-significant difference in CYPE1 genotype distribution was noticed between cases with and without family history of hepatobiliary cancer. The presence of $\mathrm{C} 1 / \mathrm{C} 1$ wild type genotype increased the risk of hepatobiliary disease caused by the etiological agents studied in the present study. There was a high percentage of nitrites concentration in the fermented food samples compared to raw ones.

Conclusion: Statistically, CYP2E1*5B C1/C1 genotype may be associated with higher risk for the above studied etiological agents mechanized cancer development. Presence of high nitrite concentration is alarming, as nitrite are pre-carcinogens which can be converted to carcinogens by the action of phase 1 Cyp2E1 enzymes and contribute towards cancer susceptibility.

\section{L9}

\section{Post liver transplant biliary complication - Case series}

M Agarwala, Bipin, M Barath, T Amrutesh, A Kakkar, A Ramamurthy, KR Palaniswamy, U Dhus.

Department of Gastroenterology, Apollo Hospital, Chennai

Introduction: Biliary complications occurs in 5-25\% of patients following liver transplantation. These complications includes, biliary stricture, bile leak, choledocholithiasis, can occur in both living as well as cadaveric liver transplantation 
Method: Here we presented 8 patients who developed biliary complication following liver transplantation in our center.

Result: Out of 61 liver transplantation (12 living and 49 cadaveric donor) 8 patients $(13.11 \%)$ developed biliary complication $(25 \%$ in living donor, $10.2 \%$ in cadaveric group). Bile leak was the most common biliary complication (7 out of 8 patients). 3 patients developed biliary stricture, 1 patient stricture alone and 2 patients following initial bile leak. Time of bile leak, following LT ranges from 15-40 days in 7 patients, and 3 months later in 1 patient with an initial attack of HAT. 1 patient developed early biliary stricture within 2 weeks of surgery, and 2 patients developed delayed stricture. CMV infection was found in 3 patients with bile leak. Diagnosis was made on the basis of clinical suspicion, USG abdomen HIDA scan and ERCP according to situation. 6 patients could be managed by ERCP and stenting alone, 2 patients required surgical intervention. 2 patients died of sepsis.

Conclusion: Biliary complication is a known complication following LT (13.11\% in our center).Bile leak and stricture are two important complication.Most of these cases can be managed by ERCP, some may require surgery.

\section{L10}

\section{An interesting case of hepatomegaly}

B Prakash Shankar, P Jagadesan, G Ramkumar, K Muthukumaran, T Pugazhendhi, S Jeevan Kumar.

Department of Digestive Health and Diseases, Government Peripheral Hospital, Chennai 600 010, India

Introduction: Hemangioma of liver is the most common benign space occupying lesion of liver. The clinical importance of hemangioma is to differentiate this condition from more serious disorders.

Case Report: Forty years old female presented with c/o Rt. Upper quadrant pain and fullness for six months duration. Examination revealed a firm, smooth, non tender hepatomegaly with a liver span of $23 \mathrm{cms}$ without bruit. Baseline investigations were normal, $\mathrm{RBC}$ and $\mathrm{WBC}$ counts with a low normal platelet count. LFT and RFT were normal. OGD was normal. USG showed a massive hyper echogenic liver mass measuring $25 \times$ $24 \mathrm{cms}$, with satellite lesion in right lobe. CECT Abdomen showed $26 \times 26 \mathrm{cms}$ large hypodense mass lesion involving both lobes of liver with compressed normal parenchyma posteriorly. Lesion showed nodular vascular like enhancement with filling in from periphery. Satellite lesion present. MRI Abdomen showed hypointense mass in T1 weighted images with markedly hyperintense homgenous pattern in T2 image characteristic of hemangioma. Patient was given reassurance and follow up for a period of 6 months was uneventful.

Discussion: Radiological procedures play a primary role in diagnosing hemangioma. MRI is the most sensitive and specific means of diagnosing hemangioma. Percutaneous biopsy, which carries some risk of hemorrhage, is rarely required.

Conclusion: Hemangioma is the most common benign space occupying lesion of liver. It can present in varying sizes some of them as large as $26 \mathrm{~cm}$. Surgical excision is necessary only if symptomatic.

\section{L11}

Clinical spectrum and prognostic factors of acute liver failure in North Kerala

S Shareej, M Iqbal, K Sunil Kumar, T M Ramachandran, Varghese Thomas $M$.

Department of Gastroenterology and Internal Medicine, Medical College Calicut, Kerala

Objective: To analyze the demographic profile, etiology and disease characteristics and prognostic variables of ALF presenting to a tertiary care centre.

Methods: The details of patients who presented to the department of Gastroenterology and Medicine of Calicut Medical College between January 2010 and July 2010 are collected prospectively in a structured proforma. Routine laboratory tests, hepatitis serology, arterial ammonia, alfa fetoprotein, autoimmune markers and copper profile were done in all.

Results: 17 patients were seen during this period. The F:M ratio was 1.1:1 with a mean age of 26.59 with SD of \pm 9.99 years. 9 $(52.9 \%)$ patients survived while $8(47.1 \%)$ died. Etiology of ALF was identified in 14 cases such as acute hepatitis A in 7, rat poison injestion in 3, AFLP in1, HELLP syndrome in1, ATT in1 and 1case was diagnosed as sickle disease by postmortem liver biopsy. 9 patient developed features of encephalopathy in first 7 days, 3 during 8 â $\epsilon^{\text {" }} 14$ days, 1 during $14-21$ days and 2 during 21-28 days. Total bilirubin level was high in those who died (20.20 with a SD of \pm 8.915$)$ compared to $9.2 \pm 5.308(P=.008)$ those who survived. Prothrombin time was prolonged in those who died (mean 40.363 with SD $25.623 t$ test $3.300 p=.005$ ). Level of AFP at day 1 and 3 indicated better prognosis.

Conclusions: The main cause of ALF in our centre is viral hepatitis followed by rat poison. High bilirubin, prothrombin time and INR predicted worse outcome. Higher AFP level showed a better survival chance.

\section{L12}

Clinical profile of hepatocellular carcinoma in a tertiary care centre in North Kerala

S Shareej, Ashik Sainu, K Sunil Kumar, T M Ramachandran, Varghese Thomas, Ajay Kumar.

Departments of Gastroenterology and Radiotherapy, Calicut Medical College, Kozhikode, Kerala 
Objective: To analyze the clinical profile and disease characteristics of hepatocellular carcinoma (HCC) presenting to a tertiary care centre in north Kerala.

Methods: The case records of patients with HCC between Jan 2007-Dec 2009 were retrieved and disease characteristics were analyzed as per a structured proforma. Only patients with imaging and/or histopathologic evidence of HCC were included in the study.

Results: A total of 101 patient records were reviewed. The M: $\mathrm{F}$ ratio was $6.75: 1$ with a mean age of 59.31 with SD of \pm 11.84 years. $42.6 \% \%$ had established cirrhosis of the liver with chronic hepatitis B being the most common etiologic agent $(28.7 \%)$ closely followed by alcohol (27.7\%). There was a significant difference in the number of patients presenting with ascites among patients with cirrhosis (66\%) liver compared to the noncirrhotics $(41 \%)(p<0.005)$. Diagnostic level $(>400 \mathrm{ng} / \mathrm{ml})$ of serum AFP was seen in $52.5 \%$ of patients with no significant difference between patients with and without cirrhosis $(p=0.5)$.Confirmation of $\mathrm{HCC}$ by FNAC was possible in $61(60.4 \%)$ cases. Portal vein invasion was seen in $28.7 \%$ patients. The average size of the lesion on presentation was $6.24 \pm 2.04 \mathrm{~cm}$. Apparently resectable HCC as per the clinical records were 8 cases (7.92\%).

Conclusions: The clinical presentation of HCC in our centre is quite consistent with data from the north India. Number of cirrhotics with HCC in our group was high. $92 \%$ of patients with HCC presented with advanced disease when only option is palliation.

\section{L13}

\section{Are liver and pancreas friends or foes in alcoholics?}

V Arulselvan, B Sivasubramaniam, V R Vimalraj, Surendran, V Jayanthi, Arunkumar.

Department of Gastroenterology, Stanley Medical College, Chennai 600 003, Tamil Nadu, India

Background and Aim: This study compares the similarities and dissimilarities between alcohol related pancreatitis and liver disease with an intention to identify risk factors predisposing the individual to one disease but not the other. Methods: Consecutive patients (201) attending the Department of Gastroenterology both inpatient and outpatient and presenting with features of alcoholic pancreatitis and/or liver disease between January 2010 and July 15, 2010 were included for the study.

Results: There were 61 patients in pancreatitis (Group-I), 97 with liver disease (Group- II), 11 had both liver and pancreas (Group-III), 32 in Control (group IV) group. The mean age of presentation in the 4 groups were 37, 44.6, $45 \& 42$ years respectively. The socioeconomic status, literacy, occupation and religion were similar in all the 4 groups so also was the smoking pattern. Current alcohol consumption was more in group I $(n=44 / 61)$. The mean duration of alcohol consumption was $13.1,16.5,15.4$, and 12.5 years respectively. The average consumption of alcohol per week was more in groupII (765.3 g) compared to group I, III and group IV, (653 gms, $653 \mathrm{~g}$ and $464 \mathrm{~g}$ ). In the past alcohol consumers, the duration of consumption was longer (22 years). Average consumption was 690 gms, 795 gms, 501 gms and 317 gms respectively. Pan chewing, diet pattern was similar in all groups.

Conclusion: Alcohol-related pancreatitis developed at a much younger age than liver disease. The duration of alcohol consumption and quantity consumed per week was less in pancreatitis compared to alcohol related liver disease.

\section{L14}

\section{Bacteriological profile in spontaneous bacterial peritonitis}

Ratnagiri Bandaru, Nalini Prasad Ippela, J Sunil kumar, Ramanna Macherla, M Uma Devi, A Vidya Sagar.

Department of Gastroenterology, Gandhi Medical College/ Hospital, Secunderabad, A P

Background: Spontaneous bacterial Peritonitis is a serious and frequent complication in cirrhosis. Community acquired SBP cases were more frequent $(60 \%)$ compared to the nosocomial acquired SBP - $40 \%$.

Method: We have analysed prospectively 161 inpatients with cirrhosis and ascites between 2007-2009. All these patients were subjected to routine hematological investigations, USG abdomen, ascitic fluid analysis. Ascitic fluid was subjected to culture after bedside inoculation in blood cultre bottles and antibiotic sensitivity and treated with antibiotics and followed. Results: Out of 161 pts with COL and ascites, 102 were males, 59 were females. 71 pts were clinically suspected SBP, in which $43(60.5 \%)$ became culture positive and in rest who were not clinically suspected SBP only 7 (7.7\%) became culture positive. Out of $71 \mathrm{pts}, 14$ were nosocomial and rest were community acquired.Of 50 pts, $78 \%$ were gram -ve and $22 \%$ were gram + ve. E.coli was the most common organisms $(80 \%)$, Klebsiella $(15 \%)$ pneumonia and Enterobacter (5\%) Staph. aureus was the most common among $\mathrm{Gr}+\mathrm{ve}(90 \%)$. Most of the Gr-ve bacilli were sensitive to cefotaxime (75\%), ofloxacin (20\%), amoxicillin clavulanic acid (5\%) Community Acquired Gr-ve organisms were more sensitive to Cefotaxime than nosocomial ( $80 \%$ vs. $75 \%$ ). $\mathrm{Gr}+\mathrm{ve}$ bacteria were more sensitive to cefotaxime (45\%) amoxycillin clavulanate $33 \%$, Ceftrioxone- $12 \%$. Complications like liverfailure $(10 \% \mathrm{vs} 10 \%)$, renal failure $(30 \%$ vs $16.6 \%)$, hepatic encephalopathy $(20 \% \mathrm{vs} 13 \%)$, gastro intestinal bleed (25\%vs16\%) were more common with nosocomial SBP than community acquired SBP. The mortality rate at 10 days was more with nosocomial. (30\% vs $16 \%$ ). 
Conclusions: complications and mortality were more common in nosocomial SBP. cefotaxime was found effective in both the groups.

\section{L15}

Vitamin $B_{12}$ deficiency and hyper homocysteinemia in non alcoholic fatty liver disease

K S S Uma Mahesh, ${ }^{1}$ A M Sadik, ${ }^{1}$ M Sasikala, ${ }^{1}$ G Deepika, ${ }^{2}$ R M Mukherjee, ${ }^{1}$ P N Rao. ${ }^{2}$

${ }^{1}$ Asian Health Care Foundation, ${ }^{2}$ Asian Institute of Gastroenterology, Somajiguda, Hyderabad 500 082, India

Introduction: The prevalence of obesity, Non alcoholic fatty liver disease (NAFLD), Diabetes and coronary artery disease (CAD) is increasing worldwide. Hyper homocysteinemia is implicated as a causative factor of endoplasmic reticulum stress, hepatic steatosis and endothelial cell damage leading to cardio vascular disease (CVD). Since vitamin $\mathrm{B}_{12}$ deficiency is one of the most important causative factor of hyper homocysteinemia, this study aims to observe serum levels of homocysteine and vitamin $\mathrm{B}_{12}$ in human NAFLD patients. Method: Patients diagnosed to have NAFLD by standard clinical, radiological and biochemical investigations formed the study group. Healthy volunteers formed the control group. Fasting blood samples were collected in the hepatology unit of Asian Institute of Gastroenterology and were subjected to biochemical analysis. Vitamin $\mathrm{B}_{12}$ and homocysteine were assayed using chemiluminiscence commercial kits. Liver function tests (LFT), lipid profile and HOMA IR were assayed using autoanalyser.

Result: Vitamin B12 deficiency $(<211$ picograms $/ \mathrm{mL})$, was observed in $43 \%$ of NAFLD patients and $72 \%$ of these vitamin B12 deficient patients had hyperhomocysteinemia ( $>16.2 \mathrm{micromols}$ ). Elevated ALT was found to be correlated with $67 \%$ of vitamin B12 and $70 \%$ of hyperhomocysteinemic NAFLD patients respectively.

Conclusion: The results obtained indicate that hyperhomocysteinemia may be one of the factors responsible for hepatic steatosis in NAFLD patients.

\section{L16}

Intra biliary rupture of hepatic hydatid cyst with obstructive jaundice-A Case report

S Babu Kumar, $R$ Senthil Kumar, S Chitra, R Bala Murali, $S$ Jeevan Kumar, T Pugazhendhi.

Department of Digestive Health and Diseases, Government Peripheral Hospital, Anna Nagar, Chennai 600102 and Kilpauk Medical College, Chennai 600 010, India

Fifty-eight year old female presented with dull aching pain in the RUQ for 3 months associated with intermittent fever with chills and jaundice for 10 days. Physical examination revealed minimal tenderness in RUQ with firm, non tender hepatomegaly. CT abdomen showed well differentiated abscess cavities with wall calcification largest one measuring $7.6 \times 5.8 \mathrm{cms}$ in segments VI \& VII. Investigations showed $\mathrm{Hb}$ of $8.5 \mathrm{gms} \%$, total bilirubin of 4.1 with direct of $1.9 \mathrm{mgs}$, SAP of $1360 \mathrm{IU}$, mildly elevated SGOT and SGPT with INR of 1.21. MRCP showed hydatid cyst in the right lobe of the liver, ruptured into the right hepatic duct causing dilated IHBR in both lobes of the liver. ERCP showed bulging ampulla with protruding cyst material. Biliary sphincterotomy was done. Lot of cyst material extruded. A $10 \mathrm{fr} \times 10 \mathrm{cms}$ double pigtail stent was placed with its proximal end in the cyst cavity. The patient was given 6 one month cycles of albendazole of $400 \mathrm{mg} 1 \mathrm{bd}$. Repeat USG after 3 months showed marked reduction in cyst size along with clinical recovery. The biliary stent was removed during the same visit. We report this case to stress ERCP with sphincterotomy has been successful as the safe and effective mode of treatment of intrabiliary ruptured hydatid cysts.

\section{L17}

\section{Evaluation of transaminase levels in medical students} at a medical college in coastal Orissa

Shivaram Prasad Singh, \# Chittaranjan Panda, \# Haribhakti Seba Das, " Bijay Misra, " Swati Chouhan."

"Hepatitis Research Centre, Department of Gastroenterology, S C B Medical College, Cuttack 753 007, India, 'Kalinga Gastroenterology Foundation, S C B Medical College, Cuttack 753 001, India

Background and Objectives: There is considerable controversy regarding what constitutes upper limit of normal [ULN] for transaminase levels. Studies across the globe have suggested a lower cut-off value for ULN for AST and ALT. The present study was conducted to evaluate the transaminase levels in the undergraduate medical students of a medical college in Orissa.

Methods: Ninety medical students who participated in the Hepatitis B awareness March on World Hepatitis Day 2010 were enrolled in this study. The serum samples obtained from these students were assayed for the transaminase [ALT and AST] levels in an autoanalyser using standard kit, and the presence of HBsAg was tested by using commercially available enzyme linked immunosorbent assay (ELISA) kits. Results: The mean age of the subjects was $22.39 \pm 2.0$ years; all except three were males. All were negative for HBsAg. The mean AST and ALT levels were 36.54 $\pm 13.64 \mathrm{IU} / \mathrm{L}$ and $37.24 \pm 21.46 \mathrm{IU} / \mathrm{L}$. Asymptomatic elevation AST and ALT levels $[>40 \mathrm{IU} / \mathrm{L}]$ were detected in 21 [23.3\%] and 29 [32.2\%] respectively. Overall, only 58 [64.4\%] had normal 
transaminase levels; elevation of AST and/or ALT was present in 32 [35.55\%]. All the medical students were healthy and none had sign/symptoms of liver disease.

Conclusions: The results from this limited sample survey suggests that there is perhaps no need for re-evaluation of the ULN for AST and ALT levels in this region. Besides, about one third of the medical students had asymptomatic transaminitis by current standards and need further evaluation for presence of metabolic syndrome and NAFLD.

\section{L18}

\section{Prevalence of autoantibodies in Indian patients with chronic liver disease}

\author{
Sunilbaran Daschakraborty, Pallavi Shukla, Amita Aggarwal, \\ *Rakesh Aggarwal. \\ Departments of Gastroenterology and Immunology, *Sanjay \\ Gandhi Postgraduate Institute of Medical Sciences, Lucknow \\ 226014 , India
}

Background: Data on prevalence of autoantibodies in patients with chronic liver disease (CLD) show large inter-study variations. We studied the prevalence of autoantibodies in Indian patients with CLD, and their relationship with disease etiology and severity.

Methods: Sera from 146 (114 male, mean age: 45 years, cirrhosis: 120) patients with CLD (47 alcohol-related, 39 cryptogenic, $19 \mathrm{HCV}, 20 \mathrm{HBV}, 6 \mathrm{HCV}+\mathrm{HBV}, 4$ Budd-Chiari syndrome, 3 autoimmune, 1 secondary biliary cirrhosis, 3 Wilson disease) were tested for anti-nuclear antibody (ANA; 1/100), anti-smooth muscle antibody (SMA; 1:40), and anti-liver/kidney microsome type 1 antibody (anti-LKM1; 1:40) using indirect immunofluorescence.

Results: One or more autoantibodies were detected in 46/ 146 (31\%) patients, including 10/47 (21\%) with alcoholic CLD, 7/19 (36\%) with HCV-related CLD, 4/20 (20\%) with HBV-related CLD and 25/60 (40\%) of others. Autoantibody prevalence was higher in patients with HCV than in those due to alcohol or HBV disease $(36 \%$ v $21 \%$ and $20 \%$, respectively). Autoantibody prevalence rates were 20/75 (26\%), 15/37 (40\%) and 2/12 (16\%) in Child class C, B and A disease. ANA was detected in $21(14 \%)$ patients (alcohol 5, HCV 4, HBV 1) and ASMA in 33 (23\%) (7, 4 and 3 respectively). No patient had anti-LKM1. Using the same assays, we have previously found ANA, ASMA, anti-LKM or any of these in $4.3 \%, 6 \%$ and $0 \%$ of healthy persons, respectively.

Conclusions: Autoantibodies are frequent in patients with known cause of CLD, being higher in those due to HCV, than those due to alcohol or HBV.

\section{L19}

Frequency and pattern of impairment in health-related quality of life (HRQL) in liver cirrhosis

Prerna Singh, Preeti Srivastava, Divya Singh, Gourdas Choudhuri.

Department of Gastroenterology, Sanjay Gandhi Postgraduate Institute of Medical Sciences, Lucknow 226 014, India

Background and Aims: Liver Cirrhosis (LC) is a chronic progressive disease that affects health related quality of life (HRQL). We studied the frequency and pattern of HRQL impairment in different grades of LC.

Method: We evaluated the clinical, demographic and laboratory data of 495 patients of Liver Cirrhosis (male 381, mean age $45 \pm 10.9$, Child A: 142, Child B: 133, Child C: 109) and measured their HRQL by Short Form 36 and Nottingham health Profile questionnaires. Apart from noting the number who reported poor HRQL in each Child-Pugh class, we analysed the $8 \mathrm{HRQL}$ parameters that were most frequently affected in them.

Result: Poor HRQL was noted in 18\% (26/142) of patients with Child A, and increased progressively to $50 \%(66 / 133)$ in Child B and 87\% (95/109) in Child C patients. The most common parameters to be impaired were emotional well-being and social functioning Child A patients, whereas physical functioning and general health were the ones commonly impaired in Child B. Poor HRQL in Child C patients showed impairment of several parameters such as low energy, fatigue, pain, role limitation due to emotional problem and physical health. Pain was specifically observed in Child C.

Conclusions: HRQL is significantly affected in LC, the frequency increasing with the stage of disease. $18 \%$ of Child A cirrhotic has poor HRQL, largely due to emotional and social consequences. Loss of energy and fatigue were additional factors in Child B, whereas pain, physical weakness and role limitation were the main factors in patients with Child C.

\section{L20}

Predictors of hospital stay and mortality in patients with alcoholic hepatitis with discriminant factor $>32$

Rony Thomas, $R$ Anoop, Mahesh Varma, Praveen Kumar, Selwyn Norohna, Premalatha, $K R$ Vinayakumar.

Department of Medical Gastroenterology, Medical College Thiruvananthapuram, Kerala

Background: Alcoholic hepatitis and cirrhosis occur in 15$20 \%$ in alcoholics and has mortality $\sim 50 \%$ if $\mathrm{DF}$ is $>32$. Identifying individuals with high risk for mortality is 
paramount. Aim of present study was to identify any other factors other than MELD, DF that determine short term prognosis measured at time of hospital stay.

Methods: Study was conducted as a descriptive analytical study conducted in Department of Medical Gastroenterology, Medical College, Thiruvananthapuram. Study period was November 2008 to February 2010.

Inclusion Criteria: All patients who were diagnosed as Alcoholic hepatitis, as evidenced by elevation of AST more than ALT, history of alcohol intake $>80 \mathrm{gm} /$ day for males and $40 \mathrm{gm}$ for females and DF $>32$ were included in study. Demographic, clinical, biochemical parameters, USG abdomen, endoscopy were done on admission. MELD and DF scores were calculated.

Results: 125 patients fulfilled inclusion criteria. All were males.One month survival rate was $60.9 \%$. There were 49deaths (39.2\%).Most common cause of death was sepsis. Other causes of decreasing order of frequency were GI bleed, HRS and HCC.Apart from well established criterias like DF, MELD, the following parameters at admission were found to be significant predictors (pearson correlation and ROC curves were used) of in hospital mortality (1) Sodium $<128.5$ meq/lt (2) WBC count $>10080$ (3) ESR $>51 \mathrm{~mm} / \mathrm{hr}$ (4)Platelet count $<93500$.

Conclusions: This study revealed that apart from well established scoring systems like MELD, DF, the predictors of in hospital mortality in patients presenting with alcoholic hepatitis with DF $>32$ included low sodium, elevated WBC count, elevated ESR and low platelet count.

\section{L21}

Risk factors predicting mortality in cirrhosis of the liver

K Sridhar, V Jayanthi.

Medical Gastroenterology Department, Government Stanley Hospital, Chennai 600 003, India

Background: Cirrhosis of the liver is associated with structural and functional changes in liver leading to complications from portal hypertension and decreased hepatic function which cause morbidity and mortality associated with cirrhosis. Mortality increases with the severity of hepatocellular dysfunction and PHT.

Aim: To analyze the risk factors associated with mortality in patients with cirrhosis liver \& thereby predicting the prognosis \& mortality risk in patients presenting with cirrhosis liver.

Methods: Consecutive patients who expired due to cirrhosis after admission were included in this study. History, illness duration, CTP status, MELD, co-morbidities and the cause of mortality were studied.

Results: 61 patients were included in the study. 50 patients were male. Mean age is 45 years. The most common cause of cirrhosis is alcohol (47 patients). Of the 61 patients, 12 were CTP Child's A status while the rest were Child's B or C status. The cause of death in patients with Child's A status is uncontrolled UGI bleed. The causes of death in patients with Child's $\mathrm{B} / \mathrm{C}$ status is predominantly due to hepatic encephalopathy (33 patients). HRS accounted for 21 patients cause of mortality. UGI bleed was associated with mortality in 8 patients. All patients with cirrhosis B/C were associated with hyponatremia.

Conclusion: UGI bleed was associated with mortality in patients with Child A cirrhosis while the causes of cirrhosis in patients with child $\mathrm{B} / \mathrm{C}$ is due to hepatic encephalopathty and hepatorenal syndrome. Significant number of patients had hyponatremia.

\section{L22}

\section{Frequency of systolic and diastolic dysfunction} in patients with cirrhosis

Amit Goyal, Alok Sehgal, Nishant Tripathy, 'Samir Mohindra, Sudeep Kumar, *G Choudhuri.

Department of Gastroenterology and *Cardiology, Sanjay Gandhi Postgraduate Institute of Medical Sciences, Lucknow 226 014, India

Introduction: Cardiovascular abnormalities associated with cirrhosis are being increasingly recognised. There is scarce data regarding cardiac dysfunction in cirrhotics.

Methods: This prospective study was conducted in Departments of Gastroenterology and Cardiology, 70 cirrhotic patients and 20 controls were enrolled. The cardiac evaluation was done using ECG, echocardiography and serum brain natriuretic peptide.

Result: Median age of patients was 45 years (range 30-58) and $72 \%$ were male. Tissue Doppler imaging studying diastolic velocity across mitral septal annulus (E: E's ratio) (normal value $<8$ ) and mitral lateral annulus $\left(\mathrm{E}^{\prime} \mathrm{E}^{\prime} \mathrm{L}\right.$ ) (normal value $<6$ ), showed diastolic dysfunction in $64.2 \%$ cirrhotics as compared to $10 \%$ controls $(P<0.001)$. The median E deceleration time (ms) in cirrhotics and controls was $170(100-229)$ and $204(190-220)$ respectively and difference was statistically significant $(P<0.01)$. The myocardial performance index (MPI) was also statistically different in controls and cirrhotics $(P<0.001)$. Other parameters E: A ratio, Iso volumetric relaxation time (IVRT), interventricular septal thickness, posterior wall thickness, ejection fraction, Q-Tc was not different among the two groups. There was no difference between alcoholic and non alcoholic groups. The changes in cardiac parameters and serum BNP levels did not correlate with severity of liver disease though higher levels of serum BNP were seen in advanced liver disease. 
Conclusion: In this study, $64.2 \%$ cirrhotics had diastolic dysfunction, none had systolic dysfunction. There was no correlation between cardiac parameters and the severity of liver disease. These cardiac changes were due to cirrhosis rather than other causes.

\section{L23}

\section{Role of HSP70 protein in acute liver failure}

Ujjwal Sonika, Rajib K Hazam, Premashis Kar. Department of Medicine, Maulana Azad Medical College, New Delhi 110 002, India

Background and Aim: Heat shock proteins (Hsp) are families of phylogenetically conserved molecules that have a range of cytoprotective and intracellular functional roles. Reactivity to heat shock proteins has been implicated in the development of autoimmune disease and tissue expression of heat shock proteins and increased levels of anti-Hsp antibodies have also been reported in vascular disease. The levels of HSP70 in patients of acute liver failure (ALF) have not been studied before. This study compared circulating levels of Hsp 70 in Acute liver failure (ALF), acute viral hepatitis (AVH) cases and 30 healthy controls. Methods: 30 patient of ALF who were admitted in LNH were enrolled in study. The levels of HSP70 of ALF patients were measured on admission and compared with 30 patients of AVH and 30 healthy controls using commercially available ELISA Kit (Cusabio Biotech). The clinical characteristics and biochemical parameters were also evaluated in the studied population.

Results: The HSP70 levels were found to be significantly higher in ALF patients $(75.08 \pm 13.02 \mathrm{ng} / \mathrm{ml})(P<0.001)$ compared to AVH $(42.11 \pm 6.75 \mathrm{ng} / \mathrm{ml})$ and Healthy controls $(14.89 \pm 3.49 \mathrm{ng} / \mathrm{ml})$. ALT was significantly higher in ALF compared to AVH group and controls $(589+677 \mathrm{IU} / \mathrm{ml}$ vs $1343+1212 \mathrm{IU} / \mathrm{ml} P>0.001$ and $1343 \pm 1212 \mathrm{IU} / \mathrm{ml}$ vs. $22.6 \pm 4.9 \mathrm{IU} / \mathrm{ml} p<0.001)$ respectively. Conclusion: Higher Hsp70 levels may increase this risk of ALF and may have a predictive value in the course of the disease.

\section{L24}

\section{Clinical and laboratory profile in alcoholic cirrhosis in Sikkim}

\section{O P Dhakal, Mona Dhakal.}

Sikkim Manipal Institute of Medical Science, Gangtok, Sikkim

Methods: Retrospective analysis of computer loaded data of 48 cirrhotic patients.

Analysis: Total Cases - 48 Age (23-72 yrs) $52.8 \%$ in age group 41-50 yrs, M (47.9\%) F (52.08\%) etiology - alcohol
(81.2\%), HCV (2.08\%), others (8.33\%) Blood groups 0+ve (18.75\%), A+ve (12.5\%), B+ve (12.5\%) AB (4.16\%). Presentation ascities $(68.75 \%)$, HE $(35.41 \%)$, hemetemesis (31.25\%) jaundice (35.41\%); laboratory - $\mathrm{Hb}<12(58.8 \%)$, TLC $<4000(6.25 \%)>11000(39.4 \%)$, Platelets $<1.5(35.5 \%)$ PT diff $>4(60 \%)$, TB $>1.2(40 \%)$, albumin $<3.5(42 \%)$, ALT $>40(43 \%), \quad$ AST $>40(47 \%), \quad$ ALP $>175 \quad(43 \%)$ RBS $<70$ $(16.8 \%)>140(20.8 \%)$, urea $>40(50 \%), \mathrm{Cr}>1.4(41.60 \%)$, $\mathrm{Na}<135 \quad(10.41 \%)>155 \quad(8.2 \%), \quad \mathrm{K}<3.5 \quad(10.7 \%)>5.5$ (12.4\%), ascitic fluid TLC $>500$ (33.33\%), neutrophil predominant $(26.6 \%)$, lymphocyte predominant $(73.33 \%)$ total protein $>3.5(40 \%)$, SAAG $>1.1(86.66)$.

Conclusion: Females were more in numbers, Ascitis and HE was common initial clinical presentation. Anemia, leucocytosis and thrombocytopenia was seen at the time of presentation. Electrolyte imbalance and deranged kidney function were seen in more number of cases.

\section{L25}

Study of epidemiological profile and treatment options of hepatocellular carcinoma (HCC) patients in Indian population, single tertiary care experience

Sandeep Davavala, Rajesh Gupta, P NRao, DNageshwar Reddy. Asian institute of Gastroenterology, Somajiguda, Hyderabad 500 082, AP

Methods: Consecutive patients with cirrhosis of liver were screened for HCC by S.AFP and dynamic imaging (USG, Triphasic CT and MRI). FNAC of suspected lesion was done only if imaging and serology were not conclusive. Various treatment options feasible were analysed and treatment outcomes assessed.

Results: Seventy two cases were diagnosed as HCC over 2 years period. Mean age was 65 years affecting (M:F, 5:1), with underlying cirrhosis in $94.4 \%$. Etiology was hepatitis B (30.5\%), hepatitis C (27.7\%), NASH and cryptogenic (34.7\%), others (6\%). Mean CPT score was (A 25\%, B and C 37.5\% each) and OKUDA staging revealed (I 40.5\%, II 30.5, III 29\% patients). Morphological characteristics showed mostly single tumors, of mean size $4 \mathrm{~cm}$, in right lobe (86.6\%) and portal vein tumor thrombosis in $45.5 \%$. Ultrasonographically $50 \%$ lesions were hyperechoic and small lesions of size $<2 \mathrm{~cm}$ were uniformly hypoechoic. Triphasic CT showed heterogeneous contrast enhancement in arterial phase with washout in delayed venous phases in $91.1 \%$. FNAC was required in $44 \%$. Treatment outcomes showed curative resection (5.5\%), RFA $(13.5 \%)$, TACE $(19.4 \%)$ and conservative $(50 \%)$. Follow up $30 \%$, those receiving curative and palliative care were alive till date, $35 \%$ expired and remaining lost follow up.

Conclusion: $\mathrm{HCC}$ is a commonest complication occurring in patients with cirrhosis. Hepatitis $\mathrm{B}$ and $\mathrm{C}$ are commonest 
causes. Majority of patients are in late stages were curative treatment options are seldom feasible

\section{L26}

To evaluate efficacy of non-invasive markers of fibrosis in determining liver fibrosis vis-a-vis liver biopsy, in patients of nonalcoholic fatty liver disease.

Parveen Malhotra, Bhupinder Singh, Jasleen Kaur, Sethu Babu, Dharmesh Kapoor.

Global Hospitals, Hyderabad

Twenty-five patients of NAFLD underwent laboratory investigations including the specific non-invasive markers of fibrosis namely, haptoglobin, alpha-2 macroglobulin, apolipoprotein- A1 levels were done and liver biopsy.

Results: The waist/hip ratio had statistically significant correlation with fatty infiltration $(p=0.04$, Fisher's exact test). The HOMA- IR had statistically significant correlation with liver histology in respect to inflammation and fibrosis, even with two classifications i.e. Metavir $(p=0.03)$ and Matteoni $(p=0.02)$. The $\mathrm{P}$ value was not significant $(>0.05)$ in predicting any relation between these three and presence of fatty infiltration, inflammation, fibrosis, and Mallory's hyaline or ballooned hepatocytes. Apolipoprotein-A1 was the most common marker to be deranged in fibrosis and inflammation but it was also decreased in patients with simple steatosis. Liver biopsy revealed fibrosis in 8 patients (Metavir score, $\mathrm{F} 1=4$ and F2 $=4$ whereas Matteoni scoring was 4 in all of them). The portal and lobular inflammation was seen in $5(20 \%)$ and all of them had mild inflammation (Metavir A1 and Matteoni-2).

Conclusions: The non-invasive markers of liver fibrosis are important as they have potential to become alternative to liver biopsy but they have to be validated in larger studies.

\section{L27}

Treatment of minimal hepatic encephalopathy: a randomized control trial comparing $L$ ornithine $\mathrm{L}$ aspartate with placebo

P Puri, A Dhingra, B Nandi, A Tiwari, N Grover, S Srivastav, $S$ Shah, A C Anand.

Department of Gastroenterology, Army Hospital R \& R, New Delhi

Background: Minimal Hepatic Encephalopathy (MHE) adds to morbidity of cirrhotics by affecting cognitive skills. Aim: To evaluate role of L-Ornithine L-Aspartate (LOLA) in cirrhotics with MHE.

Methods: In this double blind randomized control trial of LOLA vs. Placebo in cirrhotics with MHE ( $\geq 2$ abnormal psychometric tests), psychometric tests [number connection tests (NCT), Digit symbol test (DST), Block design test (BDT)], neurophysiological tests [Critical flicker frequency (CFF) and P300] and ammonia levels were done at baseline and after 2 weeks of therapy.

Results: Of the 78 patients with MHE (M 67, F 11 mean age $41.12 \pm 9.428$ years), 03 were excluded in LOLA group (liver transplant-02, SBP-01) and 06 in placebo group (liver transplant -01 , infections / GI bleed 03, death 02 (road traffic accident 01 , GI bleed 01$)$. In the LOLA group $(n=36)$, all neuropsychological and neurophysiological tests showed improvement. (NCT $42.37 \pm 7.46$ vs. $37.79 \pm 6.28$; $p<0.001$; BDT $92.0 \pm 17.15$ vs. $81.81 \pm 14.46 ; p<0.001$; DST $8.00 \pm$ 2.34 vs. $9.50 \pm 1.87$; p 0.001 ; CFF $35.19 \pm 3.48$ vs. $37.03 \pm$ 3.53 ; p 0.007 ; P300 $380.06 \pm 31.70$ vs.362.28 $\pm 34.61 ; \mathrm{p}$ $0.029)$. In the Placebo group $(n=33)$, only DST and BDT showed improvement. (NCT $40.63 \pm 7.81$ vs. $39.37 \pm 7.46$; $p$ 0.205 ; BDT $91.09 \pm 13.73$ vs. $82.52 \pm 12.95$; $p<.001$; DST $7.55 \pm 2.41$ vs. $9.03 \pm 1.75$; p 0.001 ; CFF $35.29 \pm 3.77$ vs $35.136 \pm 2.82$; p 0.833 ; P300 $384.03 \pm 29.152$ vs. $369.30 \pm$ 33.71 vs; $p$ 0.069) The ammonia levels were lower in both arms (LOLA $96.83 \pm 11.77$ vs $85.15 \pm 18.19$; p 0.008 , Placebo $95.89 \pm 11.39$ vs. $83.80 \pm 16.98$; p 0.002 ).

Conclusion: LOLA has been shown to be beneficial in management of MHE.

\section{$\mathbf{L 2 8}$}

A prospective study on incidence, etiology, risk factors and outcomes of hepatic dysfunction after hematopoietic stem cell transplantation

S K Rastogi, A K Seth, P Puri, B Nandi, V Nair. Departments of Gastroenterology and Hematology, Army Hospital (Research \& Referral), New Delhi.

Background and Aims: To study the incidence, etiology, risk factors and outcomes of hepatic dysfunction after autologous and allogeneic hematopoietic stem cell transplantation (HSCT). Methods: Consecutive patients undergoing HSCT from Jan07 to Dec08 were studied prospectively. Pre-transplant evaluation included LFT, HBsAg, total antiHBc, antiHCV, HBVDNA and HCV RNA of patient and donor. Note was made of conditioning regimen and immunosuppression. Post transplant LFT was done daily for 30 days and then weekly for 3 months. Viral markers and imaging were done as relevant. Results: 57 HSCTs, 36 (63.2\%) allogeneic and 21 (36.8\%) autologous were done in 55 patients. Indication was AML 19, Myeloma 12, ALL 7, Thalasemia 6, Aplastic anaemia 6 and miscellaneous 7. Pre-existent liver dysfunction was present in 7 (HBV 2, leukemic infiltration 1, ATT induced 1 , iron overload 1 , indeterminate 2). Median follow up was 9 (range 0-27) months. Liver dysfunction developed in 22/ $36(61.1 \%)$ of allogeneic (Sinusoidal Obstruction Syn- 
drome 10, GVHD 3, drug induced 2, CMV 2, sepsis 2, iron overload 1 , disease recurrence 1 and indeterminate 1) and 6/21 (28.6\%) autologous (drug induced 5, sepsis 1) HSCTs. Mortality occurred in 16 of 36 (44.4\%)allogeneic and 6 of 21 (28.5\%)autologous recipients. Liver dysfunction was the cause of death in 5 of $16(31.2 \%)$ allogeneic but none of the autologous HSCTs. None of the patients transplanted with pre-existent liver dysfunction survived $(p=0.01)$.

Conclusions: Liver dysfunction is more common after allogeneic as compared to autologous HSCT. The most common cause is sinusoidal obstruction syndrome following allogeneic and drug hepatotoxicity after autologous transplantation. Pre-existent LFT abnormality is associated with high mortality.

\section{L29}

\section{Study of transaminase levels in South Malabar population}

Vinaya Raghavendra, ${ }^{\#}$ Mahesh Pandian, ${ }^{\#}$ Santhosh, ${ }^{*}$ Saju Xavier.*

Departments of ${ }^{\#}$ Internal Medicine, and *Gastroenterology, Al Shifa Hospital, Perintalmanna, Kerala

Introduction: Transaminase levels form the basis on which therapeutic decisions in chronic hepatitis are made. Twice the the upper limit of normal is usually taken as the threshold for treatment. Recent studies have suggested Revision of the normal upper levels of ALT.

Aims: To study the AST and ALT levels in an apparently healthy population. To define ALT and AST levels in a population without any risk factor for liver disease. To compare the ALT and AST levels in patients with and without metabolic risk factors.

Methods: Retrospective study from a hospital database of executive health check up. Patients with overt liver disease and high risk behaviour of liver disease and those with altered lipid levels, blood sugars obesity and sonologic fatty liver were excluded to form a subgroup of healthy population and transaminase levels estimated using standard procedures. Patients with metabolic syndrome were also analysed separately. Results: A total of one thousand case files were analysed and after exclusion of all risk factors detailed above, there were 130 healthy population and the $95 \%$ confidence intervals for ALT values were 31.29 and 42.86 for males and 18.26 and 22.17 for females. In the metabolic group the ALT levels were 46.03 and 64.45 in males and 22.07 and 32.63 In females.

Conclusion: The ALT levels in this population is higher than the levels recommended in the west. The patients with metabolic syndrome have significantly higher ALT levels. The values in females were significantly lower than in males.

\section{L30}

Pregnancy outcome is poor in women with hepatic venous outflow tract obstruction (HVOTO)

Anupam Mahapatra, Akash Shukla, Anumeet Singh Grover, Shobna Bhatia.

Department of Gastroenterology, Seth GS Medical College and KEM Hospital, Parel, Mumbai 400 012, India

Introduction: There are limited data of effect of HVOTO on fertility and none on fertility or pregnancy profile prior to symptom-onset in HVOTO. We evaluated the fertility and outcome of pregnancy in patients with HVOTO.

Methods: Forty-one consecutive married women (age $30.8 \pm$ 5.8 years), who were sexually active for at least 2 years and not using contraception, were prospectively included. Their detailed obstetric history was obtained.

Results: The obstruction was at hepatic vein (HV) in 25 , inferior vena cava (IVC) in $6 ; 11$ patients had IVC+HV thrombosis. The median duration of symptoms was 3 months [0.5-48]. Prothrombotic states were identified in 6 (APLA-3, FactorVLeiden-1, PNH-1, FVL+APLA-1). Six patients had primary infertility; 35 women conceived 85 times. 22 had 42 live births; 19 had 28 first trimester abortions, 4 had 7 second trimester abortions and 4 had one still birth each; medical termination of pregnancy was done in 4 patients. More patients with second trimester abortion had prothrombotic state than those without any abortions ( $3 / 4$ vs $2 / 14, p=0.04$, $\mathrm{OR}=18.0$ [1.2-271.5]). Prothrombotic states did not correlate with infertility. Women who had live births were older at diagnosis $(33.1 \pm 6.1$ vs $28.2 \pm 3.8$ years; $p=0.008$, OR 5.5 [1.6-9.4]) and at onset of symptoms (32.4 \pm 6.2 vs $27.0 \pm$ 3.9 years; $p=0.023$, OR $4.5[1.2-17.1]$ ), as compared to those who never had any live births. Pregnancy outcome and infertility did not correlate with anatomy of $\mathrm{HV}$ or IVC obstruction and duration of symptoms.

Conclusion: Women with HVOTO have poor outcome of pregnancy even before the onset of the liver-related symptoms.

\section{Gall Bladder}

\section{GB1}

\section{ERCP in geriatric population-an audit}

D Sasi Anand, A Chezhian, K Muthu Kumaran, S Chitra, $G$ Ram Kumar, $R$ Balamurali, $T$ Pugazhendhi, S Jeevan Kumar.

Department of Digestive Health and Diseases, Government Peripheral Hospital (Kilpauk Medical College), Anna Nagar, Chennai 600 102, India 
Aim: To present the indications, safety and efficacy of ERCP in the Geriatric population.

Methods: This is a retrospective study evaluating the indications, safety and efficacy of ERCP in Geriatric patients admitted to our department. All patients underwent appropriate clinical, biochemical and radiological investigations. All patients underwent ERCP during the period January 2008 to July 2009. Indications for ERCP include CBD stone disease, CBD stricture, CBD stone with stricture and palliation for periampullary growth.

Observation: 43 patients (M:F-18:25) aged between 65 and 78 years (Mean 69.63 yrs) were included for this study. ERCP was successful in 36 cases $(83.72 \%)$ and failed in 7 cases (16.28\%). CBD stones were reported in 24 cases (55.82\%), CBD stricture in 4 cases $(9.30 \%)$, CBD stricture with stones in 5 cases $(11.63 \%)$, and periampullary growth in 10 cases (23.25\%). Associated findings were periampullary diverticulum in 12 cases (27.90\%), post cholecystectomy state in 3 cases (6.98\%). Sphincterotomy was done in 28 cases $(65.12 \%)$, basketing in 2 cases (4.65\%), balloon trolleying in 15 cases $(30.23 \%)$, biliary dilatation in 1 case $(2.32 \%)$ and biliary stenting in 34 cases $(79.06 \%)$. Relief of cholangitis and jaundice were seen in all cases. Post procedure mild pancreatitis and fever were noted in 4 cases each $(9.30 \%)$. No procedure related mortality was noted.

Conclusion: ERCP is a safe and effective procedure in the Geriatric population in experienced hands. CBD stone disease remains the most common indication for ERCP in this population. In this study, there are no major procedure related complications noted.

\section{GB2}

\section{Chromosomal structural analysis in carcinoma of the gall bladder}

Prashant Chandra Das, Prabhat Kumar, Rajnikant Mishra*, Somprakas Basu, Vijay K Shukla.

Department of General Surgery, Institute of Medical Sciences, Department of Zoology,* Banaras Hindu University, Varanasi 221005 , India

Objective: Gall bladder cancer is the most lethal malignancy of the biliary tract with largely unknown etiology and poor survival. Mounting evidence suggest that cancer is a genetic disease and most neoplasms are in origin. Acquire or inherent genetic instability in normal cells causes mutational events that results in neoplastic transformation. This study was aimed to analyse chromosomal abnormalities in carcinoma of the gall bladder patients.

Method: Thirty cases of histologically proven carcinoma of the gallbladder cancer screened for there chromosomal abnormalities in there blood lymphocyte. Metaphase plate was prepared through lymhocyte culture followed by Gbanding. Chromosomal abnormalities was analysed after karyotyping. Enzyme activity assay for LDH, catalase and SOD activities was carried out using polyacrylamide gel (8\%) electrophoresis.

Results: Four patients with simple chromosome breakage and one translocation was observed. All aberrations are present in males. The serum shows high activity of LDH2 in carcinoma gallbladder with elevated activity of catalase and Mn-SOD.

Conclusion: The simple aberrations in form of breakage and translocations are suggestive of underlying genetic predisposition for carcinoma of the gallbladder. These aberration can act as a marker chromosome for carcinoma of the gallbladder.

\section{GB3}

Role of dexamethasone in prevention of post operative nausea and vomiting after laparoscopic cholecystectomy

Prashant Chandra Das, Somprakas Basu, Raman Singh, Vijay Kumar Shukla.

Department of General Surgery, Institute of Medical Sciences, Department of Zoology, ${ }^{*}$ Banaras Hindu University, Varanasi 221005 , India

Objective: Laparoscopic cholecystectomy (LC) is presently the gold standard for treatment of gallstone disease (GSD). Its benefits include a shorter hospital stay, lesser postoperative pain and better cosmesis. However a high incidence of post operative nausea and vomiting (PONV) is also a side effect. Various drugs have been used to ameliorate PONV with mixed results. We intend to evaluate the effect of dexamethasone on PONV following LC.

Method: A randomised controlled study was done over a period of six months in which 66 cases of GSD were taken and divided into 33 test and 33 control each randomly. Demographic and operative data were collected. Preoperative Inj. dexamethasone $(8 \mathrm{mg})$ was given intravenously at the time of induction of anaesthesia. Pain and PONV were assessed at 12 and $23 \mathrm{hrs}$ postoperatively. Student's $t$ test, Mann-Whitney U test, Chi square test, and Fischer's exact test were used as required for test of significance.

Result: The patients age, BMI, disease duration, operative time, carbon dioxide used and type of stones were comparable in both the groups. Out of 33,4 patients $(12.1 \%)$ of the test group had nausea as compared to 10 out of $33(30.3 \%)$ in control group in the first $12 \mathrm{hrs}$ ( $p$-value $=$ 0.02 ). However no significant difference in nausea at $23 \mathrm{hrs}$ and vomiting or pain at 12 and $23 \mathrm{hrs}$ was observed between the groups. 
Conclusion: Preoperative dexamethasone significantly decrease postoperative nausea following LC.

\section{GB4}

\section{A study of ERCP abnormalities in cases of post cholecystectomy biliary leaks}

Nalini Prasad Ippela, Ratnagiri, Sunil Kumar, Ramanna, M Uma Devi, A Vidyasagar.

Department of Gastroenterology, Gandhi Medical College and Hospital, Secunderabad. AP, India

Background: Significant bile leak is an uncommon complication after biliary surgery. Its incidence had been rising In Laparoscopic era. ERCP imaging in these cases helps define the site of leak and severity of injury and hence plan subsequent management.

Method: 33 patients presented with bile leaks after cholecystectomy (Open cholecystectomy in 9 cases, laparoscopic cholecystectomy in 20 cases, Open cholecystectomy and CBD exploration in 4 cases) during the period 2005-2010.Preoperative details regarding LFT, USG abdomen and indication for surgery were reviewed in all these patients. ERCP was done after patient condition was stable and fit for procedure. The site of leak was demonstrated on ERCP. Biliary endotherapy as feasible was done.

Results: Bile leaks in 33 patients occurred from cystic duct in 16 patients (48\%), CBD in 10 cases (30\%),right hepatic duct in 3 cases $(9 \%)$, intrahepatic ducts in 4 cases (12\%). complete $\mathrm{CBD}$ transection was noted in 3 cases. Bilary endotherapy in form of sphincterotomy and biliary stenting was possible in 27 patients ( $82 \%)$. In one case balloon sphincteroplasty followed by stone removal was done. Bile leaks stopped in treated cases at a mean interval of 4 days. No major complication was noted after the procedure. Surgery was done in the remaining cases with uneventful recovery.

\section{GB5}

\section{Associative role of MTHFR gene mutation and altered homocysteine levels in gallbladder disease severity}

Bharati Baruah, ${ }^{1}$ Diptika Tiwari, ${ }^{1}$ Md. Ghaznavi, ${ }^{1}$ Moumita Bose, ${ }^{1}$ Sujoy Bose, ${ }^{1}$ Anjan Saikia, ${ }^{2}$ Rajkumari Deblakshmi, ${ }^{1}$ Manashree Sharma, ${ }^{1}$ Manab Deka. ${ }^{\text {. }}$

${ }^{1}$ Deparment of Biotechnology, Gauhati University, Guwahati, Assam, ${ }^{2}$ Central Hospital, N.F. Railway, Maligaon, Guwahati, Assam, India

Background: Underlying molecular etiology of gallbladdercarcinoma (CaGB) development is not well elucidated.
Aim: Evaluate genetic alteration in MTHFR gene involved in folate metabolism and DNA repair, altered plasma homocysteine levels, in predisposing patients to gallbladder anomalies.

Methods: Gallbladder disease patients \{cholelithiasis $(n=$ $20)$, cholecystitis $(n=20)$ which are risk factors for $\mathrm{CaGB}$, and $\mathrm{CaGB}(n=8)\}$ admitted to Central Railway Hospital, Guwahati, and age and sex matched community controls $(n=$ 20 ) were enrolled for the study. Genomic DNA was extracted from blood, and MTHFR677Cï $f$ genotyping was performed by PCR-RFLP. Plasma homocysteine levels were determined commercially. Statistical analysis was performed using SPSSv13 software.

Results: Prevalence of MTHFR677Cif T mutant genotypes was higher in $\mathrm{CaGB}(87.5 \%)$ compared to cholelithiasis (35\%), cholecystitis (55\%) and controls (25\%). Overall, presence of mutant genotype non-significantly increased the risk of gallbladder diseases. Importantly, presence of mutant genotype increased CaGB risk in cholelithiasis patients significantly $\{\mathrm{OR}=13.0 \quad(1.319-128.106), p=$ $0.033\}$ and cholecystitis non-significantly $\{\mathrm{OR}=5.727$ (0.590-55.6), $p=0.199\}$. Wilcoxon Signed Ranks test of correlation showed that homocysteine levels was significantly higher in the mutated cases $(p=0.012)$. Level of homocysteine was elevated for homozygous $(p=0.026)$ and heterozygous mutant $(p<0.001)$ cases compared to wild type. Interestingly, the homocysteine levels were higher for wild type CaGB cases compared to other groups with wild type MTHFR genotype.

Conclusion: Mutation in MTHFR gene and its influence on homocysteine levels may predispose patients to gallbladder anomalies and even play a role in disease progression and severity.

\section{GB6}

Role of Wnt-b-catenin pathway deregulation in gall bladder disease severity

Diptika Tiwari, ${ }^{1}$ Rajkumari Deblakshmi, ${ }^{1}$ Manab Deka, ${ }^{1}$ Moumita Bose, ${ }^{1}$ Bharati Baruah, ${ }^{1}$ Anjan Saikia, ${ }^{2}$ Sujoy Bose. ${ }^{l}$ ${ }^{1}$ Deparment of Biotechnology, Gauhati University, Assam, ${ }^{2}$ Central Hospital, N.F. Railway, Maligaon, Guwahati, Assam, India

Background: Gallbladder anomalies including carcinoma gallbladder $(\mathrm{CaGB})$ cases are alarmingly increasing in $\mathrm{NE}$ India. Alteration and deregulation of Wnt-b-catenin pathway is implicated in tumor progression and carcinogenesis of multiple etiology.

Aim: Screening of genetic and transcriptional alterations of b-catenin in gall bladder disease patients from Northeast India. 
Methods: Mutation analysis of b-catenin exon3 region was done by extracting DNA from whole blood of 21 gall bladder disease patient \{cholelithiasis $(n=11)$, Cholecystitis $(n=7)$ and CaGB $(n=3)\}$, enrolled from Central Railway Hospital, Guwahati followed by PCR-RFLP analysis. Total RNA was isolated by Trizol method and converted to cDNA. Deregulation of expression of b-catenin was studied for all chronological stage of gallbladder diseases and healthy controls by semi quantitative RT- PCR at m-RNA level using $\hat{I}^{2}$-actin as internal control.

Results: Amongst the 21 cases enrolled, b-catenin mutation was found in 1 cholelithiasis case only. The difference in the distribution of mutation was not significant when compared between cholelithiasis and cholecystitis $(p=0.779)$ or between cholelithiasis and GBC $(p=0.875)$ cases. Meanwhile b-catenin expression was upregulated chronologically in the gallbladder diseases, least being in healthy control and highest in CaGB cases, indicating deregulation of the Wnt-b-catenin pathway irrespective of the b-catenin mutation status.

Conclusion: Alteration in expression profile of b-catenin is associated with progression and severity of gallbladder anomalies. Further in-depth experimental work on Wnt-bcatenin at large scale is required to draw conclusive outputs.

\section{GB7}

\section{Clinical and radiological findings in adult patients with choledochal cyst (CDC) in tertiary care centre}

\author{
A K Rawat, ${ }^{1}$ A S Puri, ${ }^{1}$ S Sachdeva, ${ }^{1}$ S K Puri. ${ }^{2}$ \\ ${ }^{1}$ Department of Gastroenterology, ${ }^{2}$ Department of Radiology, \\ G B Pant Hospital, New Delhi 110 002, India
}

Methods: Adult patients diagnosed to have $\mathrm{CDC}$ on the basis of USG, MRI/MRCP, and ERCP were taken as the index patients. First degree relatives of the index patients were subsequently screened for hepatobiliary-pancreatic abnormalities by liver function test (LFT), USG abdomen.In those where either LFT or USG were abnormal, were subjected to further testing by CT, MRI, or ERCP. Detailed analysis were made of the clinical presentation, biochemical and radiological findings. Results: Over a 2 year period 92 patients were diagnosed to have CDC (age $46.6 \pm 15.5$ years). Females constituted $78 \%$ $(n=72)$ of the study population. Abdominal pain was the presenting feature in $91(98.9 \%)$ of the 92 patients. Mean duration of abdominal pain before diagnosis was 20 months (range 1-240 months). Other presenting features were jaundice in 12 (13\%); fever in 4 (4.3\%); 11 patients $(12 \%)$ had undergone previous cholecystectomy for the abdominal pain. Subtypes of CDC seen: type1-74 (80.4\%); type2-2 (2.2\%); type 4A-15 (16.3\%); 4B-1 (1.1\%). Cystolithiasis was present in $45(48.9 \%)$ as documented on MRCP/ERCP. Gallstones seen in 49 (53\%). Intrahepatic stones in 1 (1.1\%).
Mean bilirubin value $1.8 \mathrm{mg} \%$ (0-25 mg\%),SAP $300 \pm$ 189 U/L (23-1769 U/L).Four (4.3\%) patients were diagnosed to have cholangiocarcinoma.None of the patients had APBDJ. Of 713 first degree relatives 125 (17.5\%) were screened. 3 first degree relatives (3.3\%), (all female offsprings of index cases) were also found to have CDC type1.

Conclusion: CDC are common in adult patients. Commonest presenting manifestations are abdominal pain and jaundice. Commonest complication seen in adult patients are cystolithiasis and cholangiocarcinoma.

\section{GB8}

To compare the diagnostic yield of fine needle aspiration cytology, surface ampullary biopsy and needle knife papillotomy assisted ampullary biopsy in periampullary tumors

Ram Chandra Soni, Sanjeev Sachdeva, Pankaj Tyagi, $R$ K Saran,* A S Puri.

Department of Gastroenterology and *Pathology, G B Pant Hospital, New Delhi 110 002, India

Preoperative diagnosis needs to be established in periampullary tumors (PAT) as survival is better in them vis-a-vis other bilio-pancreatic malignancies. Current practice to take biopsies from papilla has poor histopathological yield when there is no ulceration. We hypothesized that tumors deep in submucosa of ampulla would have poor yield with FNAC or surface biopsies. If NKP assisted biopsy (NKAB) can be done by cutting ampullary bulge and taking deep biopsies, it can improve histopathological yield.

Aim: To compare diagnostic yield of FNAC, Surface biopsy and NKAB in PAT with smooth mucosa. Material methods: Patients with PAT were included. Patients with G.O.O, ampullary ulceration were excluded.

Results: 78 patients were included of which 27 (34\%) had smooth ampullary bulge. $48 \%$ were male. Median age was 60 yrs. Clinical parameters showed jaundice (70\%), pain (76\%), mass (64\%), fever (40\%), weight loss (88\%). Mean CA19.9 level was $466 \mathrm{U} / \mathrm{ml}$. Surface biopsy was positive in 10/25 (PPV$40 \%$ ), FNAC in 12/25 (PPV-48\%) and NKAB in 21/25 (PPV$84 \%)$ for adenocarcinoma. There was no significant difference in between FNAC and surface biopsy $(P=0.56)$. There was significant difference in between FNAC and NKAB $(P<$ $0.007)$ and surface biopsy and NKAB $(P<0.001) .10$ patients, who were NKAB positive, underwent Whipples and resected specimen confirmed malignancy. Of the 21 patients positive for adenocarcinoma, well, moderately and poorly differentiated adenocarcinoma was found in 12,7 and 2 patients respectively. 2 (7\%) patients had neuroendocrine tumor.

Conclusions: NKAB is better than FNAC and surface biopsy. 


\section{GB9}

Comparing the efficacy of $7 \mathrm{~F}$ vs $10 \mathrm{~F}$ plastic stents in malignant biliary obstruction-a tertiary care review

S Babu kumar S, T Arun, K Ram Kumar, K Muthu Kumaran, $R$ Bala Murali, S Jeevan Kumar, T Pugazhendhi.

Department of Digestive Health and Diseases, Government Peripheral Hospital, Anna Nagar, Chennai 600102 and Kilpauk Medical College, Chennai 600 010, India

Aim: To compare the effectiveness of $7 \mathrm{~F}$ and $10 \mathrm{~F}$ in endoscopic palliation of jaundice.

Materials: 60 patients, males (36), females (24), ranging 32-80 years form the basis of the study conducted from December 2007 to December 2009.

Methods: LFT, USG and MRCP (in selected cases) were done in all patients in this study group. Patients were divided into two groups: those with $7 \mathrm{Fr}$ and $10 \mathrm{Fr}$ stent. Unilateral random biliary stenting was done. Patients were observed for 2 days. After 1 week patients were assessed for improvement. Patients are said to have adequate drainage if S. Bilirubin falls by at least $50 \%$ by 7 days.

Results: The overall success rate for endoscopic stenting was $68.33 \%$; Success rate for proximal and $60 \%$ distal Biliary Obstruction was $60 \%$ and $78 \%$ respectively. Number of cases with $10 \mathrm{Fr}$ stent-16.Number of cases with 7Fr stent -25 .Mean number of days of stent patency after $10 \mathrm{~F}$ stent was 217 days and 61 days after $7 \mathrm{~F}$. Most common complication was cholangitis (3), pancreatitis (1), perforation (1), migration (1). Complication rate following $7 \mathrm{~F}$ stenting was 3 patients compared to 1 patient following $10 \mathrm{~F}$. Mean Serum Bilirubin (total) before stenting was 14.44 and after stenting was 7.19. Repeat T.Bilirubin 7 days following $7 \mathrm{~F}$ and $10 \mathrm{~F}$ stenting were 5.3 and 7.1.

Conclusion: Both $7 \mathrm{~F}$ and $10 \mathrm{~F}$ stents are equally effective in reducing jaundice where as $10 \mathrm{~F}$ stent has high patency rate. Complication rate were similar in both groups.

\section{GB10}

\section{Redefining the role of liver function test in cholelithiasis}

Vivek Srivastava, Vaibhav Pandey, Mumtaz A Ansari, Anand Kumar.

Background: Cholelithiasis without obstructive jaundice is investigated with Liver Function Tests (LFT) invariably. Deranged LFT is a common indication for ERCP, MRCP or doing open cholecystectomy.

Objective: To evaluate the usefulness of routine LFT in patients with symptomatic cholelithiasis.
Methods: A retrospective study was done on symptomatic cholelithiasis admitted between May 2008 to July 2010. There was no past or present history of jaundice, pancreatitis or cholangitis. Common bile duct diameter was normal on ultrasonography. The data of LFT, CBD diameter and type of operation was analyzed.

Results: A total of 104 patients $(89 \mathrm{~F}: 15 \mathrm{M})$ were included. The mean age was $42 \pm 14.6$ years. Chronic cholecystitis was present in $95(91.3 \%)$ patients and acute cholecystitis in 9 (8.7\%) patients. In 78 patients (Group A) LFT was normal and in 26 (Group B) it was abnormal. In Group A 75 (96\%) patients had chronic cholecystitis while 3 (4\%) had acute cholecystitis. In Group B, 20 (77\%) had chronic cholecystitis while $6(23 \%)$ had acute cholecystitis. Bilirubin was found elevated in 16, AST in 22 and alkaline phosphatase in 20 patients. Patients having deranged alkaline phosphatase underwent open cholecystectomy while in others laparoscopic cholecystectomy ( \pm conversion) was done. None of the patients undergoing open procedure for deranged LFT had CBD dilatation or palpable stones during operation. No patient developed CBD stone during follow up.

Conclusion: A routine LFT in cholelithiasis with normal CBD diameter on ultrasound usually comes normal and even if deranged, it is not a predictor of CBD calculus.

\section{GB11}

\section{One year audit of ERCP - a retrospective study}

A Chezhian, D Sasianand, G Ramkumar, K Muthukumaran, $S$ Chitra, R Balamurali, T Pughazhendhi, S Jeevankumar. DDHD, Government Peripheral Hospital, Anna Nagar. Kilpauk Medical College Chennai

Aim: To analyse the indication, outcome and complications of ERCP done in a tertiary care hospital.

Methods: A retrospective analysis of ERCP done between july2009 to June 2010. All the records were maintained at our hospital.

Results: A total of 359 patients underwent ERCP during that period. Out of which 350 Cases were therapeutic and 9 cases were diagnostic. Youngest patient is 9 years old and oldest patient is 82 years old. Most common indication is choledochothiasis in 140 cases $(40 \%)$ followed by periampullary growth in 100 cases $(29 \%)$, Biliary stricture in 40 cases $(11 \%)$, Bile duct injury 10 cases $(3 \%)$,hilar cholangiocarcinoma 10 cases (3\%), acute pancreatitis in 20 cases $(6 \%)$,chronic pancreatitis in 18 cases $(6 \%)$. Some rare indications are hepatolithiasis, pancreas divisum, choledochal cysts, hydatid cyst, Mirizzi syndrome and upmigrated stent. Success rate is $99 \%$ in case of cholidocholithasis and nearly $95 \%$ in other indications. Main reason for failure is distorted anatomy. Complications were developed in 9 
cases $(2.5 \%)$ only. 4 cases of pancreatitis, 2 retroduodenal perforations and 3 cases of cholangitis. All of them were treated medically and improved. No death was reported.

Conclusions: ERCP is a very good therapeutic tool complications are very rare.cholidocholithiasis is the most common indication.

\section{GB12}

A study to assess the safety and efficacy of EUS vs. ERCP for pts with suspected choledocholithiasis

S T Rajanesh Reddy, K Panduranga Rao, K Venugopal Reddy, $G$ Mohan Reddy, K Sathyanarayana, P Anitha.

Department of Gastroenterology, Osmania Medical College, Hyderabad, India

Background: ERCP is considered gold standard for diagnosis and treatment of choledocholithiasis but is associatied with morbidity like post ERCP pancreatitis. EUS is an alternate means of diagnosing choledocholithiasis and reduces need for diagnostic ERCP.

Objective: To study the safety and efficacy of EUS vs ERCP in management of choledocholithiasis.

Methods: 25 pts with clinical, biochemical and radiological suspicion of biliary obstruction are included in the study over a period of one year. Interventions done are EUS, ERC, sphincterotomy, and balloon sweeping of CBD when needed. Results: 10 out of 25 pts are diagnosed to have choldocholithiasis by EUS (40\%). Subsequent ERCP performed in 14 pts $(56 \%)$. Indications for ERCP are CBDstones $(n=10)$ and abnormal liver function tests $(n=4)$. Choledocholithiasis indentified in 8 of 14 pts. In 2 pts choledocholithiasis identified by EUS but not by ERCP. In ERCP group 2 pts has pancreatitis and 3 pts has bleeding. No complications observed in EUS group. With initial screening by EUS, ERCP can be avoided in $44 \%$ of pts and its complications. Conclusion: In pts with suspected choledocholithiasis EUS is safe and effective test compared with ERCP. It can be used as intial screening modality in pts with suspected choledocholithiasis and allows more selective use of ERCP.

\section{GB13}

Role of endoscopic ultrasound (EUS) in evaluation of unexplained common bile duct dilatation on MRCP

S S Rana, D K Bhasin, K Singh.

Department of Gastroenterology, Post Graduate Institute of Medical Education and Research, Sector 12, Chandigarh 160 012, India
Aims: Dilated common bile duct (CBD) without obvious cause is a not an uncommon finding on MRCP. This study was done to evaluate the diagnostic performance of EUS in patients with unexplained dilated CBD on MRCP.

Methods: Retrospective analysis of patients referred for EUS evaluation of a dilated CBD was done. The liver function tests (LFT) prior to EUS and subsequent outcome after EUS was analysed. The patients were also contacted to confirm the final outcome.

Results: Over a twenty month period, 37 patients with dilated CBD were referred for EUS. Nine patients had abnormal LFT and 28 patients had normal LFT. The EUS findings in 9 patients with abnormal LFT were: CBD stone (6), cholangiocarcinoma (2) and benign CBD stricture (1). These findings were confirmed on surgery or ERCP performed subsequently. The EUS findings in 28 patients with normal LFT were: CBD stone (9), and benign CBD stricture (1) and normal findings (20). These findings of CBD stone or stricture was confirmed on subsequently performed ERCP. Out of 20 patients with normal CBD on EUS, 14 patients were post cholecystectomy. These patients with normal biliary tree on EUS subsequently remained well without requiring further investigations (follow-up: 2 20 months). There was no difference in the mean CBD diameter between the group with demonstrable pathology compared with those without.

Conclusion: EUS is an useful investigational modality for patients with unexplained dilated CBD. The mean CBD diameter and the presence of normal liver function tests were not predictive of underlying pathology.

\section{GB14}

Differentiation between malignant and benign obstructive jaundice: does lipid profile make the task simpler?

Gaurav Gupta, S Nijhawan, A Sharma, B Sapra, A Mathur, S Nepalia.

Department of gastroenterology, S M S Medical College, Jaipur, Rajasthan, India

Objectives: The aim of this study was to investigate the effect of biliary obstruction on changes in serum lipid profile in benign and malignant conditions and to ascertain their utility as biochemical markers in differentiating them. Methods: Sixty one patients (31 malignant, 30 benign) who underwent imaging, endoscopic retrograde cholangiopancreatography, FNAC/histology with the clinical picture of obstructive jaundice were included from February 2010 to July 2010. Results: In the malignant group, significantly higher cholesterol $(p<0.001)$, low-density lipoprotein $(p<0.001)$, and triglycerides $(P<0.001)$ were observed, whereas high- 
density lipoprotein $(p<0.001)$ levels were lower. Statistical analysis showed that low-density lipoprotein $\geq 146.5 \mathrm{mg} / \mathrm{dl}$ (sensitivity $71 \%$ specificity $100 \%$ ) and high-density lipoprotein $\leq 20.5$ (sensitivity $100 \%$ specificity $97 \%$ ) were strong predictors of malignant biliary obstruction.

Conclusion: Serum lipid profile may be used as an adjunctive marker to identify malignant causes of the obstructive jaundice.

\section{Pancreas}

\section{P1}

Systemic inflammatory response syndrome (SIRS) score at admission predicts the development of primary intra-abdominal infection in patients with acute pancreatitis

Rupjyoti Talukdar, ${ }^{*}$ Santhi Swaroop Vege, ${ }^{\#}$ Magdalen Clemens. ${ }^{\#}$ *Division of Gastroenterology, NEMCARE Hospital, Guwahati, Assam, India; "Miles and Shirley Fiterman Center for Digestive Diseases, Mayo Clinic College of Medicine, Rochester, MN, USA

Background and Aim: Presence of organ failure in acute pancreatitis (AP) is associated with infected necrosis and high mortality. There are no known early predictors of intraabdominal infection (IAI) in AP. Aim of this study was to assess the capability of simple baseline parameters to predict IAI.

Methods: We studied 274 consecutive patients with AP admitted to Mayo Clinic hospitals over a 2-year period. We identified patients with microbiologically confirmed infections of pancreatic necrosis and peripancreatic fluid collections; and defined primary IAI as infection that developed prior to any abdominal intervention. We recorded admission hematocrit, BMI, BUN/creatinine and SIRS score. We used univariate and multivariate conditional logistic regression for statistical analysis and expressed the results as odds ratio (OR) [95\% confidence intervals $(\mathrm{CI})]$.

Results: 44 (16.1\%) patients had infections. 26 (9.5\%) had IAI and $20(79.9 \%)$ had primary IAI. Among these patients, $8(40 \%)$ had gram positive, $1(5 \%)$ gram negative and 11 (55\%) mixed bacterial infection. Five (25\%) had additional fungal infection. On univariate analysis, BUN $>25 \mathrm{mg} / \mathrm{dL}$ and SIRS $>2$ were found to significantly predict primary IAI with OR $(95 \% \mathrm{CI})$ of $2.68(0.96-6.96)$ and $3.59(1.42-$ 9.51) [2-tailed $p=0.048$ and 0.007 respectively]. On multivariate analysis, only SIRS $>2$ was found to be significantly predictive with an adjusted OR $(95 \% \mathrm{CI})$ of $3.13(1.21-8.49)[p=0.018]$. The other study parameters did not predict the development of IAI.
Conclusions: SIRS is a simple severity assessment tool that can predict development of primary IAI in AP early in the disease course.

\section{P2}

Role of serum amylase and lipase in predicting post ERCP pancreatitis

Mohd Abdullah Wani.

Batra Hospital and Medical research centre, New Delhi

Aim: To ascertain predictive value of serum amylase and lipase for post ERCP pancreatitis.

Methods: Between July 2009 and June2010, 51 patients under going ERCP were studied. Serum amylase, lipase concentrations were measured before the procedure and 2, 6 , and $24 \mathrm{hrs}$ later. The relationship of pancreatitis to the enzyme levels was evaluated.

Results: Post ERCP pancreatitis occurred in 4 patients (7.8\%), 75\% had moderate, $25 \%$ mild and none developed severe pancreatitis. In univariate analysis serum amylase and lipase values at 2, 6,24 hrs, and pulse rate at $2 \mathrm{hrs}$ post ERCP were found to be significant, while only post ERCP $2 \mathrm{hrs}$ serum amylase and tachycardia were significant in multivariate analysis. ROC curve of both, the $2 \mathrm{hr}$ serum amylase level and pulse rate after ERCP showed good test performance, with area under the curve of 0.98 (95\% CI: $0.95-\mathrm{I})$ and 0.95 (95\% CI:0.89-1) respectively. A total of $75 \%$ of patients with post ERCP pancreatitis had sustained rise of both amylase and lipase. There was no significant increase in risk of post ERCP pancreatitis among obese, younger patients, and women.

Conclusion: Transient rise of pancreatic enzymes post ERCP is common, majority of patients with post ERCP pancreatitis have sustained rise. $2 \mathrm{hr}$ post ERCP amylase and tachycardia are the predictors of pancreatitis.

\section{P3}

\section{A case of complicated chronic pancreatitis}

Kani Shaikh Mohammed, B Vinoth, Biggs Saravanan, M Malarvizhi, K Premkumar, K Caroline Selvi, P Ganesh, P Padmanabhan, Mohammed Ali.

Department of Medical Gastroenterology, Madras Medical College, Chennai 600 003, India

Introduction: Though vascular complications are commonly seen in chronic pancreatitis, cavernous transformation of portal vein or portal cavernoma is uncommon. Here we report a case of acute on chronic pancreatitis with vascular (portal cavernoma) and endocrine complications. 
Case Summary: 42 year old male came with $\mathrm{h} / \mathrm{o}$ upper abdominal pain for 3 months. Pain was in the epigastrium, deep boring, aggravated by food, and was relieved on bending forward. On examination, abdomen was soft and non tender. He was a chronic alcoholic and smoker. Investigations showed very high blood sugar $(500 \mathrm{mg} / \mathrm{dl})$. USG abdomen showed enlarged diffusely hypoechoic pancreas with specks of calcification and MPD dilatation. CECT abdomen showed calcifications in the head, body and tail of pancreas. Portal Doppler showed cavernous transformation of portal vein with multiplecollaterals. Conclusion: Vascular complications like splenic vein thrombosis, pseudoaneurysm though common this case presented with rare complication of portal cavernoma and endocrine complication.

\section{P4}

\section{A rare case report of noninsulinoma pancreatic hypoglycemia syndrome (NIPHS) in an adult due to localised islet cell hyperplasia successfully managed with enucleation}

M K Daga, N Sinha, H K Nayak, R Kumar, $N$ Raizada, A Sothwal, R Bajaj.

Department of Medicine, Maulana Azad Medical College, Delhi 110 002, India

Persistent hyperinsulinemic hypoglycemia is caused most commonly by an insulinoma in adults or by nesidioblastosis in neonates. In adults, localized islet cell hyperplasia is a rare disorder. We present the case of a previously healthy 45 -yearold woman with recurrent episodes of altered sensorium. She presented with a similar episode to our emergency. Apart from altered sensorium her other examination was essentially normal. Laboratory findings revealed a low fasting blood glucose level $(15 \mathrm{mg} / \mathrm{dL})$, and a high insulin level (847 muIU/ $\mathrm{mL}$ ) with an insulin:glucose ratio of 58 . A â€œ72 hr fastâ€• test confirmed the diagnosis of hyperinsulinemic hypoglycemia. Abdominal imaging by various modes was essentially normal. Endoscopic ultrasound revealed a hyperechoic area in the head of the pancreas. Subsequent investigation by stimulation of the pancreas through cannulation of the pancreatic vascular supply revealed marked insulin release to low level calcium challenge across head regions of pancreas. Based upon these investigations, the patient underwent enucleation of the uncinate process alongwith a part of pancreas. Intraoperative ultrasonography of rest of pancreas was normal. Histological examination revealed hyperplasia of Islet cells. On immunostaining, most of the islet cells showed reactivity to insulin. Histology was consistent with the diagnosis of localized islet cell hyperplasia leading to persistent hyperinsulinemic hypoglycemia. The patient is currently doing well. Localized islet cell hyperplasia with such a very high insulin level is exceedingly rare in adult populations and has never been reported in literature. Partial pancreatectomy can be a good treatment option for such a condition.

\section{P5}

\section{Unusual cystic lesions of pancreas}

Biggs Saravanan Ramachandran, Mohammed Ali, P Padmanabhan, P Ganesh, K Prem Kumar, Caroline Selvi, Kani Sheik, B Vinoth, M Malarvizhi.

Department of Medical Gastroenterology, Madras Medical College, Park Town, Chennai 600 003, India

We report three unusual cystic lesions of pancreas namely Solid cystic tumor of pancreas, Lymph cyst of pancreas and pancreatic pseudocyst presenting as Cysto gastric fistula. Solid cystic tumor or Frantz tumor of pancreas accounts for less than $1 \%$ of pancreatic tumors and it is common in young females in their second decade unlike other pancreatic tumors. We diagnosed this case using preoperative Endoscopic ultrasonography (EUS) guided fine needle aspiration (FNA). Surgical enucleation was planned based on this and done with out any recurrence of the disease. Lymph cysts or chylous cysts of pancreas have never been reported before but retroperitoneal chylous cysts attached to pancreas have been reported. We diagnosed and followed this patient with serial EUS and FNA without any recurrence. Thirdly spontaneous rupture of pancreatic pseudocyst in to the stomach (cysto gastric fistula) is observed in less than $5 \%$ of pancreatic pseudocysts in their natural history. While we were planning for endoscopic cystogastrostomy we found fistulous communication between pseudocyst and stomach with reduction in abdominal mass clinically. Pseudocyst resolved without any sequelae. We report these cases since they are very rare to find in clinical practice and physicians should be aware of these possibilities when they encounter cystic lesions of pancreas.

\section{P6}

Genetic polymorphism in tumor necrosis factor alpha (TNF-Alpha -308G/A and -238G/A) gene and risk of pancreatic adenocarcinoma

Istaq Ahmad, ${ }^{1,2}$ Mohammad Asim, ${ }^{1}$ Sunil $K$ Polipalli, ${ }^{1}$ Anil Kumar Agarwal, ${ }^{2}$ P Kar. $^{1}$

${ }^{1}$ Department of Medicine, PCR Hepatitis Laboratory, Maulana Azad Medical College, University of Delhi, New Delhi 110 002, ${ }^{2}$ Department of GI Surgery, G B Pant Hospital, Maulana Azad Medical College, New Delhi 110 002, India

Background and Aim: Adenocarcinoma of exocrine pancreas is the fourth leading cause of cancer related death in 
the world. Polymorphisms of the TNF gene have been related to TNF production and outcome in a variety of inflammatory and malignant diseases. Proinflammatory cytokines and the inflammatory state appear to affect outcome in pancreatic cancer. Thus, the present study examined the possibility of using TNF -308 and -238 polymorphisms as a prognostic marker of pancreatic cancer. Methods: Two polymorphisms located in the promoter region (positions $\hat{\mathrm{a}}^{\wedge} 308$ and $\hat{\mathrm{a}}^{\wedge} 238$ ) in TNF- $\alpha$ gene were determined using PCR-RFLP based method. Out of 30 pancreatic cancer, 11 patients were female $(36.66 \%)$ and 19 patients were male $(63.33 \%)$ with a mean age of $47.42 \pm$ 11.32 years (range $22-70$ years). In control group 20 were male and 10 were female with an average age of $36.75 \pm$ 11.75 years. Odds ratios (ORs) and 95\% confidence intervals (CI) were estimated using logistic regression analysis adjusted for age, sex, smoking and BMI.

Results: The frequencies of TNF-A polymorphisms were both similar in patients with pancreatic adenocarcinoma patients and in controls $\left(\mathrm{OR}=0.84 ; 95 \% \mathrm{CI}\right.$ : $0.22 \hat{a}^{\wedge} 3.13$ for $\mathrm{TNF} \pm 308$ and $\mathrm{OR}=0.46$; $95 \%$ CI: $0.13 \hat{a}^{\wedge} 1.59$ for TNFAầ' 238 ).

Conclusion: We suggest that both SNPs of TNF-A not genetic risk factor for PA susceptibility and may not play an important role in pancreatic cancer. Other cytokines may be of more important than TNF in determining the inflammatory state and disease progress in pancreatic cancer which may be validated with larger studies.

\section{P7}

\section{Genotyping of glutathione-S-transferase and its role in pancreatitis: SGPGI experience}

\author{
Divya Singh, Shweta Singh, ${ }^{*}$ Gourdas Choudhuri, \\ Sarita Agarwal. ${ }^{*}$
}

Departments of Gastroenterology and *Genetics, Sanjay Gandhi Postgraduate Institute Medical Sciences, Lucknow 226014 , India

Background and Aim: Glutathione-S-transferases (GST), a family of phase II detoxification enzymes, provide protection against toxic products generated by reactive oxygen species. The mutated alleles of genes encoding enzymes metabolizing these substances and their metabolites may be disease modifiers. Thus the study was aimed to compare the genotype frequencies of GSTM1 and GSTT1 and GSTP1 genetic polymorphism in patients with chronic pancreatitis and healthy subjects.

Method: Blood samples were taken from 147 patients of tropical calcific pancreatitis (TCP, $n=75$ ), alcoholic chronic pancreatitis (ACP, $n=72$ ) and healthy controls (HC, $n=$ 100). DNA extraction was done manually. GSTT1 and M1 genotyping was done by multiplex PCR. GSTP1 genotyping was done by PCR-RFLP.
Result: The frequency of variant genotype of GSTP1 was significantly high in TCP group (70.6\%) in comparison to HC $[p=0.03 * ; \mathrm{OR}=1.9(1.1-3.7)]$ and ACP groups $[p=0.04 *$; $\mathrm{OR}=2.7(1-6.2)]$. GSTP1 polymorphism was associated with TCP. The frequency distribution of GSTM1 functional and null genotype did not differ in TCP $(p=0.7)$ and ACP groups ( $p=0.2)$ in comparison to HC group $(67 \%$ and $33 \%$ ). Similarly no significant differences were observed with GSTT1 functional and null genotype in TCP $(p=0.4)$ and ACP $(p=0.2)$ groups in comparison to HC. When we clubbed TCP and ACP group we did not find any significant difference between patient and control groups.

Conclusion: This study provides evidence that only GSTP1 polymorphism is associated with TCP.

\section{P8}

\section{Acute pancreatitis associated with acute hepatitis E:} A series of ten patients

Mithun Raj, Samir Mohindra, C Vasudev, Rakesh Aggarwal, Vivek Saraswat, Uday C Ghoshal, Gour Choudhuri.

Department of Gastroenterology, Sanjay Gandhi Postgraduate Institute of Medical Sciences, Raebareli Road, Lucknow 226 014, India

Introduction: Acute pancreatitis (AP), an uncommon association with acute viral hepatitis, has been reported with hepatitis A or B and rarely with E. We present a series of 10 patients with AP associated with hepatitis E.

Methods: Hospital records of patients admitted with AP to a tertiary institute (May 2004-April 2010) were evaluated. Acute hepatitis E was diagnosed biochemically with presence of IgM antibody against the virus in serum. AP was diagnosed by high serum amylase or lipase ( $>3$ times upper limit of normal) and imaging (abdominal ultrasonography and/or computerized tomography) and severity was assessed by Atlanta criteria. Other common causes of AP were excluded by clinical evaluation and appropriate investigations.

Results: Of 724 patients with AP, 10 (1.4\%; median age 27.5 years, range 16 to 54,9 male) had hepatitis $E$ and no other cause for AP. Median interval between onset of hepatitis and AP was 7 days (range 0-24). 6 patients had mild and 4 severe AP. Complications included intra-abdominal collections (4), ARF (2) and acute respiratory distress syndrome (1). 4 patients had pleural effusion and 2 had transient ascites. None needed surgical, endoscopic or percutaneous intervention. Median bilirubin level was $9.6 \mathrm{mg} / \mathrm{dl}$ (3.5-15.4) and ALT $910 \mathrm{IU} / \mathrm{L}$ (54 to 4009). All xpatients recovered completely with conservative measures. Median duration of hospital stay was 9 days (range 3-25).

Conclusion: AP associated with hepatitis E is not uncommon and most recover from the disease. Awareness about 
such an association is needed in tropical countries where hepatitis $\mathrm{E}$ is endemic.

\section{P9}

\section{MCP-1-2518 (A/G) and IL-8-251 (A/T) are not associated with acute pancreatitis in Indian population}

S Siva Prasad, ${ }^{1}$ Manu Tandon, ${ }^{2}$ Rajesh Gupta, ${ }^{1}$ L Sandeep, ${ }^{2}$ $P$ Nitesh ${ }^{2} R$ Mohan. ${ }^{2}$

${ }^{1}$ Asian Health Care Foundation, ${ }^{2}$ Asian Institute of Gastroenterology, Somajiguda, Hyderabad 500 082, India

Introduction: Chemokines can attract leukocytes to the site of injury. They play vital role in inflammatory reaction. Monocyte chemoattractant protein-1 (MCP-1) and interleukin-8 (IL-8) are typical examples for it. MCP-1 and IL-8 gene polymorphisms were reported to be associated with acute pancreatitis. Thus the present study was taken up for the first time to asses the possible association between the polymorphisms of MCP-1-2518 A/ G, IL-8-251 A/T, SPINK1, PRSS1 genes and Acute Pancreatitis in Indian Population.

Methods: Study was included 83 patients diagnosed for acute pancreatitis and 85 controls. MCP-1-2518 polymorphism was genotyped by PCR-RFLP method. IL-8-251 A/T was analyzed by allele specific PCR and confirmed by sequencing where as SPINK1 and PRSS1 polymorphisms were accessed by direct sequencing.

Results: Out of total number, $32(36.36 \%)$ patients were wild type, 49 (58.1\%) were heterozygous and 4 (5.4\%) homozygous mutants for MCP-1-2518 polymorphism. The distribution of IL-8-251A/T alleles was as follows: AA; 26 (31.3\%), AT; 19 (22.8\%), and TT; 38 (45.8\%). The case-control analysis of genotype of MCP-1$2518 \mathrm{~A} / \mathrm{G}$ and IL-8-251 A/T polymorphisms showed no association with acute pancreatitis. In this study no patient presented SPINK1, PRSS1 polymorphism. Thus the present study concludes that MCP-1-2518A/G polymorphism is not associated with Acute pancreatitis in Indian population unlike other Asian and American studies.

\section{P10}

\section{Estimation of ductal epithelial cells differentiating into insulin cell like clusters in human pancreas}

$G$ Radhika, ${ }^{1}$ P Pavan Kumar, ${ }^{1} M$ Sasikala, ${ }^{1} G V$ Rao, ${ }^{1}$ D Nageshwar Reddy. ${ }^{2}$

${ }^{1}$ Asian Health Care Foundation, ${ }^{2}$ Asian Institute of Gastroenterology, Somajiguda, Hyderabad 500 082, India
Background: Autologus sources of stem/ progenitors and pre existing $\hat{\mathrm{I}}^{2}$ cells are currently under investigation for expansion and differentiation into insulin producing cells. Pancreatic ductal epithelium, gall bladder, liver, biliary epithelium, form newer sources for pancreatic endocrine lineages since their ontogeny during embryogenesis is similar. The aim of the present study is to estimate the number of pancreatic ductal progenitors available to be induced to differentiate into insulin producing cells.

Methods: Pancreatic ducts of 12 patients undergoing pancreatic surgeries were freed of the exocrine tissue, sectioned and immunostained for CK19, Insulin and imaged using Bioimager. CK19+Cells co expressing Ki67 and insulin positive cells were counted in the same location. Number of ICCs that result from the proliferation of and differentiation of PDECs is counted and calculated using simple mathematics.

Results: Counting of ducts for proliferating CK19+PDEC in 10 locations indicate the occurrence of $27 \pm 9$ cells/field in a field view of $10 \times$ magnification with a field number of $22 \mathrm{~mm}$. Therefore 3600-7200 PDECs would be expected in $1 \mathrm{~cm}$ sections. 1600-1800 ICCs may differentiate from 54000 progenitors in a duct of $10 \mathrm{cms}$ at the rate of 32 cells requirement in a time frame of 48-72 hrs, under normal physiological conditions.

Conclusion: Approximately 0.05 million -0.1 million PDECs may be proliferating to differentiate into ICCs. As per this study, $1 \mathrm{~cm}$ section of the ductal tissue may give rise to 100 ICCs.

\section{P11}

\section{Alterations in plasma amino acid levels in chronic pancreatitis}

B N Girish, ${ }^{2}$ G Rajesh, ${ }^{1}$ K Vaidyanathan, ${ }^{3}$ V Balakrishnan. ${ }^{1}$ Departments of Gastroenterology, ${ }^{1}$ Physiology, ${ }^{2}$ and Biochemistry, ${ }^{3}$ Amrita Institute of Medical Sciences, Kochi

Background: Dietary proteins and amino acids can modulate pancreatic function. We aimed to estimate the levels of plasma amino acids in chronic pancreatitis patients and study relation with disease characteristics as well as exocrine and endocrine insufficiency.

Methods: Eighty-four patients with alcoholic chronic pancreatitis (ACP), 91 with tropical chronic pancreatitis (TCP) and 113 healthy controls were recruited. Disease characteristics and imaging features were recorded. Plasma free amino acid levels were estimated using reverse-phase high-performance liquid chromatography. Polyclonal antibody ELISA was used to assess pancreatic fecal elastase-1.

Results: The majority of the plasma free amino acid levels were decreased in $\mathrm{CP}$ patients, whereas glutamate, glycine, 
proline and lysine were elevated, as compared to controls. Multivariate logistic regression analysis revealed that decrease in branched chain amino acid concentration was significantly associated with presence of diabetes and low fecal elastase-1. In addition, significant positive correlation was observed between branched chain amino acids and pancreatic elastase-1 $(\mathrm{r}=0.724, p<0.001)$.

Conclusion: Alterations of plasma amino acid levels seen in $\mathrm{CP}$ suggest selective amino acid deficiencies and seem to correlate with exocrine and endocrine insufficiency. Alteration in sulfur containing amino acids and branched chain amino acids may play a key role.

\section{P12}

\section{Role of transabdominal ARFI and elastography in diagnosing pancreatic mass lesions}

P B S S Raju, ${ }^{1}$ M A Mateen, ${ }^{2}$ Majaz Hussain, ${ }^{2}$ G V Rao, ${ }^{3}$ D N Reddy. ${ }^{1}$

${ }^{1}$ Department of Gastroenterology, ${ }^{2}$ Department of Radiology, ${ }^{3}$ Department of Surgical Gastroenterology, Asian Institute of Gastroenterology, Hyderabad 500 082, India

Objective: To evaluate feasibility of transabdominal elastography in patients with pancreatic mass lesions to differentiate between benign and malignant pathology.

Methods: Patients with pancreatic mass lesions were prospectively studied. Images were recorded using Seimens acuson 2000 ultrasound machine. eSie touch software was used for elastography and color code was given accordingly. Initially twenty healthy volunteers were studied to obtain the color coding for normal pancreas. 15 patients with pancreatic mass lesions were included and color coding was given and compared with normal pancreas. Normal healthy pancreas has green color, red color indicates hard consistency and yellow and orange colors were intermediate between green and red.All these lesions were confirmed on FNAC/biopsy

Results: Of the 15 patients, six had benign masses with greenish to yellow suggestive of soft consistency and 6 had hard lesions with orange to red color coding suggestive of malignant lesions. FNAC from soft lesions confirmed benign pathology, 2 patients had neuroendocrine tumor and 4 patients had inflammatory pathology. Of the 6 hard lesions, two patients underwent surgery and biopsy showed adenocracinoma. Remaining three patients with hard lesions underwent FNAC confirmed malignant pathology. 3 patients had soft masses with hard component under went curative resection and was suggestive of mucinous cystadenoma.

Conclusion: Transabdominal elastography using eSie touch software is useful in diagnosing pancreatic mass lesions.

\section{P13}

Dynamic nature of organ failure in acute pancreatitis and its outcome

$R B$ Thandassery, $R$ Kochhar, T D Yadav, A D Galle, $U$ Dutta, K Singh.

Department of Gastroenterology and General Surgery,

* Postgraduate Institute of Medical Education and Research, Chandigarh 160 012, India

Objective: To compare the clinical course and outcome of patients with transient organ failure (TOF), (lasting for $48 \mathrm{hrs}$ ) in 1 st week of admission among those with acute pancreatitis (AP).

Methods: Consecutive patients with AP (January 2009June 2010) were evaluated for organ failure (OF), its severity and dynamics during 1st week of admission. Modified multiple organ failure score (MOFS) was used to identify and grade severity of OF. Statistical analysis was done with SPSS version 16.

Results: Of the 81 patients, mean age 40.1 years (range 13 to 84), 55 males; 21 (26\%) patients had no OF, 26 (32\%) had TOF and $34(42 \%)$ had POF in the 1 st week. Between POF and TOF; former had longer hospital stay $(20.6 \pm 2.2$ and $13.1 \pm 2.2$ days, $p=0.055)$, longer ICU stay $(12.32 \pm 2.1$ and $0.35 \pm 0.27$ days, $p=0.000)$, more infections $(23 ; 67.6 \%$ and $4 ; 15.3 \%$, $p=0.000)$, more infected necrosis $(9 ; 90 \%$ and $1 ; 10 \%, p=$ $0.02)$, more local complications $(32 ; 94 \%$ and $20 ; 77 \%, p=$ 0.142 ), more requirement for percutaneous drain placement $(16 ; 47 \%$ and $5 ; 26 \%, p=0.042)$, more requirement for surgery $(10 ; 29.4 \%$ and $2 ; 7.6 \% p=0.01)$, more requirement for ventilatory support $(0 \%$ and $12 ; 35.3 \% p=0.000)$ and more mortality $(0 \%$ and $13 ; 38 \% p=0.001)$. By logistic regression analysis, APACHE II Score (at $48 \mathrm{hrs),}$ worsening of MOFS and infected necrosis were independent predictors of mortality.

Conclusion: Subclassification of patients with AP into TOF and POF predicts clinical course and outcome. Patients with POF have poor outcome and prolonged course as compared to those with TOF.

\section{P14}

\section{Hypotension in 1st week of acute pancreatitis predisposes to development of infected pancreatic necrosis}

R B Thandassery, R Kochhar, T D Yadav, ${ }^{*}$ P Dixit, U Dutta, $K$ Singh.

Department of Gastroenterology and General Surgery,* Postgraduate Institute of Medical Education and Research, Chandigarh 160 012, India 
Objective: To correlate the occurrence of hypotension (CVS failure), renal failure, respiratory failure CNS failure and coagulopathy in the first week of acute pancreatitis (AP) with subsequent development of infected necrosis (IN).

Methods: Consecutive patients with AP (January 2009 and June 2010) were evaluated for organ failure (OF) and its severity in the first week of admission. Modified multiple organ failure (Modified Bernard score) score was used to identify and grade severity of OF. The occurrence of IN (isolation of bacteria in necrosectomy specimen or CT guided FNAC of pancreatic necrosis) was compared between groups with OF and without OF. Statistical analysis was carried out with SPSS version 16 .

Results: Of the 81 patients, mean age 40.1 years (range 13 to 84), 55 males; 60 (74\%) patients had OF. 13 (16\%) patients (total 15 isolates, all of enteric origin) had IN (67\% Gram negative). Occurrence of IN was not significantly different between patients with OF (18.3\%) and without OF (14.3\%), $p=0.48$. However IN occurred in $11.7 \%$ of patients with and $28.6 \%$ patients without hypotension respectively, $p=0.04$. Rest of the organ systems analysed did not show any significant difference in occurrence of infected or sterile necrosis. IN was present in $13.3 \%$ patients without and $19.4 \%$ with respiratory failure, $p=0.32$. Renal failure, coagulation failure and CNS failure also did not differ in patients with or without IN; $p=0.32,0.14$ and 0.41 respectively.

Conclusion: Hypotension in the first week of AP is a risk factor for development of IN.

\section{P15}

\section{Effect of oral glutamine supplementation on gut permeability and endotoxaemia in severe acute pancreatitis: A randomized controlled trial}

S K Mishra, Namrata Singh, Vikas Sachdev, Y K Joshi, Anoop Saraya.

Department of Gastroenterology and Human Nutrition, All India Institute of Medical Sciences, New Delhi 110 029. India

Background: Gut permeability is altered in severe acute pancreatitis (SAP) resulting in bacterial translocation and increased morbidity and mortality. Glutamine improves the immune, inflammatory and metabolic functions and outcome.

Aim: To study the effect of oral glutamine versus whey protein in SAP on gut permeability and endotoxaemia.

Methods: All consecutive patients of SAP admitted in first week of illness were randomized into two groups and given $20 \mathrm{gm} /$ day of glutamine (intervention) or whey protein (control) for 7 days. The primary outcome was to evaluate changes in intestinal permeability (assessed by means of Lactulose/Mannitol (L/M) excretion in urine) and endotoxaemia (assessed by endotoxin-core antibodies type $\operatorname{IgG}$ and $\operatorname{IgM}$ (Endocab $\operatorname{IgG}$ and $\operatorname{IgM}$ )), which were done just prior to and 7 days after intervention. Secondary outcome measures were infectious complications, total hospital/ICU stay, mortality, CRP and prealbumin levels.

Results: From Sept 2008 to May 2010, 44 patients of SAP were randomized in the two groups (22 patients in each). There was no change in gut permeability after intervention. However the endocab IgM levels increased significantly (39 (2-175) to $62(10-350) \mathrm{GMU} / \mathrm{ml}, p=0.033)$ and CRP levels decreased significantly (190 (1-287) to $124(1-267)$ $\mathrm{ng} / \mathrm{ml}, p=0.039)$ in the glutamine group. There was no change in pre albumin and endocab $\operatorname{IgG}$ levels in the glutamine group. No differences were observed in hospital / ICU stay, infectious complications and mortality in both the groups.

Conclusions: Oral glutamine supplementation did not change the gut permeability. However it may have a role in decreasing inflammation and endotoxemia in SAP, but a larger study is needed to substantiate.

\section{P16}

\section{Interferon gamma is a potent inhibitor of expression and nuclear translocation of homeodomain transcription Factor Pdx1 causing reduced $\hat{\mathbf{I}}^{2}$ cell area in chronic pancreatitis}

P Pavan Kumar, ${ }^{1}$ M Sasikala, ${ }^{1}$ G Radhika, ${ }^{1}$ Manu Tandon, ${ }^{2}$ $G$ V Rao, ${ }^{2}$ D Nageshwar Reddy. ${ }^{2}$

${ }^{1}$ Asian Health Care Foundation, ${ }^{2}$ Asian Institute of Gastroenterology, Somajiguda, Hyderabad 500 082, India

Background and Objectives: Reduced pancreatic volume and $\hat{\mathrm{I}}^{2}$ cell area leading to diabetes were demonstrated to be associated with chronic pancreatitis (CP). Inflammatory Cytokines are proposed as potent inhibitors of $\mathrm{Pdx} 1$ expression, insulin gene transcription and $\hat{\mathrm{I}}^{2}$ cell apoptosis. Since CP is an inflammatory condition and pdx 1 plays an important role in development of pancreas, the function of which is related to its translocation into the nucleus, the present study was conducted to identify the potential inhibitors of Pdx1 expression and translocation.

Methods: Pancreatic tissue explants from controls (without $\mathrm{CP})$ were subjected to cytokine $(100 \mathrm{pg} / \mathrm{ml})$ exposure for $1 \mathrm{hr}$ in high glucose medium. Pdx1 localization in $\mathrm{CP}$ and cytokine exposed tissues was assessed by IHC and nuclear expression was confirmed by western blot. $\hat{\mathrm{I}}^{2}$ cell area was measured from the images taken using CARVII bioimager and quantified employing IP Lab software. 
Results: All the cytokines caused reduction in the translocation of Pdx1 into nucleus. While TNFA and IL6 caused $40 \%$ reduction, IFN $\gamma$ exposure resulted in $60 \%$ reduction in translocation of Pdx1.Anti inflammatory IL10 addition into the cultures improved translocation by $10 \%$ but complete reversal was not achieved. $\mathrm{CP}$ islets also showed significant $(P<0.01)$ reduction of nuclear localization of $\mathrm{pdx} 1 . \hat{\mathrm{I}}^{2}$ cell mass in $\mathrm{CP}$ was reduced by $31 \%$ and cytokines exposed islets showed $26 \%$ reduction.

Conclusion: Interferon gamma was found to be a potent inhibitor of nuclear expression of Pdxland correlates with decreased $\hat{\mathrm{I}}^{2}$ cell area in chronic pancreatitis.

\section{P17}

\section{Predictors of mortality in severe acute pancreatitis}

M K Hinduja, T D Yadav, R Kochhar, A Agrawal, $R$ Singh. Departments of General Surgery and Gastroenterology, Postgraduate Institute Medical Education and Research, Chandigarh 160 012, India

Aims: To determine predictors of mortality in SAP.

Methods: 66 patients of SAP were studied prospectively between Jan 2009 and June, 2010 for clinical features, lab parameters, Ranson's and APACHE II scores and CT severity index factors affecting mortality were analyzed. All patients were managed with prophylactic antibiotics, nasojejunal/parenteral nutrition and supportive care. Necrosectomy was done for persistent organ failure, infected necrosis and clinical deterioration.

Results: Of the 66 patients (45 males, age 13-84 years) 21 underwent percutaneous catheter drainage, 31 alcohol, 24 gallstones, 9 others and 16 underwent necrosectomy. 16 patients died, of whom 12 had been operated. On univariate analyzed factors associated with mortality were obesity $(p=0.001)$, number of comorbidites $(p=0.001)$, duration of pancreatitis $(p=0.001)$, presence of low $(p=0.001)$ and base deficit $(p=0.001)$ CT severity index $>7 \quad(p=0.001)$, extent of necrosis $(p=0.001)$ at admission. Ranson's score at $48 \mathrm{~h}(p=0.001)$, APACHE II score at admission $(p=0.001)$, number of organ failures $(p=0.001)$, requirement of surgery $(p=0.001)$ and postoperative enteric fistula $(p=0.001)$. On multivariate analysis, base deficit serum bilirubin and need for surgery were independent predictors of mortality.

Conclusion: Factors at admission correlating with mortality were comorbidity, APACHE II and CTSI score, multiorgan failure high serum bilirubin and high base deficit. Patients managed conservatively survived more often than those operated.

\section{P18}

Validation of pancreatitis outcome prediction (POP) score in patients with severe acute pancreatitis-A pilot study

Sathish Kumar Akkim, Ajith Roni, Satyashree, Pavan Kumar Reddy, Lalit Nihal.

Department of Medical Gastroenterology and Hepatology, Narayana Medical College, Nellore

Background: Severe acute pancreatitis carries high mortality and morbidity. Current prediction scores such as Ranson, CTSI cannot be applied immediately on admission. POP score which can be calculated on the day of admission predicted outcome more accurately.

Objective: Validation of pancreatitis outcome prediction score (POP) in patients with severe acute pancreatitis.

Design: This prospective study was conducted in a tertiary care teaching hospital. Study period was from September 2009 to July 2010.

Methods: Patients aged $>18$ years with the diagnosis of severe acute pancreatitis were included in the study. These patients were prospectively evaluated by using POP score to assess the mortality. POP score has 6 variables; Arterial $\mathrm{pH}$, Age, Serum Urea, Mean arterial pressure, Pao2 / Fio2 ratio, and total serum calcium. Each variable had scores from $1-10$, with a maximum score of 40 . The mortality rate is $10 \%$ when POP score is 10 and increases to $90 \%$ when score is 30 .

Results: During the study period 72 patients (Males 51,Mean Age 29.8 yrs)were admitted with acute pancreatitis, 21 of them had severe acute pancreatitis and 5 patients died. POP Score between $0-10$ there was no mortality, 11-20 there was 50\% mortality and scores more than 21 there was $100 \%$ mortality. Conclusion: POP score predicted mortality accurately in patients with severe acute pancreatitis. This is a one time score which can be calculated within $24 \mathrm{hrs}$ of admission with easily available variable.

\section{P19}

Clinical profile and follow up of patients undergoing lateral pancreaticojejunostomy for chronic calcific pancreatitis

S Shareej, Varghese Thomas, V K Prathapan. Departments of Medical and Surgical Gastroenterology, Calicut Medical College, Kerala

Aim: To assess the clinical profile and short outcome of patients managed by Lateral Pancreaticojejunostomy (LPJ) for control of severe abdominal pain. 
Method: Retrospective analysis of the records and follow up of patients from March 2008 to July 2010.

Results: Total number $=28$, mean age 39.89 (range 20-65). M: F ratio 3.6:1. 16 (57.1\%) had diabetes. Mean pancreatic duct diameter was $8.1 \mathrm{~mm}$. CA 19-9 were done for 13 cases, of these, 11 cases $(73 \%$ ) had values $>37$ unit $/ \mathrm{ml}$ (median 114.23 ). Patients with malignancy had higher mean Ca19-9 (122.25) levels compared to benign cases (110.6) but this was statistically insignificant. Average insulin requirement before and after surgery were 35.18 and 24.62 (p.004) respectively. Pancreatic ductal biopsy was benign in $24(85.7 \%)$ and malignant in 4 cases. Two patients developed malignancy during follow up (one duodenal obstruction, other disseminated metastasis). Twenty one ( $(75 \%)$ became pain free on follow up. One patient lost to follow up. Patient with poor pain relief had malignancy. Three patients had duodenal ulcer underwent TV (truncal vagotomy) and GJ along with LPJ. During follow up all patients who underwent LPJ (25 cases) for CCP and CCP with DU (3 cases) had a better course. They had better diabetes control at 3rd and 6th months $(p=.003)$ and less insulin requirement $(p=.004)$.Six patients with malignancy died during follow up, median survival was 7 months (range 4-8 months).

Conclusions: In this study $75 \%$ had pain relief and better quality of life with fairly controlled diabetes and lesser insulin requirement following surgery. Symptom relief and survival of patients with malignancy were poor.

\section{P20}

\section{Expression of extracellular matrix degrading enzymes in patients with carcinoma of the pancreas}

Nidhi Singh, Prasenjit Das, Siddharth Srivastava, Shyam Chauhan, ${ }^{* *}$ Siddharth Dattagupta, ${ }^{*}$ Anoop Saraya. Departments of Gastroenterology and Human Nutrition, *Pathology and ${ }^{* *}$ Biochemistry, All India Institute of Medical Sciences, New Delhi 110 029, India

Background: Pancreatic cancer is the 5th leading cause of cancer related death world-wide. Overall prognosis is poor even after curative resection. Cathepsin-L and MMP-2 (Matrix Metalloprotease-2) are proteases that degrade the peri-tumoral stroma and help in tumor dissemination and thus used as indicators of tumor behavior. We aimed to assess cathepsin- $\mathrm{L}$ and MMP-2 expression in patients with pancreatic cancer.

Methods: Paraffin embedded tissue from 29 operated specimens of carcinoma pancreas were analyzed for immunohistochemistry (IHC) for the said markers. The protein expression pattern was noted both in terms of area of positivity (on slide) and intensity of expression, both in the tumor and the stromal fibroblasts. Intensity of expression was scored in comparison to the intensity of control biopsies. Semi-quantitative scoring was done in both of these components. Clinical data were analyzed with the pattern of immuno-reactivity. The relationship of cathepsin- L and MMP-2 to clinicopathological parameters was evaluated using Student t-test.

Results: For cathepsin-L, immuno-reactivity was in $90 \%$ cases (cytoplasmic). For MMP-2, immuno-reactivity was 95\% (cytoplasmic and membranous). Stromal fibroblasts in and around the tumor, showed a wide area distribution with high stain intensity for both the markers. Though no other clinical correlation was found, for cathepsin-L, there was a significant correlation to the presence of retroperitoneal lymph nodes $(p<.05)$.

Conclusion: Cathepsin L and MMP-2 had a strong expression in tumor cells as well as stromal fibroblasts in and around tumor. A significant correlation of cathepsin- $\mathrm{L}$ with presence of retroperitoneal lymph node suggests its role in progression of the disease.

\section{P21}

\section{Role of TGF $\beta 2$ and Smad3 in the induction of fibrosis in chronic calcific pancreatitis and pancreatic adenocarcinoma}

\section{K Shymi, H Devaraj, R Surendran, V Vimalraj, R Prabhakar, $V$ Jayanthi. \\ Department of Biotechnology, University of Madras and Department of Gastroenterology, Stanley Medical College, Chennai 600 003, India}

Pancreatic adenocarcinoma is one of the most fatal malignancies. Intensive investigation of molecular pathogenesis might lead to identifying useful molecules for diagnosis and treatment of the disease. Like chronic pancreatitis, adenocarcinoma of the pancreas, also has a remarkable fibrotic component (desmoplastic reaction).

Aim: To examine the role of TGF $\beta 2$ and Smad 3 in the induction of fibrosis in surgical specimens of chronic calcific pancreatitis (CCP) and pancreatic adenocarcinoma. Immunohistochemistry, Immunofluorescence and Western Blotting techniques were used to understand the expression of TGF $\beta 2$ and Smad 3 and Sirius red staining was used to detect the level of accumulation of collagen.

Results: The level of collagen accumulation was high in CCP and pancreatic adenocarcinoma compared to normal. An overall increase in the intensity of TGF- $\beta 2$ staining was detected around ductal cells and pancreatic acinar cells in CCP and pancreatic adenocarcinoma compared to normal tissues. The amount of collagen accumulation correlated directly with the levels of expression of TGF-beta 2 protein in both the specimens. Cytoplasmic localization of $\operatorname{Smad} 3$ was low in $\mathrm{CCP}$ and pancreatic adenocarcinoma with higher nuclear mislocalization compared to normals.

Conclusion: TGF $\beta 2$ induces fibrosis in both CCP and pancreatic adenocarcinoma through $\mathrm{Smad} 3$ signaling pathway. 


\section{P22}

Endotherapy in pancreatic ascites-A study in 21 patients

Aditya Satyaprasanna, Mohammed Akbar, L R S Girinadh, P Murali Krishna.

Department of Gastroenterology, Andhra Medical College, Visakhapatnam, India

Aims: To study the clinical profile and short term outcome of endotherapy in patients with pancreatic ascites.

Methods: A total number of 21 patients with pancreatic ascites were recruited in the study. Patients who did not improve within 3 week of conservative treatment were subjected to pancreatic sphincterotomy with or without Stent placement. Therapeutic success was defined as clinical resolution of ascites and or fistula.

Results: All patients included were male with age range of 15 to 58 years. Etiology in 20 patients was alcohol related, in 1 patient it was unknown (chronic pancreatitis -15, acute pancreatitis-6, with pseudocyst- 4 , with effusion -3 ). Of the 21 patients, 6 improved with conservative management for 3 weeks, one patient died. 14 patients were subjected to ERCP. ERCP was successful in 10 cases. Duct disruption was identified in 13 cases (body-5, tail-7, genu-1). Of the 3 cases with acute pancreatitis that underwent endotherapy 1 improved. Of the 11 cases of chronic pancreatitis that underwent ERCP, 9 improved. Sphincterotomy was done in all, stent was placed in 6 cases. All the patients with stent improved. Of the 8 patients with only sphincterotomy, 4 improved. Of the 4 cases with pseudocyst, 2 improved with sphincterotomy and stent, 1 required surgery and the other died. Of the 3 cases with associated pleural effusion, 1 improved with conservative management, 1 with sphincterotomy, 1 with sphincterotomy and stent.

Conclusions: Pancreatic endotherapy offer an excellent option for management of pancreatic ascites. Sphincterotomy with stent was more successful than sphincterotomy alone.

\section{Surgical Gastroenterology}

\section{SG1}

\section{Malignancy of the duodenojejunal junction : A case series}

Anuj Bhatia, Vaibhav Pandey, Vivek Srivastava, Somprakas Basu, Anand Kumar and Vijay Kumar Shukla.

Department of General Surgery, Institute of Medical Sciences, Banaras Hindu University, Varanasi 221 005, India

Adenocarcinoma of the duodenum is a rare condition and represents only $0.3-0.4 \%$ of all gastrointestinal tract cancers with the fourth part constituting about $10 \%$ of these. We report a series of 6 cases of duodenojejunal junction malignancy over a period of eight years and discuss the clinical features, investigations, treatment provided and their outcome.

The mean age was 56.4 years and the mean duration of symptoms was 4.4 months. The presenting complaints were upper abdominal pain (83\%), loss of appetite and weight $(100 \%)$, postprandial vomiting $(33 \%)$, dehydration and dyselectrolemia (17\%). None had history of hematemesis/ melena/peptic ulcer disease. The mean BMI and $\mathrm{Hb}$ were 19.04 and $8.26 \mathrm{~g} / \mathrm{dL}$ respectively. All had similar picture in barium series suggestive of a mechanical obstruction in the third part of deuodenum (D3); proximal duodenal dilation, sudden cut off at D3 and delayed emptying. Gastroduodenoscopy was in 5 patients and it could see up to D2, which was unremarkable. The mean aorto-mesenteric angle and the aorto-mesenteric distance were $33 \mathrm{o}$ and $12.6 \mathrm{~mm}$ respectively. On ultrasonography, a mass was seen in the distal duodenum in $3(50 \%)$ patients, only lymph nodes were seen in 2 patients and no abnormality was detected in 1 patient. None of them afforded a CT scan of the abdomen.

Preoperative FNAC was done in 2 patients. One was inconclusive and the other was suggestive of lymphoma. Intraoperatively the mean size of the tumor was $8.25 \mathrm{~cm} 2$. Complete resection with lymph nodal clearance could be done in 3 patients $(50 \%)$; in the rest a palliative gastrojejunostomy was done. Histopathology showed lymphoma (NHL) in one and the rest had adenocarcinoma. Four patients received adjuvant chemotherapy and 3 patients completed one year follow up. One patient died within 1 year of surgery ( 8 months) due to tumor recurrence and dissemination.

\section{SG2}

Gastrointestinal stromal tumors (GIST): Experience from a single surgical unit

Anuj Bhatia, Somprakash Basu, Mohan Kumar, Vijay Kumar Shukla.

Departments of General Surgery and Pathology, Institute of Medical Sciences, Banaras Hindu University, Varanasi 221005 , India

Objective: Gastrointestinal stromal tumors (GISTs) are the rare tumors of gastrointestinal tract but most common mesenchymal neoplasms of the GI tract. We intend to present clinical profile, diagnosis and management of these tumors.

Methods: Eleven cases of GIST managed in a single surgical unit over last 7 years were included in the study. Demographic profile of the patients and their clinical presentation was studied. Ultrasound and CT scan abdomen were the main investigations used for evaluation with 
Upper GI endoscopy and X-ray chest being other important investigations. Histopathology, Imprint cytology and Immunohistochemistry were used to confirm the diagnosis.

Results: Mean age at presentation was 47.9 years (3570 years). Mean duration of symptoms was 5 months (10 days -2 years). Eleven patients had pain abdomen while 9 had abdominal lump. Two patients complained of vomiting, 1 had Upper GI bleed as the presenting symptom. Upper GI endoscopy was used to diagnose 3 cases of gastric GIST while USG and CT abdomen picked up 9 cases of ileal GIST. GIST was arising from ileum in 7 cases whereas 3 had gastric and 1 had mesenteric origin. Nine cases proved out to be low grade benign tumors while 2 had frank malignancy. Conclusion: Pain and lump abdomen are the most common clinical symptoms of GISTs. Vomiting, upper GI bleeding and weight loss are among the other common symptoms. Most of the tumors are benign and surgical resection remains the mainstay of treatment. Immunohistochemistry proved to be an important tool for the diagnosis.

\section{SG3}

\section{Surgery for fistula-in-ano; Types and treatment modalities}

\section{Isanka Nishan Gunarathna, D N Smamaraseker.} Department of Surgery, Faculty of Medicine, Colombo.

Introduction: The surgical treatment of fistula-in-ano is a major concern because of risk of recurrence and anal incontinence.

Aim: Aim of the study was to describe the types and treatment modalities.

Method: A total of 67 patients (mean age 39.54, range- 1183 years, male: 61 Female: 6) with cryptoglandular fistula in ano, who underwent surgery at the National Hospital of Sri Lanka, were prospectively studied. Statistical analysis was performed using the SPSS/Windows version 18.0 Results: The mean duration of follow up (from the definitive surgery) is 28.2 months (range 6-59 months). The distribution of fistulae was as follows: intersphincteric, 24 (35.8\%), transsphincteric, 39 (58.2\%), suprasphincteric $3(4.5 \%)$ and extrasphincteric none. A horseshoe extension and abcess occurred in $8(12 \%)$ and $15(23 \%)$ of the fistulae respectively. Treatment methods used were as follows. Cutting seton in 25 (37.3\%), fistulectomy/fistulotomy in 19 $(28.4 \%)$, coring the tract direct approximation of mucosa in $11(16.4 \%)$, fistula plug in $6(9 \%)$, mucosal advancement flap in $5(7.5 \%)$.

Conclusion: In our series Commonest type of fistulae was transsphincteric and the cutting seton was the treatment method. None of the patient got recurrences during follow up.

\section{SG4}

Spectrum of perforation peritonitis in single surgical unit of university hospital

Vivek Srivastava, Vaibhav Pandey," Mumtaz A Ansari," Anand Kumar. *

${ }^{*}$ Department of General Surgery, Institute of Medical Sciences, Banaras Hindu University, Varanasi 221 005, Uttar Pradesh

Background: Perforation peritonitis is a common surgical emergency in India and is a major cause of mortality and the spectrum differs from the west.

Objective: This study was conducted in a single surgical unit of University hospital to study the spectrum of perforation peritonitis and their outcome.

Results: A total of 133 cases of perforation peritonitis were admitted over three years duration. The data regarding the clinical presentation and postoperative course were collected and analyzed. The causes of perforation in our series was gastric in $46(34.5 \%)$, duodenal in $40(30 \%)$, enteric in 23 $(17.2 \%)$, appendicular in $8(6 \%)$, traumatic ileal in $9(6.7 \%)$ and tubercular in $7(5.2 \%)$ cases. The mean duration of presentation was 3.4 days, mean systolic blood pressure 94 . $\mathrm{mm}$ of $\mathrm{Hg}$, mean hemoglobin $8.6 \mathrm{mg} \%$, mean albumin $2.5 \mathrm{gm} / \mathrm{dl}$ and mean creatinine $1.47 \mathrm{mg} \%$. The mean operative time was $85.67 \mathrm{~min}$. The overall mortality was seen in 15 $(11.2 \%)$ cases and the overall morbidity in $66(50 \%)$ cases. The most common morbidities were surgical site infection $46 \%$ followed by wound dehiscence $28 \%$, chest infection $26 \%$, controlled leak $9 \%$, entero-cutaneous fistula $7 \%$, UTI $7 \%$, septicemia $6 \%$, ARF $6 \%$ and DVT $0.75 \%$.

Conclusion: In contrast to western literature, where lower GI perforations predominate upper GI perforations are common in India. In contrast to the common belief the gastric perforations are more common than duodenal perforation in Indian scenario. The mortality and morbidity after GI perforation is a significant burden which needs to be highlighted.

\section{Pediatric Gastroenterology}

\section{PG1}

Spectrum of esophageal mucosal lesions in pediatric celiac disease: a prospective controlled study

K K Prasad, B R Thapa, S Lal, C K Nain, A K Sharma, K Singh.

Department of Superspeciality of Gastroenterology, Postgraduate Institute of Medical Education and Research, Chandigarh 160 012, India 
Background: Celiac disease (CD) may cause changes throughout the gastrointestinal tract. It is not known whether the esophageal mucosa is involved in CD. The purpose of this prospective study was to evaluate the spectrum of mucosal damage to the esophagus in patients with $\mathrm{CD}$.

Methods: The spectrum of mucosal damage to the esophagus was investigated in 126 children with CD during 192 upper GI endoscopies performed to obtain duodenal biopsy specimens and was compared with findings in 49 children who underwent endoscopy for upper GI complaints without CD (non-CD patients) between January 1, 2009 and December 31, 2009. Children were diagnosed as cases of CD based on the modified ESPGHAN criteria and positive serology. Duodenal histologic lesions were classified as proposed by our group.

Results: The mean age of CD children (M:F::71:55) at presentation was $6.7+3.3$ years. $54 / 126(42.9 \%)$ of $\mathrm{CD}$ and $23 / 49$ (46.9\%) of non-CD patients showed normal esophageal mucosa. The prevalence of lymphocytic esophagitis (LE) was higher in CD (11.9\%) than non-CD (2.1\%) patients. The prevalence of eosinophilic esophagitis, follicular esophagitis, reflux esophagitis and non-specific esophageal mucosal changes were similar in both groups. All but one children with LE showed classic duodenal histologic lesion.

Conclusions: Our findings showed that the esophageal mucosa can be damaged in $C D$ patients and that the prevalence of LE is higher with CD. Awareness of potential coexistence of esophageal lesions in CD should promote optimal diagnosis of these conditions. Routine esophageal biopsies may be warranted when investigating for $\mathrm{CD}$

\section{PG2}

\section{Factors predicting neurological deterioration} after chelation therapy in patients with Wilson's disease

N Ravisankar Reddy, ${ }^{1}$ A S Puri, ${ }^{1}$ S Sachdeva, ${ }^{1}$ N Chaudhry, ${ }^{2}$ L Upreti. $^{3}$

${ }^{1}$ Departments of Gastroenterology, ${ }^{2}$ Neurology and ${ }^{3}$ Department of Radiology, GB Pant Hospital, Delhi

Background: There is paucity of information on the proportion of hepatic Wilson's patients who deteriorate on chelation therapy. There is also little information on factors that predict neurological deterioration. In this cohort study we intended to study what percentage of patients with hepatic Wilson's disease develop neuro deterioration on dpenicillamine. Our aim was to identify any prognostic factors which could predict neurological deterioration after the onset of chelation therapy.
Methods: We enrolled newly diagnosed Wilson's disease patients in the age group between 3-18 yrs who had hepatic or combined hepatic with neurological manifestations. We excluded fulminant Wilson's and other causes of chronic liver disease. Evaluation of liver status was done using Child, MELD score and New Wilson's scoring index. We studied putative predictive factors like age, gender, and mode of presentation i.e. hepatic versus combined hepatic plus neurological. All patients were followed up to $2 \mathrm{yrs}$ while on d-Penicillamine therapy.

Results: One patient had combined clinical neurological involvement along with hepatic manifestation (10\%). four of 10 patients ( $40 \%$ ) had CNS lesions lesions on MRI brain at baseline evaluation. 3 of $10(30 \%)$ patients deteriorated neurologically with in follow up period. Factors like MELD score and INR are significantly abnormal $(p=0.032$ and 0.01 respectively) in those patients who deteriorated neurologically started on chelation therapy.

Conclusions: One third patients of Wilson's disease who presented with hepatic or combined hepatic with neurological manifestations deteriorate on d-penicillamine therapy. Factors like MELD score and INR at the time of diagnosis are predictive factors for neurological deterioration in patients started on chelation therapy.

\section{PG3}

\section{Anorectal manometry for evaluation of chronic constipation in children}

Piyush Omprakash Somani, R T Narendran, Srinivasan Nisthala, Amit Gharat, Neeraj Sawalakha, Pravin Rathi.

Department of Gastroenterology, T N Medical College and BYL Nair Hospital, Mumbai 400 008, India

Purpose: The aim of this paper was to assess the clinical value of anorectal manometry (ARMM) for the evaluation of chronic constipation in children till 3 years of age.

Methods: From June 2008 to June 2010, 20 patients chronic constipation were analyzed. ARMM was performed using a desk, high rate gastrointestinal dynamic detection system.

Results: Out of twenty patients studied, the mean age was 10.66 months. 13 of them were male and 7 were females. The mean of average anal sphincter pressure was found to be $67.3 \mathrm{~mm}$ of hg. 15 patients had absence of inhibitory reflex of the anus (RIA) suggestive of Hirschsprung's disease. Later, on rectal biopsy, 10 out of 15 patients were found to have Hirschsprung's disease. Rest 5 patients were found to be normal.

Conclusions: ARMM is a simple, safe, and non-invasive method for evaluation of chronic constipation in children. 


\section{PG4}

\section{Enteroaggregative Escherichia coli interactions with intestinal mucosa}

\section{Deepika, ${ }^{1}$ B $R$ Thapa, ${ }^{2}$ Anuradha Chakraborti. ${ }^{1}$}

${ }^{1}$ Department of Experimental Medicine and Biotechnology,

${ }^{2}$ Department of Gastroenterology, Postgraduate Institute of Medical Education and Research, Chandigarh 160 012, India

Background: Enteroaggregative Escherichia coli (EAEC) has emerged as an important pathogen causing diarrheal disease in multiple epidemiological and clinical settings, yet its pathogenic mechanism is unclear. In here, attempts were made to study the interactions of EAEC with the host mucosal tissue in an In vitro organ culture (IVOC) model system.

Methods: EAEC isolates were collected from diarrhea patients (children $<5$ years), further screened and characterized for different aggregative adherence fimbrial (AAF) adhesins. Mucosal biopsies $(2-3 \mathrm{~mm} 2)$ were obtained from paediatric patients visiting PGIMER, Chandigarh, with ethical approval and parental consent during routine investigation of potential intestinal disorders by videoendoscopy (Fujinon EG/EC-41 paediatric endoscope). The mucosal biopsies were viable till $48 \mathrm{~h}$ of culture in DMEM and NCTC-135 medium (1:1) as determined by Trypan blue staining.

Results: The biopsies cultured to $24 \mathrm{hrs}$ infected with EAEC harboring different adhesins showed increased production of IL-8 after $6 \mathrm{hrs}$ as compared to other diarrheagenic E. coli. EAEC harboring AAF II adhesin produced more IL-8 at transcript and protein levels in comparison to other EAEC isolates.

Conclusion: This preliminary study involving duodenal biopsy sheds light on host-pathogen interaction in EAEC pathogenesis. Further studies are underway to reveal its interaction with the other regions of human intestine.

\section{PG5}

\section{Correlation of C/T-13910 variant with intestinal disaccharidase activities in different age groups of children among Indian population}

\section{Raja Amir, M Anwar, S Lal, B R Thapa, S Mahmood.} Department of Experimental Medicine and Biotechnology and Department of Gastroenterology, Postgraduate Institute of Medical Education and Research, Chandigarh 160 012, India

Aim: To correlate the C/T-13910 variant, associated with Lactase Persistence/Non-Persistence trait, with intestinal disaccharidase activities in children of different age groups among Indian Population.
Methods: Intestinal biopsies were obtained from 150 children aged 1 to 16 years undergoing upper gastrointestinal endoscopy because of various abdominal complaints. The biopsies were assayed for lactase and sucrase activities and genotyped for the C/T-13910 variant using PCR-RFLP and later on sequenced.

Results: There was a significant correlation between lactase activity and the $\mathrm{C} / \mathrm{T}-13910$ variant. The mean level of lactase activity among subjects with $\mathrm{C} / \mathrm{C}-13910$ genotype was about 3-6 U/g, and with C/T-13910 genotype was $20-29 \mathrm{U} / \mathrm{g}$.

Conclusion: Our study demonstrates a statistically significant correlation between the C/T-13910 genotype and intestinal lactase activity. Thus, C/T-13910 Single Nucleotide Polymorphism can be used as a potential biomarker for Lactase Persistence/Non-Persistence trait in Indian Population.

\section{PG6}

\section{Small bowel Crohn's disease vs. tuberculosis-A diagnostic dilemma: Barium enteroclysis has an answer}

Birinder Nagi, Manphool Singhal, ${ }^{*}$ Anupam Lal, ${ }^{*}$ Rakesh Kochhar, Deepak Kumar Bhasin, Surinder Rana, Niranjan Khandelwal, Kartar Singh.

Departments of Gastroenterology and Radio diagnosis, Postgraduate Institute of Medical Education and Research, Chandigarh 160 012, India

Objectives: To review the barium enteroclysis findings in proven cases of small bowel Crohn's disease (CD) and tuberculosis (ITB) for the purpose of diagnosing and differentiating these two diseases.

Methods: Retrospective analysis of radiological manifestations on barium enteroclysis over last 15 years in proven patients of small bowel Crohn's disease and tuberculosis was done. The radiological findings of both diseases were evaluated and compared.

Results: In CD, ileum was the commonest site affected in all the patients with conspicuous sparing of ileocecal junction, whereas in ITB ileocecal junction was the commonest site affected in $70 \%$ of cases. The classical radiological features of Crohn's disease on barium enteroclysis were ulceronodular pattern $(72 \%)$, eccentric strictures $(21 \%)$, asymmetric involvement with stretching of mesenteric border and pseudo-sacculation of antimesenteric border (15\%), perforations $(12.5 \%)$ and fistulae $(7.5 \%)$. In tuberculosis ileocecal junction was the commonest site affected with deformed ileocecal junction. Strictures were short, annular and concentric in tuberculosis seen in $62.3 \%$. Strictures were solitary in $61 \%$ while multiple in $39 \%$ of cases. Enteroliths were 
seen in $6.7 \%$ of patients in ITB. Separation of bowel loops was common in Crohn's disease as compared to matted bowel loops in tuberculosis.

Conclusions: Crohn's disease is a great simulator of tuberculosis; however barium enteroclysis which remains the cheapest modality that can diagnose and differentiate Crohns disease from tuberculosis.

\section{Nutrition}

\section{N1}

Chilled nasogastric insertion is more comfortable to patients than room temperature nasogastric tube

R Kaur, U Dutta, M T Noor, S Sharma, D Pankaj, R Kochhar, KSingh.

Department of Gastroenterology, Postgraduate Institute of Medical Education and Research, Chandigarh 16 012, India

Background: Nasogastric tube insertion is the most routine procedure in emergency department. Nasogastric tube placement can be highly painful for the patient. Cold therapy lowers metabolism, reduce vascular permeability to reduce conduction velocity of peripheral nerves. The aim of the present study to compare the comfort level of patient who received chilled nasogastric tube with those who received nasogastric tube kept at room temperature.

Methods: A randomized controlled trial was conducted involving 82 patients requiring nasogastric tube insertion. Study group $(n=41)$ received chilled nasogastric tube Control group $(n=41)$ received nasogastric tube kept at temperature while study group received nasogastric tube kept at 14 to $160 \mathrm{C}$. Assessment of pain level in the study and control group was done using Wong-baker faces pain rating scale (rated from 0 to 5).

Results: Mean age of cases and controls was $43.41 \pm$ 15.25 and $44.2 \pm 14.67$ years respectively $(\mathrm{p}=\mathrm{NS})$. In both the groups there were $24(65.85 \%)$ males and 14 $(34.14 \%)$ females $(p=N S)$. According to Wong-baker faces pain rating scale mean rank score of pain in cases was $2.88 \pm 1.20$ and in control group $3.63 \pm 1.0 \quad(p=$ 0.004 ), so there was significantly less pain in the group who received chilled nasogastric tube. Feeling of cooling sensation during tube insertion was more common in the study group than controls [39 (95.1\%) vs $12(29.2 \%)$, $p<0.01)$.

Conclusion: Chilled nasogastric tube insertion is associated with less patient discomfort that room temperature nasogastric tube insertion. We advocate the use of chilled nasogastric tube to reduce patient discomfort.
N2

\section{Obesity is associated with high serum leptin levels and low ghrelin levels}

Prasad R Anoop, M Sakpal, U Dutta, C K Nain, S K Sinha, $M T$ Noor, $R$ Kochhar, K Singh.

Department of Gastroenterology, Postgraduate Institute of Medical Education and Research, Chandigarh 160 012, India

Background: Leptin and Ghrelin are hormones which play a key role in regulation of energy expenditure and food intake. The role and interrelationship of these hormones to obesity in Indian subjects is unknown.

Aim: To determine the levels of serum leptin and ghrelin in obese and non obese controls and to study the interrelationship of these hormones with each other.

Methods: Cases were defined as obese individuals with BMI $23 \mathrm{~kg} / \mathrm{m}^{2}$ and controls (BMI-18.5 $22.9 \mathrm{~kg} / \mathrm{m}^{2}$ ). All were evaluated for anthropometry, liver and renal function test, lipid profile, fasting blood sugar, serum leptin and ghrelin levels. Normal levels of leptin were taken as 2 $11 \mathrm{ng} / \mathrm{ml}$, while for ghrelin as 300-800 pg/ml.

Results: The mean BMI of cases $(n=101$; females $71 \%)$ was higher than controls $(n=50$; females $78 \%)(32.1 \pm 6.9$ vs $20.8 \pm$ $\left.1.3 \mathrm{~kg} / \mathrm{m}^{2} ; p<0.001\right)$. Serum leptin levels were higher $(34.7 \pm$ 27.5 vs. $16 \pm 18.8 \mathrm{ng} / \mathrm{ml} ; p<0.001)$ and serum Ghrelin levels were lower $(105.6 \pm 134.7$ vs. $266.8 \pm 187 \mathrm{pg} / \mathrm{ml} ; p<0.001)$ in cases compared to controls. Leptin levels showed significant positive correlation with BMI (rho $=0.45, p<0.001)$, hip circumference $(r=0.52, p<0.001)$, waist circumference $(r=0.4$, $p<0.001)$. Serum ghrelin levels negatively correlated with BMI $(r=-0.29, p<0.001)$, hip circumference $(r=-0.46, p<0.001)$ and waist circumference $(r=-0.45, p<0.001)$. Serum leptin levels negatively correlated with serum ghrelin $(r=-0.35, p=0.012)$. Conclusions: High serum leptin and low ghrelin is found in majority of obese individuals. Leptin levels show a positive correlation with the surrogate markers of obesity and an inverse correlation with ghrelin levels.

\section{N3}

\section{Cellular deficiency of vitamin $B_{12}$ in patients with borderline serum vitamin $B_{12}$ levels}

R S Arun, E G Simon, A K Datta, A Chacko.

Department of GI Sciences, Christian Medical College, Vellore 632002, India

Background: Serum vitamin $\mathrm{B}_{12}$ estimation alone may not truly reflect vitamin $\mathrm{B}_{12}$ deficiency at cellular level in the borderline (low normal) range, on account of high intraindividual variation of test results. Homocysteine is a 
metabolic intermediate which is elevated in more than $95 \%$ of cases with vitamin B12 deficiency at cellular level.

Aim: To determine whether patients with borderline serum vitamin $\mathrm{B} 12$ levels have vitamin $\mathrm{B}_{12}$ deficiency at cellular level, using serum homocysteine as a surrogate marker.

Methods: The patients attending Gastroenterogy department at CMC Hospital, Vellore, and found to have serum vitamin $\mathrm{B}_{12}$ levels in the range of 200-400 pg/ml (normal 13micromoles). However, the serum homocysteine levels did not show a significant negative correlation with serum vitamin B12 levels (Pearson's correlation coefficient, $\mathrm{r}=-0.13 ; p=0.39$ ).

Conclusion: The majority (about 3/4th) of patients with borderline serum vitaminB12 levels have evidence of vitamin B12 deficiency at a cellular level, in the form of elevated serum homocysteine levels.

\section{Miscellaneous}

\section{MI1}

\section{Study of etiology of ascites at a tertiary referral centre}

Rajiv Baijal, Praveenkumar HR, Mayank Jain, Deepak Gupta. Department of Gastroenterology, Jagjivanram Western Railway Hospital, Mumbai

Aim: To determine the etiological factors responsible for ascites in patients presenting at our centre.

Methods: All patients admitted with ascites in gastroenterology ward between January 2008-January 2010 were included in the study.

Patients: History and symptoms were noted and relevant investigations were performed pertaining to individual case. Investigation performed were Ascitic fluid analysis, RFT, ADA level, Radiological investigation USG, CT scan (where necessary), 2DECHO (where necessary), and relevant tumor markers.

Results: In our analysis of 105 patients, the cause of ascites were cirrhosis (83) (alcoholic, hep B/C, cryptogenic, NASH), tuberculosis (5), renal disease (3), malignancy (5), pancreatic ascites (4), acute Budd-Chiari (3) and cardiac disease (2).

Conclusion: The most common etiological factor for ascites in our hospital is cirrhosis of liver (79\%).

\section{MI2}

\section{Experience with BIB balloon at our centre}

Rajiv Baijal, H R Praveenkumar, Mayank Jain, K Nagaraj, Deepak Gupta.

Department of Gastroenterology, Jagjivanram Western Railway Hospital, Mumbai
Background: Intragastric balloon is a non surgical, non pharmacological approach to weight loss. It is a soft, silicone balloon that is inserted in to the stomach and filled with sterile methylene blue saline. The presence of balloon creates the feeling of satiety or lack of hunger.

Results: A total of four cases underwent BIB insertion (two males and two females). The mean weight and BMI prior to insertion of balloon were $94.8 \mathrm{kgs}$ and $34.12 \mathrm{~kg} / \mathrm{m}^{2}$. After BIB insertion for 6 months, the average weight loss was $12.2 \mathrm{~kg}$. The common problems experienced with the balloon were vomiting, retching and bloating sensation in the initial few days. None of the patients had migration or rupture of the balloon.

Conclusion: BIB is a safe method for weight loss. However, the role of diet and exercise is paramount. Strict follow up with emphasis on diet and exercise is mandatory in patients with BIB.

\section{MI3}

\section{Association between functional polymorphisms in serotonin reuptake transporter -P gene and microscopic colitis in North Indians}

S V Rana, A Sikander, K K Prasad, R P Ola, S K Arora, $S$ K Sinha, K Singh.

Department of Gastroenterology, ${ }^{*}$ Department of Immunopathology, Postgraduate Institute of Medical Education and Research, Chandigarh 160 012, India

Background: Serotonin (5-hydroxtryptamine, 5-HT) is an important factor in gut function, playing key role in intestinal peristalsis, secretion, and in sensory signaling in the brain-gut axis. Removal from its sites of action is mediated by a specific protein called serotonin reuptake transporter (SERT or 5-HTT). Polymorphisms in the promoter region of SERT gene have effects on transcriptional activity, resulting in altered 5-HT reuptake efficiency. It has been speculated that such functional polymorphisms may underlie disturbance in gut function in individuals suffering from disorders such as Microscopic Colitis (MC). Aim: The aim of this study was to assess the potential association between SERT-P gene polymorphisms and microscopic colitis in North Indians.

Method: This prospective case-control study included 82 MC patients and 100 healthy controls. SERT-P gene polymorphisms were studied by polymerase chain reaction. Result: The significant difference was observed in SERT-P genotype $(\mathrm{X} 2=6.0, P<0.05)$ and allele frequencies $(p<0.05)$ between the MC patients and controls. The frequencies of Non-S genotype were significantly higher than S/S genotype between the $\mathrm{MC}$ patients and controls $\{\mathrm{OR}=3.1(\mathrm{CI}=1.1-$ 8.63); $P<0.05\}$. The frequencies of genotype were similar in both males and females in patients and controls. 
Conclusions: A significant association was observed between SS genotype of serotonin reuptake transporter-P polymorphism and microscopic colitis in North Indians.

\section{MI4}

\section{Does febrile illness precipitate upper GI bleed?}

Krishnadas Devadas, $R$ Sobhana Devi.

Department of Gastroenterology, Medical College, Kottayam

Introduction: Kerala is in the grip of recurrent epidemics of viral fevers. Patients develop thrombocytopenia with viral fevers and usually are given antipyretics and analgesics. There always is a fear of precipitating GI bleeds.

Methods: The hospital and endoscopy records of three consecutive years (from July 2007 to June 2010) were retrospectively analysed to look for any correlation between admissions for fever and bleeds. The different age groups were also analysed. Statistical analysis was done using SPSS software version 18 .

Results: There were a total of 8653 admissions for fever including 2030 for viral fevers. There were a total of 1744 bleeds. Of these, $728(42 \%)$ were due to portal hypertension (esophageal varices $524(30 \%)$, gastric varices 27 , portal hypertensive gastropathy 177). There were 230 ulcer bleeds (13.2\%) (141 duodenal ulcers (DU), 80 gastric ulcers (GU) and 9 combined DU and GU). There were 168 cases of erosions in stomach, 213 Mallory Weiss tears and 71 esophagitis. 165 endoscopies were normal. Significant correlation was found only between GU bleeds and viral fever $(p=0.006$, correlation coefficient 0.474 ) when all age groups were considered together. This correlation was lost when the age groups were differentiated into middle age (36-65 yrs) and aged ( $>65 \mathrm{yrs})$. There was no correlation between all bleeds, bleeds due to portal hypertension or DU with all fevers or viral fevers. Conclusions: Majority of bleeds are due to portal hypertension. GU bleeds are increased during epidemics of fever probably due to inappropriate use of drugs. Febrile illness does not seem to increase the chance of bleed in the aged population.

\section{MI5}

Analysis of alcohol content, $\mathrm{pH}$ and acidity of alcoholic preparations as consumed by people in community

\section{T Noor, K Singh, C K Nain, U Dutta, K K Prasad.} Department of Gastroenterology, Postgraduate Institute of Medical Education and Research, Chandigarh 160 012, India

Background: The magnitude of the problem related to alcohol use and the extent of health consequences of alcohol consumption are alarming in most countries of the
South-East Asia region. Adverse health effects of alcoholic liquor are directly proportional to its ethanol content. Alcohol content of different alcohol preparations is variable. We studied $\mathrm{pH}$, acidity and alcohol content of different alcohol preparations as available and consumed by the people in community at large.

Methods: Different sites were visited in Chandigarh, Punjab and Haryana states to collect alcohol samples. $\mathrm{pH}$ was determined using micropH glass electrode, acidity was measured by titration method using phenol red as an indicator. Alcohol content was noted as stated on the labels of the containers.

Results: A total of 242 samples were analyzed. Thirteen samples were 600 proof, their $\mathrm{pH}$ was $5.32 \pm 0.60$ and acidity was $0.63 \pm 0.27 \mathrm{meq} / \mathrm{L}$. Eighty two samples were 500 proof, their $\mathrm{pH}$ was $5.15 \pm 0.73$ and acidity was $0.67 \pm 0.48 \mathrm{meq} / \mathrm{L}$. Eight six samples were 750 proof, their $\mathrm{pH}$ was $4.90 \pm 0.81$ and acidity was $3.80 \pm 10.04 \mathrm{meq} / \mathrm{L}$. There was one 800 proof sample and one 65.60 proof sample each. Alcohol content of 59 samples was not known, $\mathrm{pH}$ of these samples was $5.5 \pm$ 1.17 and acidity was $1.17 \pm 2.30 \mathrm{meq} / \mathrm{L}$. Nine (3.7\%) samples had strong acidity $(\mathrm{pH}<4), 34$ samples were alkaline $(\mathrm{pH}>7)$. Conclusion: Alcohol content, $\mathrm{pH}$ and acidity of the alcoholic preparations consumed by the people in community is highly variable. Most of the preparations are acidic although alkaline $\mathrm{pH}$ is also not uncommon.

\section{MI6}

\section{Assemblage study of Giardia intestinalis isolates in North Indian population}

Vibhor Tak, Pooja Yadav, B R Mirdha, Govind Makharia, Shinjini Bhatnagar.

Departments of Microbiology; Gastroenterology and Human Nutrition Unit, All India Institute of Medical Sciences, New Delhi 110 029, India

Background: Giardia intestinalis, a common intestinal pathogenic protozoan parasite causes infections that range from asymptomatic cyst passage to symptomatic diarrhea (acute, persistent or chronic), weight loss, and malabsorption. Genetic characterization of Giardia intestinalis isolates has revealed the existence of seven groups (assemblages $A$ to $G$ ) in different host species. However, only assemblages A and B cause infections in humans.

Methods: 82 patients positive for Giardia intestinalis by light microscopy were enrolled for the present study. DNA was extracted for all these isolates. Molecular characterization by polymerase chain reaction (PCR) was carried out using Giardia specific triose phosphate isomerase (tpi) gene and restriction fragment length polymorphism (RFLP) assay was performed using Rsal restriction enzyme. The sensitivity of the tpi gene PCR assay for the detection of Giardia 
intestinalis was determined by making 10 fold serial dilutions (10-1 to 10-10) of the extracted DNA.

Results: 82 patients including 40 adults and 42 children presented with acute (8), persistent (1) or chronic (61) diarrhea. 12 patients had no diarrheal complaints. All the isolates were identified as Assemblage B irrespective of the clinical presentation of the disease. The analytical sensitivity of the isolates with occasional cyst and $0-2 /$ hpf cyst load was corresponding to 10-5 and 10-6 dilutions respectively, in the PCR assay.

Conclusions: The predominance of Assemblage B indicates probably the anthroponotic transmission in the study population. Further studies with larger number of clinical and environmental samples would be able to extrapolate the true diversity and transmission dynamics of this protozoan in our population.

\section{MI7}

\section{An unusual cause of abdominal distension: The gastrointestinal stromal tumor}

Ajit Kumar, Yogesh Harwani, Akash Chaudhary, Nayana Joshi, A.C. Upadhyaya.

Department of Gastroenterology, Nizam's Institute of Medical Sciences, Panjagutta, Hyderabad 500 082, AP

Introduction: GIST presenting as diffuse abdominal distension is rare. We report such a case where the reason for admission was evaluation of diffuse abdominal distension which included tense ascites versus psuedomyxoma peritoneii etc.

Case Report: A 40 yr old male presented with abdominal distension and low grade daily intermittent fever for 3 months. Examination revealed abdominal distension, dull note all over abdomen, no mass. He had anemia (Hb- $9.8 \mathrm{~g} / \mathrm{dl}$ ) and hypoalbunemia $(2.2 \mathrm{~g} \%$ ) rest biochemical profile and hemogram was unremarkable. USG demonstrated heterogeneous lobulated hypo echoic lesion occupying whole of the abdomen, extending from the level of liver upto the bladder level, increased vascularity on Doppler, with DD? carcinomatosis v/s. psuedomxyoma peritoneii. Tumour markers CEA, AFP, CA 19-9 were normal. UGI scopy and colonoscopy were normal. CT abdomen revealed large heterogeneously enhancing lobulated mass occupying whole peritoneal cavity with bowel loops displaced medially, few cystic areas seen with liver scalloping suggestive of peritoneal carcinomatosis v/s. psuedomxyoma peritonei. Fluid analysis: hemorragic, no leucocytes, glucose $31 \mathrm{~g} / \mathrm{dl}$, proteins $3.7 \mathrm{~g} / \mathrm{dl}$, albumin $1.0 \mathrm{~g} / \mathrm{dl}$. Cytology no malignant cells. Biopsy showed spindle cells arranged in small fascicles, moderate cytoplasm with elongated nucleus and bland chromatin, no areas of necrosis and mitosis seen. IHC revealed CD 117 positive s/o GIST ? extraintestinal (mesenteric/omental).

Diagnosis: Extra intestinal GIST: mesenteric/omentum or luminal GIST with exophytic growth. Surgical opinion opined as inoperable tumour. Pt started with imitanib $400 \mathrm{mg}$ BD.
Conclusion: GIST is a rare cause of diffuse abdominal distention which is often not considered in the differential diagnosis.

\section{MI8}

Retrospective analysis of endoscopic findings in patients with UGI bleeds-NIMS Experience

Akash Chaudhary, Ajit Kumar, J Sandeep Reddy, A C Upadhayaya, Nayana Joshi, K Manoj, K Vishnu, C Padmavathi, Yogesh Harwani.

Department Of Gastroenterology, Nizam's Institute of Medical Sciences, Hyderabad 500 082, AP

Background: Upper GI bleed (UGI B) forms a major indication for endoscopic evaluation in current clinical practice. Esophago-gastric varices and peptic ulcers are the most common cause for upper GI bleed.

Aim: To study the endoscopic findings in patients evaluated for UGI bleed.

Method: Endoscopic findings in patients undergoing endoscopy for upper GI bleed from January 2008 to December 2009 were retrospectively analyzed from our database.

Result: A total of 159 patients underwent endoscopy for upper GI bleed. There were 131 males $(82.4 \%)$ and $28(17.6 \%)$ females. The age ranged from 16 years to 98 years with dominant involvement in 5th decade. Peptic ulcers were seen in 40 patients accounting for $(25.15 \%)$ in which duodenal ulcer were seen in 23 patients and Gastric ulcer in 17 patients. Bleeding esophageal varices were seen in 49 patients $(30.8 \%)$ and Gastric varices in 8 patients $(5 \%)$. Other findings were MWT in 8 patients (5\%), Diaulefoys lesion in 2 patients $(1.2 \%)$, gastro-duodenal malignancy in10 patients $(6.3 \%)$, hemosuccus pancreaticus in 3 patients $(1.9 \%)$, gastric polyps in 2 patients $(1.2 \%)$ corrosive injury in 2 patients $(1.2 \%)$, Erosion in 26 patients (16.3\%), esophagitis 17 patients (10.6\%). Endoscopy was normal in 19 patients (11.9\%).

Conclusion: Esophageal varices and Peptic ulcer disease was found to be the commonest endoscopic finding in patients presenting with UGI bleed.

\section{MI9}

CYP2C19 polymorphism as a predictor of personalized therapy in South Indian population

S Siva Prasad, ${ }^{1}$ Rupa Banerjee, ${ }^{2}$ Manu Tandon, ${ }^{2}$ N Prathap, ${ }^{2}$ M Sasikala, ${ }^{1}$ D Nageshwar Reddy. ${ }^{2}$

${ }^{1}$ Asian Health Care Foundation, ${ }^{2}$ Asian Institute of Gastroenterology, Somajiguda, Hyderabad 500 082, India

Introduction: The cytochrome P4502C (CYP2C) subfamily of enzymes metabolizes approximately $20 \%$ of drugs 
commonly used in clinical practice. Based on genotype of CYP2C19 and metabolic rate of various drugs, individuals are classified into homozygous extensive metabolizers (Hom EM), heterozygous extensive metabolizers (Het EM) and poor metabolizers (PM). Genetic polymorphism of CYP2C19 occurs with varying frequency (3-70\%) among different ethnic groups. Since there is lack of conclusive data on the genotyping of CYP2C19 (*2 and *3) polymorphism in South Indian population, the present study was aimed for the first time to determine the frequency of Poor metabolizers in South Indian population. Methods: A total of 220 healthy individuals were recruited in the study. Genotyping of CYP2C19*2 and *3 polymorphisms was performed by a Polymerase chain reactionRestriction fragment length polymorphism method.

Results: The genotypic results of both CYP2C19*2 and CYP2C19*3 were taken into consideration to establish the frequency of poor metabolisers. Individuals who were homozygous for $\mathrm{m} 1(\mathrm{~m} 1 / \mathrm{m} 1)$ and $\mathrm{m} 2(\mathrm{~m} 2 / \mathrm{m} 2)$ and heterozygous for both $\mathrm{m} 1$ and $\mathrm{m} 2(\mathrm{~m} 1 / \mathrm{m} 2)$ mutations were categorized as poor metabolizers. In this study one individual was heterozygous for $\mathrm{m} 1 / \mathrm{m} 2$ mutation and 32 participants were homozygous for $\mathrm{m} 1 / \mathrm{m} 1$ mutation. The frequencies of Hom EM, Het EM and PM for CYP2C19 genotypes were $71(32.2 \%), 116(52.8 \%)$ and $33(15 \%)$ respectively. The minor allele frequency of CYP2C19*2 and 3 was $0.41 \%$ and $0.1 \%$ respectively.

Conclusion: The study result can be the basis for the standardization of personal medicine in South Indian population.

\section{MI10}

Thiopurine methyltransferase as a therapeutic indicator of purine analogues: a study from Southern India

A M Sadik, ${ }^{1}$ S Siva Prasad, ${ }^{1}$ M Guru Prasad, ${ }^{1}$ M Sasikala, ${ }^{1}$ Manu Tandon, ${ }^{2}$ Rupa Benerjee. ${ }^{2}$

${ }^{1}$ Asian Health Care Foundation, ${ }^{2}$ Asian Institute of Gastroenterology, Somajiguda, Hyderabad 500 082, Andhra Pradesh, India

Introduction: The purine analogues used to treat certain chronic diseases are occasionally associated with bone marrow suppression and leucopenia. They are metabolized by thiopurine S-methyltransferase (TPMT) to methyl mercaptopurines. Thus optimal TPMT activity of an individual plays an important role in the treatment. However a wide interindividual variation of TPMT activity may be multi-factorial including inter and intra familial genotypic variation of TPMT gene. The present study was aimed to establish the reference range of TPMT activity and its relevance with genotype.
Methods: 165 healthy blood donors were assessed for their TPMT activity by HPLC-UV method and 150 of them were analyzed for TPMT $* 2, * 3 \mathrm{~A}$ and $* 3 \mathrm{C}$ polymorphisms by PCR-direct sequencing.

Results: The mean age of study cohort was $28 \pm 9.8$ (19-58) years. The range of TPMT activity was found to be wide (3.93 to $35.80 \mathrm{nmolml}-1 \mathrm{~h}-1$ PRBC). The mean of the TPMT activity in total population was $16.51 \pm 7.66$, in females $10.99 \pm 3.44$ and in males it is $17.83 \pm 7.81$. All the participants studied for TPMT gene polymorphism were found to be wild type for all major polymorphic regions. Conclusion: The range of TPMT activity is wide by virtually. The wide reference range of enzyme activity may have multi-factorial basis. The frequency of hetero and homozygous variants of screened polymorphisms in TPMT gene may be less frequent in South Indian population.

\section{MI11}

\section{Endoscopic findings during pre renal transplant evalution in end stage renal disease patients}

\section{S Raja, K Arunkumar, V Jayanthi.}

Department of Gastroenterology, Stanley Medical College Hospital, Chennai 600 003, India

Background: Patients with end-stage renal disease (ESRD) may demonstrate a number of gastrointestinal lesions and suffer subsequent complications. Gastrointestinal complications include esophagitis, peptic ulcer, diarrhea, colon disorders. Duodenitis and mucosal erosions are commonly seen in patients with chronic kidney disease (CKD). Aim: To study the endoscopic findings in ESRD patients. Methods: We studied consecutive patients with end-stage renal disease who underwent upper gastrointestinal routine endoscopy before renal transplantation during August 2008 and July 2010 were collected and 286 patients were analyzed. Results: We studied 287 patients 216 (75.5\%) male, 70 (24.5\%) female. Mean age was 38.5 . Of the patients $73.8 \%$ were asymptomatic but some experienced nausea (12.6\%), heartburn (8.7\%), and abdominal pain (7.3\%). Endoscopy was normal in 137 patients (47.7\%). Abnormal endoscopic findings were duodenal erosions in15 (10.1\%) patients, antral erosions in $26(17.4 \%)$ patients, gastritis in 28 $(18.8 \%)$ patients, duodenal ulcer in $15(10.1 \%)$ patients, esophagitis in $32(21.5 \%)$ patients, Moniliasis in $5(3.3 \%)$ patients, duodenal ulcer in $20(13.4 \%)$ patients, nodular duodenum in $1(0.6 \%)$ patients, and inflammatory gastric polyps in $2(1.3 \%)$ patients, periampullary diverticulum 2 $(1.3 \%)$ patients, growth stomach in $1(0.6 \%)$ patient esophgeal varices in $2(1.3 \%)$ patients.

Conclusions: Duodenal erosions (10.1\%), gastric erosions (17.4\%), and duodenal ulcer (10.1\%), esophagitis (21.5\%) 
are common lesions in patients with end-stage renal disease. Male genders are higher risk of these lesions. As there is no association between patients symptoms and gastro duodenal lesions, which may increase the risk of post-transplant complications, the development of diagnostic strategies for the detection of these lesions is recommended.

\section{MI12}

\section{Incidence of methanogenic flora in apparently healthy subjects from different regions of North India}

S V Rana, J Kaur, R P Ola, S Sharma, V P Singh, K Singh. Department of Gastroenterology, Postgraduate Institute of Medical Education and Research, 160 012, India

Introduction: With the presence of methanogenic flora, 4 mol of hydrogen $(\mathrm{H} 2)$ are used with 1 mol of carbon dioxide $(\mathrm{CO} 2)$ to produce $1 \mathrm{~mol}$ of methane $(\mathrm{CH} 4)$, a process greatly reducing the volume of gas in the colon thus reducing flatulence. The incidence of methanogenic flora may be different in different regions of North India.

Aim: Therefore, this study was planned to measure methane concentration produced by methanogenic flora in apparently healthy subjects from Himachal and Punjab regions of North India, by using lactulose breath test.

Methods: This study was conducted in 150 apparently healthy adult subjects of age range $25-58$ years. Out of these 85 were from Punjab region and 65 from Himachal. Methane concentration was measured (by using SC Microlyser from Quintron, USA) in end expiratory breath at fasting and after every $30 \mathrm{~min}$ for next $4 \mathrm{hrs}$ after giving lactulose. Results: There were 57 males (67\%) from Punjab and 49 $(75 \%)$ males from Himachal. Age (Mean \pm SD) of subjects from Punjab was $41.6 \pm 16.5$ years while in subjects from Himachal $43.7 \pm 14.3$ years. Methane production was in 12 / 85 (14\%) subjects from Punjab and 28/65 (43\%) subjects from Himachal.

Conclusion: This study indicates that methanogenic flora was significantly higher in subjects from Himachal than from Punjab region.

\section{MI13}

\section{Aetiology of upper GI bleeds in a tertiary care centre in South India}

Krishnadas Devadas, Sobhana Devi. Medical College, Kottayam

Introduction: Literature on aetiology of upper gastrointestinal (UGI) bleeds in adults in India is scarce. Contrary to the West, common causes like ulcer bleeds often take a back seat in our population.
Methods: The endoscopy records of the Department of Gastroenterology, Medical College, Kottayam over the past five years (2005 July to 2010 June) were retrospectively perused to look for the cause of bleed in those patients who presented with hematemesis and / or malena. The causes of UGI bleed overall and in the age groups - below 36 yrs, 36$65 \mathrm{yrs}$ and above $65 \mathrm{yrs}$ were analysed. Statistical analysis was done using the Chi Square test.

Results: There were a total of 2524 UGI bleeds with male to female ratio of 3.4:1 (1.4:1 in the above $65 \mathrm{yr}$ and 5:1 in the 36$65 \mathrm{yr}$ group). $65.4 \%$ of patients were $36-65 \mathrm{yrs}$ of age. Bleeds due to Portal Hypertension (PHT) (esophageal varix (28.8\%), Gastric varix (1.67\%), portal hypertensive gastropathy $(9.35 \%))$ were most common $(39.82 \%)$ followed by ulcer bleeds (15.97\%). Ulcer bleeds were more common than PHT bleeds in patients above 65 yrs $(P<0.0001)$ in both the sexes. $40.43 \%$ of all ulcer bleeds occurred in this age group. There were significantly more DU bleeds than GU bleeds across all age groups. $9.5 \%$ of endoscopies were normal with females significantly more likely to have a normal endoscopy (OR 3.75, CI 2.85-4.94, $P<0.0001$ ). $11.93 \%$ had Mallory Weiss tear, $10.18 \%$ erosive gastropathy and $3.5 \%$ had esophagitis. Conclusions: Bleeds due to portal hypertension are more common in our population compared to ulcer bleeds. However ulcer bleeds are more common in the elderly. Up to $10 \%$ of endoscopies following an UGI bleed may be normally, especially in females.

\section{MI14}

A single center experience of EUS guided fine needle aspiration cytology (FNAC) in various lesions

A Bapaye, ${ }^{1}$ A Aher, ${ }^{1}$ V Bhide, ${ }^{2}$ V Joshi, ${ }^{2}$ H Gadhikar, ${ }^{1}$ L Shimpi, ${ }^{1}$ ${ }^{1}$ Department of Digestive Diseases and Endoscopy, ${ }^{2}$ Department of Pathology, Deenanath Mangeshkar Hospital and Research Center, Pune, India

Introduction: Over a 4 year period (2006-10), 160 patients underwent EUS guided FNAC / FNAB for mass lesions in and around the upper gastrointestinal tract.

Methods: EUS was performed using Fujinon SU7000 EUS system and EG530UT linear echoendoscope (Fujifilm Inc., Japan). 180 lesions were sampled in 160 patients. After locating the lesion by EUS, FNA was obtained using a 22G Echotip-Ultra needle (Cook, USA). Onsite pathologist was available for 73 lesions in 61 patients, 38\% (Group 2) whereas absent in 99 patients 107 lesions, 61\% (Group 1).

Results: Overall accuracy in our series was $89 \%$. Organs sampled were pancreas $92(51 \%)$, lymph nodes $50(27 \%)$, duodenum $11(6 \%)$, esophagus $6(3 \%)$, stomach $4(2 \%)$, gall bladder $4(2 \%)$, liver $4(2 \%)$, CBD $3(2 \%)$, mediastinal 
and retroperitoneal tumors $3(2 \%)$ each. Cytopathology revealed malignant / metastatic lesions in 61 (34\%), benign $17(9 \%)$, inflammatory 47 (26\%), inconclusive 20 (11\%). Adequate samples not representative of pathology were obtained in 35 (19\%). Inconclusive reports were significantly reduced in group $2 \mathrm{v} / \mathrm{s}$ group 1-3/61 (5\%) v/s 17/107 $(15 \%), p<0.05$. Positivity of samples improved when FNAC was combined with FNAB (clot in formalin) $8 / 130$ $(6 \%) \mathrm{v} / \mathrm{s}$ FNAC alone 12/50 (24\%), $p<0.005$. 64/125 (51\%) patients could be treated conservatively due to a confirmed benign / inflammatory pathology.

Conclusions: Presence of onsite pathologist significantly increased EUS-FNA yield. FNAC combined with FNAB significantly improved diagnostic yield. EUS-FNA avoided surgery in half the patients.

\section{MI15}

\section{Cutaneous metastases-An uncommon presentation of intra-abdominal visceral malignancy}

Vivek Srivastava, Vaibhav Pandey, Anand Kumar. Banaras Hindu University, Varanasi 221 005, Uttar Pradesh

Background: Cutaneous metastases occur infrequently in intra-abdominal malignancies and rarely present at initially diagnosis. We report two cases, one patient of laparoscopic cholecystectomy developing port site metastasis in incidental gallbladder cancer and second case of bilateral renal cell carcinoma with multiple cutaneous secondries.

Case I: A middle aged female underwent laparoscopic cholecystectomy and was diagnosed as incidental gallbladder cancer (papillary adenocarcinoma) with T1a stage. She was advised for 3 monthly follow up to which she did not comply. Nine months post-operatively she again presented with a huge epigastric-port mass which on FNAC showed deposits of adenocarcinoma. A CECT thorax and abdomen was done which showed a huge parietal mass without any recurrence or metastasis. A wide local excision of full anterior abdominal wall with mesh repair and local transposition flap reconstruction followed by EBRT was done. The patient is doing well at 6 months of follow up.

Case II: A 70 year male presented with recent onset rapidly developing multiple cutaneous nodules. He was otherwise asymptomatic and systemic examination was normal. An excision biopsy was done and histopathology showed deposits from adenocarcinoma. An ultarasonographic evluation showed bilateral renal mass with infiltration in IVC and multiple retroperitoneal lymphadenopathy. Patient was not affordable and refused any further treatement.
Conclusion: Patients with an incidental gallbladder cancer after laparoscopic cholecystectomy can have port site recurrence even in T1a tumors hence the port excision should be done in such cases. Cutaneous metastasis can be a presenting symptom of otherwise asymptomatic renal cell carcinoma.

\section{MI16}

\section{Clinical spectrum of inflammatory bowel disease in a tertiary health care centre in India}

Rupa Banerjee, Magnus J Mansard, Nitesh Pratap, Mohan Ramchandani, Manu Tandan, G. V. Rao, D. Nageshwar Reddy. Asian Institute of Gastroenterology, Somajiguda, Hyderabad 500082 , India

Introduction: Inflammatory bowel disease (IBD), especially Crohn's disease (CD) was considered rare in India till recently. There are no large scale epidemiological data of inflammatory bowel disease (IBD) from the Indian subcontinent. Differences in the environmental and genetic back ground have altered the clinical epidemiology, diagnosis and treatment of IBD in the Asia Pacific population compared to the western world. A study of the patient characteristics selective to the subpopulation is an essential step for optimizing management accordingly.

Methods: All patients diagnosed to have IBD in our centre between December 2004 and August 2009 were prospectively evaluated using a specially designed questionnaire.

Results: IBD was diagnosed in 717 patients, 274 patients had CD and 443 patients had UC. Male predominance was noted in both groups (CD F:M = 1: 1.415 , UC F:M = 1:1.53). Mean age of onset was 31.82 years, no second peak in age wise incidence was found. CD patients were younger than UC patients (28.58 yrs vs. $33.69 \mathrm{yrs}, p<0.0001)$. The duration of symptoms for UC and CD were 21.43 months (SD26.658 months) and 21.92 months (SD- 23.281 months), respectively. Diarrhea (57.44\%), abdominal pain (58.44\%) and weight loss $(22.03 \%)$ were common symptoms. Extraintestinal manifestations included predominanatly arthralgia $(73.47 \%)$ with eye and skin manifestations found to be rare (in 49 and 6 patients respectively). Complications included portal vein thrombosis in 6 subjects, 1 patient had acute mesenteric thrombosis with jejunal gangrene. Optic neuritis was found in 1 patient, enterocutaneous fistula in 3 patients and pyoderma gangrenosum in 4 subjects. The site of involvement for UC patients: left sided colitis in 200 patients $(45.15 \%)$, pancolitis in 177 patients $(39.95 \%)$ and proctitis in 66 
patients $(14.90 \%)$. CD patients had predominantly colonic involvement (52.2\%), small bowel was involved in only $18.2 \%$. Perianal disease was found in 7 patients $(2.53 \%)$ and duodenal involvement was found in 4 patients $(1.46 \%)$. Family history of the disease was found in 61 patients $(8.5 \%)$. A single case of UC related malignancy was found in patients on follow up. Mean delay in diagnosis was 21.45 months (SD- 26.658) with an average of 2.53 (SD- 1.469) physicians were consulted before the correct diagnosis was made.

Conclusion: This the largest cohort of IBD patients reported from India. The epidemiology of IBD in India appears similar to reports from the Asia Pacific region where IBD is an emerging disease. The delay in diagnosis reflects the complex clinical picture and lack of awareness about IBD. A better understanding of the clinical profile would enable development of practical guidelines for IBD management in India.

\section{MI17}

\section{Tuberculosis presenting as isolated hepatic and splenic granulomas: a rare case report}

Ajit Kumar, C Padmavathy, Akash Chaudhary, A C Upadhyaya, Nayana Joshi, Manoj Kumar, Yogesh Harwani, Sandeep Reddy.

Department of Gastroenterology, Nizam's institute of Medical Sciences, Hyderabad 5-00 082, AP

Introduction: Isolated chronic tubercular granulomas of liver and spleen without involvement of bone marrow or other organs is a very rare presentation.

Case Report: A 55 year old with woman DM type II on OHA presented with H/o RUQ pain and Low grade, Irregular fever of 2 months duration. She was evaluated elsewhere, found to have Gall stones and Lap. cholecystectomy was attempted. During surgery multiple liver nodules were noted and cholecystectomy was deferred. She came to us for further evaluation. On examination she had moderate hepato-splenomegaly with irregular liver surface without peripheral lymphadenopathy or ascites. Other systemic examination was normal. On investigations hemogram, biochemical profile including LFT, PT and RFT was normal. AFP: $3.9 \mathrm{ng} / \mathrm{ml}$. Montoux test: negative. CXR: Normal. Calcium: $8 \mathrm{mg} / \mathrm{dl}$; S. ACE levels: $57 \mathrm{U} / \mathrm{L}$ (Normal). Hepatitis viral markers: negative; ANA Negative. CECT Abd: Non enhancing, multiple isodense lesions in liver and spleen. Bone marrow aspiration and biopsy was unremarkable. UGI endoscopy: Normal study. Liver biopsy: Periportal lymphomononuclear infiltrates and macrovesicular steatosis, focal cholestasis and bile ductular proliferation along with non caseating epitheloid granulomas with Langhans type and FB type giant cells and surrounding fibrosis of possible Koch's etiology noted. Fungal Stains were Negative. She was started on ATT- 4 drugs. A significant symptomatic improvement was noted after 6 weeks.

Conclusion: Isolated chronic tubercular granulomas of liver and spleen without involvement of bone marrow or other organs do occur though rarely. They often defy the diagnosis despite extensive work-up.

\section{MI18}

Efficacy of Drotaverine hydrochloride $80 \mathrm{mg}$ (Drotin DS) tablet for abdominal pain associated with irritable bowel syndrome (IBS), a double blind placebo controlled study

Manisha Dwivedi, ${ }^{1}$ S P Misra, ${ }^{1}$ Vatsala Misra, ${ }^{2}$ Sanjay Pant, ${ }^{1}$ Sushil Narang ${ }^{1}$ Ajay Kumar. ${ }^{3}$

${ }^{1}$ Departments of Gastroenterology, ${ }^{2}$ Pathology, M L N Medical College, Allahabad $211001,{ }^{3}$ Centre for Biotechnology, Allahabad University, Allahabad

Purpose: The aim of this study was to evaluate the efficacy of drotaverine hydrochloride $80 \mathrm{mg}$ tablets in relief of abdominal pain associated with irritable bowel syndrome (IBS).

Methods: Sixty adult patients, more than 18 years old having IBS according to Rome II criteria were enrolled in the study. Thirty subjects received drotaverine hydrochloride $80 \mathrm{mg}$ TDS for 28 days and remaining thirty received placebo randomly for the same period. Each subject was followed on a weekly basis to see for pain relief. Biochemical analysis such as serum alanine amino transferase, serum aspartate amino transferase, hemogram and occult blood in stool was done at the start of study.

Result: In the intervention group (Drotin DS) of the 30 patients $23(76.6 \%)$ had complete follow up of which $21 / 23$ (91.3\%) had complete relief from pain and no adverse effect. $2 / 23(8.7 \%)$ had no relief in pain but reported no adverse effect at the same time. In the placebo group 22/30 (73.3\%) came for complete follow up of which 11/22 $(50 \%)$ had complete relief in pain. Seven out of $22(31.8 \%)$ had mild to moderate relief.Two out of $22(9.1 \%)$ maintained status quo for the symptoms and 2/22 (9.1\%) had worsening of symptoms.

Conclusion: It was concluded that Drotin DS gives a better response than placebo in pain relief in patients with IBS. The improvement in the Drotin DS group was $91.3 \%$, while the improvement in the placebo group was $50 \%(p<0.05)$. 


\section{MI19}

T-Cell lymphoma presenting with ascites and raised CA-125-Report of a case

Archana Rastogi, Chaggan Bihari, Deepak Jain, Vikram Bhatia. Departments of Pathology, Radiology and Hepatology, ILBS, Delhi

Malignant lymphoma rarely present with ascites without a known history of malignancy. Due to such uncommon occurrence it receives much less attention than peritoneal carcinomatosis in clinical practice. This is a report of an unusual case of T- cell lymphoma presenting with ascites, omental thickening and raised serum glycoprotein-125 (CA125) and high ascitic fluid Adenosine deaminase (ADA) levels. This case was clinically suspected to be peritoneal carcinomatosis or peritoneal tuberculosis based on clinical, biochemical and radiological features. Effusion cytology, cell block and immunohistchemistry confirmed this case as T-cell lymphoma. Three days later, histologic examination of gastric biopsy revealed infiltration by atypical lymphoid cells with identical immunophenotypic profile. This case demonstrates the value of effusion cytology, cell block preparation and immunohistochemistry for the diagnosis of clinically unsuspected difficult case of lymphoma.

\section{MI20}

\section{Role of endoscopic ultrasound (EUS) in evaluation of idiopathic acute pancreatitis (IAP)}

\section{S S Rana, D K Bhasin, A Agarwal, K Singh.}

Department of Gastroenterology, Postgraduate Institute of Medical Education and Research, Sector 12, Chandigarh 160012 , India

Aims: Pancreatic cystic lesions not associated with pancreatitis are a diagnostic dilemma and EUS has helped in better evaluation of these lesions.

Methods: Retrospective analysis of all the patients with pancreatic cystic lesions not following an attack of pancreatitis referred to us for EUS was done.

Results: Over last 20 months, 9 patients (6 M; age: 1967 years) with pre EUS diagnosis based on cross sectional imaging of pseudocyst (5), cystic tumor (3) and IPMN (1) were evaluated. Of 5 patients with pseudocysts, EUS appearances were suggestive of pseudocyst (2), cystic tumor (2) and main duct IPMN (1). EUS FNA confirmed the diagnosis of cystic tumor in 2 patients (mucinous tumor (1) and pseudopapillary tumor (1) and main duct IPMN in 1 patient. However, 2 patients with appearances suggestive of pseudocyst on EUS had simple cyst (1) and mucinous tumor (1) diagnosis after FNA. Three patients with cystic tumor on cross sectional imaging had EUS appearances suggestive of serous tumor. EUS FNA revealed diagnosis of pseudocyst in these patients which was confirmed on subsequently performed ERCP and resolution after endotherapy. The patients with pre EUS diagnosis of IPMN had EUS features suggestive of pancreatitis with pseudocyst and EUS FNA also revealed diagnosis of pseudocyst.

Conclusions: EUS is an important investigational modality for diagnosis of pancreatic cystic lesions but EUS appearances alone are not helpful in differentiating various causes of cystic lesions and EUS FNA is required for diagnosing pancreatic cystic lesions accurately.

\section{MI21}

\section{Endoscopic ultrasound (EUS) features of mediastinal} tubercular lymphadenopathy

S S Rana, D K Bhasin, $R$ Srinivisan, ${ }^{*} K$ Singh. Department of Gastroenterology and Cytology, ${ }^{*}$ Postgraduate Institute of Medical Education and Research, Sector 12, Chandigarh 160 012, India

Background and Aims: Endoscopic ultrasound (EUS) findings in mediastinal tubercular lymphadenopathy have not been well characterized. This study was done to assess the accuracy of EUS findings of patchy anechoic or hypoechoic areas and hyperechoic foci in mediastinal lymph nodes for the diagnosis of mediastinal tuberculosis.

Patients: Forty-two patients with enlarged mediastinal lymph nodes who underwent EUS guided FNA included. The EUS findings were retrospectively reviewed for size (measured in the long axis), patchy anechoic or hypoechoic areas and hyper echoic foci. The final diagnosis was based on cytological examination of the aspirate as well as the clinical follow up.

Results: The final diagnosis was tuberculosis in 22 patients and remaining 20 patients had non-tubercular diagnosis. Hyperechoic foci in the lymph nodes was seen in 17/22 (77.2\%) of patients with tubercular mediastinal lymphadenopathy whereas it was seen in only $1 / 20(5 \%)$ of patients with non tubercular mediastinal lymphadenopathy $(p=$ $0.00)$. Patchy anechoic or hypoechoic areas were seen in 9/22 (40.9\%) patients with tubercular mediastinal lymphadenopathy whereas none of the patients with nontubercular mediastinal lymphadenopathy had these areas $(p=0.01)$. The patchy anechoic or hypoechoic areas or hyperechoic foci in the mediastinal lymph nodes had accuracy of $69.0 \%$ and $85.7 \%$ respectively for the diagnosis of tuberculosis. 
Conclusion: Patchy anechoic or hypoechoic areas or hyperechoic foci in the mediastinal lymph nodes on EUS are important signs of mediastinal tubercular lymphadenopathy and EUS guided FNA is an useful modality for diagnosis of mediastinal tuberculosis.

\section{MI22}

\section{Clinical profile of eosinophilic gastroenteritis in Kerala}

Charles Panackel, Rajech NA, Benoy Sebastian, Sunil K Mathai. Medical Trust Hospital Kochi

Aim: To study the clinical profile and prevalence of eosinophilic gastroenteritis and eosinophilic colitis among patients attending a tertiary care centre in Kerala.

Methods: A retrospective descriptive analytical study. Duration of study period was from 01/06/09 to 01/07/ 2010. Records of all patients who underwent Endoscopy procedure at the Department were analyzed.

Results: Records of 5100 patients who underwent endoscopic procedure at our institute during the study period were analyzed. Of this 63 patients had a diagnosis of eosinophilic gastroenteritis or colitis. Of this $26(41.26 \%)$ had duodenitis, $11(17.46 \%)$ had ileitis, 16 (25.39\%) had colitis, $4(6.39 \%)$ had eosinophilic gastritis. The male to female ratio was 1.2:1. The age distribution ranged from 10 to 87 years. Most common presentation was abdominal pain in 63\%. Upper GI endoscopic findings included normal study in 11 patients, erosions in stomach and duodenum in 15 patients. Colonoscopy and Ileoscopic findings included normal study in 11 patients, edema and erythema of ileum in 5 patients, aphthous ulcers in 13 patients, Mild colitis in 4 patients.

Discussion: Prevalence of eosinophilic gastroenteritis is on rise. We had 25 cases in preceding year compared to 63 cases during study period. This rise is despite improvement in sanitation and reduction in prevalence of worm infestations. A prospective study is required to analyze the dietary habits, drug history and other risk factors for eosinophilic bowel disorders. These patients have to be followed up for long periods to see how many turns out to be IBD.
Conclusion: Eosinophilic disorders of bowel are on the rise and further prospective studies are required to analyze the cause, response to treatment and long term outcome of these patients.

\section{MI23}

\section{Celiac plexus neurolysis (CPN) for treatment of cancer pain}

A K Satheesh Rao, Prashanth, Sambaiah, Rajkumar Wadhwa. Consultant Gastroenterogists, Vikram Hospital \& Heart Care, Yadavagiri, Mysore

Introduction: Abdominal malignancies produce chronic pain that is often refractory to opioid medications. Despite their relative efficacy for pain relief, opioids have adverse effects such as drowsiness, delirium, dry mouth, anorexia, constipation, nausea and vomiting. Therefore, invasive methods such as celiac plexus neurolysis (CPN) have been used to alleviate the pain and reduce the risk of druginduced adverse effects. We are presenting our experience in CPN in a single centre tertiary care hospital. In the last one year we performed CPN for five patients to control pain with different intra abdominal malignancies. Two patients had pancreatic cancer, one with hepatocellular cancer, one with cholangio carcinoma and one with cancer of the stomach.

Results: CPN was performed using the linear array echoendoscope. Pain relief was reported in four out of five patients and two of them termed the pain control as 'excellent' during the first 2 weeks. Follow up beyond 3 months suggested persistent benefit. Partial or complete pain relief continued in all patients, alive at 3 months post CPN and 70-90\% until death. Common adverse affects noticed are abdominal bloating [90\%], and hypotension [20\%].

Discussion: EUS-CPN is an easy to perform and relatively safe procedure for the palliation of cancer-related pain Adverse effects are common, but transient and mild. Further trials are necessary to compare $\mathrm{CPN}$ to other pain relief treatments, such as systemic or spinal delivery of opioids. 